Old Dominion University

ODU Digital Commons

Fall 2013

\title{
Measurement of the Spin Structure Function GD1 of the Deuteron and Its Moments at Low Q2
}

Krishna P. Adhikari

Old Dominion University

Follow this and additional works at: https://digitalcommons.odu.edu/physics_etds

Part of the Nuclear Commons

\section{Recommended Citation}

Adhikari, Krishna P.. "Measurement of the Spin Structure Function GD1 of the Deuteron and Its Moments at Low Q2" (2013). Doctor of Philosophy (PhD), Dissertation, Physics, Old Dominion University, DOI:

$10.25777 /$ ekwq-m362

https://digitalcommons.odu.edu/physics_etds/27

This Dissertation is brought to you for free and open access by the Physics at ODU Digital Commons. It has been accepted for inclusion in Physics Theses \& Dissertations by an authorized administrator of ODU Digital Commons. For more information, please contact digitalcommons@odu.edu. 


\title{
MEASUREMENT OF THE SPIN STRUCTURE FUNCTION $G_{1}^{D}$ OF THE DEUTERON AND ITS MOMENTS AT LOW $Q^{2}$
}

\author{
by \\ Krishna P. Adhikari \\ B.Sc. 1998, Tribhuvan University \\ M.Sc. 2001, Tribhuvan University \\ M.S. June 2006, Old Dominion University
}

\author{
A Dissertation Submitted to the Faculty of \\ Old Dominion University in Partial Fulfillment of the \\ Requirement for the Degree of \\ DOCTOR OF PHILOSOPHY \\ PHYSICS \\ OLD DOMINION UNIVERSITY \\ Nov 2013
}

Approved by:

Sebastian E. Kuhn (Director)

T. Wallace Van Orden

Gail E. Dodge

Leposava Vuskovic

John A. Adam 


\title{
ABSTRACT \\ MEASUREMENT OF THE SPIN STRUCTURE FUNCTION $G_{1}^{D}$ OF THE DEUTERON AND ITS MOMENTS AT LOW $Q^{2}$
}

\author{
Krishna P. Adhikari \\ Old Dominion University, 2013 \\ Director: Dr. Sebastian E. Kuhn
}

Double polarization cross section differences $\left(\Delta \sigma_{\|}\right)$for proton and deuteron targets have been measured in the EG4 experiment using the CLAS detector at Jefferson Lab. Longitudinally polarized electron beams at relatively low energies of 1.056, 1.337, 1.989, 2.256 and $3.0 \mathrm{GeV}$ from the CEBAF accelerator were scattered off longitudinally polarized $\mathrm{NH}_{3}$ and $\mathrm{ND}_{3}$ targets. Scattered electrons were recorded at very low scattering angles (down to $\theta=6^{\circ}$ ) with the help of a new dedicated Cherenkov counter and a special magnetic field setting of the CLAS detector in order to measure the cross section differences in the resonance region ( $1.08 \mathrm{GeV}<W<2.0 \mathrm{GeV})$ at very low momentum transfers $\left(Q^{2}\right.$ for the deuteron was as low as $0.02 \mathrm{GeV}^{2}$ ). These measurements on the deuteron were used to extract the deuteron's spin structure function $g_{1}$ as well as the product $A_{1} F_{1}$ of the virtual photon asymmetry $A_{1}$ and the unpolarized structure function $F_{1}$. These extracted quantities, in turn, were used to evaluate three important integrals for the deuteron - the first moment $\left(\Gamma_{1}\right)$ of $g_{1}$, the extended Gerasimov-Drell-Hearn (GDH) integral $\left(\bar{I}_{T T}\right)$, and the generalized forward spin polarizability $\left(\gamma_{0}\right)$. These measurements extend and improve the world deuteron data on $g_{1}$ to the previously unmeasured low $Q^{2}$ region. The data, in combination with the corresponding proton data from the same experiment, will be valuable to extract $g_{1}$ on the neutron in the same kinematics. They will shed more light on the nucleon spin structure in the region of quark-confinement as well in the transition region between hadronic and partonic degrees of freedom. In addition, the three integrals evaluated from the measured data are compared to predictions from different Chiral Perturbation Theory $(\chi \mathrm{PT})$ calculations and phenomenological models. Extrapolations of the integrals (especially the GDH sum and the polarizability) to the real photon point $\left(Q^{2}=0\right)$ enable us to test the validity of the predictions for their real photon counterparts. The new results have extended and improved the very low $Q^{2}$ data on $g_{1}$ and the corresponding results on moments compare very well with the latest $\chi \mathrm{PT}$ and phenomenological calculations (especially near the photon point). 
Copyright, 2014, by Krishna P. Adhikari, All Rights Reserved. 


\section{ACKNOWLEDGMENTS}

This dissertation could not have come to fruition without the advice, help and support of many people.

First and foremost, I would like to express my deepest gratitude to my advisor Dr. Sebastian E. Kuhn for giving me the opportunity to work with him, providing constant guidance, sage advice, and inspiration throughout the years spent doing this work. He had faith in me, patience with me and was eager to help and support me in every possible way. I will always be indebted to him for the huge amount of time and energy he invested in me and for the knowledge and wisdom he shared.

My wife Anita and my parents Ishwori and Buddhi Maya deserve no less credit for having the patience with me and constantly providing me with the love, motivation and support. I will remain equally indebted to them for my entire life. I also want to thank my sister Laxmi, and brothers Bharat and Bishnu for their love and support.

I would like to thank Professors Gail E. Dodge, J. Wallace Van Orden, Leposava Vuskovic and John Adam for kindly serving on my thesis committee and providing me helpful suggestions and support.

I am especially grateful to Dr. Gail E. Dodge for her words of encouragements, support and research guidance from the very first days of my work on the project as well as from her past capacity as the Chair of the Physics Department.

I also want to express my special gratitude to Dr. J. Wallace Van Orden who gave me an opportunity to work with him for more than a year, for serving on my thesis committee and for teaching me courses on Classical and Quantum Mechanics.

I would also like to express my special gratitude to my Graduate Program Director Dr. Leposava Vuskovic for her ever willingness and readiness to help on administrative, academic and personal matters. I am particularly thankful to her for agreeing to be my replacement advisor for the doctoral hooding ceremony.

I am very thankful to Dr. Lawrence Weinstein for his support and words of wisdom from the first days since I joined ODU. He has been inspirational in many ways and has been of great help on computational ideas, and physics concepts whenever I have needed and asked for.

I am greatly indebted to Dr. Rocco Schiavilla, Dr. Ian Balitsky, Dr. Van Orden, Dr. Anatoly Radyuskin, and Dr. Maskov Amarian Amarian who taught me various graduate 
courses offered at ODU, helping build a strong foundation on the subjects covered by the courses. The skills and tools that I acquired by attending their classes prepared me and helped me take on and complete this work.

I am also very thankful to Dr. Stephen Bueltmann for always being encouraging and helpful. Thank you Tom Hartlove for your support and ever willingness to help. Thank you Walt Hooks, Annette Guzman-Smith, Delicia Malin, Lisa Okun, and Justin Mason for making me feel home with all kinds of support. And, I will never forget the kindness and the support I got from the late Robert E. Evans.

My special thanks are also due to the EG4 spokespersons - Drs. Alexander Deur, Marko Ripani and Karl Slifer - who has been of tremendous help and encouragement over the whole period of time ever since I joined this project. Other EG4 collaborators especially Dr. Raffaella De Vita, Hyekoo Kang, Sarah Phillips, Vadim Drozdov, Dr. Peter Bosted, Dr. Xiaochao Zheng - have also been of great help whenever I needed. I want to express a heartfelt gratitude to each of them.

My special thanks is also extended to Dr. Volker Burkert for supporting me by being my Jlab sponsor.

I am also greatly indebted to Jixie Zhang and Mike Mayer on their frequent helps especially on computational and data analysis issues in addition to their many other friendly helps and advices on several things. I also feel indebted to the helps I got from Nevzat Guler, Chandra Nepali, and Megh Niroula.

Finally, I would like to thank all the friends especially Suman Koirala, Gambhir Ranjit, Rajendra Adhikari, Arpan Subedi, Binod Dhakal, Sundar Dhakal, Krishna H. Bhatta, Bishnu Parajuli, Kishor Khanal, Bishnu Gyawali, Surya Mishra, Krishna H. Poudel, Bill Ford, Kurnia Foe, Sharon Carecia, Donika Plyku, Ivan Koralt, Mike Kunkel, Eman Ahmed, Asma Begum, Mustafa Canan, Marija Raskovic, Giovanni Chirilli, Jan Drake, Serkan Golge, Anup Khanal, Sushil Poudel, Suman Neupane, Vinit Jha, Manoj Rajaure, Sanjaya Shrestha, Pranjal Kalita, Rocky Shrestha, Ajaya Dhakal, Nikhil KChetry, Janesh Thapa, S H Sathis Indika for their helps and friendships that made my life at and around ODU an enjoyable experience. I cannot express my gratitude enough to all of those named here as well as others who have directly and indirectly touched and positively affected my life. Last but not the least, a special thanks to Gabriel Franke for reviewing this dissertation. 


\section{TABLE OF CONTENTS}

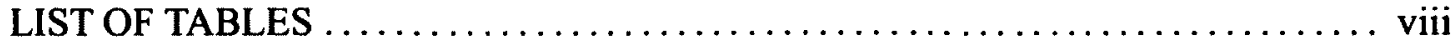

LIST OF FIGURES $\ldots \ldots \ldots \ldots \ldots \ldots \ldots \ldots \ldots \ldots \ldots \ldots \ldots \ldots \ldots \ldots \ldots \ldots \ldots \ldots \ldots \ldots \ldots$

Chapter

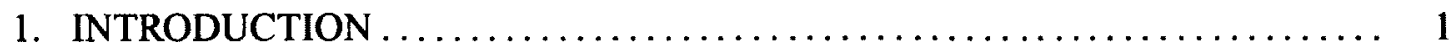

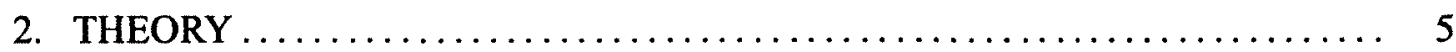

2.1 INCLUSIVE ELECTRON SCATTERING $\ldots \ldots \ldots \ldots \ldots \ldots \ldots \ldots$

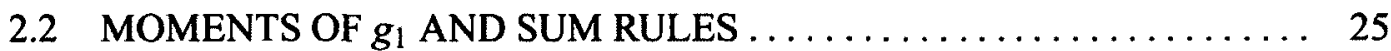

2.3 THEORETICAL TOOLS ......................... 33

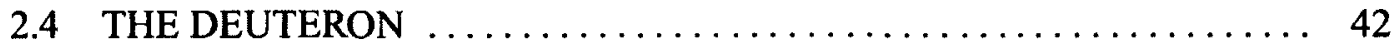

3. EXPERIMENTAL TOOLS AND SET UP..................... 47

3.1 CEBAF LINEAR ACCELERATOR $\ldots \ldots \ldots \ldots \ldots \ldots \ldots \ldots \ldots \ldots \ldots \ldots$

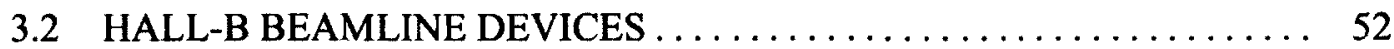

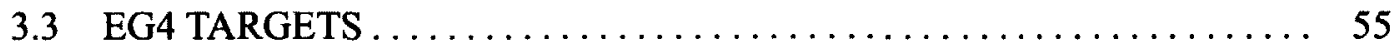

3.4 CEBAF LARGE ACCEPTANCE SPECTROMETER (CLAS) . . . . . . . 59

3.5 TRIGGER AND DATA ACQUISITION SYSTEM (DAQ) . . . . . . . . 69

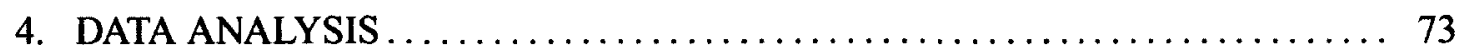

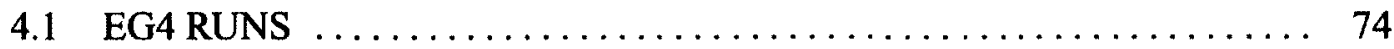

4.2 RAW DATA PROCESSING - CALIBRATION AND RECONSTRUCTION 74

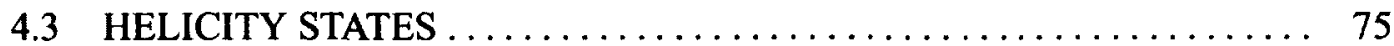

4.4 DATA QUALITY AND STABILITY CHECKS $\ldots \ldots \ldots \ldots \ldots \ldots \ldots \ldots$

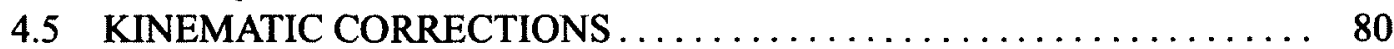

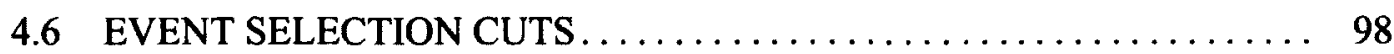

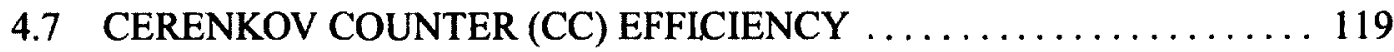

4.8 PION CONTAMINATION CORRECTIONS $\ldots \ldots \ldots \ldots \ldots \ldots \ldots \ldots . \ldots \ldots$

$4.9 e^{+} e^{-}$-PAIR SYMMETRIC CONTAMINATION CORRECTIONS $\ldots \ldots \ldots 127$

4.10 STUDY OF $\mathrm{NH}_{3}$ CONTAMINATION OF EG4 ND 3 TARGET . . . . . . 128

4.11 SIMULATION AND APPROACH TO ANALYSIS $\ldots \ldots \ldots \ldots \ldots \ldots \ldots$

4.12 RADIATIVE CORRECTIONS ........................ 137

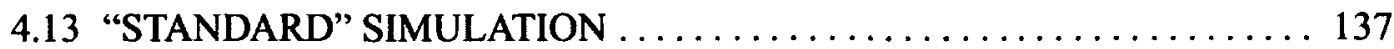

4.14 COMPARISON OF DATA AND SIMULATION $\ldots \ldots \ldots \ldots \ldots \ldots \ldots \ldots$

4.15 METHOD TO EXTRACT $g_{1}$ AND $A_{1} F_{1} \ldots \ldots \ldots \ldots \ldots \ldots \ldots \ldots \ldots \ldots$

4.16 SYSTEMATIC ERROR ESTIMATIONS IN $g_{1}$ AND $A_{1} F_{1} \ldots \ldots \ldots \ldots$

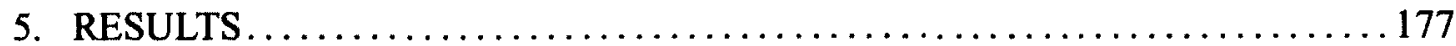


5.1 THE EXTRACTED VALUES OF $g_{1}$ AND $A_{1} F_{1} \ldots \ldots \ldots \ldots \ldots \ldots$

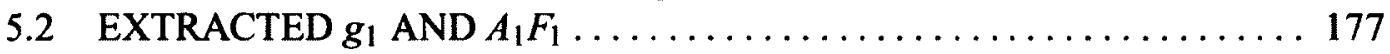

5.3 MOMENTS OF DEUTERON SPIN STRUCTURE FUNCTIONS $\ldots \ldots \ldots$

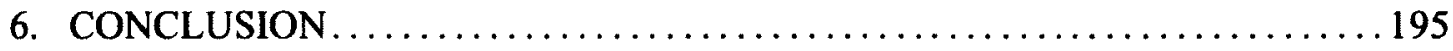
APPENDICES

A. DERIVATION OF THE GDH SUM RULE $\ldots \ldots \ldots \ldots \ldots \ldots \ldots \ldots \ldots$

B. FFREAD CARDS USED BY GSIM $\ldots \ldots \ldots \ldots \ldots \ldots \ldots \ldots \ldots \ldots \ldots$

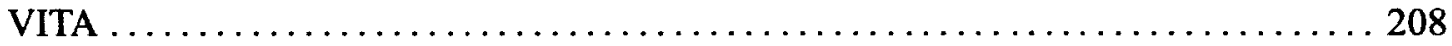




\section{LIST OF TABLES}

Table

Page

1. DC-smearing scales determined for different beam energies. . . . . . . 148

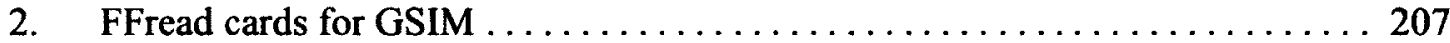




\section{LIST OF FIGURES}

$\begin{array}{lll}\text { Figure Page } & \text { Pag }\end{array}$

1. Inclusive scattering (Born Approximation) $\ldots \ldots \ldots \ldots \ldots \ldots \ldots \ldots \ldots \ldots$

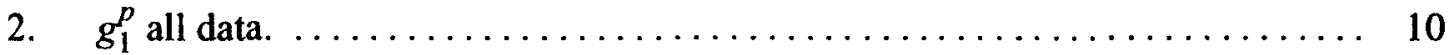

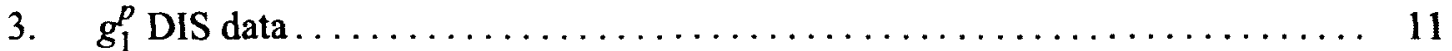

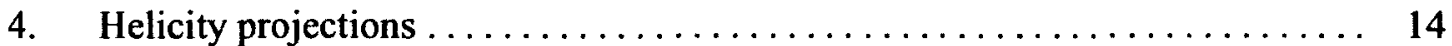

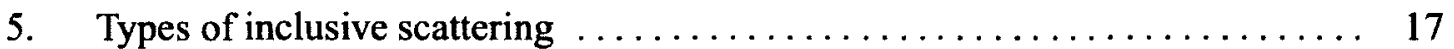

6. $\quad F_{2}^{p}$ showing the scaling behaviour in DIS $\ldots \ldots \ldots \ldots \ldots \ldots \ldots \ldots \ldots \ldots \ldots \ldots \ldots \ldots$

7. Predictions for $\Gamma_{1}^{d}$ and some data $\ldots \ldots \ldots \ldots \ldots \ldots \ldots \ldots \ldots \ldots \ldots \ldots \ldots \ldots$

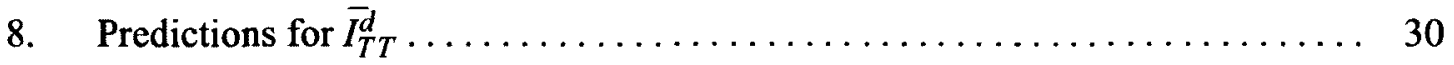

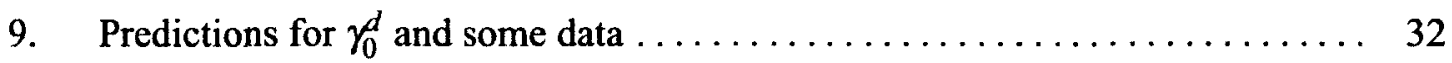

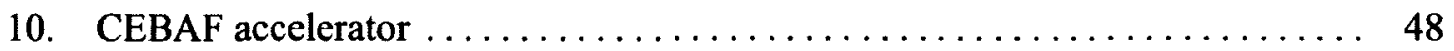

11. CEBAF accelerator (components) $\ldots \ldots \ldots \ldots \ldots \ldots \ldots \ldots \ldots \ldots \ldots \ldots$

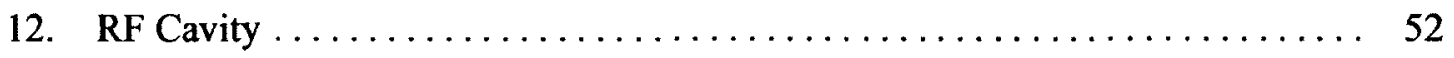

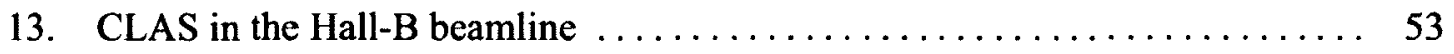

14. Moeller Polarimeter. ....................... 54

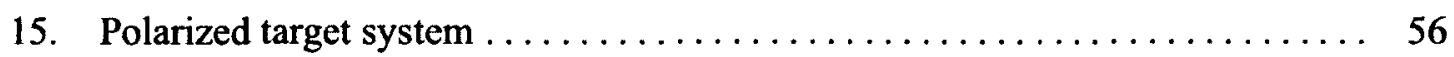

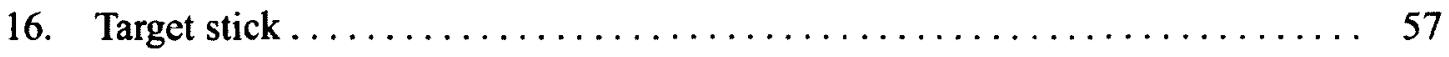

17. Electron energy levels $\ldots \ldots \ldots \ldots \ldots \ldots \ldots \ldots \ldots \ldots \ldots \ldots \ldots \ldots \ldots \ldots$

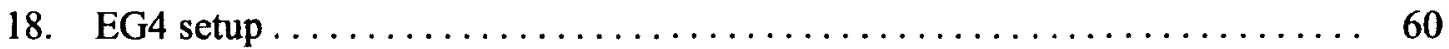

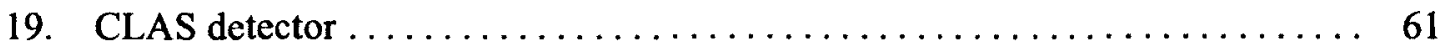

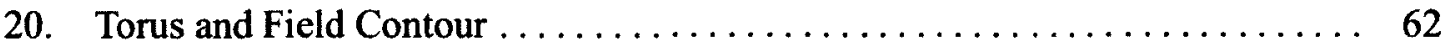

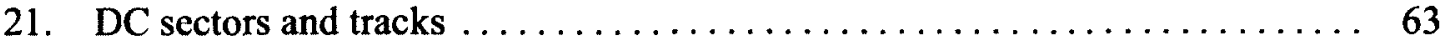

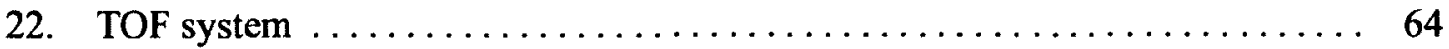


23. The Standard CLAS Cherenkov Counter $\ldots \ldots \ldots \ldots \ldots \ldots \ldots \ldots \ldots \ldots$

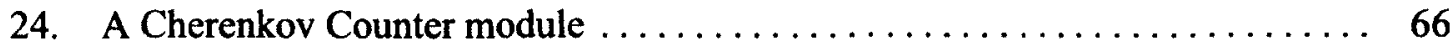

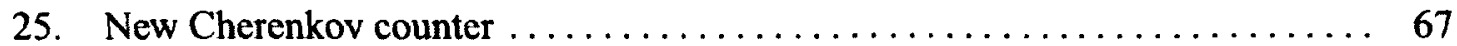

26. A new CC segment and light reflections $\ldots \ldots \ldots \ldots \ldots \ldots \ldots \ldots \ldots$

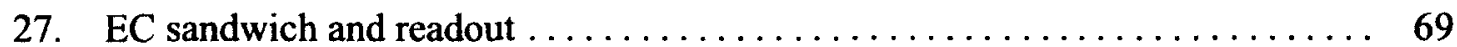

28. CLAS DAQ data flow schematic $\ldots \ldots \ldots \ldots \ldots \ldots \ldots \ldots \ldots \ldots \ldots \ldots \ldots \ldots \ldots$

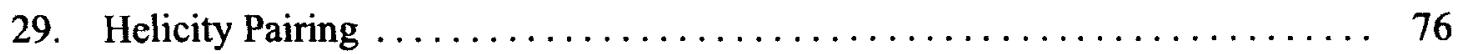

30. Normalized total yield $\left(2.0 \mathrm{GeV}\left(\mathrm{ND}_{3}\right)\right) \ldots \ldots \ldots \ldots \ldots \ldots \ldots \ldots \ldots \ldots \ldots \ldots$

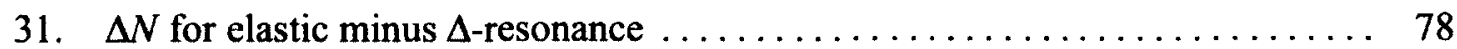

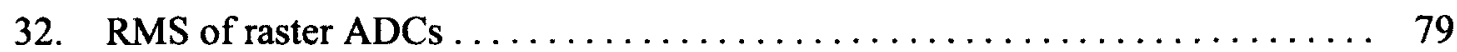

33. Raster correction geometry illustration (Figure courtesy of S. Kuhn) ...... 81

34. Raster correction geometry illustration (Figure courtesy - S. Kuhn) ....... 82

35. Beam coordinates $\mathrm{x}$ and $\mathrm{y}$ calculated with the raster correction procedure. ... 83

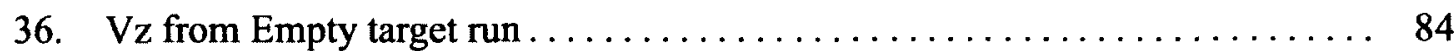

37. An exaggerated figure showing the effect of multiple scattering. . . . . . . 89

38. Background removal from $\Delta E \ldots \ldots \ldots \ldots \ldots \ldots \ldots \ldots \ldots \ldots \ldots \ldots \ldots$

39. Effects of corrections ep-elastic events $\ldots \ldots \ldots \ldots \ldots \ldots \ldots \ldots \ldots \ldots$

40. Effects of corrections on $p\left(e, e^{\prime} \pi^{+} \pi^{-}\right) X$ events $\ldots \ldots \ldots \ldots \ldots \ldots \ldots \ldots$

41. Missing mass minus neutron mass ...................... 97

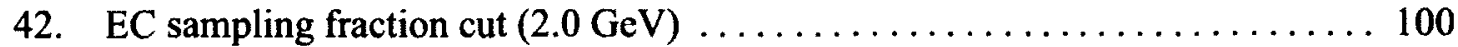

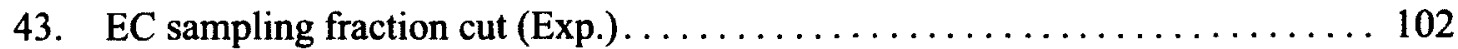

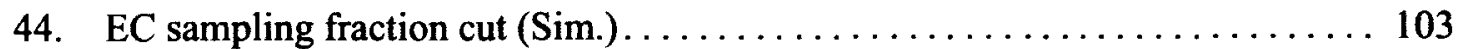

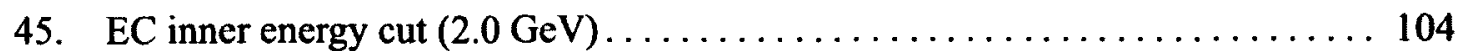

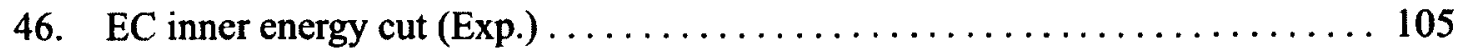




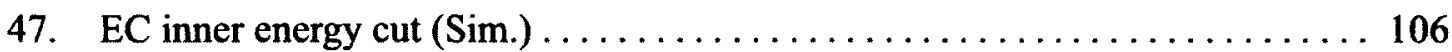

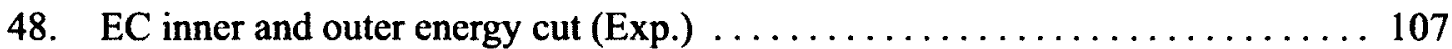

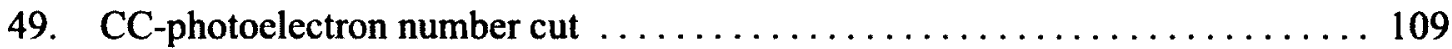

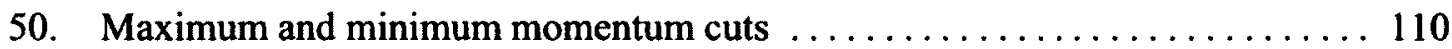

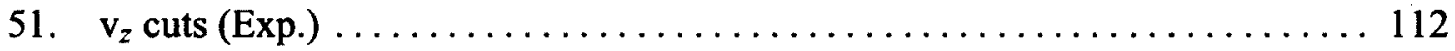

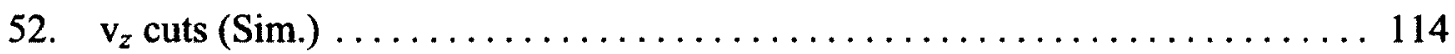

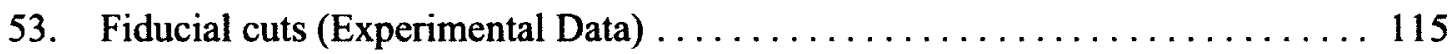

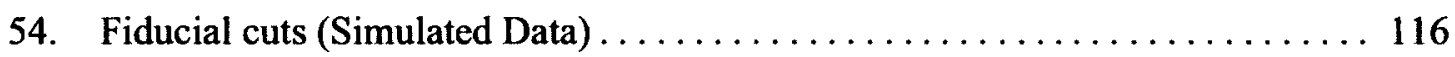

55. Regular/EC-only plots $\ldots \ldots \ldots \ldots \ldots \ldots \ldots \ldots \ldots \ldots \ldots \ldots \ldots \ldots \ldots \ldots \ldots$

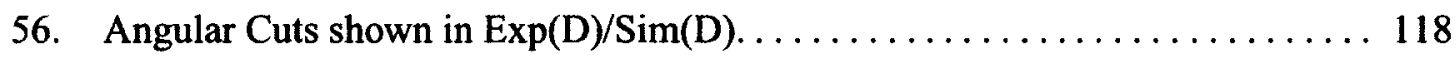

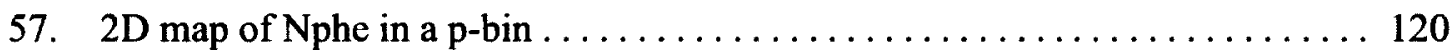

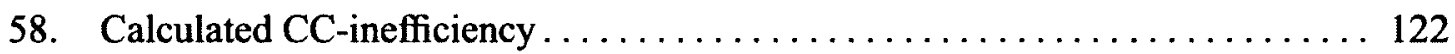

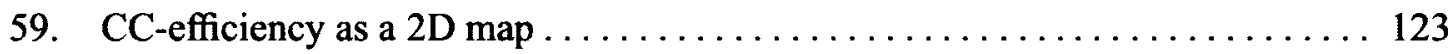

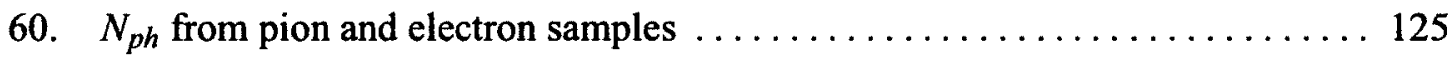

61. Calculation of pion contamination and fits. $\ldots \ldots \ldots \ldots \ldots \ldots \ldots \ldots \ldots$

62. Fits of pair-symmetric contamination $\ldots \ldots \ldots \ldots \ldots \ldots \ldots \ldots \ldots \ldots \ldots \ldots \ldots \ldots$

63. $\Delta=p_{p} \cdot\left(\sin \theta_{q}-\sin \theta_{p}\right)$ for quasi-elastic events $\ldots \ldots \ldots \ldots \ldots \ldots \ldots \ldots \ldots \ldots \ldots \ldots$

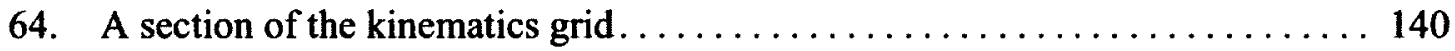

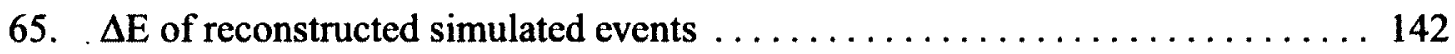

66. DC-smearing effects on elastic peak $\ldots \ldots \ldots \ldots \ldots \ldots \ldots \ldots \ldots \ldots \ldots \ldots \ldots \ldots$

67. Background subtraction to get elastic peak .................. 144

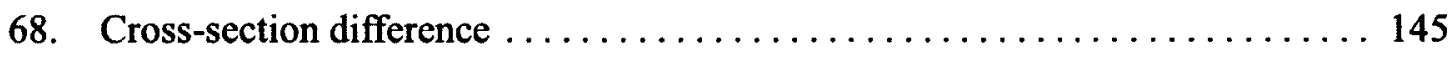

69. $E_{\text {beam }}$ dependence of DC smearing (Experimental) $\ldots \ldots \ldots \ldots \ldots \ldots \ldots$

70. $E_{\text {beam }}$ dependence of DC smearing (Experimental) $\ldots \ldots \ldots \ldots \ldots \ldots \ldots$ 
71. $\Delta n$ for data and simulation $\ldots \ldots \ldots \ldots \ldots \ldots \ldots \ldots \ldots \ldots \ldots \ldots \ldots \ldots, \ldots \ldots \ldots$

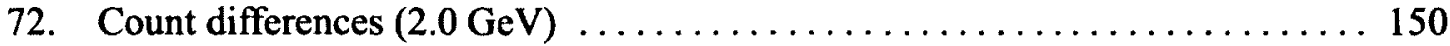

73. Data to simulation ratios vs $Q^{2} \ldots \ldots \ldots \ldots \ldots \ldots \ldots \ldots \ldots \ldots \ldots \ldots \ldots \ldots \ldots, 152$

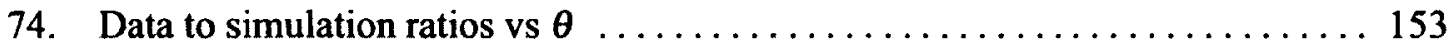

75. Data to simulation ratios vs $Q^{2}(2.0 \mathrm{GeV}) \ldots \ldots \ldots \ldots \ldots \ldots \ldots \ldots \ldots \ldots \ldots \ldots \ldots$

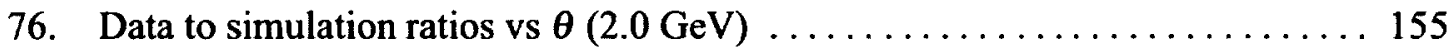

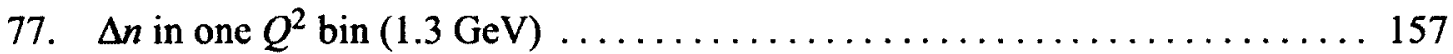

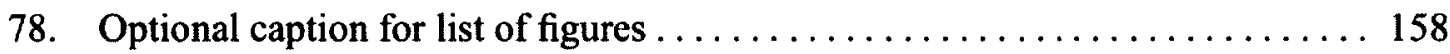

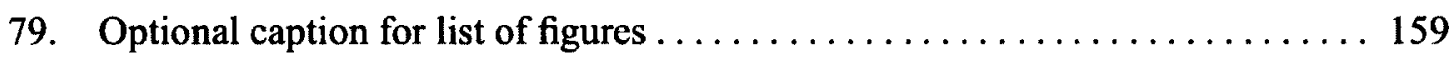

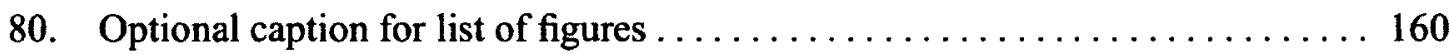

81. Breakdown of systematic errors on $g_{1}$ for $1.3 \mathrm{GeV} \ldots \ldots \ldots \ldots \ldots \ldots \ldots$

82. Breakdown of systematic errors on $g_{1}$ for $1.3 \mathrm{GeV} \ldots \ldots \ldots \ldots \ldots \ldots . \ldots 169$

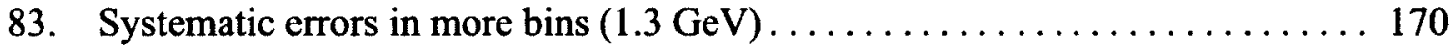

84. Breakdown of systematic errors on $g_{1}$ for $2.0 \mathrm{GeV} \ldots \ldots \ldots \ldots \ldots \ldots . \ldots 171$

85. Systematic errors in more bins $(2.0 \mathrm{GeV}) \ldots \ldots \ldots \ldots \ldots \ldots \ldots \ldots \ldots \ldots \ldots \ldots \ldots \ldots$

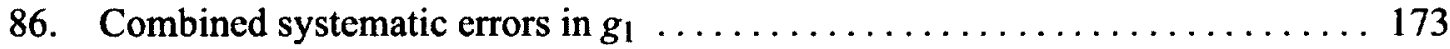

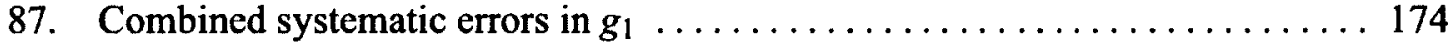

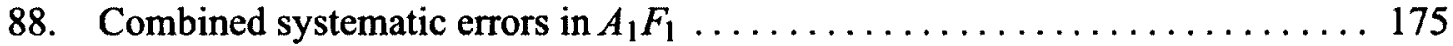

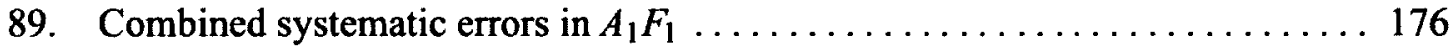

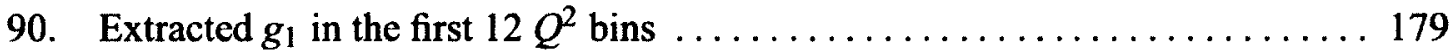

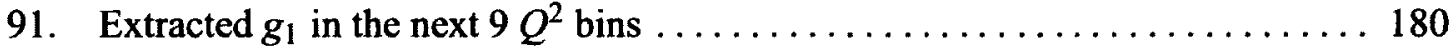

92. Extracted $A_{1} F_{1}$ in the first $12 Q^{2}$ bins $\ldots \ldots \ldots \ldots \ldots \ldots \ldots \ldots \ldots \ldots \ldots \ldots \ldots \ldots \ldots \ldots$

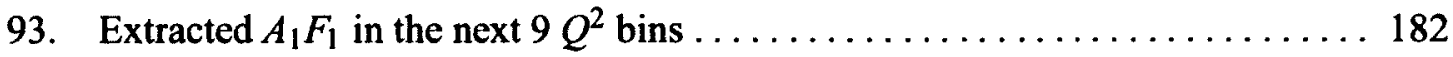

94. Combined $g_{1}$ (in first $12 Q^{2}$ bins) $\ldots \ldots \ldots \ldots \ldots \ldots \ldots \ldots \ldots \ldots \ldots \ldots \ldots \ldots \ldots \ldots \ldots$ 


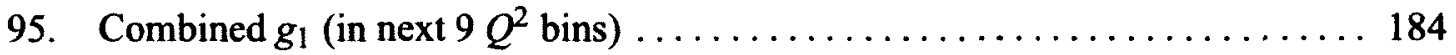

96. Combined $A_{1} F_{1}$ (in first $12 Q^{2}$ bins) $\ldots \ldots \ldots \ldots \ldots \ldots \ldots \ldots \ldots \ldots \ldots \ldots \ldots \ldots \ldots \ldots \ldots \ldots \ldots \ldots$

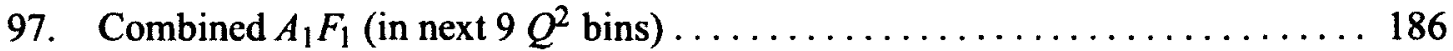

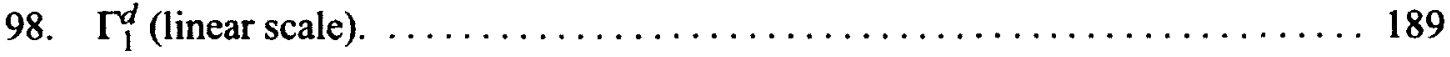

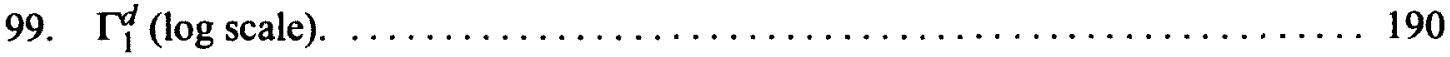

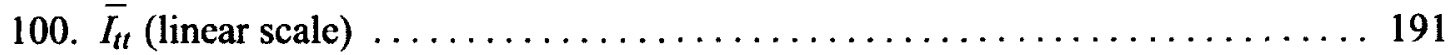

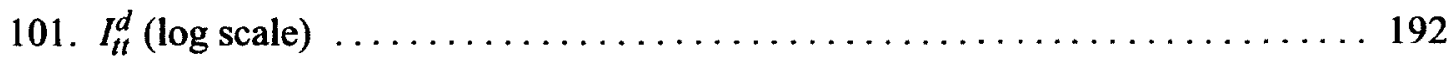

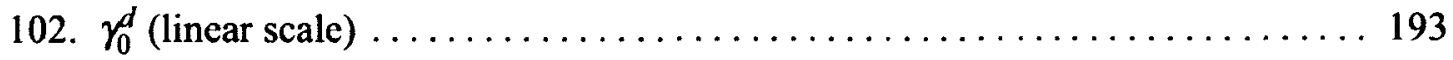

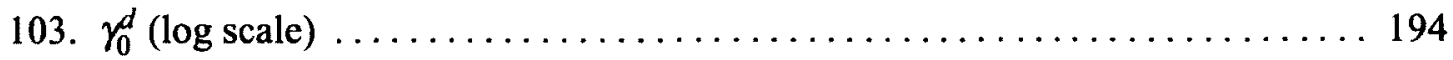




\section{CHAPTER 1}

\section{INTRODUCTION}

The goal of the natural sciences as a whole is to understand the natural world - to understand its structure and the underlying principles as much as possible. From centuries of experimental and theoretical scientific effort, we have come to know a lot about nature, and we have already been exploiting those scientific achievements whenever and wherever we find them useful. From our own field of physics, for example, we know a lot about the properties of bulk matter, about atomic structure and about the even lower sub-strata of the world, i.e. the sub-microscopic world of nuclei, nucleons and many other sub-nuclear particles. In spite of achieving an unprecedented level of understanding, there are still a lot of questions that remain unanswered. One such subject that has drawn a great deal of attention from the nuclear and particle physics community is the structure of the nucleons (i.e., protons and neutrons) and their intrinsic property called "spin".

According to modern physics, spin is an intrinsic form of angular momentum ${ }^{1}$ [1] carried by elementary particles (electrons, quarks, photons) as well as composite particles (hadrons, atomic nuclei, atoms as a whole, molecules etc) [2]. The concept of spin as an intrinsic property of a particle was introduced by Uhlenbeck and Goudsmit in order to explain the results of the Stern and Gerlach experiment [3] as well as other puzzling observations from the early 1920 s such as the hyperfine splitting in atomic spectral lines. Later, in 1933, Stern also measured the proton's much smaller (relative to the electron's) magnetic moment using his improved apparatus [4] and found that the measured value did not agree with the value predicted by Dirac theory for particles with spin-1/2 and no structure (i.e., point particles) ${ }^{2}$. This discovery of the anomalous magnetic moment was the first concrete signature for the fact that the proton is not a simple point particle like an electron, but rather had a substructure, thus heralding a new still ongoing era in the

\footnotetext{
${ }^{1}$ Classically, angular momentum is a vector quantity that represents the amount of rotation an object has, taking into account its mass and shape.

${ }^{2}$ Dirac's prediction for a point like particle of charge q, mass $\mathrm{M}$ and spin $\mathrm{S}$ is $\mu_{D}=q \hbar S / M$, but the measurements showed that $\mu_{p}=2.79 \mu_{N}$ and $\mu_{n}=-1.91 \mu_{N}$, where $\mu_{N}=e h / 2 M_{p}=3.1525 \times 10^{-14} \mathrm{MeV} / T=$ $5.05078324(13)^{-27} J . T^{-1}$ is the Nuclear magneton.
} 
quest to understand the nucleon's structure and the origin of its spin. Many decades later, experiments at powerful accelerators have provided more independent confirmations of the nucleon substructure.

A truly vast amount of data on the inelastic structure of the nucleons has been accumulated since the late 1960s from both fixed target and colliding beam experiments with polarized as well as un-polarized incident photons, (anti)electrons, muons and (anti)neutrinos as well as (anti)protons on a variety of targets (both polarized and unpolarized) from hydrogen through iron [5]. The initial measurements at SLAC confirmed the picture of the nucleon as made up of partons (now identified with quarks and gluons). Since then more precise measurements have been conducted at several accelerators, improving our knowledge and understanding about the nucleon structure (both spin-dependent and spin-averaged), and, at the same time, continuing to give us new and sometimes very surprising results such as the original "European Muon Collaboration (EMC)-Effect" [6], the violation of the Gottfried sum rule [7, 8], and the so-called "Spin-Crisis" [9, 10] (see below).

With such a vast amount of experimental data available, a lot is now known about the spin-averaged quark structure of the nucleon, but a lot less is known about the spinstructure of the nucleon in terms of its constituents quarks and gluons [5]. In a simple non-relativistic model one would expect the quarks to carry the entire spin of the nucleon, but one of the early more realistic theories that explained the partonic substructure of the nucleon, the Naive Parton Model (NPM), predicted that $60 \%$ of the nucleon spin is carried by the quarks [11].

The polarized beam and target technologies have greatly advanced during the last three decades, and many subsequent experiments on nucleons and some nuclei have contributed to the extraction of their spin structure functions $g_{1}$ and $g_{2}$, which carry information on how the spin is distributed inside the target. One of the first experiments carried out at SLAC, in a limited kinematic region, seemed to confirm the predictions of the NPM. However, a subsequent, more precise measurements over a larger kinematic region performed by the EMC experiment at CERN reported that, contrary to the NPM predictions, only $12 \pm 17 \%$ (i.e., practically none) of the spin is carried by the quarks $[9,10]$. This discovery of the so-called "spin crisis" sparked a large interest in measuring the spin content of the nucleon, giving birth to several experiments (completed, underway and proposed) around the globe. The theoretical developments of Quantum Chromodynamcis (QCD) - the quantum field theory that describes the nuclear interaction between the quarks and gluons - have clarified our picture of the nucleon spin structure in great detail. With the discovery of a unique 
QCD property known as "asymptotic freedom", quarks are known to be essentially free and interact very weakly at higher energies (or shorter distances) allowing perturbative QCD (PQCD) calculations of testable predictions for processes involving high energy or high momentum transfers [12]. The so-called Bjorken sum rule, which relates results from inclusive, polarized deep inelastic scattering (DIS) (a high energy process) to the fundamental axial coupling constant $\left(g_{A}\right)$ of neutron beta decay, is a precise test of QCD. The interpretation of existing DIS results has verified the Bjorken sum rule at the level of $10 \%$ accuracy and has shown that only about $30 \pm 10 \%$ of the nucleon spin is carried by the quarks; the rest of the spin must reside either in gluons or orbital angular momentum of its constituents. Experiments to measure the gluon contribution are underway at Brookhaven National Laboratory (BNL) and CERN.

Probing nucleon structure on the other end of the energy scale (i.e. probing with low momentum transfers) provides information about the long distance structure of the target, which is also associated with static properties of the nucleon. In this low energy regime, however, QCD calculations with the established perturbative methods become difficult or even impossible because the coupling constant $\left(\alpha_{s}\right)$ becomes very large, and so the perturbative expansions (in powers of $\alpha_{s}$ ) do not converge. In other words, in this energy regime, the partons become very strongly coupled to the point of being confined into hadrons which now emerge as the new (effective) degrees of freedom for the interaction. Therefore, other methods must be relied on to make predictions in these non-perturbative energy scales. For example, at very low energies, effective theories such as chiral perturbation theory $(\chi \mathrm{PT})$ are used. There is also an intermediate region where neither of these approaches (PQCD or $\chi \mathrm{PT}$ ) is expected to work. In this region, it is expected that lattice QCD methods will provide testable predictions in the near future. There are also some phenomenological models aimed at describing the entire kinematic range. The description of the low energy regime in terms of these theories and models is still a challenge and theories used here are still fraught with several issues (see Chap. 2). There are already several predictions (for both nucleons as well as some light nuclei such as the deuteron and Helium-3) from these low energy theories and models on various observables which can tested using experimental data. Therefore, having high precision data at the lowest possible momentum transfer is very important to test these already available predictions. In addition, new results will also help constrain future calculations and provide input for detailed corrections to higher energy data. 
With that perspective and motivation, the "EG4" experiment (E06-017) for a precision double polarization measurement at low momentum transfer using both proton and deuteron targets and the Hall B CLAS detector was performed at Jefferson Lab. In addition to the usefulness of the measured deuteron data for testing theoretical predictions calculated for the deuteron itself, the data are also useful for extracting neutron data ${ }^{3}$ in combination with similar data from the proton target. An experiment with the similar goal of probing the neutron at low momentum transfers but using ${ }^{3} \mathrm{He}$ was performed in Hall A [13]. However, due to the not-fully-understood complexities of nuclear medium effects, neutron information extracted from only one type of nuclear target cannot give us enough confidence in our measurements. So, having results from different types of targets is very important for better confidence in the extracted neutron results, which will enable us to test the theoretical and model predictions as is done for other targets (deuteron, proton). The data on the deuteron (and eventually on the neutron) will not only be useful to test the theoretical predictions at low but finite momentum transfers but they can also be used to extrapolate to the real photon absorption limit, thus providing tests of some long standing predictions such as the Gerasimov-Drell-Hearn (GDH) sum rule (derived not from the aforementioned low energy effective theories but independently from general principles). The analysis of the deuteron data is the subject of this thesis and the proton target data collected by EG4 are being analyzed by another member of the collaboration.

In the future, we will extract information from the deuteron and proton data from the EG4 experiment to provide a self-consistent determination of the Bjorken sum, helping us to understand the transition from the partonic to hadronic descriptions of the strong interaction. The data will also be useful in studying the validity of quark-hadron duality in the spin sector, thus helping further to understand the transition from the partonic to hadronic pictures.

In this thesis, I will describe the work done to analyze the deuteron data from the EG4 experiment and will present and describe the preliminary results obtained for the deuteron target. For that purpose, I will first describe the theoretical formalism in Chapter 2. Then, in the third chapter, the experimental details are discussed. After that, the details of the data analysis are described in Chapter 4 . The preliminary results calculated for the deuteron are presented in Chapter 5. Finally, Chapter 6 presents a summary and conclusions.

\footnotetext{
${ }^{3}$ Due to the relatively very short lifetime and various other complexities, no free neutron target has been devised yet. All the relevant neutron information so far has come from measurements on nuclear targets (mostly very light nuclei such as ${ }^{2} \mathrm{H}$ and ${ }^{3} \mathrm{He}$ ).
} 


\section{CHAPTER 2}

\section{THEORY}

\subsection{INCLUSIVE ELECTRON SCATTERING}

High energy particle scattering processes provide very powerful microscopes to examine objects such as nuclei and nucleons. Scattering of leptons (most commonly electrons) is one of the most extensively used processes. For example, the scattering of high energy leptons off nucleons has played a key role in determining the partonic structure of the nucleons. Following are some of the advantages of lepton (and in particular electron) scattering:

- Leptons interact through the electroweak interaction which is very well understood.

- The interaction is relatively weak, thus enabling measurements with only small disturbances to the target structure.

- In electron scattering, one can, moreover, control and vary the polarization of the virtual photon (exchanged during the interaction) by changing the electron kinematics. This allows the separation of the charge and current interaction. Data from the scattering of polarized electrons by polarized targets allows one to examine the target's strong-interaction spin structure.

- A great advantage of electrons is that they can be copiously produced in the laboratory relatively easily and at low costs, and since they are charged, they can readily be accelerated and detected. (It is not as easy and cheap to produce and handle the other lepton types.

In this section, we discuss the process of inclusive electron scattering (in which only the scattered electron is detected ignoring the rest of the components of the final state after the interaction). In doing so, the relevant kinematic variables and related physical quantities to be measured or calculated from the process will be introduced and some of their relations with one another will be deduced and discussed. 


\subsubsection{KINEMATIC VARIABLES}

A lepton scattering process, in which an incoming lepton represented by $l(p)$ of four momentum $p=p^{\mu}=(E, \vec{k})$ scatters off a target $N(P)$ which is usually a nucleon or a nucleus at rest and with four momentum $P=P^{\mu}=(M, \overrightarrow{0})$, can simply be represented by

$$
l(p)+N(P) \rightarrow l\left(p^{\prime}\right)+X\left(P^{\prime}\right)
$$

where $l\left(p^{\prime}\right)$ and $X\left(P^{\prime}\right)$ represent the scattered lepton and the rest of the final state (which can have any number of particles) with four momenta $p^{\prime \mu}=\left(E^{\prime}, \vec{k}^{\prime}\right)$ and $P^{\prime \mu}=\left(E_{X}, \overrightarrow{k_{X}}\right)$ respectively. The scattering angle which is the angle between the incident and outgoing path/direction of the electron is denoted by $\theta$. The final (hadronic) state denoted by $x$ is not measured, with only the scattered electron detected and measured by the detector(s). In the first order (Born) approximation of the process, a virtual photon is exchanged (as depicted in Fig (1)) whose four momentum is equal to the difference between that of the incident and the scattered electron and is given by $\left(p-p^{\prime}\right)^{\mu}=(v, \vec{q})$, where $v=(P . q) / M$ and $\vec{q}$ represent the energy and 3 -momentum transferred by the incident electron to the target $N(P)$.

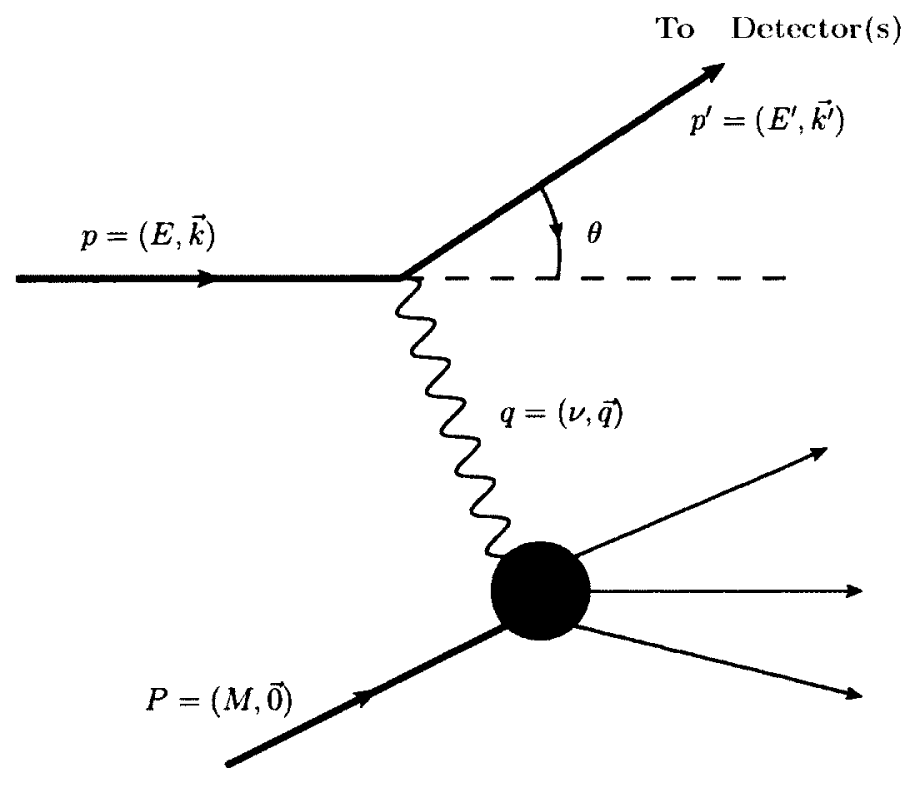

FIG. 1. Lowest order (Born approximation) Feynmann diagram representing the process of inclusive lepton scattering

The kinematics of the scattering process can be completely described in terms of two 
of the following Lorentz invariant variables.

$$
\begin{aligned}
v & =E-E^{\prime} \\
Q^{2} & =-q^{2} \simeq 4 E E^{\prime} \sin ^{2} \frac{\theta}{2} \\
W & =\sqrt{(P+q)^{2}}=\sqrt{M^{2}+2 M v-Q^{2}} \\
x & =\frac{Q^{2}}{2 P \cdot q}=\frac{Q^{2}}{2 M v} \\
y & =\frac{q \cdot P}{p \cdot P}=\frac{v}{E}
\end{aligned}
$$

where $Q^{2}=-q^{2}$ is the negative of the squared four-momentum transferred (with electron mass neglected in the expression for $Q^{2}$ ), which defines the resolution of the electron probe; $W$ is the invariant mass of the unmeasured final state $(x) ; x$ is known as the Bjorken scaling variable, which is also interpreted as the momentum fraction carried by the struck quark (parton) in the infinite momentum frame; $M$ is the nucleon mass $\approx 0.939 \mathrm{GeV}$, and lastly, $y$ is the fraction of the energy that is lost by the lepton during the process.

\subsubsection{DIFFERENTIAL CROSS SECTION AND STRUCTURE FUNCTIONS}

The differential cross section for the process of inclusive (polarized) electron scattering on (polarized) targets can be expressed, in the Born approximation, in terms of the product of leptonic tensor $L_{\mu v}$ and the hadronic tensor $W^{\mu \nu}$ as follows :

$$
\frac{d^{2} \sigma}{d \Omega d E^{\prime}}=\frac{\alpha^{2}}{Q^{4}} \frac{E^{\prime}}{E} L_{\mu v} W^{\mu v}
$$

where $\alpha=\frac{e^{2}}{4 \pi} \simeq 1 / 137$ is the electromagnetic fine structure constant.

The lepton tensor, which is calculable from QED, is given by:

$$
\begin{aligned}
L_{\mu v} & =\sum_{s^{\prime}} \bar{u}_{s}(p) \gamma_{\mu} u_{s^{\prime}}\left(p^{\prime}\right) \bar{u}_{s^{\prime}}\left(p^{\prime}\right) \gamma_{v} u_{s}(p) \\
& +2\left[p_{\mu} p_{v}^{\prime}+p_{\mu}^{\prime} p_{v}-g_{\mu v} p \cdot p^{\prime}+i \varepsilon_{\mu v \alpha \beta} s_{\alpha} q_{\beta}\right]
\end{aligned}
$$

where $u(p)$ are the Dirac spinors, $s_{\mu}=\bar{u} \gamma_{\mu} \gamma_{5} u$ is the lepton spin vector, $\varepsilon_{0123}=+1$ is the Levi-Civita tensor (using the special convention of [14]) and the sum is over all the unobserved final lepton spin states. This tensor can be can be considered as having two parts - symmetric (consisting of the first three terms) and antisymmetric (the last term) under the interchange of the indices $\mu, v$. The antisymmetric part vanishes if one uses an unpolarized lepton beam due to the averaging over the spins [15]. 
On the other hand, the hadron tensor characterizing the hadronic target is not calculable yet, due to the difficulties ${ }^{1}$ of fully solving the established theory (called QCD) for strong interacting objects. In this case, one needs to consider all the possible transitions that can happen from the target ground state $|N(P)\rangle$ to any of its excited states $\left|X\left(P^{\prime}\right)\right\rangle$. Using the completeness of the excited states, the hadronic tensor is given by:

$$
W_{\mu v}=\frac{1}{4 \pi M} \int d^{4} \zeta e^{i q \cdot \zeta}\left\langle N(s, P)\left|J_{\mu}(\zeta) J_{v}(0)\right| N(s, P)\right\rangle
$$

where $s$ denotes the target spin, $J_{\mu}(\zeta)$ is the electromagnetic current operator with $\zeta$ being the spatial four vector.

As with the lepton tensor, the hadronic tensor can also be further split into a symmetric and an anti-symmetric parts $W_{\mu \nu}=W_{\mu \nu}^{S}+W_{\mu \nu}^{A}$, with the two parts given by the following most general forms (as obtained from Lorentz and gauge invariance and parity conservation of the electromagnetic interaction):

$$
\begin{aligned}
W_{\mu \nu}^{S} & =W_{1}\left(v, Q^{2}\right)\left(\frac{q_{\mu} q_{v}}{q^{2}}-g_{\mu v}\right) \\
& +\frac{W_{2}\left(v, Q^{2}\right)}{M^{2}}\left(P_{\mu}-\frac{P \cdot q}{q^{2}} q_{\mu}\right)\left(P_{v}-\frac{P \cdot q}{q^{2}} q_{v}\right)
\end{aligned}
$$

and

$$
W_{\mu \nu}^{A}=i \varepsilon_{\mu v \alpha \beta} q^{\alpha}\left[G_{1}\left(v, Q^{2}\right) S^{\beta}+\frac{G_{2}\left(v, Q^{2}\right)}{M^{2}}\left(S^{\beta} P \cdot q-P^{\beta} S \cdot q\right)\right]
$$

where $S^{\mu}=\bar{u}(P) \gamma^{\mu} \gamma_{5} u(P) / 2 M$ is the spin vector for the hadron. This effectively parameterizes the the internal hadronic structure information into four response functions - two spin independent $\left(W_{1,2}\right)$ and two spin dependent $\left(G_{1,2}\right)$ functions, which are usually replaced by the following dimensionless structure functions:

$$
\begin{aligned}
& F_{1}\left(x, Q^{2}\right)=M W_{1}\left(v, Q^{2}\right) \\
& F_{2}\left(x, Q^{2}\right)=v W_{2}\left(v, Q^{2}\right) \\
& g_{1}\left(x, Q^{2}\right)=M v G_{1}\left(v, Q^{2}\right) \\
& g_{2}\left(x, Q^{2}\right)=v^{2} G_{2}\left(v, Q^{2}\right)
\end{aligned}
$$

\footnotetext{
${ }^{1}$ Due to the running of the coupling constant (a consequence of the unique QCD property known as the asymptotic freedom), the coupling between partonic constituents of the hadrons become very large, not allowing the perturbative method (the only "exact" method available so far) of solving QCD in the hadronic energy scale [12].
} 
The structure functions can be measured experimentally by using different combinations of beam and target polarizations. For example, one can extract the first two from the unpolarized scattering experiments because the total spin averaged differential cross section in the lab frame is related to the these unpolarized structure functions as follows:

$$
\frac{d^{2} \sigma}{d \Omega d E^{\prime}}=\left(\frac{d \sigma}{d \Omega}\right)_{P o i n t}\left(\frac{2}{M} F_{1}\left(x, Q^{2}\right) \tan ^{2} \frac{\theta}{2}+\frac{1}{v} F_{2}\left(x, Q^{2}\right)\right)
$$

with the Point cross section (for the lepton scattering from a Dirac particle - a spin-1/2 point particle of charge $+e$ ) given by

$$
\left(\frac{d \sigma}{d \Omega}\right)_{\text {Point }}=\frac{\alpha^{2} \cos ^{2} \frac{\theta}{2}}{4 E^{2} \sin ^{4} \frac{\theta}{2}} \frac{E^{\prime}}{E}
$$

with $\frac{E^{\prime}}{E}$ being the recoil factor.

The polarized structure functions $g_{1}$ and $g_{2}$ can, in principle, be separated by using different target spin orientations with respect to the beam direction and measuring two independent observables - the polarized cross-section differences $\Delta \sigma_{\|}$and $\Delta \sigma_{\perp}$ as given by the following equations. In the first case, the target spin is aligned along the beam direction and the cross-section difference is measured between anti-parallel and parallel target and electron spins.

$$
\Delta \sigma_{\|}=\frac{4 \alpha^{2}}{M v Q^{2}} \frac{E^{\prime}}{E}\left[\left(E+E^{\prime} \cos \theta\right) g_{1}\left(x, Q^{2}\right)-2 M x g_{2}\left(x, Q^{2}\right)\right]
$$

where

$$
\Delta \sigma_{\|}=\frac{d^{2} \sigma^{\downarrow \Uparrow}}{d \Omega d E^{\prime}}-\frac{d^{2} \sigma^{\uparrow \Uparrow}}{d \Omega d E^{\prime}}
$$

In the second case, a transversely polarized target with respect to the beam polarization is used, and the corresponding cross section difference (under the reversal of the target or beam spin direction) is related with the two spin structure functions as follows:

$$
\Delta \sigma_{\perp}=\frac{4 \alpha^{2}}{M v Q^{2}} \frac{E^{2}}{E}\left[g_{1}\left(x, Q^{2}\right)+\frac{2 E}{v} g_{2}\left(x, Q^{2}\right)\right] \sin \theta
$$

where

$$
\Delta \sigma_{\perp}=\frac{d^{2} \sigma^{\downarrow \Rightarrow}}{d \Omega d E^{\prime}}-\frac{d^{2} \sigma^{\uparrow \Rightarrow}}{d \Omega d E^{\prime}}
$$

Figures 2 and 3 show some of the past measurements of $g_{1}$ for proton. 


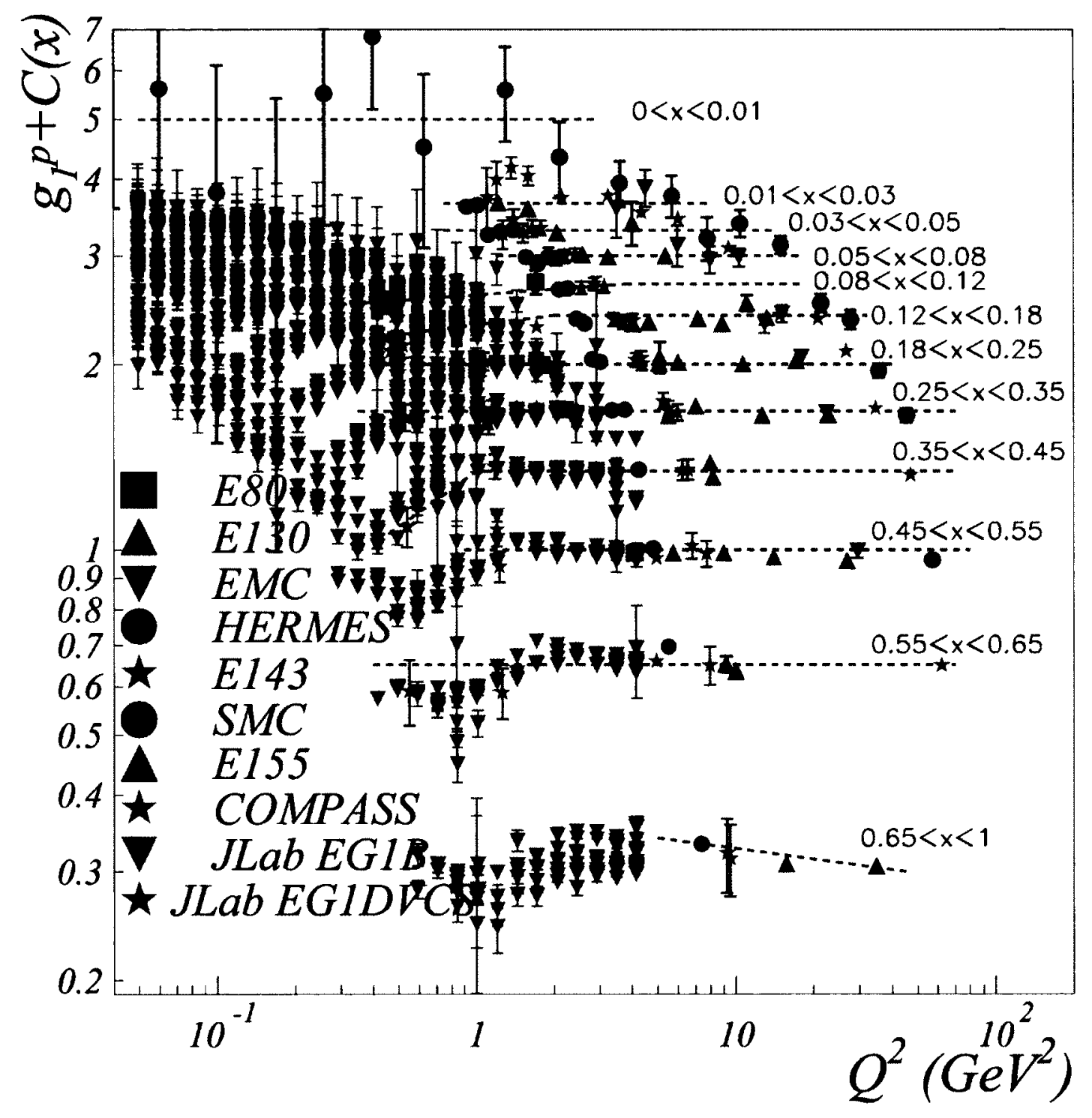

FIG. 2. $g_{1}^{p}$ data from various measurments (plot courtesy of A. Deur). 


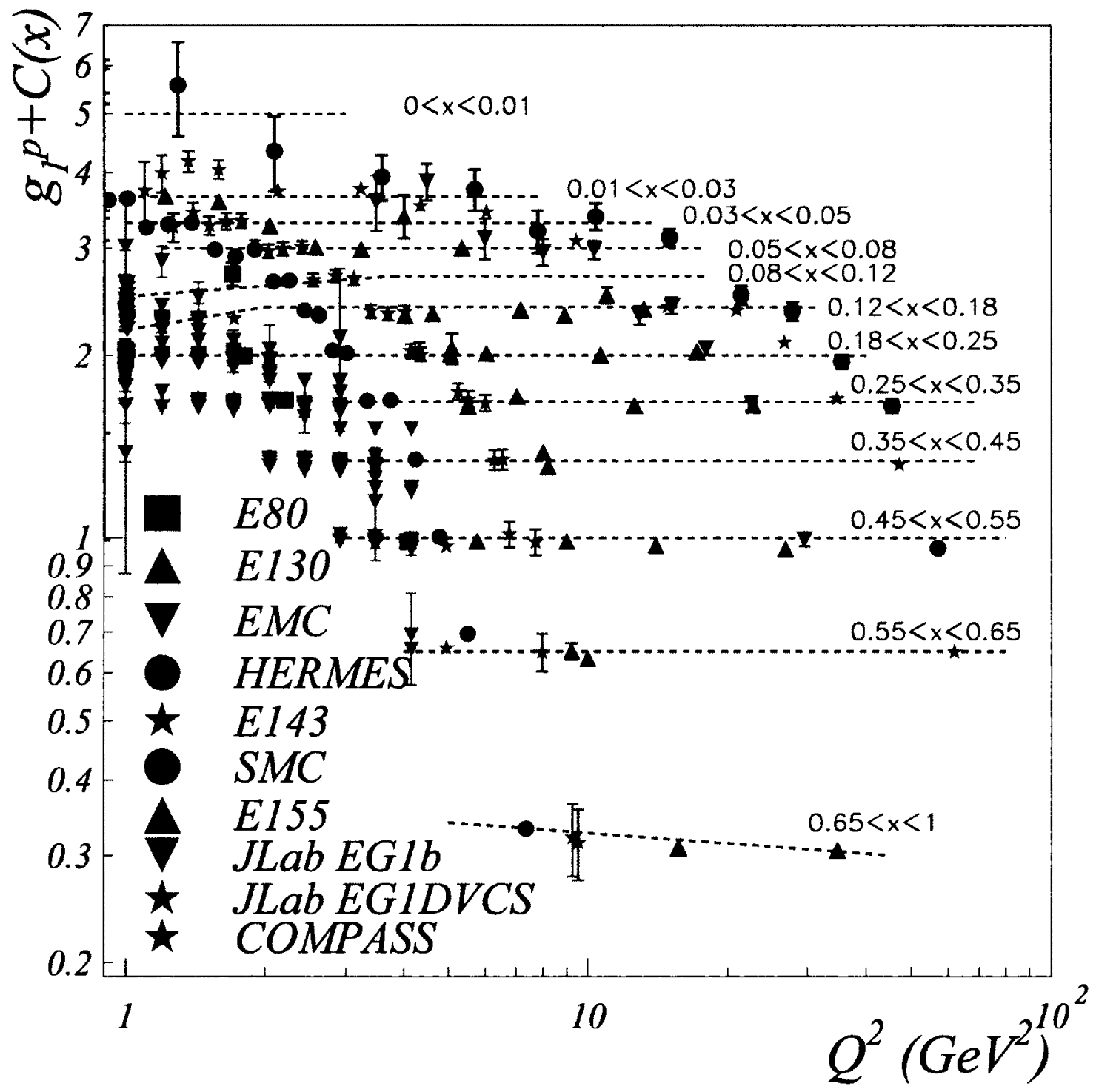

FIG. 3. $g_{1}^{p}$ data from various measurments (in the DIS region only) (plot courtesy of A. Deur). 


\subsubsection{VIRTUAL PHOTOABSORPTION CROSS SECTIONS}

We have seen in Sec. 2.1 that the lepton scattering can be viewed as the two step interaction process of the lepton with the target - first the emission of a virtual photon described by the Lepton tensor and then the absorption of the photon by the target as described by the hadronic tensor. The complete description is then obtained by the contraction of these tensors resulting in the inclusive differential cross-section, which can be expressed and described in terms of four structure functions. Equivalently, the process can be viewed as absorption of a virtual photon on the target and, therefore, the cross-section can also be expressed as virtual photo-absorption cross section in terms of four partial cross-sections $\sigma_{L}, \sigma_{T}, \sigma_{L T}$, and $\sigma_{T T}$ as follows:

$$
\frac{d^{2} \sigma}{d \Omega d E^{\prime}}=\Gamma\left[\sigma_{T}+\varepsilon \sigma_{L}-h P_{x} \sqrt{2 \varepsilon(1-\varepsilon)} \sigma_{L T}-h P_{z} \sqrt{1-\varepsilon^{2}} \sigma_{T T}\right]
$$

where $\mathrm{h}$ is the helicity of the polarized beam electron $(h= \pm 1$ for longitudinally polarized electrons) defined as

$$
\hat{h}=\frac{\vec{\sigma} \cdot \vec{p}}{|\vec{p}|}
$$

with $\vec{\sigma}$ and $\vec{p}$ being Pauli spin matrices and particle momentum respectively. Likewise, $P_{z}$ and $P_{x}$ are the target polarizations parallel and perpendicular to the virtual photon momentum $\vec{q} . \varepsilon$ is the longitudinal to transverse ratio of the exchanged virtual photon polarization

$$
\varepsilon=\left[1+2\left(1+\frac{v^{2}}{Q^{2}}\right) \tan ^{2} \frac{\theta}{2}\right]^{-1}
$$

and $\Gamma$ is the photon flux factor which is proportional to the photon flux $K$

$$
\Gamma=\frac{\alpha}{2 \pi^{2} Q^{2}} \frac{E^{\prime}}{E} \frac{K}{1-\varepsilon}
$$

Different conventions are used for virtual photon flux $K$. One given by Anselmino et al. [16] is:

$$
K_{A}=v
$$

In the Hand convention, the virtual photon spectrum is normalized using the equivalent photon energy [17]:

$$
K_{H}=\frac{W^{2}-M^{2}}{2 M}=v(1-x)
$$


Alternatively, Gilman's choice of the definition is [17]

$$
K_{G}=\left|\vec{q}_{l a b}\right|=\sqrt{v^{2}+Q^{2}}
$$

In the first convention, the flux is simply equal to the photon energy. In the Hand convention the flux is chosen to be equal to the equivalent photon energy in the center-of-mass frame and in Gilman's convention it is given by the photon momentum in the lab frame. In all cases, they become equal to $v$ at the photon point, and they also give numerically similar results in DIS but they are strongly convention dependent in the intermediate $Q^{2}$ region [15].

The partial cross-sections $\sigma_{L}, \sigma_{T}, \sigma_{L T}$, and $\sigma_{T T}$ are functions of $v$ and $Q^{2}$ among which the first two are cross sections for the absorption of longitudinally and transversely polarized photons respectively, while the latter two are the interference cross-sections which involve spin flips and can only be measured experimentally by double polarization methods. In the real photon limit $\left(Q^{2}=0\right), \sigma_{L}$ and $\sigma_{L T}$ vanish and the total photo-absorption cross-section becomes equal to $\sigma_{T}$ i.e. $\sigma(v)=\sigma_{T}(v)$.

The partial cross sections $\sigma_{T}$ and $\sigma_{T T}$ can, in turn, be expressed in terms of the helicity dependent photoabsorption cross sections $\sigma_{\frac{1}{2}}^{T}$ and $\sigma_{\frac{3}{2}}^{T}$ :

$$
2 \sigma_{T}=\sigma_{\frac{1}{2}}^{T}+\sigma_{\frac{3}{2}}^{T}, \quad 2 \sigma_{T T}=\sigma_{\frac{1}{2}}^{T}-\sigma_{\frac{3}{2}}^{T}, \quad \sigma_{L T}=\sigma_{T L}=\sigma_{\frac{1}{2}}^{L T}
$$

where the subscripts $1 / 2$ and $3 / 2$ indicate the total helicity projections of the photon and the target as illustrutated in the Fig. 4, whereas the superscript ' $T$ ' implies that the photons are transversely polarized (i.e., spin \pm 1 ).

As indicated at the beginning of this section, these photoabsorption cross sections are related to the four structure functions $\left(F_{1}, F_{2}, g_{1}\right.$ and $\left.g_{2}\right)$ of the target as follows:

$$
\begin{gathered}
\sigma_{T}=\frac{4 \pi^{2} \alpha}{M K} F_{1} \\
\sigma_{L}=\frac{4 \pi^{2} \alpha}{K}\left[\frac{F_{2}}{v}\left(1+\gamma^{2}\right)-\frac{F_{1}}{M}\right] \\
\sigma_{L T}=\frac{4 \pi^{2} \alpha}{M K} \gamma\left(g_{1}+g_{2}\right)
\end{gathered}
$$



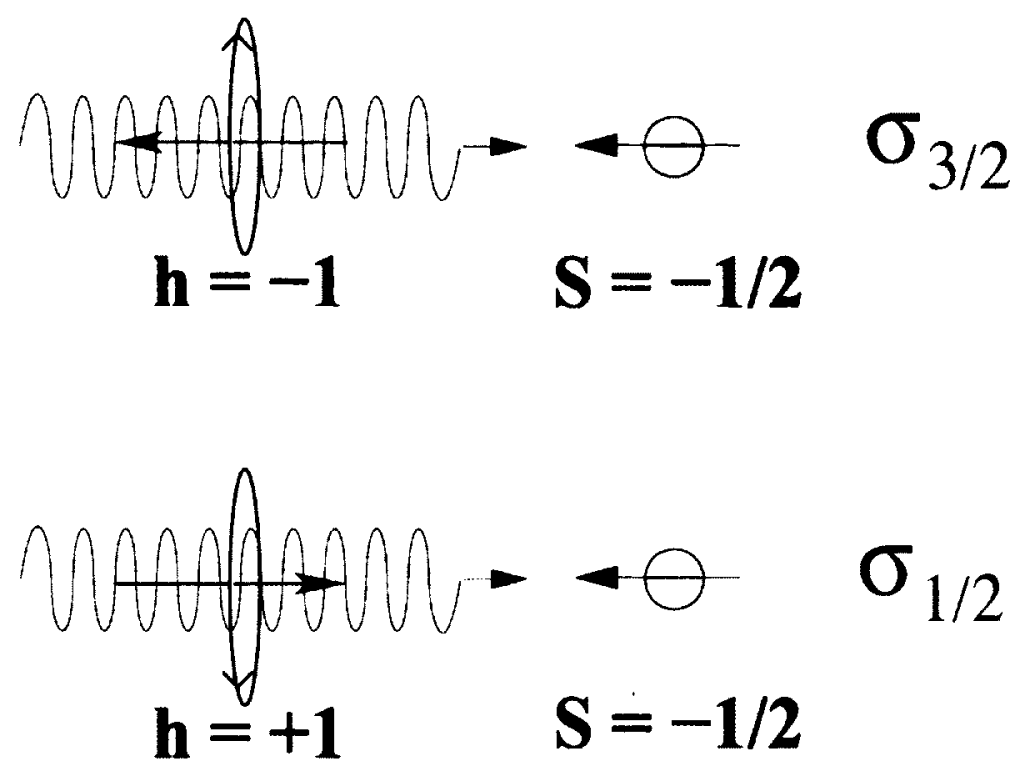

FIG. 4. Helicity of virtual photons (h) and target spin projections (S) corresponding to the helicity dependent photoabsorption cross sections $\sigma_{\frac{3}{2}}^{T}$ and $\sigma_{\frac{1}{2}}^{T}$ respectively

$$
\sigma_{T T}=\frac{4 \pi^{2} \alpha}{M K}\left(g_{1}-\gamma^{2} g_{2}\right)
$$

and, equivalently, the structure functions can be expressed in terms of the helicity amplitudes. For example, the relation for $g_{1}$ becomes as follows:

$$
g_{1}=\frac{M K}{8 \pi^{2} \alpha\left(1+\gamma^{2}\right)}\left(\sigma_{\frac{1}{2}}^{T}-\sigma_{\frac{3}{2}}^{T}+2 \gamma \sigma_{L T}\right)
$$

where $\gamma=Q / v$. Due to the earlier indicated convention dependent nature of the photon flux $K$, these relationships are also convention dependent and the interference terms can also be defined such that $\sigma_{L T(T T)}=-\sigma_{L T(T T)^{\prime}}^{\prime}$

\subsubsection{VIRTUAL PHOTON ASYMMETRIES}

Most of the past measurements of the spin structure functions come from measurements of asymmetries (defined below) rather than from direct measurements of cross sections because the asymmetries, being calculated from the ratios of measured counts, do not rely on the knowledge of detector acceptance, target thickness etc. The two experimental asymmetries measured in the electroproduction experiments are the "longitudinal" 
and "transverse" asymmetries defined as follows:

$$
\begin{aligned}
A_{\|}\left(x, Q^{2}\right) & =\frac{\Delta \sigma^{\uparrow \Uparrow}-\Delta \sigma^{\uparrow \Downarrow}}{\Delta \sigma^{\uparrow \Uparrow}+\Delta \sigma^{\uparrow \Downarrow}} \\
A_{\perp}\left(x, Q^{2}\right) & =\frac{\Delta \sigma^{\uparrow \Rightarrow}-\Delta \sigma^{\uparrow \Leftarrow}}{\Delta \sigma^{\uparrow \Rightarrow}+\Delta \sigma^{\uparrow \Leftarrow}}
\end{aligned}
$$

It is a common practice, for historical and practical reasons, to express these electroproduction asymmetries and structure functions in terms of the virtual photon asymmetries $A_{1}$ and $A_{2}$ given by:

$$
\begin{aligned}
& A_{1}\left(x, Q^{2}\right)=\frac{\sigma_{\frac{1}{2}}^{T}-\sigma_{\frac{3}{2}}^{T}}{\sigma_{\frac{1}{2}}^{T}+\sigma_{\frac{3}{2}}^{T}}=\frac{g_{1}\left(x, Q^{2}\right)-\gamma^{2} g_{2}\left(x, Q^{2}\right)}{F_{1}\left(x, Q^{2}\right)} \\
& A_{2}\left(x, Q^{2}\right)=\frac{2 \sigma_{\frac{1}{2}}^{T L}}{\sigma_{\frac{1}{2}}^{T}+\sigma_{\frac{3}{2}}^{T}}=\frac{\gamma\left[g_{1}\left(x, Q^{2}\right)+g_{2}\left(x, Q^{2}\right)\right]}{F_{1}\left(x, Q^{2}\right)}
\end{aligned}
$$

By using equations (30) through (34), we get the following expressions for the spin structure functions in terms of the two asymmetries and the unpolarized structure function $F_{1}$ :

$$
\begin{aligned}
& g_{1}\left(x, Q^{2}\right)=\frac{F_{1}\left(x, Q^{2}\right)}{1+\gamma^{2}}\left(A_{1}+\gamma A_{2}\right) \\
& g_{2}\left(x, Q^{2}\right)=\frac{F_{1}\left(x, Q^{2}\right)}{1+\gamma^{2}}\left(-A_{1}+\frac{A_{2}}{\gamma}\right)
\end{aligned}
$$

As their definitions indicate, the virtual photon asymmetries $A_{1}$ and $A_{2}$ have simple physical interpretations and $A_{1}$ can be directly measured, in principle, from real photon absorption measurements. But they are not directly accessible in the electroproduction data[5]. However, they can be extracted indirectly from the measured experimental asymmetries because the two types of asymmetries are related as follows:

$$
\begin{aligned}
& A_{\|}=D\left(A_{1}+\eta A_{2}\right) \\
& A_{\perp}=d\left(A_{2}-\xi A_{1}\right)
\end{aligned}
$$


where

$$
\begin{aligned}
D & =\frac{y\left[\left(1+\gamma^{2} y / 2\right)(2-y)-2 y^{2} m^{2} / Q^{2}\right]}{y^{2}\left(1-2 m^{2} / Q^{2}\right)\left(1+\gamma^{2}\right)+2(1+R)\left(1-y-\gamma^{2} y^{2} / 4\right)} \\
d & =\left[\frac{\left[1+\gamma^{2} y / 2\left(1+2 m^{2} y / Q^{2}\right)\right] \sqrt{1-y-\gamma^{2} y^{2} / 4}}{(1-y / 2)\left(1+\gamma^{2} y / 2\right)-y^{2} m^{2} / Q^{2}}\right] D \\
\eta & =\gamma \frac{\left[1-y-y^{2}\left(\gamma^{2} / 4+m^{2} / Q^{2}\right)\right]}{(1-y / 2)\left(1+\gamma^{2} y / 2\right)-y^{2} m^{2} / Q^{2}} \\
\xi & =\gamma \frac{1-y / 2-y^{2} m^{2} / Q^{2}}{1+\gamma^{2} y / 2\left(1+2 m^{2} y / Q^{2}\right)}
\end{aligned}
$$

Thus, the directly measurable quantities $A_{\|}$and $A_{\perp}$ are related to the spin structure functions through the virtual photon asymmetries, and hence provide a method to extract the spin structure functions. In practice, however, several of the past experiments have extracted $g_{1}$ by only measuring $A_{\|}$, with $g_{2}$ related part (which is small) either ignored or giving some parameterization input with an upper bound [18].

\subsubsection{TYPES OF INCLUSIVE SCATTERING}

While studying and discussing inclusive measurements, it is sometimes very useful to make distinctions between different kinematic regions defined in terms of the invariant mass (W) of the final state. Most often, three regions are recognized - elastic, quasi-elastic and inelastic. The inelastic region is further considered to have two kinematic regions - that of resonance production and the deep inelastic scattering (DIS) which is typically defined by $Q^{2}>1-2 \mathrm{GeV}^{2}$ and $W>2 \mathrm{GeV}$. These different regions are depicted in a typical cross section spectrum for inclusive scattering from a light nuclear target as shown in Fig 5. As one varies the transferred energy $v$ and momentum $Q^{2}$, different nucleon resonance peaks such as $\Delta, N_{1}^{*}$ and $N_{2}^{*}$ show up in the final state at specific values of invariant mass W. At low $Q^{2}$ values, a prominently tall but narrow peak shows up at $v=Q^{2} / 2 M_{T}$ (or equivalently at $W=W_{Q E}=\sqrt{M^{2}+Q^{2}\left(1-M / M_{T}\right)}$, where $M$ is the nucleon mass) due to the elastic scattering from the given target, and if it is a nuclear target, one more rather smeared out peak appears in between the elastic and resonance region due to the quasielastic scattering from the constituent nucleons of the target. In addition, excited nuclear states also show up in between the nuclear elastic and quasi-elastic peaks.

\section{Elastic Scattering}

Elastic scattering occurs when the target remains intact after the scattering, in other words it remains in the ground state and the transfered energy and momentum goes into supplying 


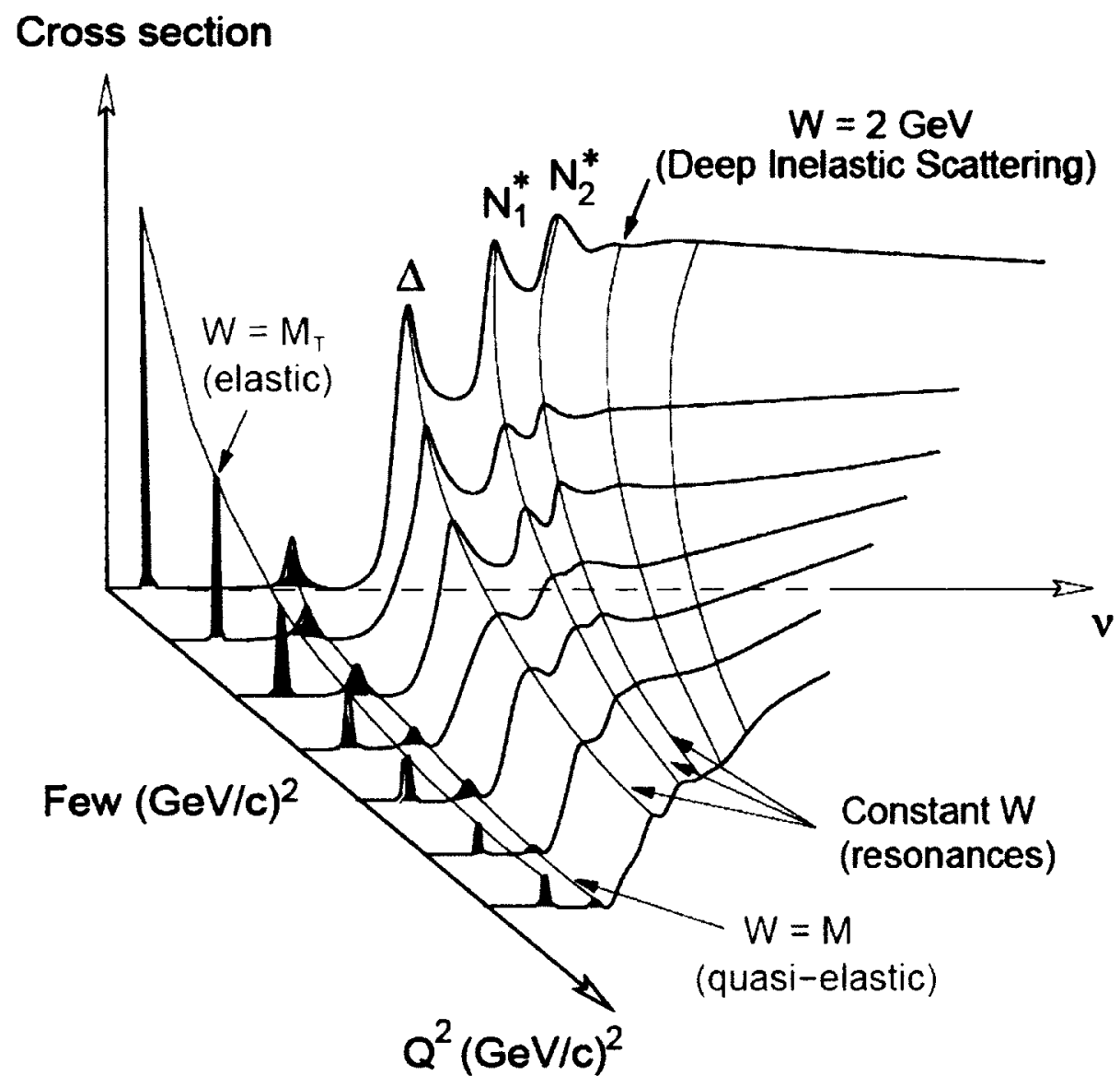

FIG. 5. Cross section (in arbitrary units) for the process of inclusive lepton scattering off a nuclear target (figure from [19]).

the kinetic energy of the target recoil. Because the final state entity represented by $X\left(P^{\prime}\right)$ above is simply the recoiling target, its invariant mass equals the target rest mass. This means, energy transfer $v=\frac{Q^{2}}{2 M_{T}}$, and the conservation of energy and momentum constrains the energy of the scattered electron $\left(E^{\prime}\right)$ to be directly correlated with the scattering angle $\theta$ :

$$
E^{\prime}=\frac{E}{1+\frac{2 E}{M_{T}} \sin ^{2} \frac{\theta}{2}}
$$

In other words, given the target mass and the beam energy, the kinematics of an elastic process can be completely described in terms of a single variable such as $\theta$ or $E^{\prime}$.

Because unpolarized elastic scattering is a special case of generic inclusive scattering, 
the differential cross section for the elastic process must also be a special case of the cross section (see Eq. (11)). Therefore, in the elastic limit, the response functions reduce to the combinations of the following Sachs form factors $\left(G_{E}\left(Q^{2}\right)\right.$ and $\left.G_{M}\left(Q^{2}\right)\right)$, also popularly known as the electric and magnetic form factors) as follows:

$$
\begin{aligned}
& W_{1} \Rightarrow \frac{Q^{2}}{4 M^{2}} G_{M}^{2}\left(Q^{2}\right) \\
& W_{2} \Rightarrow \frac{G_{E}^{2}\left(Q^{2}\right)+\frac{Q^{2}}{4 M^{2}} G_{M}^{2}\left(Q^{2}\right)}{1+\frac{Q^{2}}{4 M^{2}}}
\end{aligned}
$$

and the generic double differential cross section reduces to the following single differential cross section (because now, we have one independent variable) known as the Rosenbluth cross section:

$$
\frac{d \sigma}{d \Omega}=\left(\frac{d \sigma}{d \Omega}\right)_{\text {Point }}\left(\frac{G_{E}^{2}\left(Q^{2}\right)+\frac{Q^{2}}{4 M^{2}} G_{M}^{2}\left(Q^{2}\right)}{1+\frac{Q^{2}}{4 M^{2}}}+\frac{Q^{2}}{4 M^{2}} G_{M}^{2}\left(Q^{2}\right) \tan ^{2} \frac{\theta}{2}\right)
$$

The two Sachs form factors encode the information on the electric and magnetic charge (or equivalently electric current) distributions inside the target as seen through the scattering "probe" of resolution $Q^{2}$. These form factors for the nucleons must be normalized at $Q^{2}=0$ to their respective total charge and magnetic moments. Therefore, we get the following limiting values of the form factors in the units of the charge ' $e$ ' and the nuclear magneton $\mu_{N}=\frac{e \hbar}{2 M_{p}}$.

$$
\begin{array}{llll}
G_{E}^{p}(0)=e & \text { and } & G_{M}^{p}(0)=\mu_{p}=+2.793 \mu_{N} & \text { for proton } \\
G_{E}^{n}(0)=0 & \text { and } & G_{M}^{n}(0)=\mu_{n}=-1.913 \mu_{N} & \text { for neutron }
\end{array}
$$

It has been observed from the available measurements that magnetic form factors for both proton and neutron follow a dipole form over a significantly wide range of $Q^{2}$ (with deviations below $10 \%$ for $G_{M}^{p}$ in the $Q^{2} \leq 5 \mathrm{GeV}^{2}$ region) as given by

$$
\frac{G_{M}^{p}\left(Q^{2}\right)}{\mu_{p}}=\frac{G_{M}^{n}\left(Q^{2}\right)}{\mu_{n}}=G_{D}\left(Q^{2}\right)
$$

where $G_{D}$ is the dipole form as given by

$$
G_{D}\left(Q^{2}\right)=\left(\frac{\Lambda^{2}}{\Lambda^{2}+Q^{2}}\right)^{2}
$$

with $\Lambda=0.84 \mathrm{GeV}$. 
On the other hand, the proton electric form factor also follows the same form but only in the shorter $Q^{2} \leq 1 \mathrm{GeV}^{2}$ region, with significant deviation at higher $Q^{2}$. In other words, significant differences have been observed between the electric and magnetic form factors of the proton. The study of the $Q^{2}$ evolution of these form factors between the high and low $Q^{2}$ regions provides us information on the non-perturbative structure of the nucleon as well as some hints on the point in the energy scale from which the perturbative behavior begins to manifest.

Because the processes of polarized elastic and quasi-elastic scattering are well understood and their theoretical asymmetries are well determined, the polarized data collected for the processes can be used to reliably determine the luminosity times the product of beam and target polarizations $\left(P_{b} P_{t}\right)$.

\section{Quasi-elastic Scattering}

When the target is a nucleus with more than one nucleons, then there is some kinematic region where the electron penetrates the nucleus and scatters off one of the nucleons rather than off the whole nucleus. In such a process, the struck nucleon initially behaves as a nearly (quasi) free nucleon and gets knocked out of the nucleus after the interaction. In this case, the effective target mass as seen by the lepton becomes different from the overall target mass, and, because of the nuclear binding energy the effective nucleon (target) mass is also not exactly the same as the free nucleon mass either, thus changing the kinematics of the process to the effect of shifting the position of the quasi-elastic peak from the usual free nucleon elastic peak. In addition, the nucleons also have Fermi motion inside the nucleus, which has the effect of smearing out the energy and momentum distributions which is manifested in the broadening of the quasi-elastic peak.

For such processes, the Rosenbluth cross section is given by

$$
\frac{d^{2} \sigma}{d \Omega d E^{\prime}}=\left(\frac{d \sigma}{d \Omega}\right)_{\text {Mott }}\left\{\left(\frac{Q^{2}}{\vec{q}^{2}}\right)^{2} R_{L}+\left[\frac{1}{2}\left(\frac{Q^{2}}{\vec{q}^{2}}\right)^{2}+\tan ^{2} \frac{\theta}{2}\right] R_{T}\right\}
$$

where $R_{L}\left(v, Q^{2}\right)$ and $R_{T}\left(v, Q^{2}\right)$ are the response functions corresponding to the scattering/absorption of longitudinal and transverse virtual photon respectively.

\section{Resonances}

When the energy transfer in the scattering process increases beyond the point corresponding to the pion production threshold (i.e. when the combined invariant mass of the exchanged virtual photon and the target exceeds the value $W_{\pi}=M_{p}+m_{\pi} \approx 1.072 \mathrm{GeV}$ ), 
we leave the region of elastic or quasi-elastic scattering and enter the region of inelastic scattering. The region starts with a rich spectrum of nucleon excitations known as resonances. The existence of such excitation states provides further evidence that the nucleons are composite objects [20]. These resonances show up as different isolated or overlapping peaks in the measured scattering cross sections between the pion production threshold and the onset of deep inelastic scattering (about $2 \mathrm{GeV}$ in $W$ ), therefore this region is also sometimes known simply as the resonance region.

These resonances have been observed not only through the lens of lepton scattering but also through the absorption of photon and the scattering of hadron beams at different energies and so their properties have been studied using all of these types of experiments. It has now been well established that unlike the artificial width observed for the elastic peak as a result of the finite detector resolution and radiative effects, each of the resonances has a finite natural width (denoted by $\Gamma$ ) in its mass distribution (typically of over 100 $\mathrm{MeV}$ ), indicating (according to the uncertainty principle) that they are very short lived (the broader the widths, the shorter the lifetimes) [20]. As a result, these unstable particles quickly decay into other lighter particles (hadrons) such as pions and nucleons. Another consequence of this fact is that signals of some of the closely spaced resonances overlap, making it very difficult for them to be identified and investigated.

Right after the elastic or quasi-elastic peak in the $W$ spectrum of the cross sections, three prominent resonance related peaks are observed. The first peak corresponds to the $\Delta(1232)$ resonance with the number 1232 representing its rest mass (W) in units of $\mathrm{MeV}$.

Next comes the peak denoted by $N_{1}^{*}$, which consists of two closely spaced resonances $N^{*}(1520)$ and $N^{*}(1535)$. The third prominent peak denoted by $N_{2}^{*}$ is due to many resonances but at low $Q^{2}$, it is mainly due to $N^{*}(1680)$ which is the strongest in this kinematics. There also exists one resonance $N^{*}(1440)$ (also known as Roper resonance) between the $\Delta$ and the $N_{1}^{*}$ peaks.

In addition to these low lying resonances, several other higher resonances exist that can contribute to the cross sections measured but they cannot be isolated and measured using inclusive lepton scattering. These higher resonances have been observed and studied using different experimental and data analysis techniques, with varying levels of confidence in the experimental evidence for their existence. For example, [21] shows a complete list of the resonances observed or suggested so far, classified into two broad categories of $\mathrm{N}$-resonances and $\Delta$-resonances, where the main distinction is that the each of the $\mathrm{N}$ resonances has isospin $1 / 2$ (just like a nucleon), whereas the latter type of resonances all 
have isospins of $3 / 2$ (just as the prominent delta resonance).

By studying polarized scattering in the resonance region, one can learn about internal structure of nucleon resonances and their excitations. For example, the electromagnetic excitation of spin-3/2 resonances such as Delta occur mostly via M1 transitions and therefore the asymmetry $A_{1} \approx-0.5$, while the spin-1/2 resonances such as $\mathrm{S} 11$ have asymmetries

of $A_{1}=1$ because the spin-flip helicity amplitude $A_{\frac{3}{2}}^{T}$ cannot contribute. By studying the $Q^{2}$-dependence of the structure functions and the asymmetries in different parts of the resonance region, one can learn about the relative strengths of overlapping resonances, non-resonant background.

\section{Deep Inelastic Scattering (DIS)}

Looking at Fig (5), one can observe that as we go to higher energy transfer $v$ or the higher momentum transfer $Q^{2}$, the strengths of the resonances get weaker and after some point they get completely washed out. This "no-resonance" inelastic region, which is typically defined by $Q^{2}>1-2 \mathrm{GeV}^{2}$ and $W>2 \mathrm{GeV}$, is known as the deep inelastic scattering (DIS) region. In this case, the resolution of the virtual photon gets so sharp, it gets deep inside the nucleon and that it scatters off its constituents rather than from the whole target. In other words, the scattering cross section becomes an incoherent sum of the cross sections from different target constituents (partons). In this region, the cross sections and the structure functions depend only weakly on $Q^{2}[20]$ and depend mosly on the dimensionless variable $x=Q^{2} / 2 M v$. This behaviour of DIS is known as "scaling" phenomenon and the variable $x$ on which the DIS properties depend is known as the Bjorken scaling variable or simply as "Bjorken $x$ " (because the variable was introduced by James Bjorken in 1968). For example, Fig. (6) shows the $Q^{2}$-evolution of the $F_{2}$ structure function for the proton for different values of $\mathbf{x}$.

The scaling phenomenon for the structure function was previously predicted by Bjorken. The confirmation of the prediction by the DIS data from SLAC prompted Feynman to explain the behavior by proposing a partonic picture/model for the nucleons. In the model the nucleon (proton) is made up of point-like objects called partons (now identified as quarks and gluons). Because, in the DIS process, the lepton gets scattered off the point like partons rather than the finite sized target as a whole, the $Q^{2}$ dependence disappears because it is the finite size of the target which causes it to have have a form factor, thus introducing the $Q^{2}$-dependence in the measured cross sections (note the earlier discussed dipole form for the $Q^{2}$ dependence of the form factors). 


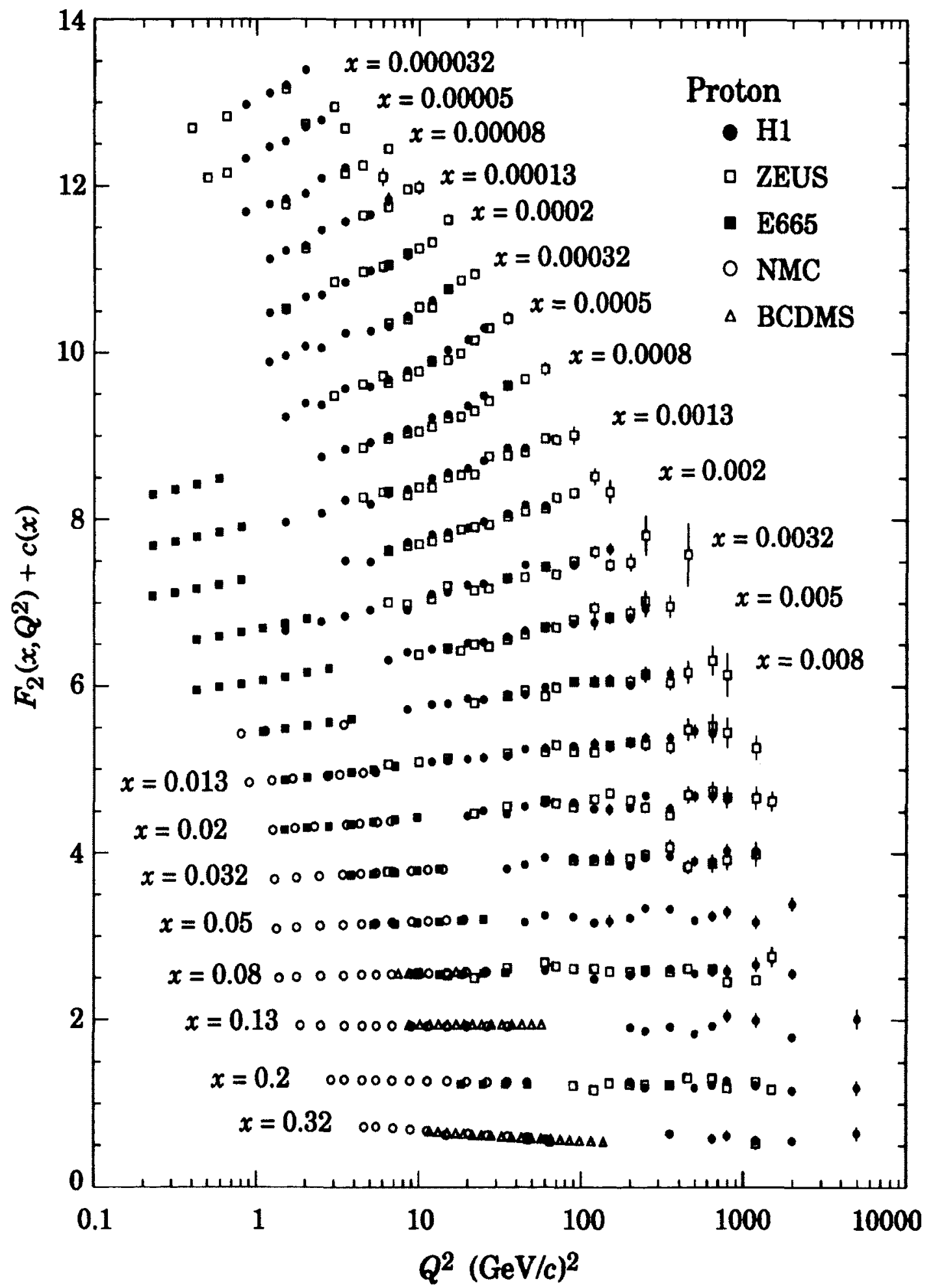

FIG. 6. The $F_{2}^{p}$ structure function showing the approximate scaling behaviour in DIS). 
Another important observation from the DIS results was that the ratio of the two unpolarized structure functions $F_{1}$ and $F_{2}$ satisfy the following Callan-Gross relation [20]:

$$
F_{2}(x)=2 x F_{1}(x)
$$

These two important observations led to the following profoundly important conclusions about the nucleon strucuture:

- The fact that the scaling behaviour is observed experimentally gives a strong evidence for the nucleon as being made up of point like charged particles (now identified with the quarks).

- Because, it is expected theoretically that the Callan-Gross relation holds true only for the scattering of spin-1/2 Dirac particles, the experimental observation of this behaviour confirms that the point-like constituents of the nucleon must be spin$1 / 2$ particles.

To simplify the interpretation of the DIS results, a carefully chosen frame of reference (dubbed the infinite momentum frame), in which $v$ and $Q^{2}$ go to infinity, is used to formulate the the parton model ${ }^{2}$. If the proton is observed from such a fast moving system, then one can ignore the transverse momenta and the rest masses of the constituents, allowing the target structure to be expressed, to a first approximation ${ }^{3}$, by the longitudinal momenta of its constituents. This gives a direct interpretation of the Bjorken scaling variable as the fraction of the proton's four-momentum which is carried by the struck parton. In other words, the virtual photon of four momentum $q=(v / c, \vec{q})$ (measured in lab frame) interacts with a parton of four momentum $x P$, where $P$ is the proton's overall four momentum. (One caveat about this is that, strictly speaking, this interpretation is valid only in the limit $Q^{2} \rightarrow \infty$ ) [20]. The nucleon cross section then becomes the simple incoherent sum of the individual parton cross sections with the latter weighted by their respective parton number densities as well as by the squares of their charges (because the process occurs through

\footnotetext{
${ }^{2}$ It should be remembered that the physics of any process doesn't change with the choice of the reference frame. Any frame can be chosen for the convenience of the description without affecting the Physics process

${ }^{3}$ This approximation is known as the impulse approximation (IA), because in the interaction time between the photon and the struck parton is so short that, in this fast moving frame the interaction between the partons themselves seem safely negligible, thus allowing the DIS process to be viewed as an incoherent sum of the elastic scatering from its non-interacting constituents. The validity of the impulse approximation in DIS is also helped by the fact that the parton-parton interaction at short distances is weak due to the property of the interaction known as the "asymptotic freedom".
} 
the electromagnetic interaction (the weak interaction ignored all along)). As a result, the structure functions take the following forms:

$$
\begin{aligned}
& F_{1}(x)=\frac{1}{2} \sum_{f} e_{f}^{2} q_{f}(x)=\frac{1}{2} \sum_{f} e_{f}^{2}\left[q_{f}^{\uparrow}(x)+q_{f}^{\downarrow}(x)\right] \\
& g_{1}(x)=\frac{1}{2} \sum_{f} e_{f}^{2} \Delta q_{f}(x)=\frac{1}{2} \sum_{f} e_{f}^{2}\left[q_{f}^{\uparrow}(x)-q_{f}^{\downarrow}(x)\right]
\end{aligned}
$$

where $q_{f}(x)$ and $\Delta q_{f}(x)$ are known as the unpolarized and polarized parton distribution functions for a parton of flavor $\mathrm{f}$, with the the function $q_{f}(x)$ representing the probability of finding a certain number $q_{f}(x)$ of partons of flavor ' $f$ ' at a given value of $x$ [5] (in other words, the integral of $q_{f}(x)$ and $x q_{f}(x)$ over the complete range of $x$ gives us the total number of quarks and the total momentum carried by the quarks of the particular flavor ' $f$ ' (i.e. up, down, strange etc)). Likewise, $\Delta q_{f}(x)$ being the difference between the distributions of $q_{f}^{\uparrow}(x)$ and $q_{f}^{\downarrow}(x)$, it gives the probability of finding the number of partons with net spin aligned along the nucleon helicities minus the antialigned. Since $F_{1}(x)$ and $F_{2}(x)$ are related via the Callan-Gross relation, $F_{2}(x)$ can similarly expressed and interpreted in terms of the parton distribution functions, but because of the lack of similar simple relation between $g_{1}(x)$ and $g_{2}(x)$, there is no simple intuitive interpretation of $g_{2}(x)$ in the quark-parton model. But from the study of operator product expansion (OPE) method (see the next chapter), it is revealed that in addition to a $g_{1}$ related part, the $g_{2}$ structure function also has so-called "higher-twist" part which carries information on quark-gluon interactions that occur inside the nuclon [18].

\section{$Q^{2}$ Dependence of Structure Functions}

Finally, it is worthwhile to note that the Bjorken scaling observed in the DIS data is only an approximation, and the scaling law is obeyed in the strict sense only in the asymptotically free kinematics of $Q^{2} \rightarrow \infty$. In the DIS region, the structure functions show a slow logarithmic $Q^{2}$ dependence, and the dependence gets stronger at lower $Q^{2}$. There are, in total, four sources for the scaling violation or the $Q^{2}$ dependence: 1) gluon radiation, 2) scale dependence of the parton distribution functions due to DGLAP evolution, 3) higher twist contributions, and 4) $Q^{2}$ dependence of the amplitudes for different resonant excitations. The first two sources are dominant in the DIS region and the other two are negligible, whereas the latter two become dominant when one moves away from the DIS region. 
(Anti)quarks can radiate gluons (similar to QED radiative effects) before and after the scattering (and these gluons, in turn, can tum into quark-antiquark or gluon-gluon pairs), thus resulting in a logarithmic $Q^{2}$ dependence of structure functions. In addition, the coupling constant $\left(\alpha_{s}\right)$, which is used as the expansion parameter to get the pQCD corrections, is also $Q^{2}$ dependent (also known as the "running" of $\alpha_{s}$ ). This $Q^{2}$ variation of the structure functions is referred to as QCD evolution, which is described by the DGLAP equations as developed by Dokshitzer, Gribov, Lipatov, Altarelli and Parisi $[22,23,24]$ in the form of the $Q^{2}$-evolution of the parton distribution functions. The significance of such evolution equations is that once the parton distributions are known at one scale or at point in kinematics, then these equations can be used to calculate the distributions at any other scale where the perturbative QCD is applicable.

In addition to the logarithmic scaling violations due to the two sources which are dominant in the DIS regime, corrections also arise due to multi-parton correlations in the nucleon which gives rise to terms that are proportional to different powers of $1 / Q^{2}$. These corrections are relatively small at large $Q^{2}$ but are expected to be large and non-negligible in the low $Q^{2}$ region. These contributions are represented by all the non-leading order terms in the power series expansion in terms of $1 / Q^{2}$ and are known as the higher twist corrections in the language of Operator Product Expansion (discussed later in Sec. 2.3.2). Finally, the resonance excitations themselves have different $Q^{2}$ dependent excitaion amplitudes due to the different kinematics dependent excitation mechanisms (electric, magnetic, Columb/scalar) and their contributions to the structure functions make the latter $Q^{2}$ dependent as well.

\subsection{MOMENTS OF $g_{1}$ AND SUM RULES}

Moments of structure functions are their integrals (over the complete $\mathrm{x}$ range) weighted by various powers of the variable $\mathrm{x}$. The $n^{t h}$ moment of $g_{1}$, for example, is given by

$$
\Gamma_{n}\left(Q^{2}\right)=\int_{0}^{1} g_{1}\left(x, Q^{2}\right) x^{(n-1)} d x
$$

The moments allow the studies of the ( $Q^{2}$ dependence of $)$ fundamental properties of nucleon structure. For example, the first moment of $x F_{1}$ of a nucleon gives the total momentum or mass fraction carried by quarks and the first moment of $g_{1}$ gives the fraction of the nucleon spin contributed by the quark helicities. These integrals get their particular significance from the fact that they can be predicted from rigorous theoretical methods, 
such as in the sum rules derived from general assumptions or from the method of Operator Product Expansion, lattice QCD calculations and $\chi$ PT calculations ${ }^{4}$ (see Sec. 2.3). Their importance can be highlighted from the fact that it was the experimental tests of the sum rules involving the first moments of nucleon that led to the discovery of the original "spin crisis" and provided a significant test of QCD in the spin sector [18].

In this section, three integrals are considered which have been calculated from the EG4 data on deuteron - the first moment of $g_{1}\left(\Gamma_{1}\right)$, the generalized GDH integral $\left(\bar{I}_{T T}\right)$, and the generalized forward spin polarizability $\left(\gamma_{0}\right)$.

\subsubsection{FIRST MOMENT $\Gamma_{1}$ OF $g_{1}$}

The first moment of $g_{1}$ is the integral of $g_{1}$ over the complete range of the Bjorken scaling variable $\mathrm{x}$.

$$
\Gamma_{1}\left(Q^{2}\right)=\int_{0}^{1} g_{1}\left(x, Q^{2}\right) d x
$$

This moment gives, in the quark-parton model, the fraction of the nucleon spin contributed by the quark helicities and enters directly into two historically important sum rules - Ellis-Jaffe sum rule and Bjorken sum rule. Measurements of the moment on the proton by the European Muon Collaboration (EMC) in 1988 showed that the Ellis-Jaffe sum rule is violated, which meant that the long held belief that all the proton spin is carried by quarks is not true, thus, sparking the well known "spin crisis". On the other hand, measurements from SLAC, CERN, Fermilab, DESY, and more recently, from JLab, have confirmed the Bjorken sum rule (which relates the difference of the first moments of the proton and the neutron to the fundamental axial coupling constant $\left(g_{A}\right)$ of neutron beta decay) at the level of $10 \%$ accuracy, thus helping establish the QCD as the correct theory of the strong interactions. The moment also enters into the virtual photon extension of another famous sum rule - the GDH sum rule (see below).

In addition, the moment is studied on its own right because it provides a powerful tool to test the validity of various theories and models in which it is calculable. In the past, it has been measured on proton, deuteron and neutron $\left({ }^{3} \mathrm{He}\right)$ at SLAC, CERN and DESY in the DIS region in order to understand the quark spin contribution as well as to test the validity of the Bjorken sum rule and hence QCD as a result [18]. Recently, it has also been

\footnotetext{
${ }^{4}$ In contrast, the same is not true about the structure functions because presently their complete description based on QCD first principles has not been possible yet (especially in the low to intermediate momentum transfer regions due to the strong coupling property of QCD).
} 
measured at JLab from DIS down to a fairly low $Q^{2}$ region. In the intermediate and low momentum transfers, some phenomenological model predictions are available, whereas in the very low $Q^{2}$ region, many chiral perturbation theory $(\chi \mathrm{PT})$ calculations are available. Fig. 7 shows some of these calculations along with the past measurements from SLAC and from the EGlb experiment at JLab.

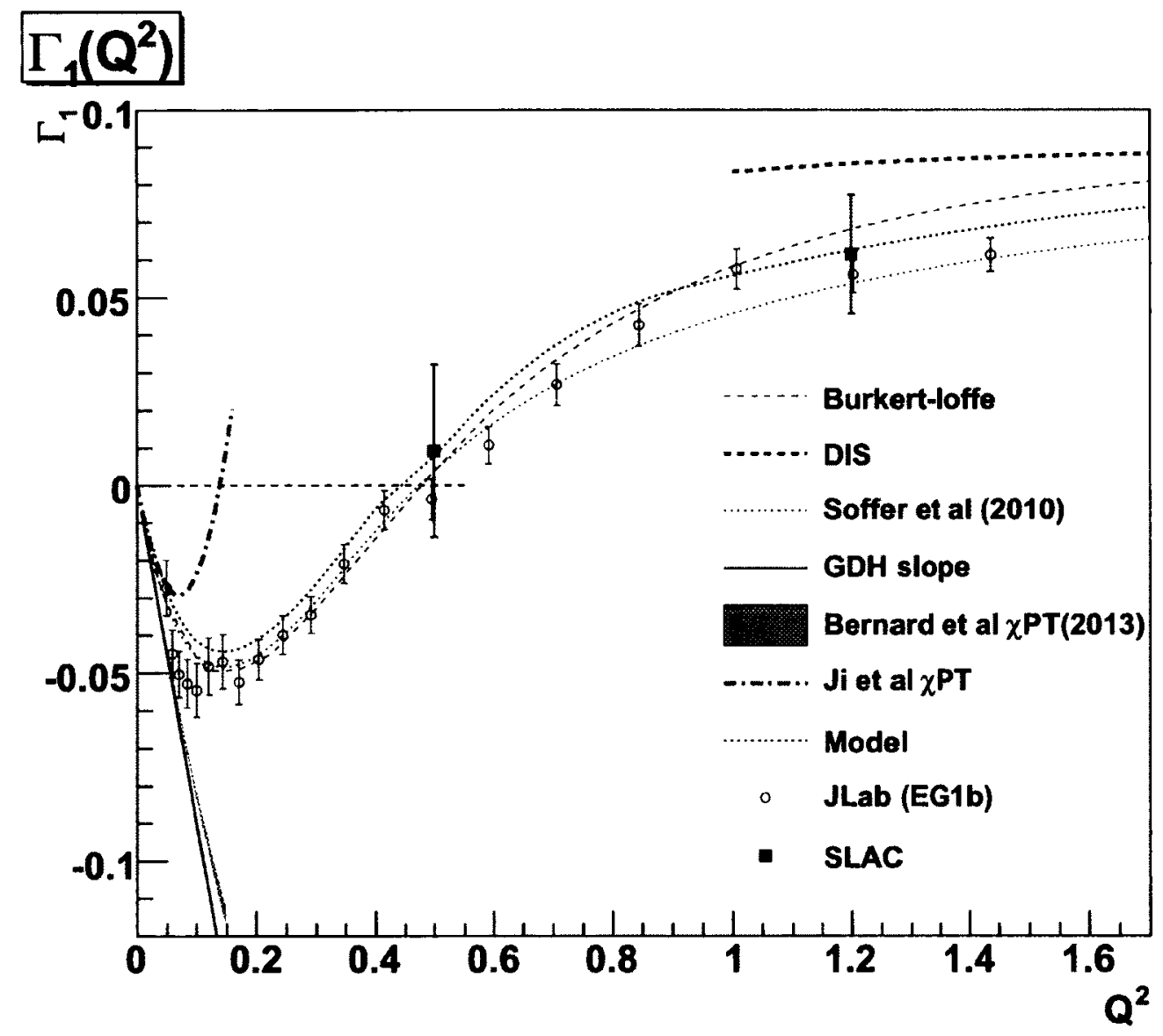

FIG. 7. Some theoretical predictions for $\Gamma_{1}^{d}$ and some data from past measurements. The theories and models which make these predictions are described in Sec. 2.3. 


\subsubsection{GENERALIZED GDH INTEGRAL}

\section{GDH Sum Rule}

The Gerasimov-Drell-Hearn (GDH) sum rule $[25,26]$ relates the energy weighted sum of a particle's photo-absorption cross sections to its anomalous magnetic moment $\kappa$. For a target of arbitrary spin S, the sum rule is:

$$
\int_{v_{t h}}^{\infty} \frac{\sigma_{P}(v)-\sigma_{A}(v)}{v}=-4 \pi^{2} \alpha S\left(\frac{\kappa}{M}\right)^{2}
$$

where $\sigma_{P}$ and $\sigma_{A}$ are the photoabsorption cross sections with photon helicity parallel and anti-parallel to the target spin respectively. $M$ and $k$ represent the target mass and anomalous magnetic moment respectively and $\mathrm{S}$ represents the target spin. The integration extends from the onset $v_{t h}$ of the inelastic region ${ }^{5}$ through the entire kinematic range and is weighted by the inverse of the photon energy $v$.

The sum rule was derived (see App. A) in the late 1960s based on some very general assumptions as follows:

1. Lorentz and gauge invariance in the form of the low energy theorem of Low, Goldman and Goldberger

\section{Unitarity in the form of the optical theorem}

3. Causality in the form of an unsubtracted dispersion relation for forward Compton scattering.

The sum rule for the proton has been measured (at various places such as Mainz, Bonn, BNL and others) and verified to within $10 \%[27,28,29,30]$, whereas there is little data on neutron and other targets.

Implications of the sum rule The sum rule relates the static property $\kappa$ of a particle's ground state with the sum of the dynamic properties of all the excited states. One deeper significance of this sum rule is that if a particle has a non-zero anomalous magnetic moment, then it must have some internal structure, and, therefore, a finite size, in order to have the excited states (a point-like particle cannot have excited states). Because of the

\footnotetext{
${ }^{5}$ The pion production threshold given by $v_{t h}=m_{\pi}\left(1+m_{\pi} / 2 M\right) \approx 150 \mathrm{MeV}$ marks the onset of the inelastic region for the nucleons, but for nuclei, the summation starts from the first nuclear excitation level
} 
same reason, the discovery of nucleon anomalous magnetic moments provided one of the first strong indications that the nucleons had some intrinsic internal structure.

In addition to the benefit of that implication, the sum rule and its extension to $Q^{2}>0$ provides an important testing ground for various theoretical predictions based on QCD and its effective theories/models.

\section{Generalization of the GDH Sum (Rule)}

In order to investigate the "spin crisis" of the 1980's, Anselmino et al. [31] proposed that the real photon $\left(Q^{2}=0\right) \mathrm{GDH}$ integral could be extended to electroproduction cross sections (finite $Q^{2}$ ) and that the experimental determination of the extended integral would shed light on the transition from the perturbative to non-perturbative QCD. The idea was to use the virtual photoabsorption cross sections in place of the real photoabsorption cross sections and proceed in exactly the same way as when deriving the real photon GDH sum rule. This extension depends somewhat on the choice of the virtual photon flux (see Sec. 2.1.3), and on how the spin structure function $g_{2}$ is considered [32]. In one extension the virtual photon flux given by $K=v$ (see Eq. 27) is chosen and the real photoabsorption cross section difference in Eq. 62 are replaced by the corresponding virtual photoabsorption cross section difference $2 \sigma_{T T}$ as given by Eq. 30. With the use of Eq. 34, and some algebraic manipulation, we get the following extended GDH integral (considering only the inelastic contribution starting from the pion production threshold) [18]

$$
\bar{I}_{T T}=\frac{2 M^{2}}{Q^{2}} \int_{0}^{x_{0}\left(Q^{2}\right)} d x\left[g_{1}\left(x, Q^{2}\right)-\frac{4 M^{2} x^{2}}{Q^{2}} g_{2}\left(x, Q^{2}\right)\right]
$$

where $x_{0}\left(Q^{2}\right)=Q^{2} /\left(Q^{2}+m_{\pi}\left(2 M+m_{\pi}\right)\right)$ is the pion production threshold that defines the onset of the inelastic region.

Using Eq. 38, the integral can also be expressed in terms of the first moment of the product $A_{1} F 1$ as follows:

$$
\bar{I}_{T T}=\frac{2 M^{2}}{Q^{2}} \int_{0}^{x_{0}\left(Q^{2}\right)} d x A_{1}\left(x, Q^{2}\right) F_{1}\left(x, Q^{2}\right)
$$

Fig. 8 shows a $\chi$ PT prediction along with the integral calculated from the model used in the EG4 data analysis covered by this thesis (see below). As is evident from the figure, the limiting value of the integral as $Q^{2}$ goes to zero is $\bar{I}_{T T}(0)=-1.5897$ 


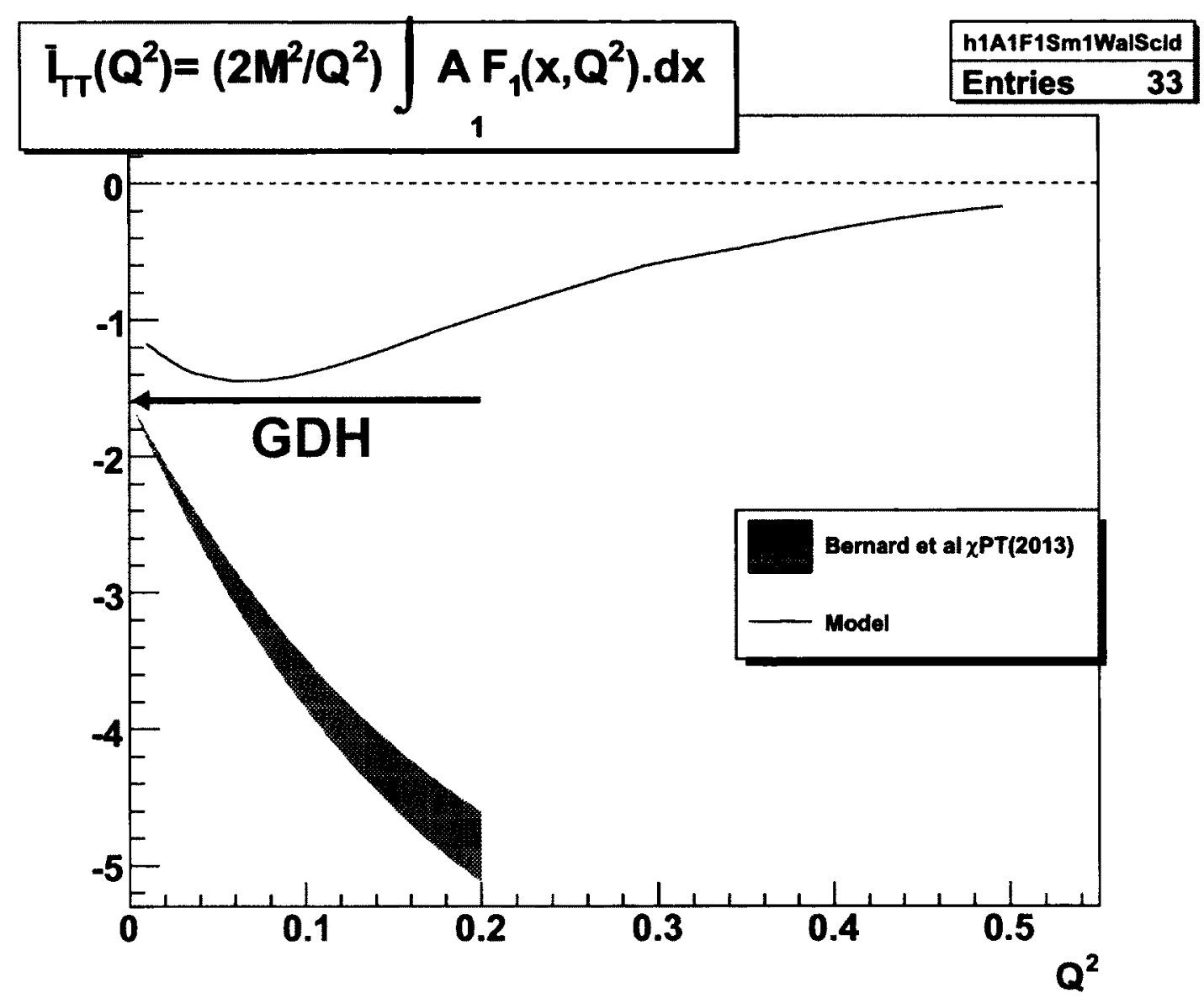

FIG. 8. A $\chi$ PT theoretical predictions for $\bar{I}_{T T}^{d}$ along with the integral calculated from the model used in the simulation for the data analysis.

\subsubsection{GENERALIZED FORWARD SPIN POLARIZABILITY $\gamma_{0}$}

Polarizabilities are fundamental observables (quantities) that characterize the structure of composite objects such as nucleons or deuteron. They reflect the response to external perturbations such as external electromagnetic fields. Like the GDH sum, they are also integrals over the excitation spectrum of the target and their derivations rely on the same basic assumptions. At the real photon point, for example, the electric and magnetic polarizabilities $\alpha$ and $\beta$ represent the target's response to external electric and magnetic fields respectively. The generalized polarizabilities represent the extensions of these quantities to the case of virtual photon Compton scattering. Because the integrals defining the polarizabilities involve weighting by some powers of $1 / v$ or $x$, they converge faster than the 
first moments and thus are more easily determined from low energy measurements. In other words, they have reduced dependence on the extrapolations to the unmeasured regions at large $v$, and higher sensitivity to the low energy behavior of the cross sections (particularly the threshold behavior), thus providing better testing grounds for theoretical predictions such as from $\chi \mathrm{PT}$ and phenomenological models [32].

The GDH sum rule comes from (see App. A) the first term of the low energy expansion of the forward Compton amplitude [33]. Likewise, we get another sum rule from the second, i.e., the next-to-leading term (which is in the third power of $v$ ). The second coefficient of the expansion is known as the forward spin polarizability $\gamma_{0}$ and by comparing the coefficients of the $v^{2}$ terms on both sides (coming from the dispersion relations on the left side and from the low energy expansion on the right side) gives us the following expression for the polarizability [34]:

$$
\gamma_{0}=-\frac{1}{4 \pi^{2}} \int_{t h r}^{\infty} \frac{\sigma_{\frac{1}{2}}-\sigma_{\frac{3}{2}}}{v^{3}} d v
$$

Now, by considering the case of forward scattering of a virtual photon and using the same general approach as for getting the generalized GDH sum rule, the $\mathscr{O}\left(v^{3}\right)$ (NLO) term in the low energy expansion of VVCS (doubly virtual Compton scattering) amplitude $g_{T T}\left(x, Q^{2}\right)$ gives the following generalization of the forward spin polarizability [17] [18]:

$$
\begin{aligned}
\gamma_{0}\left(Q^{2}\right) \equiv \gamma_{T T}\left(Q^{2}\right) & =\frac{16 \alpha M^{2}}{Q^{6}} \int_{0}^{x_{0}}\left[g_{1}\left(x, Q^{2}\right)-\frac{4 M^{2} x^{2}}{Q^{2}} g_{2}\left(x, Q^{2}\right)\right] x^{2} d x \\
& =\frac{16 \alpha M^{2}}{Q^{6}} \int_{0}^{x_{0}} A_{1}\left(x, Q^{2}\right) F_{1}\left(x, Q^{2}\right) x^{2} d x
\end{aligned}
$$

where $\alpha=\frac{e^{2}}{4 \pi}$ is the fine structure constant. At large $Q^{2}$, the $g_{2}$ dependent term in the integrand becomes negligible and $\gamma_{0}$ reduces to the third moment of $g_{1}[17]$.

In exactly the same manner, from the $\mathscr{O}\left(v^{2}\right)$ term of the low energy expansion of the VVCS amplitude $g_{L T}\left(x, Q^{2}\right)$ one gets another polarizability - the generalized longitudinaltransverse polarizability as follows:

$$
\delta_{0}\left(Q^{2}\right) \equiv \delta_{L T}\left(Q^{2}\right)=\frac{16 \alpha M^{2}}{Q^{6}} \int_{0}^{x_{0}}\left[g_{1}\left(x, Q^{2}\right)+g_{2}\left(x, Q^{2}\right)\right] x^{2} d x
$$

But, this latter polarizability is not considered in this thesis.

Because the generalized polarizabilities can be expressed with the moments of the structure functions, it is possible to measure them using measurements of the structure functions. As stated earlier, because of the weighting by some powers of $v$ or $x$, these 
integrals converges more rapidly in energy than the GDH integral and therefore can more easily be determined by low beam energy measurements. These integrals are valuable because they shed light on the long distance (soft), non-perturbative aspects of the target structure. The integrals are possible to be calculated using effective or approximate theories such as $\chi \mathrm{PT}$ and lattice methods. Thus the measurements of these quantities provide benchmark tests of such theories.

The first measurement of this quantity for a proton target at the real photon point was done by the GDH experiment at Mainz [34]. Recently the JLab EGlb experiment has provided some finite $Q^{2}$ results for both deuteron (see Fig. 9) as well as nucleon targets [35]. Some $\chi$ PT calculations [36] [37] as well as phenomenological predictions [38] are also available and have been used to compare with the available measurments.

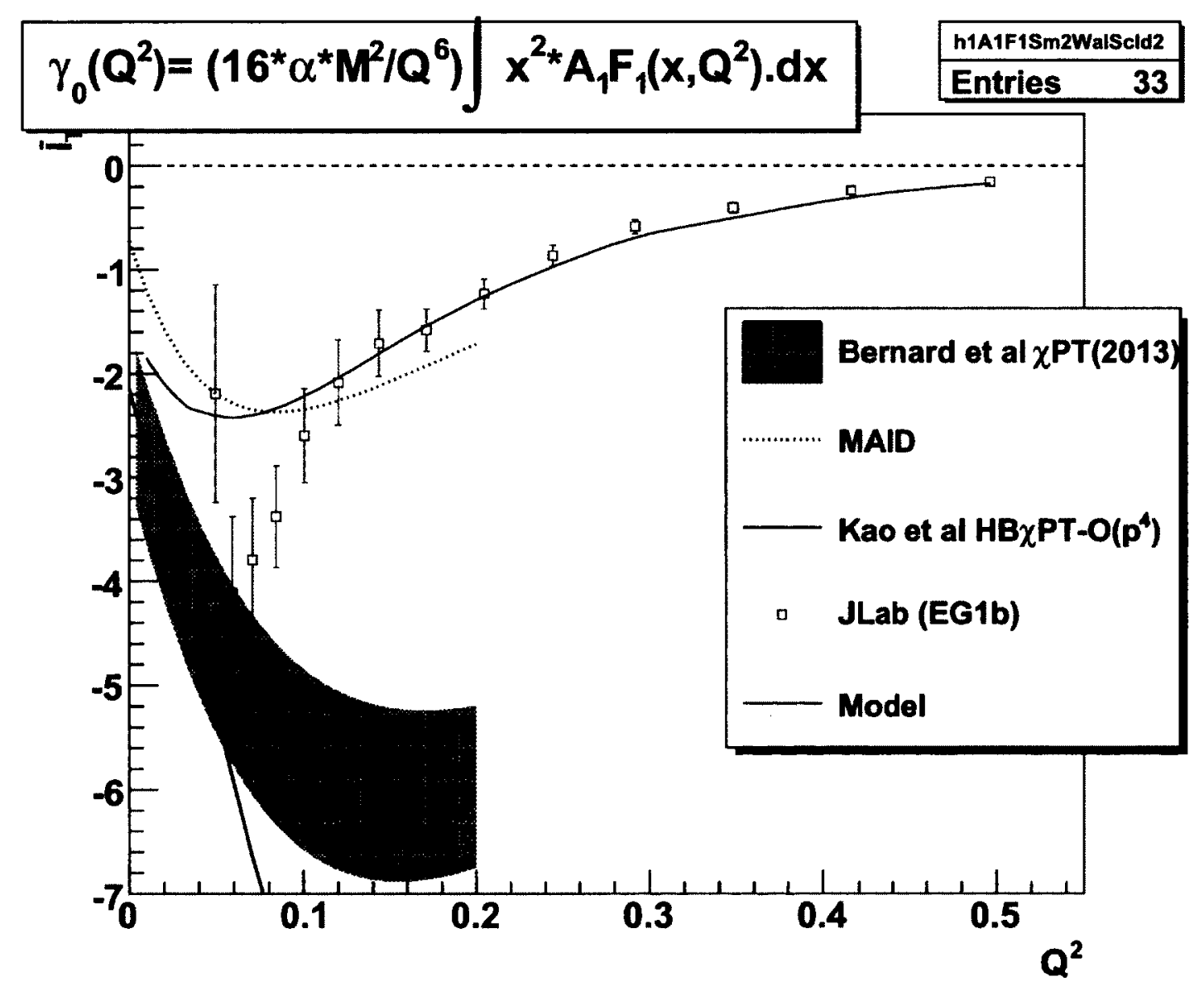

FIG. 9. Some theoretical predictions for $\gamma_{1}^{d}$ together with the recently measured EGlb data. 


\subsection{THEORETICAL TOOLS}

In this section we will take a closer a look at a few common theoretical methods that are used to describe and predict the low $Q^{2}$ behavior/evolution of the structure functions. In addition, some phenomenological models which are also useful in describing the $Q^{2}$ behavior will be reviewed as well.

\subsubsection{CHIRAL PERTURBATION THEORY $(\chi P T)$}

\section{Chiral Symmetry}

QCD is the non-abelian gauge theory of colored quarks and gluons. The complete QCD Lagrangian is:

$$
\mathscr{L}_{Q C D}=-\frac{1}{4 g^{2}} G_{\mu \nu}^{\alpha} G_{\alpha}^{\mu v}+\bar{q} i \gamma^{\mu} D_{\mu} q-\bar{q} \mathscr{M} q
$$

where $\mathrm{G}$ is the gluon field strength, $\mathrm{q}$ is the quark spinor field, and $\mathscr{M}$ is the diagonal quark mass matrix.

For low energy interactions, the quark-gluon degrees of freedom become impractical due to confinement property of QCD. So, effective theories in terms of composite particles such as hadrons as the approximate degrees of freedom are employed to describe such processes and make relevant predictions. To do so, an effective Lagrangian is formed that retains all or most of the symmetries and symmetry breaking patterns as the more fundamental parent theory.

In the effective theory, the quark masses are generally considered to be zero because they are very small (a few MeVs) compared to typical hadronic mass scales (such as proton mass or the mass of the first non-Goldstone resonance $M_{\rho}$ ) and the Lagrangian takes the form

$$
\mathscr{L}_{Q C D}=\mathscr{L}_{Q C D}^{0}+\mathscr{L}_{Q C D}^{\prime}
$$

with

$$
\mathscr{L}_{Q C D}^{\prime}=-\bar{q} \mathscr{M} q
$$

regarded as a perturbation to $\mathscr{L}_{Q C D}^{0}$.

For a massless fermion, chirality is identical to helicity and is a constant of motion. The central idea of the $\chi \mathrm{PT}$ is that the massless left- and right-handed quarks defined by:

$$
q_{L, R}=\frac{1}{2}\left(1 \pm \gamma_{5}\right) q
$$


do not interact with each other so that the theory allows a $U(3)_{L} \times U(3)_{R}$ symmetry. Explicit breaking of this symmetry is then treated as a perturbation. As with any other effective theory, the theory will fail at some point in the energy scale and it has to be superseded by a more fundamental approach.

\section{Chiral Symmetry Breaking and Perturbation Theory}

At very low energy scales, well below the chiral symmetry scale (of the order of $1 \mathrm{GeV}$ ), nucleon dynamics can be described in rigorous terms using $\chi \mathrm{PT}$, because the chiral symmetry of QCD dominates in this region. At low photon virtualities (i.e. small $Q^{2}$ ), the theory can make rigorous predictions on the spin dependent observables by employing a systematic expansion in powers of low momenta and masses of the Goldstone bosons (which are pions when only two "up" and "down" flavors of QCD degrees of freedom are considered) [37].

\section{Baryon Chiral Perturbation Theory}

Over past two decades, a lot of theoretical work has been done on $\chi$ PT calculations. In this section, we highlight some of the calculations that are relevant to the extracted/measured quantities that are covered in this thesis. The calculations are limited to the two flavor case of up and down quarks and they typically examine the $Q^{2}$ evolution of the Compton amplitudes $S_{1}\left(v, Q^{2}\right)$ and $S_{2}\left(v, Q^{2}\right)$ in the low energy and momentum scales. Earlier we saw that the integrals of spin structure functions and the Compton amplitudes are connected through the dispersion relations.

As indicated above the low-energy expansion is made in powers of small momenta (p) and quark (pion) masses, which involves pion loops of the effective theory. Since, the baryon mass in the chiral limit is not negligible, their addition to the theory adds a new scale to it, thus creating a complication - now there is no guarantee that all nextto-leading-order (NLO) corrections at order $p^{4}$ are given completely by one-loop graphs. To get around this added difficulty, two approaches are considered - Heavy Baryon $\chi \mathrm{PT}$ $(\mathrm{HB} \chi \mathrm{PT})$ and Relativistic Baryon $\chi \mathrm{PT}(\mathrm{RB} \chi \mathrm{PT})$. 


\section{Heavy Baryon Chiral Perturbation Theory}

In this approach [36, 39], the baryon masses are considered very large and the chiral expansion is done in powers of the inverse baryon mass or in powers of the pion to nucleon masses $m_{\pi} / M_{N}$, which gives a consistent counting scheme. However, Bernard and others warn that the expansion may not converge very fast. In line with that warning, a significant $Q^{2}$-variation was observed in the extended GDH sum when the NLO $\left(\mathscr{O}\left(p^{4}\right)\right.$ order was calculated in $\chi \mathrm{PT}$.

\section{$\chi$ PT with Resonance and Vector Meson Contribution}

The chiral models discussed so far include only the pion-nucleon contributions, with no resonance considerations which are expected to have significant contributions to the Compton amplitudes, especially from the $\Delta(1232)$ resonance. The best approach to adding the $\Delta$ contribution would be to include the resonance as a new dynamical degree of freedom in the effective Lagrangian, but such an effective theory of 3-body pion-nucleon-delta system has not been tried or published yet. Rather, as a way around, a systematic addition of the $\Delta$ contribution in the heavy baryon framework has been attempted, with the nucleondelta mass difference treated as an additional parameter. The $\Delta$ resonance contribution is estimated by calculating relativistic Born cross-sections that are functions of a number of "not-well-known" experimental parameters. Due to the uncertainties in these parameters, the model predictions are in the form of a band of values. Some authors [40] have also added vector meson contributions.

One possibility in getting around the resonance contributions in order to make meaningful predictions over a wider range of distance scales (thus providing good tests of the theoretical model) is to calculate and examine quantities involving the difference between proton and neutron observables (such as the Bjorken sum rule). In such a difference, the resonance contribution mostly cancels out leaving a more reliable $\chi$ PT prediction that has a reduced $Q^{2}$ dependence [ 41 ].

\subsubsection{METHOD OF OPERATOR PRODUCT EXPANSION}

OPE provides direct predictions for moments of structure functions in the form of sum rules. It was introduced by Wilson [42] as a way to evaluate the non-perturbative part of QCD calculations. The method is called OPE because it allows evaluation of product of two operators (representing, for example, some observables such as the electromagnetic 
currents $\left.J_{\mu}(\xi) J_{v}(0)\right)$ in the asymptotic limit of spatial vectors becoming infinitesimal (by expanding it into a series of terms with Wilson coefficients containing pQCD calculable spatial dependence, and only a few terms significant for large enough $Q^{2}$ (equivalently small enough spatial 4-vector) compared to the relevant mass scale $\Lambda_{Q C D}^{2}$ )).

$$
\lim _{\xi \rightarrow 0} \mathscr{O}_{a}(\xi) \mathscr{O}_{b}(0)=\sum_{i} C_{a b k}(\xi) \mathscr{O}_{i}(0)
$$

The remaining factor $\mathscr{O}_{i}(0)$ of each expansion term is a quark-gluon operator of dimension $\mathrm{d}$ and spin $\mathrm{n}$, representing the fundamental fields in QCD. The concept of twist $\tau=d-n$ is introduced for the contribution of any operator to $\mathscr{L}_{\mu v} W^{\mu v}$ i.e. the differential cross section is of the order:

$$
\omega^{n}\left(\frac{M}{Q}\right)^{\tau-2}
$$

The lowest possible twist is "twist-2". At large $Q^{2}$, the leading twist term dominates because the higher twists are suppressed by increasing powers of $\mathrm{M} / \mathrm{Q}$, and obviously, one can expect the higher terms to be important in the low $Q^{2}$ region. The reliable parts of the parton model map onto the leading twist part of the OPE, while the twist- 3 and higher arise from quark-gluon interactions and non-zero quark mass effects. By connecting the matrix element for virtual Compton scattering to the hadronic tensor $W_{\mu v}$ through the Optical theorem, the twist expansion leads to an infinite set of sum rules for the structure functions, both polarized and unpolarized. For example, disregarding contributions beyond twist-3,

$$
\begin{array}{cc}
\int_{0}^{1} x^{n-1} g_{1}\left(x, Q^{2}\right) d x=\frac{1}{2} a_{n-1} & n=1,3,5, \ldots \\
\int_{0}^{1} x^{n-1} g_{2}\left(x, Q^{2}\right) d x=\frac{n-1}{2 n}\left(d_{n-1}-a_{n-1}\right) & n=1,3,5, \ldots
\end{array}
$$

where $a_{n-1}$ and $d_{n-1}$ are the twist-2 and twist- 3 matrix elements of the renormalized quark and gluon operators respectively. Notice that only odd values of $\mathbf{n}$ contribute due to the symmetry properties of the structure functions under the charge conjugation.

By measuring the spin structure functions at moderate to high $Q^{2}$ over the entire $\mathrm{x}$ range, these sum rules allow extraction of higher twist matrix elements arising from parton interactions. Confinement arises from such interactions, so such higher twist measurements offer tools to examine one of the fundamental properties of QCD. One can expect higher twist effects becoming more and more significant until at some point the whole twist expansion approach breaks down. 


\section{Higher Twists Effects in $g_{1}$}

The generalization of equation 75 to include all orders of higher twist is:

$$
\Gamma_{1}\left(Q^{2}\right)=\sum_{\tau=2,4, . .} \frac{\mu_{\tau}\left(Q^{2}\right)}{Q^{\tau-2}}
$$

with $\Gamma_{1}\left(Q^{2}\right)=\int d x g_{1}\left(x, Q^{2}\right)$. Notice that the OPE sums over all possible states including the elastic contribution.

One can gain access to the higher twist contributions to $\Gamma_{1}$ by subtracting the leading ( $\tau=2)$ twist $\mu_{2}\left(Q^{2}\right)$ term from the experimentally measured value of the first moment. Up to $\mathscr{O}\left(\alpha_{s}^{3}\right)$ in the strong coupling constant for three quark flavors, the result for the leading twist terms of $\Gamma_{1}$ is given by:

$$
\begin{aligned}
\mu_{2}^{p(n)} & =\left[1-\left(\frac{\alpha_{s}}{\pi}\right)-3.58\left(\frac{\alpha_{s}}{\pi}\right)^{2}-20.22\left(\frac{\alpha_{s}}{\pi}\right)^{3}\right]\left( \pm \frac{1}{12} g_{A}+\frac{1}{36} a_{8}\right) \\
& +\left[1-\frac{1}{3}\left(\frac{\alpha_{s}}{\pi}\right)-0.55\left(\frac{\alpha_{s}}{\pi}\right)^{2}-4.45\left(\frac{\alpha_{s}}{\pi}\right)^{3}\right] \frac{1}{9} \Delta \Sigma \\
& =\left( \pm \frac{1}{12} g_{A}+\frac{1}{36} a_{8}\right)+\frac{1}{9} \Delta \Sigma+\mathscr{O}\left(\alpha_{s}\left(Q^{2}\right)\right)
\end{aligned}
$$

with,

- \pm : for proton and neutron respectively

- $\alpha_{s}$ : the strong coupling constant

- $g_{A}$ : the non-singlet triplet axial charge measured precisely from neutron $\beta$ decay

- $a_{8}$ : the octet axial charge, extracted from weak hyperon decays assuming SU(3) symmetry.

- $\Delta \Sigma$ : the singlet axial current. In the parton model, it is the amount of spin carried by quarks, which has been extracted from global analysis of world DIS data.

- $\mathscr{O}\left(\alpha_{s}\right): Q^{2}$ evolution due to QCD radiative effects (calculable from PQCD)

The first non-leading order contribution is the $\frac{1}{Q^{2}}$ term:

$$
\mu_{4}\left(Q^{2}\right)=\frac{1}{9} M^{2}\left[a_{2}\left(Q^{2}\right)+4 d_{2}\left(Q^{2}\right)-4 f_{2}\left(Q^{2}\right)\right]
$$


where $a_{2}$, the second moment of $g_{1}$ arises from the target mass correction and is pure twist-2. $d_{2}$, as revealed above, is primarily twist-3 $f_{2}$ represents the only pure twist-4 contribution to $\mu_{4}$. The twist- 3 and -4 operators contain all the interaction information and collectively describe the response of the color electric and magnetic fields to the presence of the nucleon spin. This behaviour is encorporated in the color electric and magnetic polarizabilities $\chi_{E}$ and $\chi_{B}$, which are related with the matrix elements as follows:

$$
\begin{aligned}
& \chi_{E}=\frac{2}{3}\left(2 d_{2}+f_{2}\right) \\
& \chi_{B}=\frac{1}{3}\left(4 d_{2}-f_{2}\right)
\end{aligned}
$$

The difference between the first moments of the proton and neutron $g_{1}$ (using Eq. 78) gives rise to the well known Bjorken sum rule as $Q^{2} \rightarrow \infty$ :

$$
\Gamma_{1}^{p}\left(Q^{2}\right)-\Gamma_{1}^{n}\left(Q^{2}\right)=\frac{1}{6} g_{A}+\mathscr{O}\left(\alpha_{s}\left(Q^{2}\right)\right)+\mathscr{O}\left(1 / Q^{2}\right)
$$

Bjorken first derived this sum rule using the current algebra method, so it provides a fundamental test of the structure of QCD. With the PQCD corrections included, the sum rule has been tested and verified to the level of $10 \%$.

\subsubsection{PHENOMENOLOGICAL MODEL PREDICTIONS}

There exist several phenomenological models that parameterize existing world data. In this subsection, we will examine some of those which are used for predictions and analysis of the observables in the kinematic region covered by our experimental data.

\section{MAID}

The Mainz-Dubna (MAID) parameterization is a unitary isobar model relying on phenomenological fits to the world experimental data in the form of cross sections and polarization asymmetries from pion photo- and electro-production in the resonance region (traditionally defined as the range from the pion production threshold up to $\mathrm{W}=2 \mathrm{GeV}$ and photon virtualities $Q^{2}<5 \mathrm{GeV}^{2}$ ). The model is used for partial wave analysis of pion photo- and electro-production data in the resonance region, with predictions possible for multipoles, amplitudes, cross sections and polarization observables [43]. The model 
contains both the non-resonant background and many resonance terms (13 of the fourstar $^{6}$ resonances with masses below $2 \mathrm{GeV}$ ), unitarized according to the prescription of $\mathrm{K}$-matrix theory and using appropriately unitarized Breit-Wigner functions to construct the various resonance production channels [38]. For example, the contribution of a particular resonance to the transverse cross section is given by:

$$
\sigma_{\frac{1}{2}\left(\frac{3}{2}\right)}=\frac{4 M}{W_{0} \Gamma_{0}} A_{\frac{1}{2}\left(\frac{3}{2}\right)}^{2} B\left(v, Q^{2}\right)
$$

where, $B\left(v, Q^{2}\right)$ is the Breit-Wigner distribution function generalized to electroproduction, $\mathrm{M}$ is the nucleon mass, $W_{0}$ is the resonance mass, $\Gamma_{0}$ is the resonance width and $A_{\frac{3}{2}}^{2}$ is the corresponding helicity dependent photo-coupling amplitude. In addition to these resonant terms, contribution terms for the non-resonant background as well as t-channel vector meson exchanges are also included [15][43].

The predictions from this model are in good agreement with both polarized and unpolarized data on pion photo- and electro-production from the nucleon. The model also agrees with the GDH sum rule on the proton (at the real photon point) but does not predict the rule for the neutron at low $Q^{2}$. The discrepancy between the data and the neutron prediction could be due either to the fact that final state interactions for pion production from "effective-neutron" nuclear targets (deuteron or ${ }^{3} \mathrm{He}$ ) are neglected (not well-accounted for) or two-pion contribution are larger than assumed or possible modification of multipole expansion due to the nuclear binding effects [19].

\section{Burkert and Ioffe}

Burkert and Ioffe proposed a phenomenological model [44][45] to describe the $Q^{2}$ evolution of sum rules for real and virtual photon scattering off nucleons. This model is built on an earlier proposed vector meson dominance model for the GDH integral by Anselmino, Ioffe and Leader [31].

The older model interpolated the measured high $Q^{2}$ (asymptotic) value of the integral down to $Q^{2}=0$ point of the real-photon GDH sum rule, by using a two parameter function

\footnotetext{
${ }^{6}$ The star system is used by the PDG to indicate the strength of the evidence for a given resonance.

- **** - Existence convincingly established with properties at least fairly well-explored [21]

- *** - Existence very likely but further confirmations required.

- ** - Evidence of existence only fair.

- * Evidence of existence poor.
} 
as follows:

$$
I_{1}^{V D M}\left(Q^{2}\right)=2 M^{2}\left[\frac{1}{Q^{2}+\mu^{2}}-c \frac{\mu^{2}}{Q^{2}+\mu^{2}}\right] \Gamma_{1}(\infty)
$$

where the mass parameter $\mu$ (taken at $\rho$ or $\omega$ mass) sets the scale of the $Q^{2}$ evolution and the other parameter $\mathrm{c}$ is chosen such that the integral (I) at $Q^{2}=0$ coincides with the GDH sum rule. The authors of the model contended that the two terms of the interpolation function represented two dominant diagrams in the VDM picture of the photon-nucleon interaction.

The older model, which ignored the large contribution of the low $\mathrm{W}$ resonant states, was improved by Burkert and Ioffe by explicitly adding the contributions for individual resonances (upto $\mathrm{W}=1.8 \mathrm{GeV}$ ) extracted from pion electroproduction data. Now, in the refined model, the GDH integral has two parts - one given by eq. 85 and another being that for the resonance contributions as follows:

$$
I_{1}\left(Q^{2}\right)=I_{1}^{V D M}\left(Q^{2}\right)+I_{1}^{R e s}\left(Q^{2}\right)
$$

where the parameter $\mathrm{c}$ in for the first term as represented by eq. 85 is given by:

$$
c=1+\frac{1}{2} \frac{\mu}{M} \frac{1}{\Gamma_{1}(\infty)}\left[\frac{\kappa^{2}}{4}+I_{1}^{\text {Res }}(0)\right]
$$

with $M, \kappa$ being the mass and the magnetic moment of the nucleon. And, the second term is approximated by the amplitudes for the pion electroproduction $\left(\gamma^{*} N \rightarrow N^{*} \rightarrow N \pi\right)$ data which are reasonably well known from phase shift analysis.

This model predicts that $\Gamma_{1}\left(Q^{2}\right)$ changes sign at about $Q^{2}=0.4 \mathrm{GeV}^{2}$, which is attributed to a large negative contribution of $\Delta(1232)$-resonance.

\section{Soffer and Teryaev}

Soffer and Teryaev proposed [46] another model suggesting that the strong $Q^{2}$-dependence of the GDH integral $I_{1}\left(Q^{2}\right)$ should be studied in combination with the $g_{2}$-counterpart i.e. $I_{2}\left(Q^{2}\right)=\frac{2 M^{2}}{Q^{2}} \int g_{2}\left(x, Q^{2}\right) d x$ (which is also known as Schwinger integral for the namesake sum rule). Assuming that the evolution of $I_{T}=I_{1+2}\left(=\frac{2 M^{2}}{Q^{2}} \int\left(g_{1}+g_{2}\right)\left(x, Q^{2}\right) d x\right)$ from DIS to low $Q^{2}$ is smooth, the authors first express the GDH integral as $I_{1}=I_{1+2}-I_{2}$. It is also assumed that the Burkhard-Cottingham (BC) sum rule (i.e. $\int_{0}^{1} g_{2}\left(x, Q^{2}\right) d x=0$ ) 
is valid (with the elastic contribution included in the integral) generating the strong $Q^{2}$ dependence of $I_{2}$. The constraint of $\mathrm{BC}$ sum rule implies that the inelastic contribution to $I_{2}$ (all of these integrals are defined for the interval $x[0,1]$ ) is given by

$$
I_{2}\left(Q^{2}\right)=\frac{\mu G_{M}\left(Q^{2}\right)}{4}\left[\frac{\mu G_{M}\left(Q^{2}\right)-G_{E}\left(Q^{2}\right)}{1+\frac{Q^{2}}{4 M^{2}}}\right]
$$

which gives $I_{2}(0)=\frac{\kappa^{2}+e x}{4}$ with $e$ being the nucleon charge. For the proton a smooth interpolation is made between asymptotic limits for $I_{T}$ for which the lower limit is provided by the combination of "GDH sum rule" ( $=g_{1}$ integral at photon point) and "Schwinger sum rule" (=g $g_{2}$ integral at $Q^{2}=0$ ) and upper limit is provided by the fact that $Q^{2} \rightarrow \infty, I_{T} \rightarrow$ $\frac{2 M^{2}}{Q^{2}} \Gamma_{1}(x)$. And, the large $Q^{2}$ behavior of the interpolation is set to match the existing world data. A similar procedure is implemented for the neutron, however, the interpolating function is used to represent the isovector difference $I_{T}^{p-n}\left(Q^{2}\right)$.

Most recently, Pasechnik, Soffer and Teryaev [47] have improved their previous QCDinspired model for the $Q^{2}$ evolution of the extended GDH integral by adding the latest results extracted from Jefferson lab data, particularly the results on the higher order radiative and higher-twist power corrections to the first moment $\Gamma_{1}^{p}\left(Q^{2}\right)$ of the proton's $g_{1}$ structure function and the sum $\Gamma_{1}^{p-n}\left(Q^{2}\right)$ of the Bjorken sum rule.

\subsubsection{LATTICE QCD}

Lattice QCD is a Lattice Gauge Theory which is defined on a spacetime that is discretized into a lattice. Gauge theories describing the interactions of elementary particles (such as for QED, QCD) can sometimes be solved perturbatively. When one has to use a non-perturbative apporoach, then the related calculations become computationally intractable if this is done in the continuous spacetime, because that would require evaluating an infinite-dimensional path integral. But, in a discrete spacetime grid of finite size, the path integral becomes finite dimensional and can be evaluated using powerful computers by implementing stochastic simulation techniques such as Monte Carlo methods. The exact continuum gauge theory is then recovered by extrapolating the LQCD results to the limiting case of infinitely large lattice and infinitesimally close grid points [48].

Lattice QCD provides a framework for a non-perturbative approach to solving QCD in order to calculate the structure and properties of strongly interacting particles and processes. Being non-perturbative in nature, the theory, in principle, is useful in making predictions at all kinematic scales. However, the calculations are numerically extremely 
intensive and computationally very costly warranting the use of very powerful supercomputers or big LQCD dedicated clusters of powerful computers. The method becomes even more intensive and costlier computationally as one uses larger lattices and smaller lattice spacings to ensure reliable predictions for the desired observables. To reduce the computational burden, certain approximations (e.g., quenched field approximation used in the early lattice calculations, with the quark fields treated as non-dynamic "frozen" variables) are used [49].

The LQCD is a rapidly developing field with significant progress made in algorithms, together with a steady increase in the computational technologies and capabilities leading to better calculations and enabling theorists to make a number of predictions that match well with the experimental data. For example, the proton mass has been calculated within the 2 percent error of the well known value [50]. It is hoped that the lattice calculations will bridge the gap in the intermediate $Q^{2}$ regime, where no other method (neither PQCD, nor OPE or $\chi \mathrm{PT}$ ) is precise enough to make predictions. A strong connection between lattice and $\chi \mathrm{PT}$ calculations developed recently. One approach has been to use the $\chi \mathrm{PT}$ predictions to make LQCD extrapolations, thus, tying the LQCD results with $\chi$ PT and making the experimental verification of $\chi \mathrm{PT}$ calculations essential to the test of LQCD results $[51,52]$.

\subsection{THE DEUTERON}

The structure functions, their moments and polarizabilities defined for the nucleons are also valid for the deuteron and the work of this thesis focuses solely on the deuteron results. So, it is worthwhile to have a closer look at this particular nucleus.

The deuteron is the bare nucleus of the heavier and less abundant isotope ${ }^{7}$ of hydrogen known as deuterium. It is made up of two nucleons - a proton and a neutron bound together with nuclear forces amounting to a binding energy of about $2.22 \mathrm{MeV}$ [53]. It has a mass of 1875.6 - nearly double the mass of a proton. It is the only stable bound system of two nucleons found in nature.

The deuteron in its ground state is in an isospin singlet state which is antisymmetric

\footnotetext{
${ }^{7}$ The natural abundance relative to the ordinary hydrogen is about one atom in 6,700 of hydrogen [53].
} 
under the exchange of the proton and the neutron ${ }^{8}$. In addition to the isospin, the nucleons also have spins and spatial distributions. The symmetry for the latter is known as parity (denoted by $P$ ) which dictates how the wave functions change under the exchange of the locations of the constituent nucleons (the symmetric and antisymmetric exchanges are said to have even or positive and odd or negative parities respectively and are fully determined by the total orbital angular momentum $L$ as given by $P=(-1)^{L}$ ).

Since the deuteron (wave function) is antisymmetric in the isospin representation, it must be symmetric under the double exchange of constituent nucleon spins and locations. This means that the deuteron can either be in a state in which it is symmetric under both spin and parity or in a state in which both are antisymmetric. In the first case, the deuteron is a spin triplet with the total spin of 1 and even orbital angular momentum 1 (to ensure even parity). The lowest possible energy state in this category has $s=1$ and $l=0$. In the second possible state, the deuteron is a singlet with the lowest possible energy state having $s=0$ and $l=1$. Since the $s=1$ gives a stronger nuclear attraction, the first state turns out to be the deuteron ground state [53]. Therefore, the deuteron has spin +1 ("triplet") and is thus a boson. The fact that the deuteron ground state is the $S$-state with $S=1, L=0$ (even parity) (and thus $\mathrm{J}=1$ ) is only an approximation, and in reality, the $\mathrm{D}$-state with $\mathrm{L}=2$ is also possible and contributes a small part to the ground state. The fact that the precisely measured deuteron magnetic dipole moment $\left(\mu_{d}=0.8574\right)$ is slightly different from the total of the moments of proton and neutron $\left(\mu_{p}+\mu_{n}=0.8797\right)$ indicates that higher orbital momentum states are also contributing to the deuteron wave function. The electric quadrupole moment ${ }^{9}$ for deuteron is also measured to be non-zero $\left(=0.2859 e \cdot \mathrm{fm}^{2}[53]\right)$, indicating that the charge distribution in the deuteron cannot solely be a spherically symmetric S-state, rather it must be a quantum mixture of $S$ and $D$ states with $L=0$ and $L=1$ respectively. The $S$-state, which

\footnotetext{
${ }^{8}$ Totally analogous to the ordinary spin, isospin is a SU(2) symmetry. Proton and neutron are considered as two isospin types or states of the same object commonly known as nucleon. In other words, the two possible isospin states of a nucleon are said to form an isospin doublet, with the isospin "up" and "down" states of the doublet being identified as proton and neutron respectively.

In contrast to the doublet for a single nucleon, a pair of nucleons can exist in any of the following four possible isospin states - one being the antisymmetric isospin singlet $\frac{1}{\sqrt{2}}(|\uparrow \downarrow>-| \downarrow \uparrow>)$ with a total of 0 isospin (i.e., neither "up" nor "down") and the other three being the symmetric isospin "triplet" states $\left(|\uparrow \uparrow\rangle, \quad \frac{1}{\sqrt{2}}(|\uparrow \downarrow\rangle+|\downarrow \uparrow\rangle), \quad|\downarrow\rangle\right)$, with total isospins of $(1,0,-1)$ respectively. The antisymmetric singlet state is identified as the ordinary deuteron in its stable ground state, whereas the other three symmetric states are identified with three very highly unstable objects - a nucleus of two protons, a highly excited state of a deuterium nucleus and a nucleus with two neutrons respectively [53].

${ }^{9}$ The electric dipole moment for deuteron is zero as it is for all nuclei.
} 
has the spins of both nucleons aligned along the deuteron spin, can be expressed as:

$$
\left|J=1, J_{z}=1\right\rangle=\left|L=0, L_{z}=0\right\rangle\left|S=1, S_{z}=1\right\rangle
$$

whereas, the D-state, in which z-projections of the nucleon spins are not always aligned with the total angular momentum can be written as:

$$
\begin{aligned}
\left|J=1, J_{z}=1\right\rangle & =\sqrt{\frac{1}{10}}\left|L=2, L_{z}=0\right\rangle\left|S=1, S_{z}=1\right\rangle \\
& -\sqrt{\frac{3}{10}}\left|L=2, L_{z}=1\right\rangle\left|S=1, S_{z}=0\right\rangle \\
& +\sqrt{\frac{3}{5}}\left|L=2, L_{z}=2\right\rangle\left|S=1, S_{z}=-1\right\rangle
\end{aligned}
$$

The probability of finding the deuteron in the $D$-state is $\omega_{D} \approx 0.056$ [35]. Therefore, from eq.90, the likelihood of finding a nucleon in the spin-down state is $\frac{3}{4} \omega_{D}$. Using this fact and ignoring other nuclear effects (to be discussed later), we get the following deuteron cross sections (normalized as "per nucleon") in terms of those of the nucleons:

$$
\begin{aligned}
& \sigma_{d}^{\uparrow \downarrow}=\left(1-\frac{3}{4} \omega_{D}\right) \sigma_{N}^{\uparrow \downarrow}+\frac{3}{4} \omega_{D} \sigma_{N}^{\uparrow \uparrow} \\
& \sigma_{d}^{\uparrow \uparrow}=\left(1-\frac{3}{4} \omega_{D}\right) \sigma_{N}^{\uparrow \uparrow}+\frac{3}{4} \omega_{D} \sigma_{N}^{\uparrow \downarrow}
\end{aligned}
$$

with $\sigma_{N}=\left(\sigma_{p}+\sigma_{n}\right) / 2$ and the two arrows indicate the spin directions of the beam electron and the target relative to the beam direction. If these two equations are substituted into the basic definition of the virtual photon asymmetry $A_{1}=\left(\sigma_{\frac{1}{2}}^{T}-\sigma_{\frac{3}{2}}^{T}\right) /\left(\sigma_{\frac{1}{2}}^{T}+\sigma_{\frac{3}{2}}^{T}\right)$, one gets,

$$
A_{1}^{d}=\left(1-\frac{3}{2} \omega_{D}\right)\left[\frac{\sigma_{p}^{T} A_{1}^{p}+\sigma_{n}^{T} A_{1}^{n}}{\sigma_{p}^{T}+\sigma_{n}^{T}}\right]
$$

where the subscript " $T$ " in $\sigma$ indicates the transverse polarization of the exchanged virtual photon. By using the relations between the virtual photoabsorption cross sections and the structure functions as discussed in section 2.1.3, one gets the following relation:

$$
g_{1}^{d}\left(x, Q^{2}\right)=\left(1-\frac{3}{2} \omega_{D}\right)\left[\frac{g_{1}^{p}\left(x, Q^{2}\right)+g_{1}^{n}\left(x, Q^{2}\right)}{2}\right]
$$

with the factor $1 / 2$ introduced to express $g_{1}^{d}$ as "per nucleon" value (the factor in front represents the ratio of the nucleon polarization $P_{n}$ to the deuteron polarization $P_{d}$ ). 


\subsubsection{EXTRACTION OF NEUTRON INFORMATION FROM A DEUTERON TAR- GET}

One of the objectives of having deuteron data is that it can be used in combination with similar data on the proton target to extract the corresponding neutron information (this is important because a free neutron target is impractical). Once the deuteron and proton data is available, to a first order approximation, simply subtracting proton results from deuteron ones would be expected to give neutron information. But, a nucleus is not simply a collection of the two free neutrons, but it is more complex than that with various nuclear medium effects (such as Fermi motion, deuteron D-state correction, Offshell effects, EMC effect) These effects must be understood and properly accounted for to extract neutron information from the deuteron. In addition, in order to have confidence in the extracted neutron results, it is also important to have data on other nuclei such as ${ }^{3} \mathrm{He}$ targets.

In the resonance region and at large $x>0.5$, Fermi motion and the depolarizing effect of the D-wave are considered the most important nuclear effects. (the latter already considered in Eqs. (93) and (94)).

\section{Fermi Motion}

As in any other nucleus, the bound nucleons in a deuteron are in a constant random motion of quantum origin called Fermi-motion. Due to this motion, an incident lepton does not see a nucleon at rest but with some momentum, resulting in systematic kinematic shifts and smearing which causes the various nucleon resonances to show up at slightly shifted places and their peaks/widths to suffer some Doppler broadening. Due to this fact, if one attempts to extract the neutron structure functions by subtracting the proton ones from those of the deuteron, a 'true' maximum in the proton structure function may result in a 'false' minimum in the those of the neutron and vice versa, even if we assumed similar behavior for the two in the beginning. For this reason, the Fermi smearing becomes an important effect (particularly important at high $\mathrm{x}$ and in the resonance region) to be considered while extracting correct neutron information from the deuteron and proton data.

\section{Folding Algorithm to model Deuteron}

Recently Kahn et al [54] suggested a new convolution method to extract neutron structure functions from nuclear data. This method uses iterative technique to take these effects into 
account and extract the neutron information. The method convolutes proton and neutron structure functions (SFs) to model the deuteron and relies on the knowledge of the proton and deuteron to iteratively extract the neutron SFs. The process starts with a predefined input function for the neutron which is then evolved iteratively until the function becomes stable. In the current form, the method considers only the two major sources of corrections - the Fermi motion and the D-state of the deuteron and ignores other nuclear effects. Still, the method is capable of including other corrections as well. It has been tried and tested well on the unpolarized structure functions which show no sign change. The spin structure function $g_{1}$, however, has several sign changes in the resonance region, causing the iterative method to fail in some kinematic regions when one uses data with errors for the proton and deuteron. But this method can be made reliable by using parameterizations of the structure functions, instead, as was done successfully in the EG1b data analysis [35].

In our analysis, we did not extract information on the neutron but we did use this convolution method to model the deuteron. 


\section{CHAPTER 3}

\section{EXPERIMENTAL TOOLS AND SET UP}

The EG4 experiment was performed in the experimental Hall-B of the Thomas Jefferson National Accelerator Facility (TJNAF, also known as Jefferson lab or simply JLab ) in Newport News, Virginia. The experiment ran from February to May in 2006. Longitudinally polarized $(\approx 85 \%$ polarization) electron beams from the CEBAF linear accelerator (with beam energies 1.0, 1.3, 1.5, 2.0, 2.3 and 3.0 at different times) were scattered off longitudinally polarized solid ammonia targets (polarizations up to $\approx 90 \%$ and $\approx 45 \%$ for $\mathrm{NH}_{3}$ and $\mathrm{ND}_{3}$ respectively). The particles scattered or produced in this process were detected using the unique CLAS (CEBAF Large Acceptance Spectrometer) detector and with the help of the Hall-B triggering and data-acquisition (DAQ) system, the detector signals (that passed the criteria for viable scattering events) were sorted out and recorded on tape silos for later off-line analysis. In the following sections, all these experimental components are introduced and the way they work is described.

\subsection{CEBAF LINEAR ACCELERATOR}

As with all other electron or photon scattering experiments carried out in the experimental halls in Jefferson Lab, the polarized electron beam for EG4 was provided by the Continuous Electron Beam Accelerator Facility (CEBAF) (Fig. 10) [55]. CEBAF is capable of providing beams of up to $6 \mathrm{GeV}^{1}$ with energy spread $\Delta E / E \sim 10^{-4}$ and currents up to $300 \mu \mathrm{A}$, with a $1497 \mathrm{MHz}$ pulse structure, which are then sent to the halls in a round robin fashion so that the effective pulse frequency in each hall is $1497 / 3(=499 \mathrm{MHz})$. The beam polarization is up to $90 \%$ and the charge per bunch is up to $3 \mathrm{pC}$ [56]. The CEBAF consists mainly of three elements: an injector that produces a $45 \mathrm{MeV}$ polarized beam, two linacs (north and south) each boosting the energy of an electron by upto $600 \mathrm{MeV}$ in one pass, and two recirculating arcs to steer the beams from one linac to another for up to

\footnotetext{
${ }^{1}$ Currently, JLab is undergoing an upgrade that will enable it to generate and work with beams up to 12 $\mathrm{GeV}$.
} 
five passes (controlled by by beam switch-yard operator) before the beam delivery to the halls.

\section{HOW CEBAF WORKS}

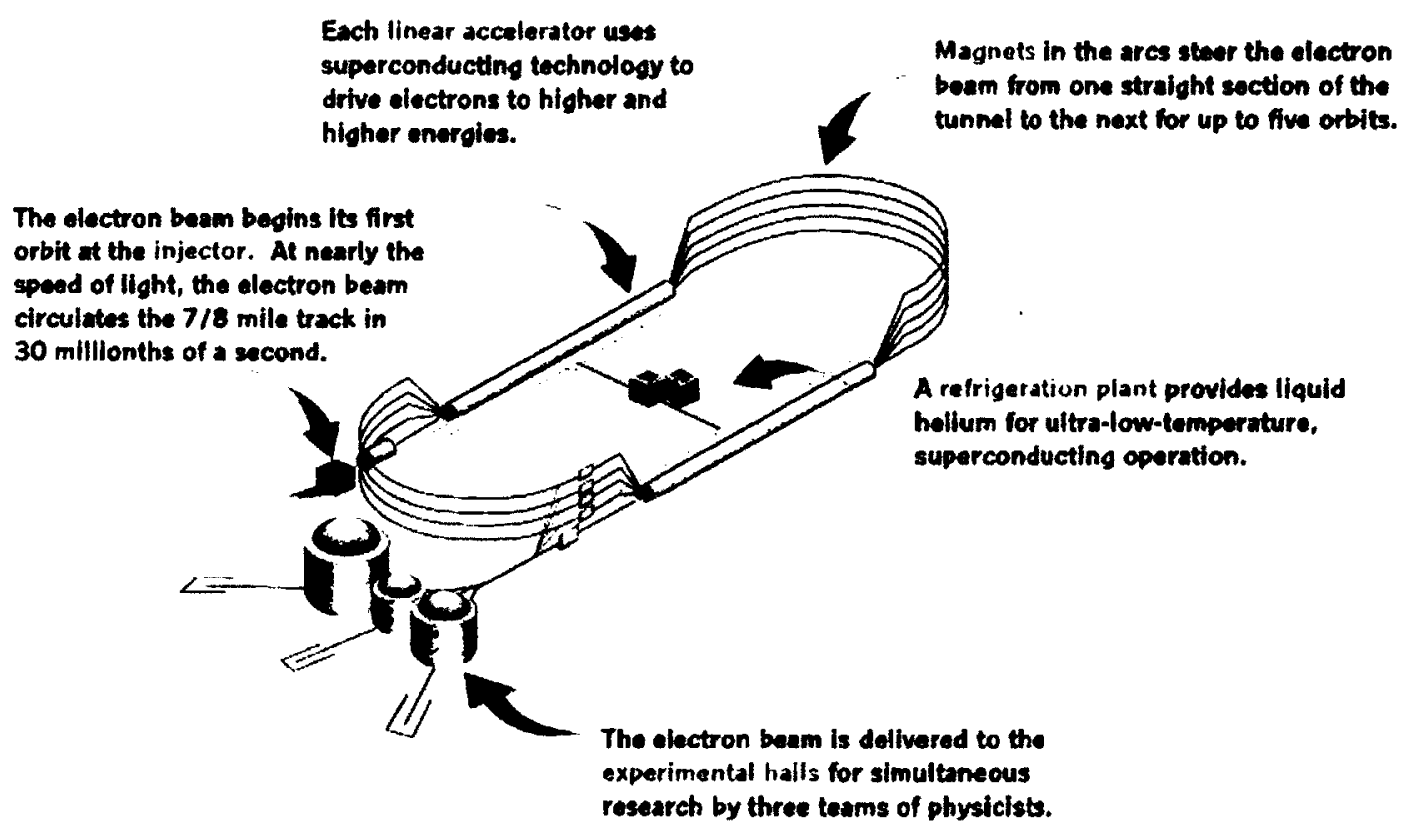

FIG. 10. CEBAF accelerator at Jefferson Lab [55].

The injector uses a state-of-the-art room-temperature electron gun system with a strained GaAs photocathode, which is capable of delivering high polarization (up to $90 \%$ ), high current $(\sim 200 \mu \mathrm{A})$, continuous [57] wave beams into the accelerator and eventually to the three end stations (Halls $\mathrm{A}, \mathrm{B}$ and $\mathrm{C}$ ), while maintaining a low current, high polarization beam to Hall $B$ [58]. In the injector, beams of circularly polarized light from a unique system of three diode lasers - each pulsed with the frequency of $499 \mathrm{MHz}$ (the third subharmonic of the accelerating RF i.e., $1497 \mathrm{MHz}$ ) - illuminates the cathode under ultra-high vacuum level ( $\sim 10^{-12}$ Torr) [59]. That causes the excitation of electrons from the top (spin-biased) valence band to the conduction band, thus emitting a $1497 \mathrm{MHz}$ pulse-train of low energy linearly polarized electrons. The direction of the polarization can be flipped by using a voltage driven Pockel cell at about $30 \mathrm{~Hz}$ and occasionally reversed, which flips 
the polarization of the laser light and consequently flipping the photo-electron polarization. (During most polarized target experiments, data is collected with a "half-wave-plate" (HWP) inserted and removed semi-regularly so as not to have a polarization dependent bias in the data.) The electrons are first accelerated to $100 \mathrm{keV}$ by the $100 \mathrm{kV}$ field in the gun before passing them through a pre-buncher cavity and two circular apertures, where the beam is pared down to improve the distorted shape and size of the bunches (due to space charge effects). Thereafter, an optical chopper splits the beam into $499 \mathrm{MHz}$ bunches designated for the three halls, and sends them through a buncher cavity, followed by a capture section made of a five-cell cavity, which further accelerates the beams to $500 \mathrm{keV}$ while controlling the beam bunch length and energy spread with the unwanted electrons steered away to a beam dump. Next, the beam passes through two superconducting (SRF) cavities to get further bunched and accelerated to $5 \mathrm{MeV}$. At the end, two cryomodules - each with 8 SRF cavities - boosts the beams to the final injector energy of $45 \mathrm{MeV}$ and then injects it into the north Linac by bending it with a chicane magnet. The bending produces synchroton light with intensity proportional to the beam current, which is exploited by a Synchroton Light Monitor (SLM) to monitor the relative beam current $[60,61]$.

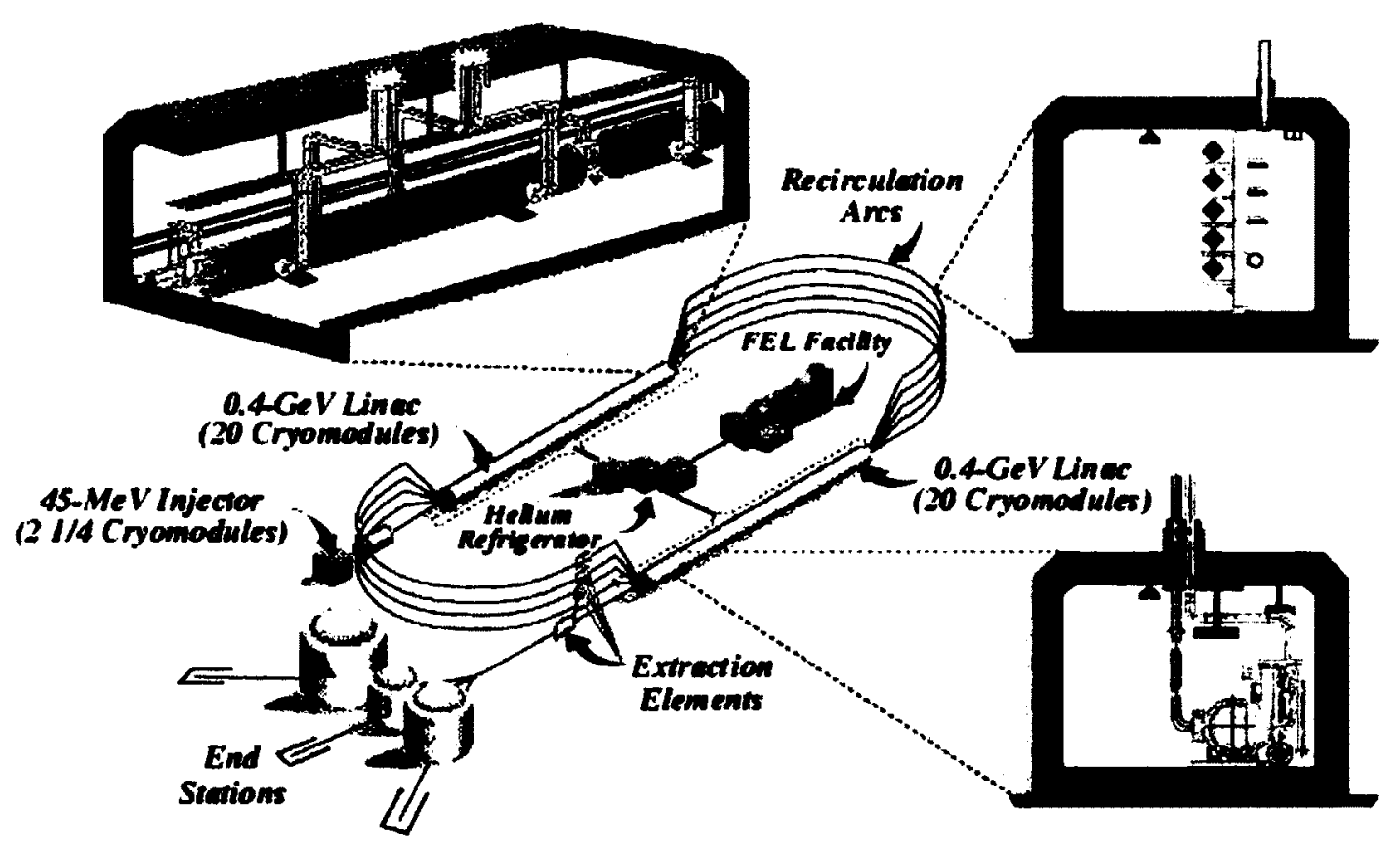

FIG. 11. CEBAF accelerator and some components

The $45 \mathrm{MeV}$ beam injected into the north linac (linear accelerator) starts a number 
of laps (up to 5) of acceleration to reach up to about $6 \mathrm{GeV}$ in the main CEBAF machine that looks like a race track. In between the accelerations in the two linacs (north and south) of the machine, the beams are directed around two $180^{\circ}$ magnetic recirculating arcs each with a radius of $80 \mathrm{~m}$ [58]. For acceleration, each linac employs a series of 20 cryomodules, each of which is a cryogenic unit consisting of a series of 8 niobium resonant superconducting RF cavities, vacuum pipes and pumps for cryogenics, and magnetic dipoles/quadrupoles for beam focussing and steering. The cavities in the modules are cooled below the $9 \mathrm{~K}$ superconductivity point by $2.2 \mathrm{~K}^{2} \mathrm{LHe}$ (liquid-Helium) from the central helium refrigerator and the radiation shields are kept cool with $4.5 \mathrm{~K} \mathrm{LHe}$ from an end-station refrigerator. A 5-kW klystron synchronized (to less than $1^{\circ}$ in phase difference) to the master driving RF (at the injector) supplies the RF power to each cavity which creates an oscillating phase gradient along each cavity with the field maxima and minima having the same separation as the spacing between the cavity nodes (see Fig. 12). Since the bunch frequency is in resonance with the RF field, the electrons get a net acceleration. The cavities operate in continuous wave (CW) mode with a gradient of at least $5 \mathrm{MeV}$ per meter so each of the linacs provides a boost of about $600 \mathrm{MeV}$ to the beam. The electron bunches are delivered to the three halls in sequence, and since the bunches can be accelerated to different energies by recirculating ${ }^{3}$ them through the CEBAF different number of times, the three halls can either get the same energy or different multiples of the one-pass energy (about $1.2 \mathrm{GeV}$ ). By controlling the intensities of the three independent lasers shining the photocathode in the injector, electron densities in the corresponding bunches can also be made different to provide different beam currents to the halls $[62,61]$.

To minimize the accelerator hardware resources (tunnel space, cryomodules, magnets etc.) the idea of using the recirculation arcs was implemented in the CEBAF design. The arcs allow for the multiple laps/passes (up to 5) of beams through the linacs for higher energies. Although the bunch lengths are the same for the different passes (enabling the use of the same SRF cavities), their energies being different, they require different bending strengths and, therefore, different bending magnetic fields for each pass. For that reason, there are 5 arcs at the eastern end of the linacs and 4 at the other. A chicane magnet at each end of the linacs separates the multi-energy beam into single energy beams and sends

\footnotetext{
${ }^{2}$ The lower temperature minimizes the BCS energy losses.

${ }^{3}$ Since electrons are extremely light particles, they travel essentially with the same speed as light at energies above $45 \mathrm{MeV}$, thus making it possible to use the same resonant cavities and driving RF frequencies to boost the beam energy in every pass through the CEBAF machine.
} 
them through the different arcs each consisting of a chain of six "periods", with each "period" made of 8 dipoles, 8 quadrupole and 4 sextuple magnets. This optical configuration keeps the beam from degrading due to dispersion and blurring, and also provides a path length that is an integer multiple of the accelerating RF wavelength in addition to helping minimize the energy spread due to the synchrotron radiation [61]. After the beam passes through the south linac, a beam switchyard separator either lets the beam to go through another pass of acceleration, or extracts it with a chicane from the appropriate recirculation arc (after 2 to 5 passes depending on the beam energy requested). After the extraction, a $\frac{1}{3}$ harmonic RF separator, with the help of an oscillating deflecting field, separates the bunches meant for the three different research halls and steers them towards 3 different openings in a Lambert septum. After the separation, the three beams are sent to the appropriate halls - via a straight beam-line to the CLAS detector in Hall B, and via two arcs ${ }^{4}$ (with steering magnets) to the Halls $\mathrm{A}$ and $\mathrm{C}$.

One final point to be noted is that during the beam recirculation process, the bending magnetic fields cause the electrons to undergo spin precessions, the amount of which is a function of the total number of times the beam is recirculated, the energy boost from each linac, and the injector energy. As a result, the maximum (pure longitudinal) polarization is achieved when the precession angle $\theta$ is a multiple of $\pi$. This condition can happen simultaneously in the three halls if particular combinations of beam energies are chosen. However, that is not always a feasible choice and, therefore, in many cases, a fraction of transverse polarization can be present. But, this does not affect the experimental results much because the contributions from the transverse polarizations are suppressed by a factor of $1 / \gamma$ [62]. In addition, a Wien filter is used in the injector which allows further control of the spin direction $[63,64]$.

\footnotetext{
${ }^{4}$ The bendings here are exploited for the precise measurements of the beam energies
} 


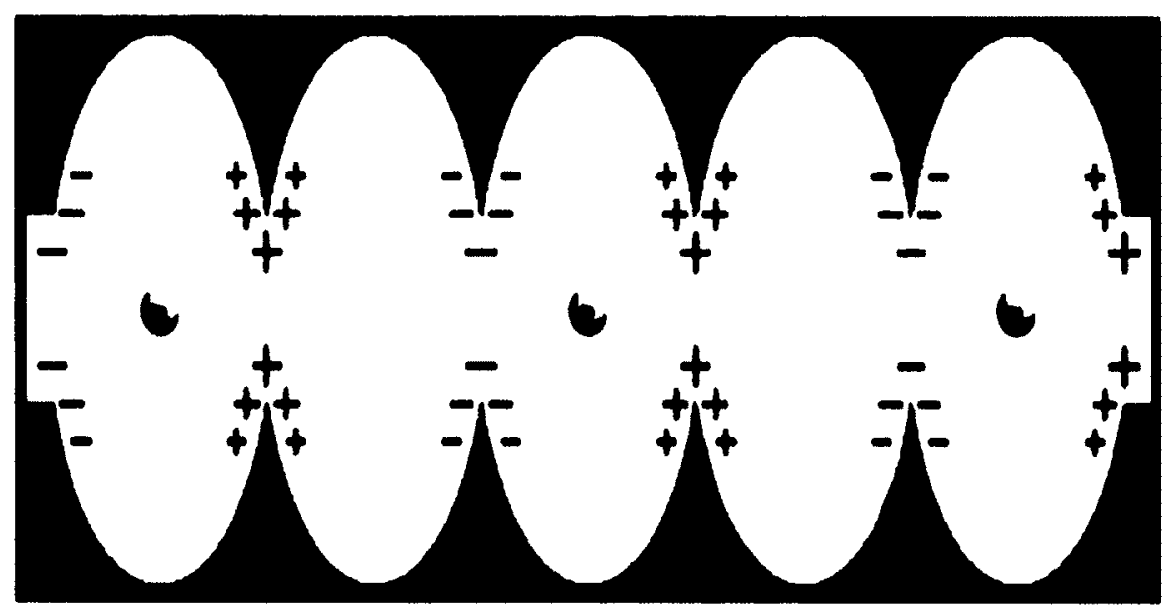

FIG. 12. The acceleration is provided by establishing standing waves tuned such that an electron always experienced a positive electric force while passing through the cavity

\subsection{HALL-B BEAMLINE DEVICES}

After the beam extracted from the CEBAF machine is directed towards the Hall $\mathrm{B}$, it passes through a number of devices before reaching and after exiting ${ }^{5}$ the EG4 target and the CLAS detector. As depicted in the schematic Fig. 13, the devices used before the beam reaches the targets are a Moeller Polarimeter, 3 Beam Position Monitors (BPMs), and 3 Harp Scanners, and the one that comes after the CLAS is a Faraday Cup.

The beam polarization is measured at the injector using a Mott polarimeter, but we cannot rely on that because the polarization may differ due to the spin precession mentioned in the previous section. Therefore, a Moeller polarimeter installed at the entrance of Hall $B$ is used to make beam polarization measurements.

Moeller polarimetry is based on Moeller scattering of beam electrons from the atomic electrons in an iron (or iron-alloy) target polarized by an external magnetic field. The method is an invasive one, and therefore requires separate Moeller data runs ( $\sim 30$ minutes long) taken periodically throughout the experiment.

The polarimeter (see Fig. 14) consists of a target chamber with a $25-\mu \mathrm{m}$ thick permendur ${ }^{6}$ foil oriented at $\pm 20^{\circ}$ with respect to the beam line and longitudinally polarized

\footnotetext{
${ }^{5}$ Applies only to the electrons that didn't get scattered in the target

${ }^{6}$ Permundur is an alloy of $49 \% \mathrm{Fe}, 49 \% \mathrm{Co}$, and $2 \% \mathrm{Va}$.
} 


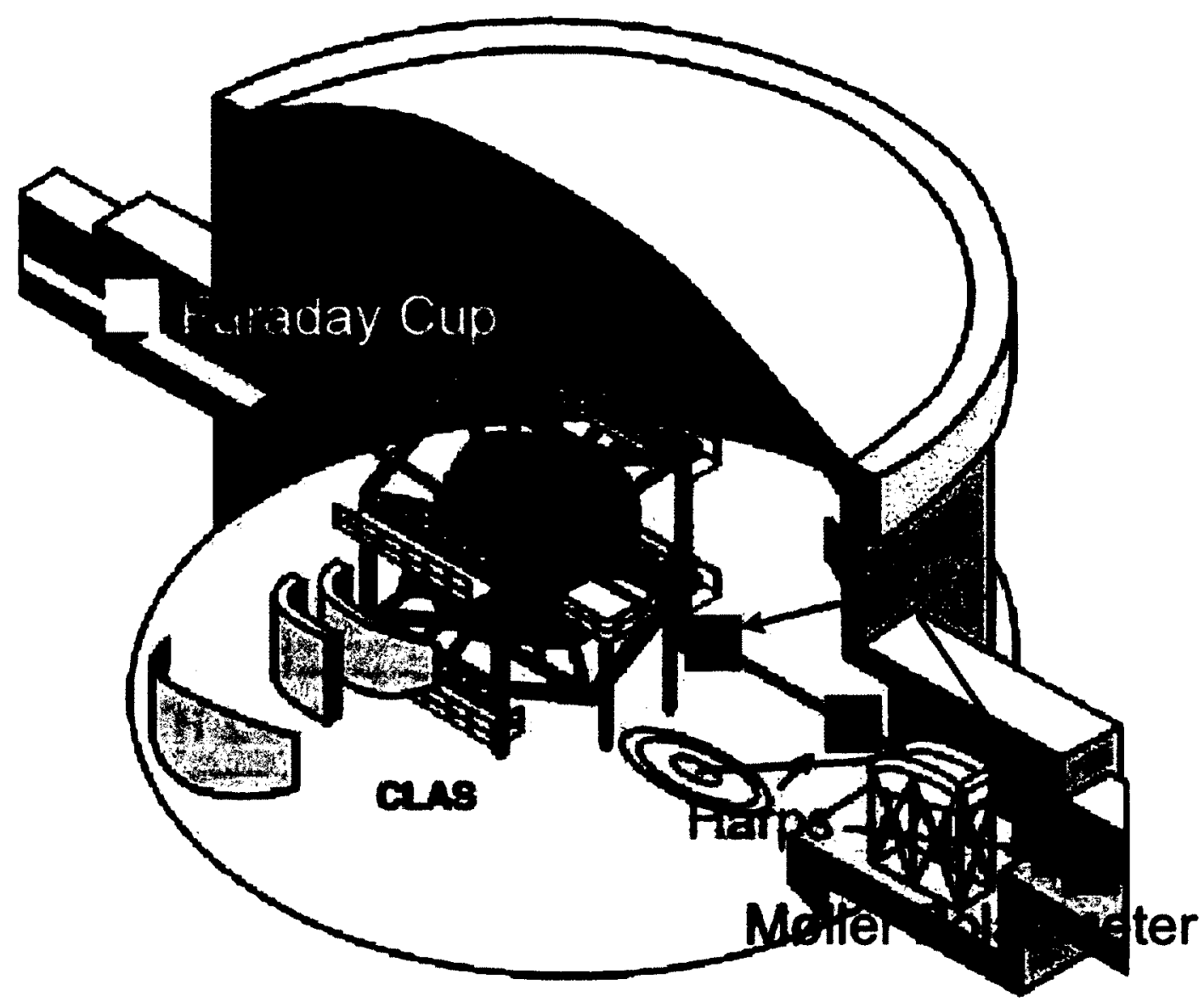

FIG. 13. CLAS in the Hall-B beamline

to $7.5 \%$ by a $120 \mathrm{G}$ Helmoltz magnet. Two quadrupoles separate the scattered electrons according to their polarizations. The electrons then enter one of two lead/scintillator/photomultiplier tube combinations for detection. Elastic electron-electron scattering coincidences are used to determine the polarization. The differential scattering cross-section, in terms of the permendur target polarization $\left(P_{t}\right)$ and beam polarization $\left(P_{b}\right)$, is given by

$$
\frac{d \sigma}{d \Omega} \propto\left(1+\sum_{i, j=x, y, z} P_{i}^{t} A_{i j} P_{j}^{b}\right),
$$

where,

$$
\begin{aligned}
& A_{y y}=-A_{x x}=\frac{\sin ^{4} \theta_{C M}}{\left(3+\cos ^{2} \theta_{C M}\right)^{2}}, \\
& -A_{z z}=\frac{\left(7+\cos ^{2} \theta_{C M}\right) \sin ^{2} \theta_{C M}}{\left(3+\cos ^{2} \theta_{C M}\right)^{2}}
\end{aligned}
$$




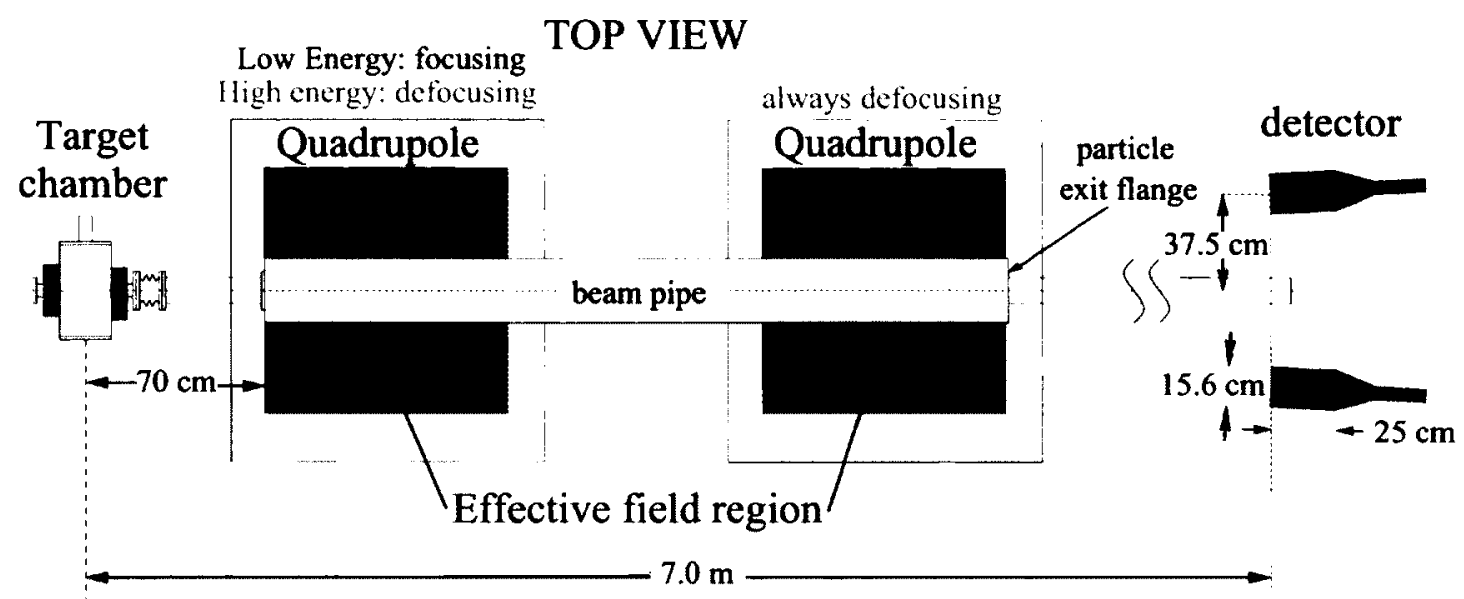

FIG. 14. A schematic diagram of the Hall B Moeller Polarimeter (from the top view perspective).

$$
A_{i j, i \neq j} \approx 0
$$

Here, $\theta_{C M}$ is the scattering angle in the center-of-mass $(\mathrm{CM})$ frame, $\mathrm{z}$ is defined as the beam axis, and the ee scattering plane is defined to be the $\mathrm{xz}$ plane. Using knowledge of the scattering kinematics and $P_{t}$ (from the detectors and foil alignment, respectively), the beam polarization, $P_{z}^{b}$, can be determined. The Moeller measurement typically had an absolute statistical uncertainty of $1 \%$ and a systematic uncertainty of $\sim 2 \%$. In practice, normalization to the elastic scattering asymmetry is used to determine the beam times target polarizations; the only actual use for the Moller measurements in this analysis is for consistency checks on the $P_{b} P_{t}$ measurements [61].

During the experiment, it is very important to have systems to keep track of the stability of beam alignment and the current level. There are three such systems which are known as Beam Position Monitors (BPM) and are located at 36.0, 24.6 and 8.2 m upstream from the CLAS center. Each of them monitors three things - the $X$ and $Y$ position of the beam and the beam current - with position and current resolutions of 10 microns and $50 \mathrm{pA}$ respectively. Each BPM has $3 \mathrm{RF}$ cavities operating at $1497 \mathrm{MHz}$ to monitor the three variables. The monitoring data is taken at a rate of $1 \mathrm{~Hz}$ and are used in a feedback loop to keep the beam centered on target $[65,66,67,61]$.

The next set of the beam-monitoring devices are the three Harp Beam Profile Monitors, which are located at 36.7, 22.1 and 15.5 m upstream of the CLAS center. Each of them measures the profile and diameter of the electron beam through periodic harp scans. A scan 
is done by slowly moving a cross-hair of two thin wires $(20 \mu \mathrm{m} \mathrm{W}, 50 \mu \mathrm{m} \mathrm{W}$ and $100 \mu \mathrm{m}$ $\mathrm{Fe}$, respectively) through the beam. A beam profile is then reconstructed by detecting the electrons scattered off these wires using photomultipliers (PMTs) located at $10 \mathrm{~cm}$ distance from the beam line. Past diameter measurements have shown an RMS of around $80 \mu \mathrm{m}$ indicating that most of the beam falls within a $200 \mu \mathrm{m}$ diameter. Like Moeller measurements, harp scans are invasive, and therefore, not done during data collection [61].

Finally, the total integrated beam charge, which is a crucial part of data required for the calculation of experimental cross-sections, is measured by a Faraday Cup (FC), which is located at the end of the beam line ( $29.0 \mathrm{~m}$ downstream from the CLAS center) as a part of the beam dump ${ }^{7}$. The device is a horizontal, 75 radiation lengths long $(4000 \mathrm{~kg})$ lead $(\mathrm{Pb})$ cylinder with a diameter of $15 \mathrm{~cm}$ which is connected to an isolated electrical circuit to measure the collected charge, which, in turn, is connected through a logic gate to the CLAS data acquisition system (DAQ) to record two types of measurements - one for the total (un-gated) charge and the other for the "detector-live-time" (gated) charge which ignores the electrons that arrive while the readout system (DAQ) is busy. Separate recordings are made for each beam helicity bucket by gating the device with the main $R F$ frequency.

\subsection{EG4 TARGETS}

This experiment took two sets of data - one each for polarized hydrogenated and deuterated ammonia (i.e., $\mathrm{NH}_{3}$, and $\mathrm{ND}_{3}$ ) targets. The choice of the target material was a compromise between the desire for a pure proton/deuteron target, and the practical necessities of materials that provide better polarization and resistance to radiation damage [68]. For each of the beam energy settings, for the purpose of background studies and systematic checks, some data were also collected with the following three types of unpolarized targets - carbon-12, target cup with liquid-helium-4 only, and empty target cup without helium.

Even though the target sample itself was tiny in size $(1.0 \mathrm{~cm})$, the fact that it needed to be polarized made the whole target system big with a host of accessories. The system consisted of a superconducting magnet, a one-Kelvin refrigerator, a target insert/stick (carrying the target samples), a microwave system and an NMR system, with the entire assembly, including the pumping system, attached to a rail-mounted cart which could be

\footnotetext{
${ }^{7}$ Because only a tiny fraction of the beam is lost due to the scattering at the target as well as on other beamline materials, the FCup measurement is not much different from the actual incident charge (not exactly true for low beam energies).
} 


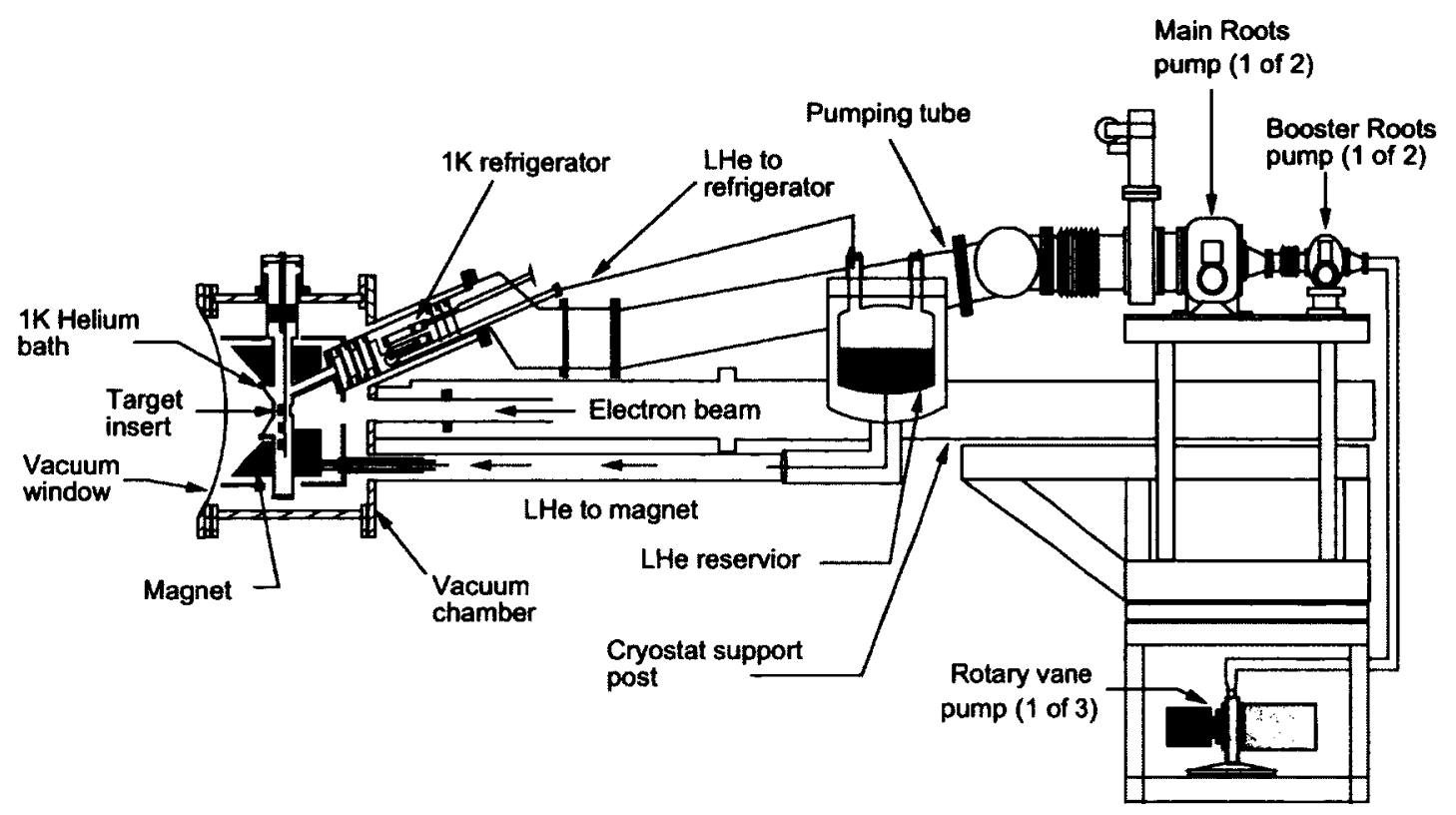

FIG. 15. Sectional view (from the beam-left side) of the polarized target system

rolled into or out of the CLAS within a matter of minutes [69].

One may be inclined to think that we could achieve the intended nuclear polarization simply by forcing the alignment of proton spins in our target sample by placing it in a very high magnetic field (B) and at a very low temperature (T). As per Boltzmann statistics such a thermal equilibrium (TE) polarization (we could call it the Static Nuclear Polarization) would be given by

$$
P_{T E}=\frac{e^{\frac{\mu B}{k T}}-e^{\frac{-\mu B}{k T}}}{e^{\frac{\mu B}{k T}}+e^{\frac{-\mu B}{k T}}}=\tanh \left(\frac{\mu B}{k T}\right)
$$

At a field of $5 \mathrm{~T}$ and temperature of $1 \mathrm{~K}$, the proton polarization would be only $0.3 \%$ only (free electron gas polarization would be near $100 \%$ due to the 660 times higher magnetic moment), which obviously is not practical for experiments [68]. For this reason, the technique of microwave driven Dynamic Nuclear Polarization (DNP) [70] was used to enhance the polarization. At the $5 \mathrm{~T}$ field and $1 \mathrm{~K}$ temperature, DNP can produce polarizations as high as $96 \%$ and $46 \%$ in $\mathrm{NH}_{3}$ and $\mathrm{ND}_{3}$ targets respectively [69].

DNP is one of several techniques for hyper-polarization (polarization of nuclear spin beyond thermal equilibrium) of a given material. DNP results from the spontaneous transfer of spin polarization from electrons to nuclei which takes place when the electron spin 


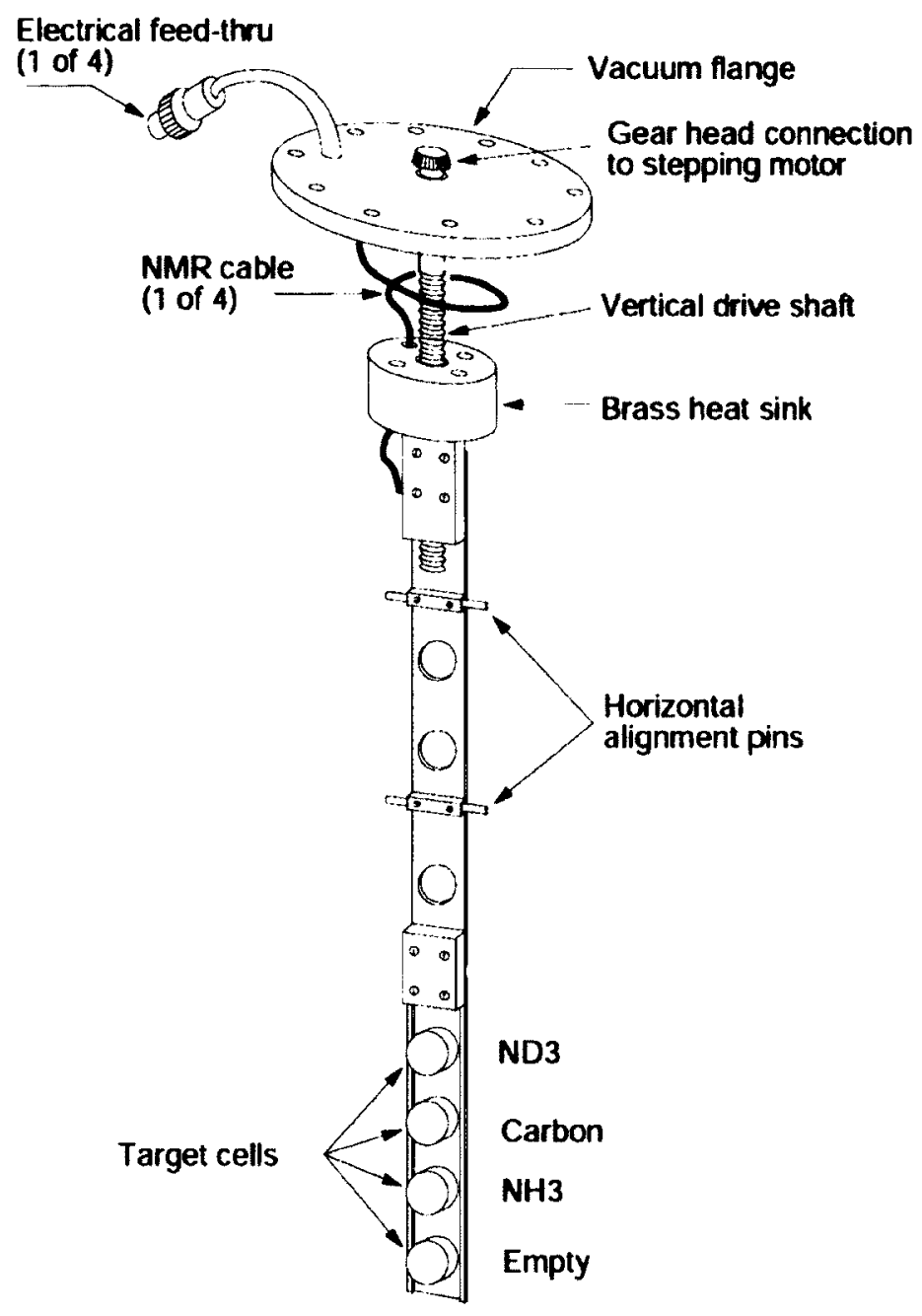

FIG. 16. Schematic of Hall-B polarized target insert

polarization deviates from its thermal equilibrium value [70]. In our case, such a deviation is induced by continuous microwave irradiation close to the corresponding electron paramagnetic spin resonance (EPR or ESR) frequency.

The basic idea involves using the hyper-fine splitting as shown in Fig. 17 which results from the spin-spin coupling of free electrons to the nuclei (protons or deuterons) we wish to polarize. By irradiating the target with microwaves of frequencies that match the energy gaps seen in the diagram, transitions can be induced to flip the spin of the proton/deuteron along with the spin of the electron. As shown, the " $\Downarrow "$ aligned electron-nucleus state can be flipped to the " $\Uparrow$ " aligned state using microwaves. Also, by using a different 


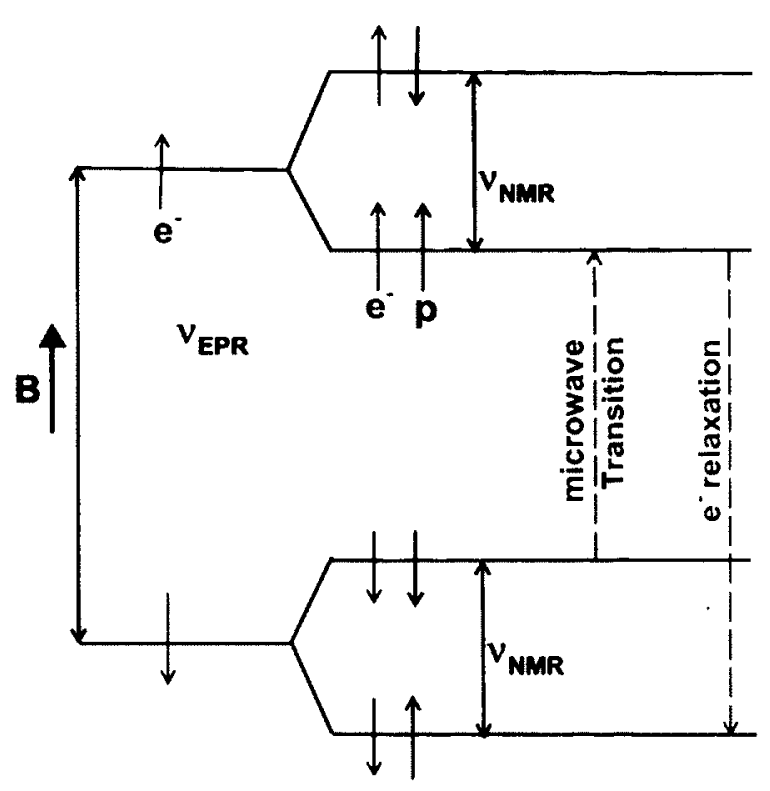

FIG. 17. Electron energy levels arising from the hyperfine splitting due to the coupling of free electron and proton spins.

microwave frequency matching the corresponding energy gap, it is possible to flip the

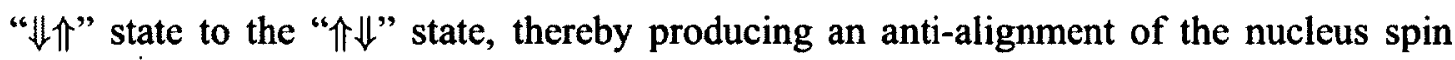
without changing the magnetic field. Thus both positive and negative polarizations can be produced using the same field. After these upward transitions, the electron relaxes back to the lower energy spin state. Due to the stronger coupling of electrons with the lattice than that of nuclear spins, the electron spins flip back much more quickly (the relaxation times at $1 \mathrm{~K}$ are $\approx 10^{-3} s$, and $\approx 10^{3} s$ for electrons and protons respectively) [68] and the same electron now can be used to polarize another nucleus and so on. This way, the nuclei near the free electrons accumulate into one spin state producing a net polarization which propagates throughout the target volume via the process of spin diffusion.

In order to provide free electrons for the spin-spin coupling required by the DNP, our targets were doped with paramagnetic centers (radicals) twice. First, in what is called a warm dose, the target at $80 \mathrm{~K}$ is irradiated with an electron beam in a smaller accelerator, which produces radicals such as $\mathrm{NH}_{2}$ from $\mathrm{NH}_{3}$. Finally, the cold dose (at $1 \mathrm{~K}$ ) of the CEBAF beam produces different radicals such as atomic $\mathrm{H}$.

The $5 \mathrm{~T}$ field required by the DNP is produced by a superconducting Helmholtz magnet which is kept at $4.2 \mathrm{~K}$. The magnet produces a field uniform to better than $1 \times 10^{-4}$ (enough 
to resolve the ESR linewidth of the paramagnetic radicals) over a cylindrical volume that is $20 \mathrm{~mm}$ long, $20 \mathrm{~mm}$ in diameter and is centered at the target location.

The polarized target material is kept at a temperature of about $1 \mathrm{~K}$ by immersing it in bath of liquid ${ }^{4} \mathrm{He}$. The low temperature is achieved with a cooling system that consists of a system of Roots and rotary-vane vacuum pumps, a $1 \mathrm{~K}$ refrigerator and an evaporation chamber. An insert as shown in Fig. 16 is used to hold all the target materials in four cylindrical cups roughly $1 \mathrm{~cm}$ in diameter and length. The insert is introduced into the evaporation chamber and then remotely controlled by a stepping motor to move each of the four targets onto the designated target position along the beam path. A gold-plated rectangular horn connected to a mircowave generator via waveguides, which is fixed rigidly inside the evaporation chamber and facing towards the designated target position, continuously delivers the needed microwave power, thus driving the DNP to produce the needed polarization.

A continuous wave NMR (Nuclear Magnetic Resonance) system is used for online (real time) monitoring of the target polarizations. The NMR system is essentially an RCL circuit with its coil wrapped around the cylindrical cell containing the polarized target material [61].

Two software systems installed on separate computers were used to control and monitor the operation and performance of the target system. Labview 5.2 was one of the two which operated from a PC located in the experimental hall and was primarily dedicated to NMR monitoring. The second program running on a VME-based single board computer in the hall was known Experimental Physics and Industrial Control Software (EPICS), which was used to control the cryogenic subsystems. The system handled most processes automatically, but it could also be monitored and controlled from outside the hall by accessing its graphical user interface from any Unix/Linux workstation on site via the Jlab Local Area Network [68].

\subsection{CEBAF LARGE ACCEPTANCE SPECTROMETER (CLAS)}

The CEBAF Large Acceptance Spectrometer (CLAS) (see Figs. 18 and 19), which is housed in Hall B of Jefferson Lab, is a nearly $4 \pi$ detector, which makes it ideal for studying multi-particle final-state reactions induced by photons and electrons at luminosities up to $10^{34} \mathrm{~cm}^{-2} \mathrm{sec}^{-1}$ [71]. The detector is divided into six identical sectors (each functioning as an independent magnetic spectrometer) with a superconducting coil located in between each two of them (see next section). Each sector has three layers of drift chambers (DC) 


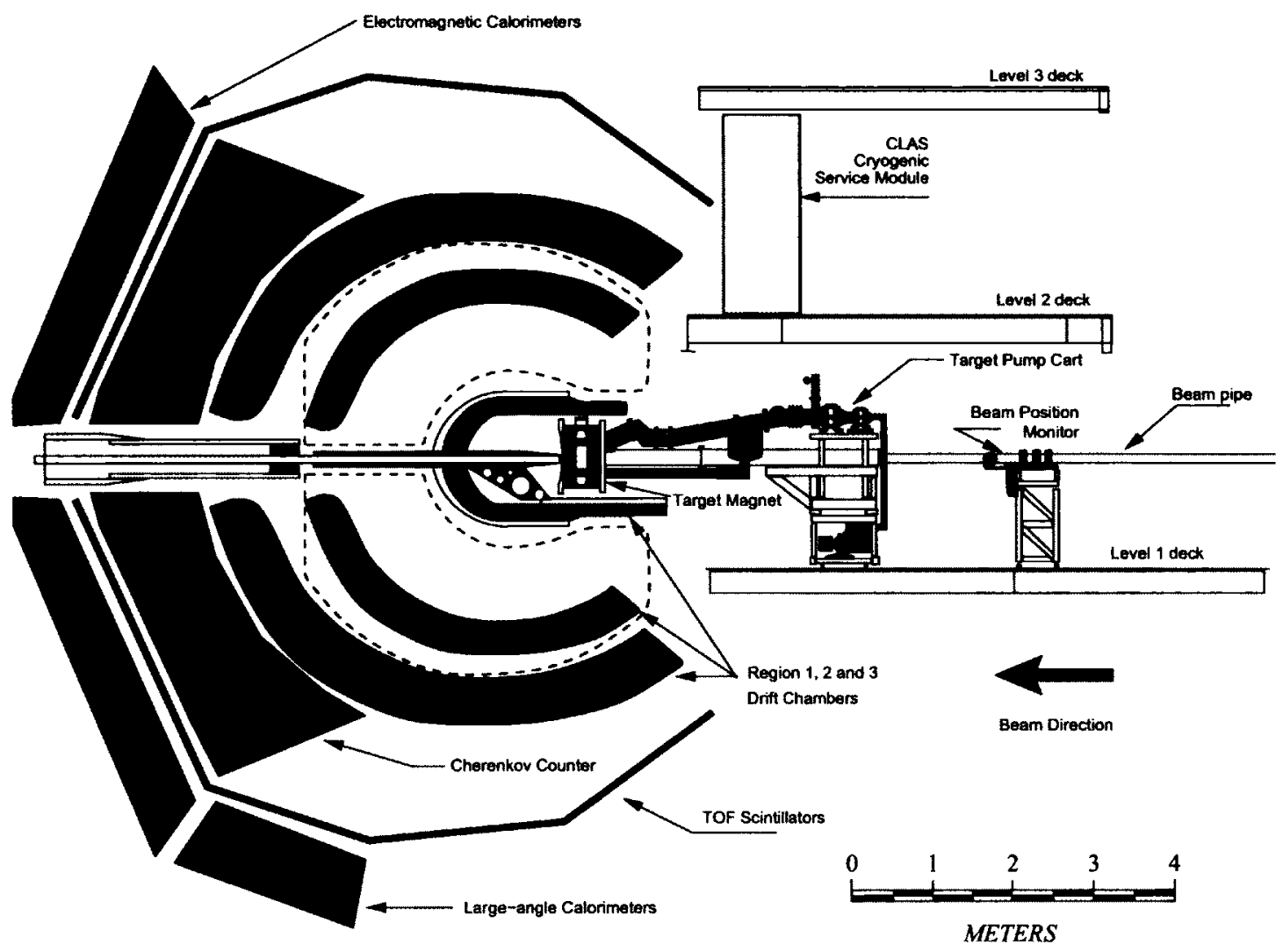

FIG. 18. EG4 Experimental Setup showing a cross-sectional view of the CLAS detector along with the polarized target system in place.

and one layer of time of flight (TOF) or scintillation counters (SC), which cover the full detector acceptance. Each sector also has a Cherenkov counter $(\mathrm{CC})$ and an electromagnetic calorimeter (EC) installed in the forward region of $8^{\circ}$ to $45^{\circ}$.

A new (Moeller) Shield made of Tungsten (higher density than Lead) was put in place to suppress low-momenta background electrons (also called Moeller electrons because they originate due to the Moeller scattering from the atomic electrons), optimized for small angle $(\theta)$ operation at high luminosity.

\subsubsection{TORUS MAGNET}

The six superconducting coils placed one each in the gaps between the six independently instrumented CLAS sectors form a toroidal configuration. This arrangement allows for a central magnetic field-free region which can be very useful for purposes such as the 


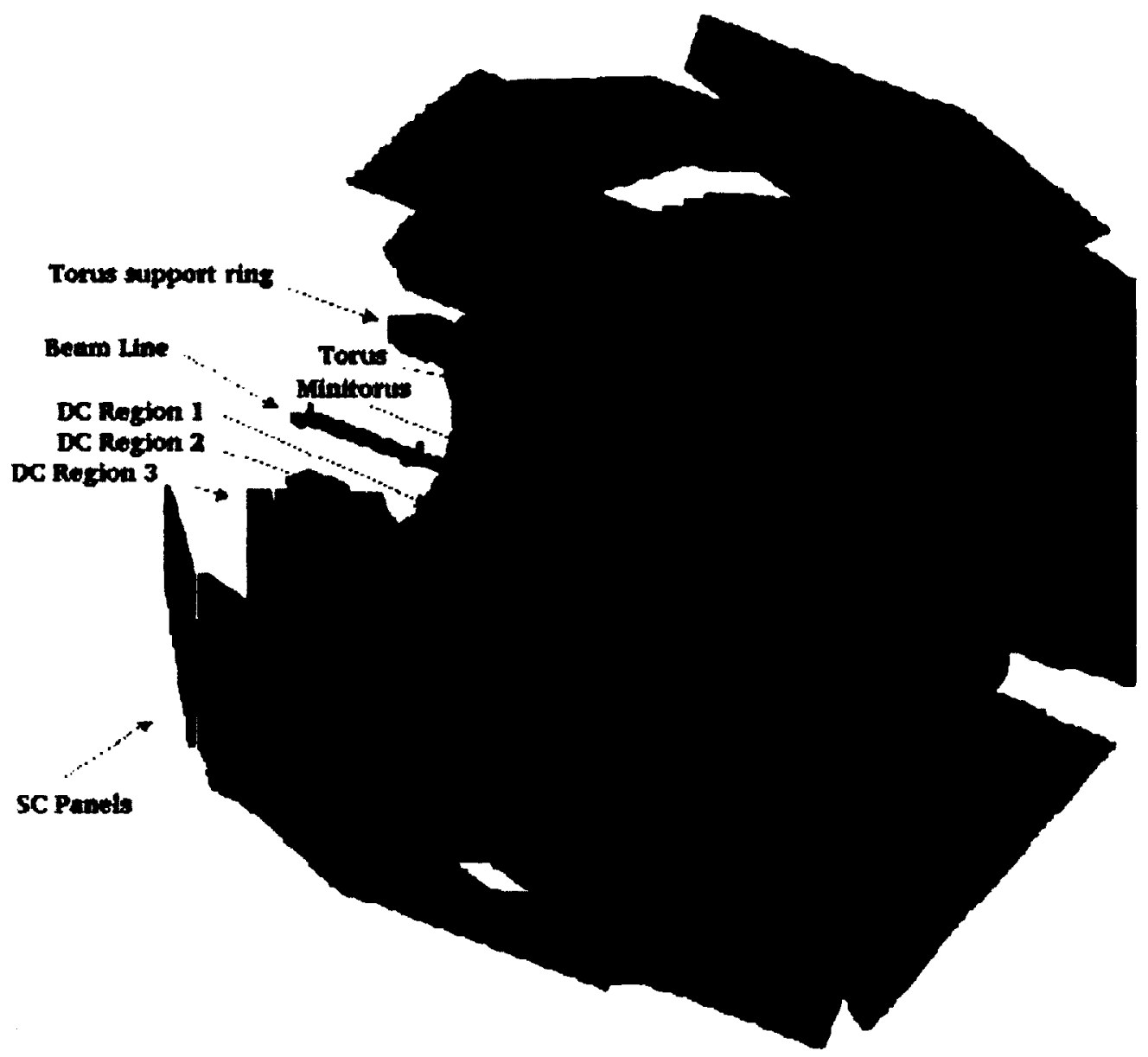

FIG. 19. Cut-away view of CLAS detector

insertion of a polarized target [69].

The torus magnet setup produces a magnetic field up to 2.7 Tesla in the $\phi$-direction, surrounding the beam line. The magnetic field causes charged particles to bend when they are moving through the detector. If the electron bends towards (away from) the beam line, we call it the in-bending (out-bending) setting. This allows one to determine the charge type and measure the momenta of charged particles according to the bending in their trajectories.

In order to perform an absolute cross-section measurement, the CLAS-setup with a few modifications was used. In contrast to the usual in-bending torus configuration, an out-bending torus field was applied in this experiment in order to make measurements 


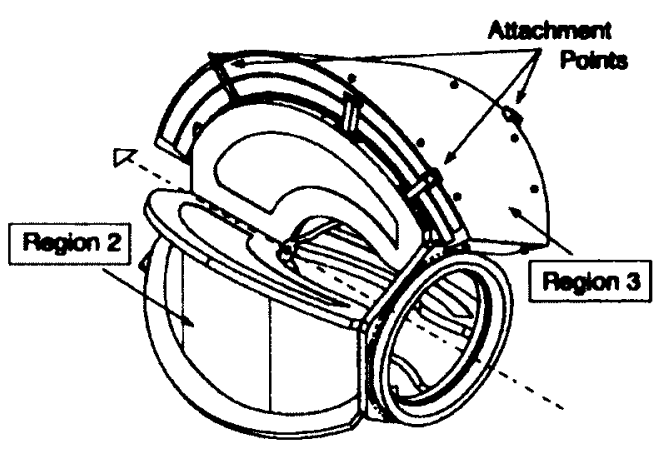

(a) Torus

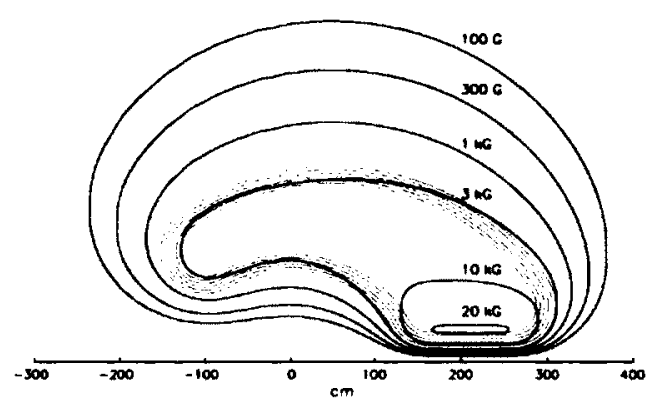

(b) Torus Magnetic Field Contour

FIG. 20. The CLAS Torus superconducting magnet (left) and the contour of its field.

down to as low as six degrees so as to achieve as low- $Q^{2}$ measurements as possible.

\subsubsection{DRIFT CHAMBERS (DC)}

Charged particles in CLAS are tracked by a set of drift chambers (DC). A drift chamber has thin wires fixed in a volume filled with a special gas in such a way that there is a posititve central (sense) wire surrounded by six negative (field) wires to form hexagonal cells. Inside these cells a traversing charged particle ionizes the gas and the ionization electrons drift to the sense wires. The connected electronics measures the charge of the signals and the corresponding times the signals appear. The difference between this signal arrival time and the time when the particle traversed the cell (measured by other detectors) is used to reconstruct the particle impact points in the chamber virtual planes [72]. Using such impact points, one can re-construct the track of the traversing particle.

The CLAS drift chambers are arranged in three regions: Region 1 is located closest to the target, within the (nearly) field free region inside the Torus bore, and is used to determine the initial direction of charged particle tracks. Region 2 is located between the six super-conducting Torus coils, in the region of strong toroidal magnetic field (up to 2.7 Tesla [73]), and is used to obtain a second measurement of the particle track at a point where the curvature is maximal, to achieve good energy resolution. Region 3 is located outside the coils, again in a region with low magnetic field, and measures the final direction of charged particles headed towards the outer TOF, CC and the EC counters. All three regions consist of six separate sectors, one for each of the six sectors of the CLAS. So, there are 18 different drift chambers in CLAS [74]. 
The DC information is important for energy, momentum and angle determination as well as for particle identification. In this experiment, the drift chamber system was used in the standard CLAS configuration.

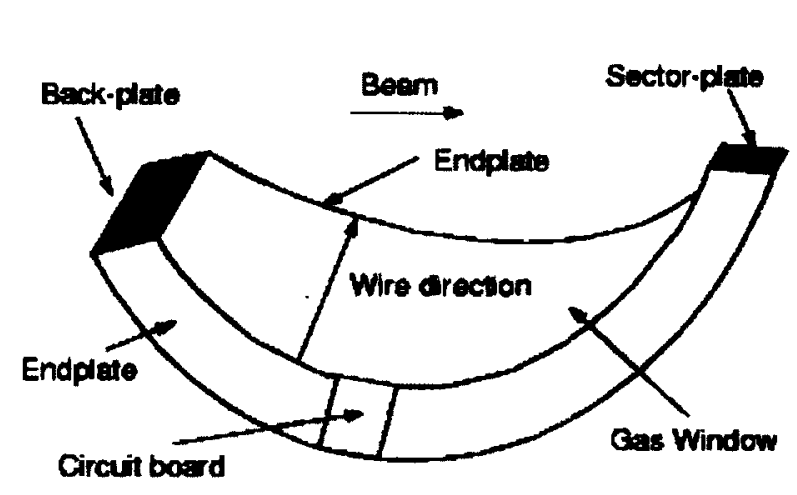

(a) A DC sector

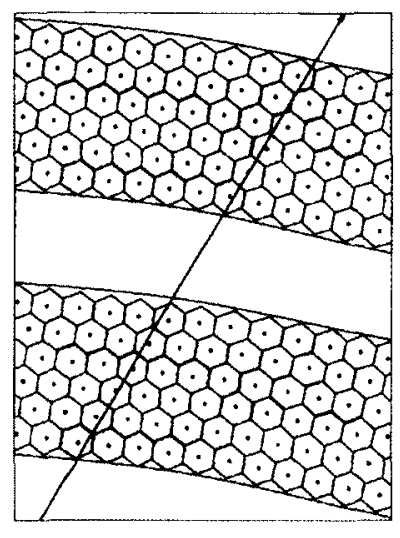

(b) DC cells

FIG. 21. Different parts of a DC sector (left) and a section of such a sector showing arrays of DC cells as well as those that fired when a charged particle passed through them.

\subsubsection{SCINTILLATION COUNTERS (SC) OR TIME OF FLIGHT (TOF) SYSTEM}

The TOF (SC) system (here used in the standard CLAS configuration) provides a highresolution ( $\sim 140 \mathrm{ps}$ ) timing measurement that can be used for velocity and mass calculation purposes. A scintillation counter measures ionizing radiation with a transparent crystal, usually phosphor or plastic (CLAS uses $5 \mathrm{~cm}$ thick BC408 [73]) that fluoresces when struck by the ionizing radiation. A sensitive photo-multiplier tube (PMT) detects the light from the crystal. Scintillation counters typically have a poor spatial resolution but a very good time resolution. They are also continuously sensitive, and are therefore often used as triggers for other types of detectors.

In EG4, the CLAS was triggered by requiring a coincidence between the forward electromagnetic calorimeter (EC) and the new INFN Cerenkov counter (CC) which was installed only in the sixth sector [34]. 


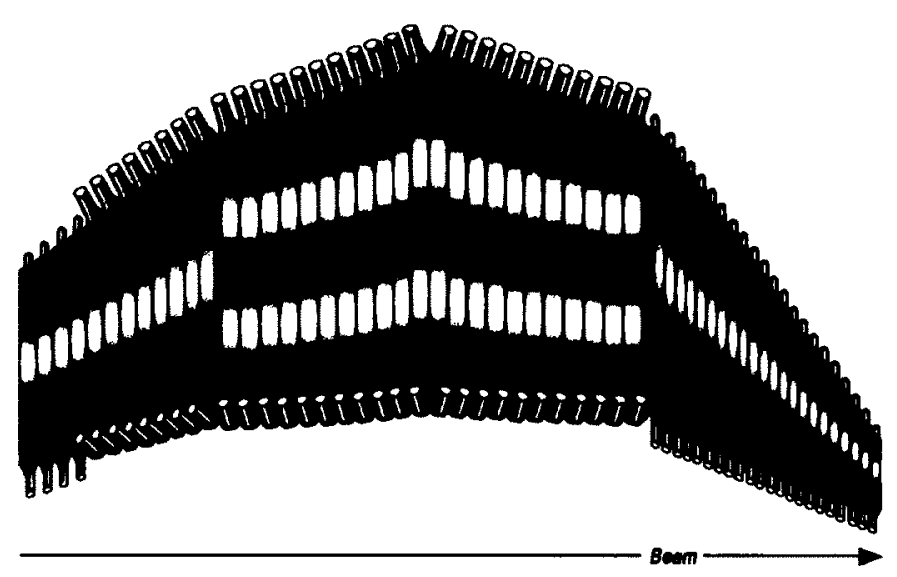

FIG. 22. TOF system

\subsubsection{CHERENKOV COUNTERS (CC)}

The Cherenkov Counter (CC) serves the dual function of triggering on electrons and separating electrons from pions (or identifying charged particles). These detectors use the light emitted by Cherenkov radiation (emission of light when the charged particle travels faster than light in that medium) to measure the particle velocity (and, therefore, $\beta=v / c$ ). The knowledge of $\beta$ combined with the particle momentum (from the tracking detectors) determines the particle's mass, thus giving us information on the particle identification. The index of refraction (n) is carefully optimized for the particle masses and momentum range of the experiments in question. Threshold counters record all light produced, thus providing a signal whenever $\beta$ is above the threshold $\beta_{t}=1 / n$. In the standard configuration, CLAS uses one Cherenkov threshold detector in each of the six sectors in the forward region from $8^{\circ}$ to $45^{\circ}$.

\section{New $\mathrm{CC}$ in the $6^{\text {th }}$ Sector}

The standard CLAS Cherenkov detectors (as shown by Figs. 24 and 23) were designed such that their optics, geometry, module position and mirror orientation were optimized for low rate high $Q^{2}$ experiments that mostly use(d) electron in-bending torus fields. The design was a compromise between the desired kinematic coverage and the complexities of the CLAS detector system including the effect of the torus field. As a consequence, light collection is constrained causing the number of photoelectrons to be strongly dependent on scattering angles, and making the detection efficiency non-uniform, and strongly reduced 


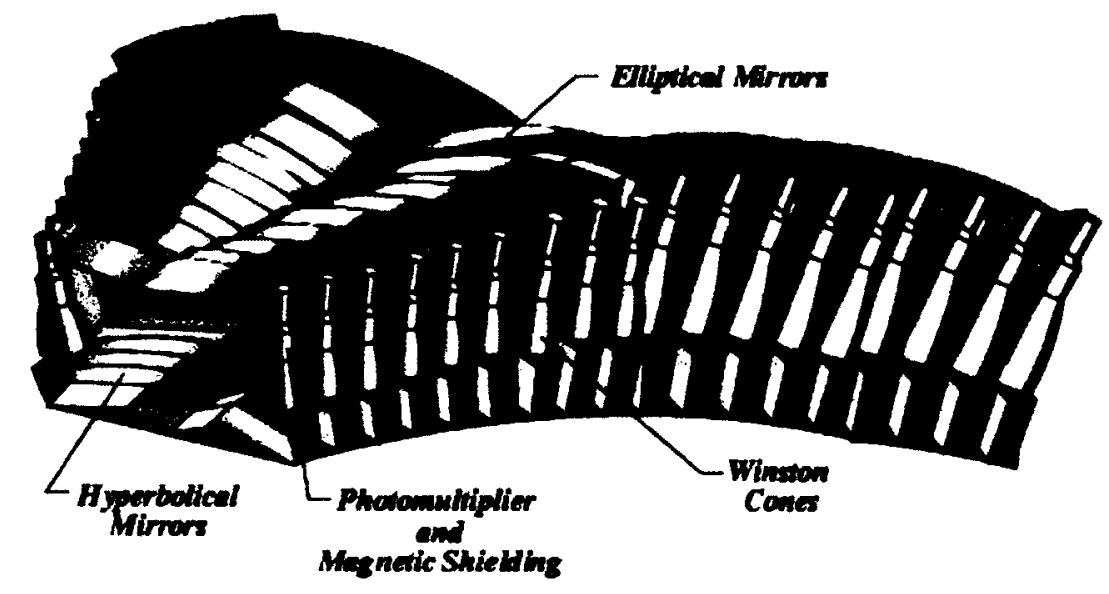

FIG. 23. The computer rendered image of the Standard CLAS Cherenkov Counter

in some regions (for example, up to $30 \%$ drop in the middle of the sector and at forward angles) [34]. While it would still be possible to detect electrons, the use of the existing CC would mean that the absolute cross-section measurement would require large and complex corrections which are difficult to evaluate. That would significantly contribute to the systematic uncertainties, thus not meeting the proposed high accuracy requirement of the measurements.

In order to avoid having all those $\mathrm{CC}$-related issues in the new measurements, a new gas threshold cherenkov counter (designed and built by INFN - Genova, Italy) was installed in the sixth sector. This new CC detector (see Fig. 25 for its CAD rendition) is specifically optimized for the out-bending field configuration, which is necessary to reach the desired low momentum transfer (measurements down to 6 degrees). The detector uses the same radiator gas $\left(C_{4} F_{10}\right.$ - perfluorobutane) and the same gas flow control system as the old one, but it uses a different design. In the new $\mathrm{CC}$, the number of $\mathrm{CC}$-modules is now 11 instead of the 18 in the standard ones. In order to maximize the light collection, a single reflection design (see Fig. 3.26(b)) using spherical mirrors is used (the standard $\mathrm{CC}$ used double relections from elliptical and hyperbolic mirrors). The geometry, the size, the mirror size, position, and orientation, the dimensions as well as the assembly of the modules were optimized for the experiment and the performance study was done using a complete GEANT simulation [34].

This new detector achieves a very high and uniform electron detection efficiency $(\approx$ $99.9 \%$ ) in most of its central (fiducial) region, to allow for the measurement of the absolute 


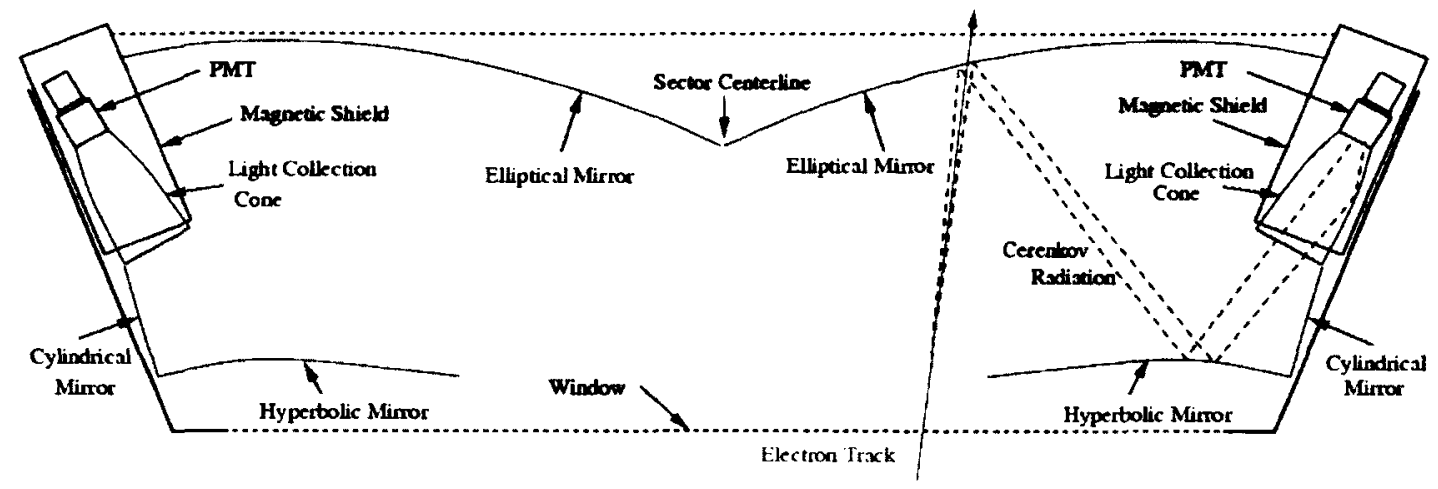

FIG. 24. The schematic diagram of a CLAS Cherenkov Counter (CC) module showing mirrors, PMTs and the light reflections.

cross-section with minimal corrections and a high pion rejection ratio (of the order of $10^{-3}$ ). Due to the high electron rate at low $Q^{2}$, the $\phi$ coverage can be lowered, while still having a large counting rate. Therefore, for reasons of limited data storage capability, and also for the fact that only the sixth sector had the required new $\mathrm{CC}$, only the sixth sector events were collected, stored and subsequently used for data analysis [57].

\subsubsection{ELECTROMAGNETIC CALORIMETERS (EC)}

Each CLAS sector has an electromagnetic sampling calorimeter (EC) in the forward region $\left(8^{\circ}<\theta<45^{\circ}\right)$. These electromagnetic shower calorimeters are optimized for measuring the energies and positions of electrons and gammas [71]. EC helps to discriminate electrons from hadrons and photons from neutrons. When a high-energy electron or photon $(\gamma)$ passes through, a fraction of its energy is deposited in the form of an electromagnetic shower (because of Bremsstrahlung and electron-positron pair production). This shower produces a signal (in the scintillators - the active material) proportional to the energy deposit, which is recorded by the EC read-out. The calorimeter is made of alternating layers of scintillator ( $\mathrm{SC}$ ) strips (36 strips per layer) and lead $(\mathrm{Pb})$ sheets with a total thickness of 16 radiation lengths. In order to match the hexagonal geometry of the CLAS, the $\mathrm{Pb}-\mathrm{SC}$ sandwich has the shape of an equilateral triangle. There are 39 layers in the sandwich, each consisting of a $10 \mathrm{~mm}$ thick scintillator followed by a $2.2 \mathrm{~mm}$ thick lead sheet.

The calorimeter has a "projective geometry" in which the area of each successive layer increases. This minimizes shower leakage at the edges of the active volume and minimizes 


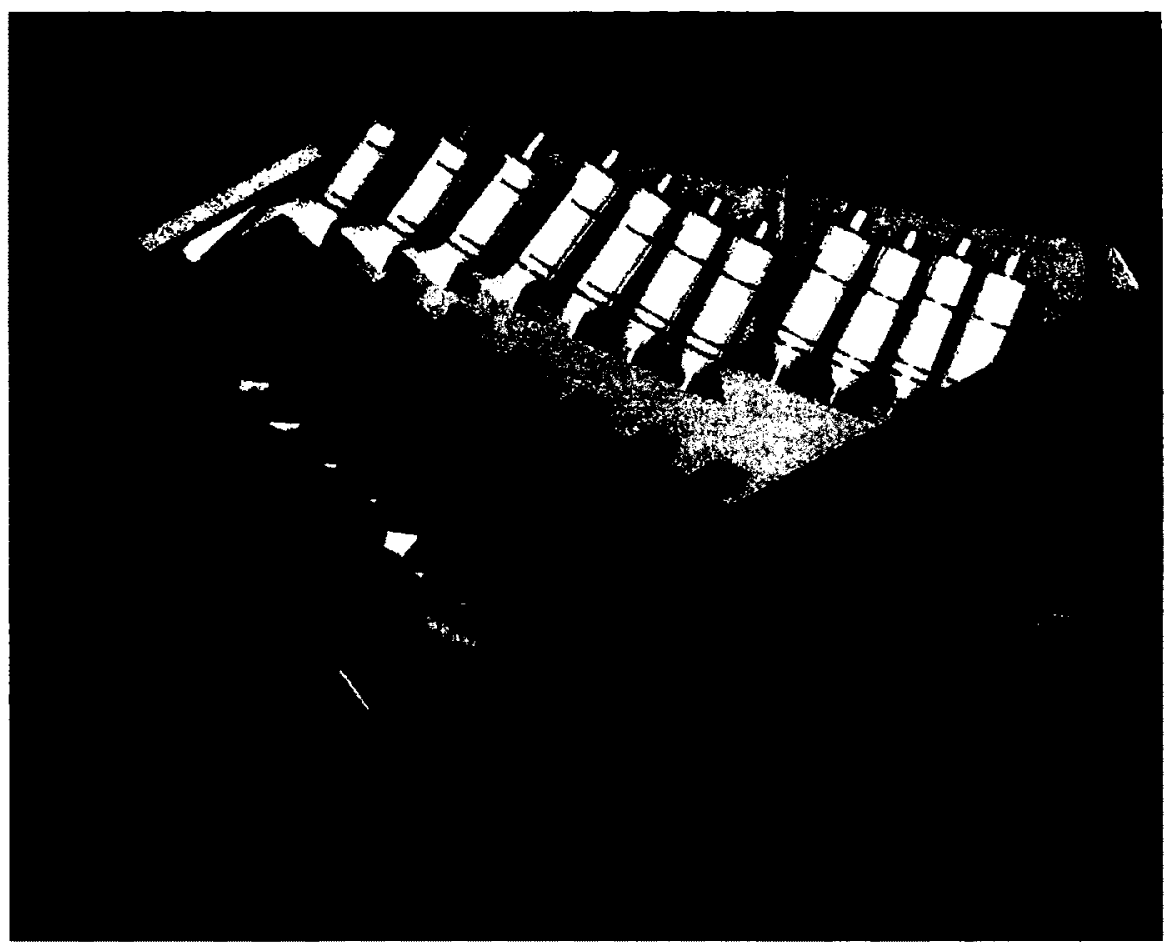

FIG. 25. The new Cherenkov counter (courtesy of INFN, Genova)

the dispersion in arrival times of signals originating in different scintillator layers. The active volume of the sandwich thus forms a truncated triangular pyramid with a projected vertex at the CLAS target point 5 meters away and an area at the base of $8 \mathrm{~m}^{2}$. The projective geometry maximizes position resolution for neutral particles.

For the purposes of readout, each SC layer is made of 36 strips parallel to one side of the triangle, with the orientation of the strips rotated by $120^{\circ}$ in each successive layer. Thus there are three orientations or views (labeled $U, V$, and $W$ ), each containing 13 layers, which provide stereo information on the location of energy deposition. Each view is further subdivided into an inner ( 5 layers) and outer ( 8 layers) stack, to provide longitudinal sampling of the shower for improved hadron identification (or electron-pion discrimination; the electron-pion rejection factor is $\sim 0.01$.). Each module thus requires 36 (strips) $\times$ 3 (views) $\times 2$ (stacks) $=216$ PMTs. Altogether there are 1296 PMTs and 8424 scintillator strips in the six EC modules used in CLAS. The intrinsic energy resolution for showering particles is $10 \% / \mathrm{E}$, with approximately a $3 \mathrm{~cm}$ position resolution at $1 \mathrm{GeV}$. These detectors have up to $60 \%$ efficiency for detecting high momentum neutrons [73].

With its good energy and position resolution, the main functions of EC are: 


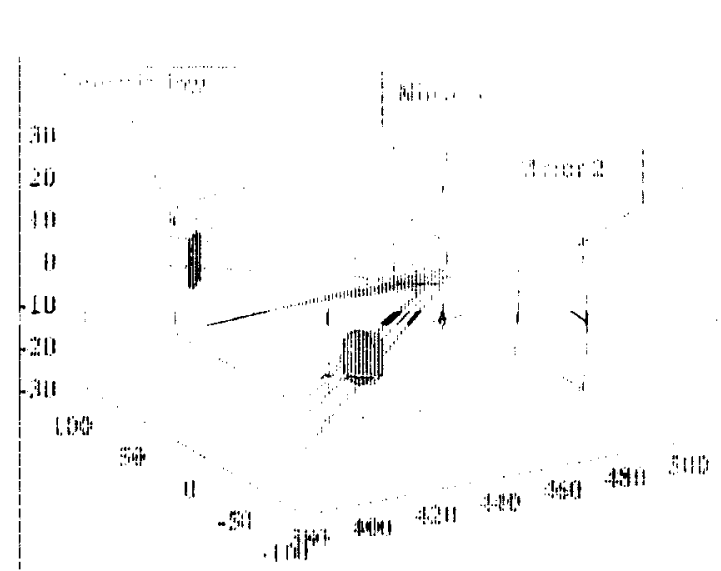

(a) Schematic of a new CC segment.

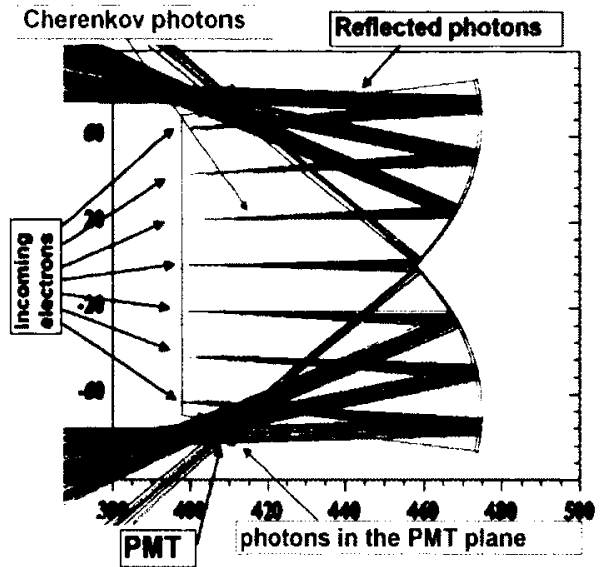

(b) Schematic of Cherenkov light reflections in a new $\mathrm{CC}$ unit.

FIG. 26. Schematic of a new CC segment showing the arrangements of the mirrors, PMTs and the light reflections (courtesy of INFN, Genova).

- Detection and primary triggering of electrons at energies above $0.5 \mathrm{GeV}$. The total energy deposited in the $\mathrm{EC}$ is available at the trigger level to reject minimum ionizing particles or to select a particular range of scattered electron energy.

- Detection of photons at energies above $0.2 \mathrm{GeV}$. Allowing $\pi^{0}$ and $\eta$ reconstruction from the measurement of their $2 \gamma$ decays.

- Detection of neutrons, with discrimination between photons and neutrons using TOF measurements [71].

In our experiment, DC, SC and EC counters were used in the standard CLAS configuration. The modifications for this experiment were only in the CC (see section 3.4.4), torus polarity (outbending for electrons), the Moeller shield (new one made of Tungsten which is denser than lead which was used previously), and the position of the target (at -100.93 $\mathrm{cm})$ relative to the CLAS center. 


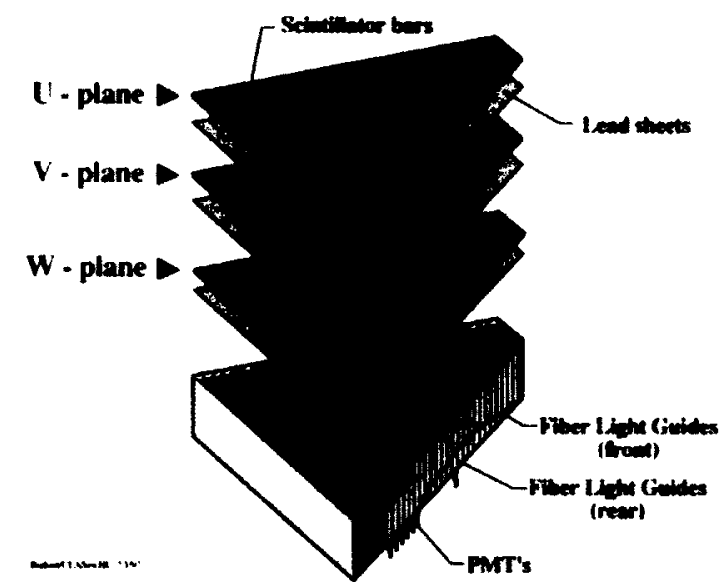

(a) EC Sandwich

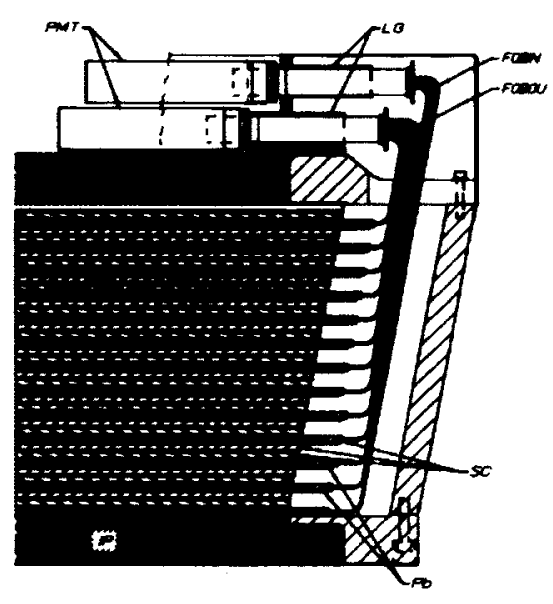

(b) EC Readout

FIG. 27. EC sandwich (left) and the readout (right)

\subsection{TRIGGER AND DATA ACQUISITION SYSTEM (DAQ)}

Each detector subsystem in Jefferson Lab has its own electronic modules ${ }^{8}$ to monitor its performance as well as to collect its signals for further analysis. A detector can produce signals due to a number of unwanted reasons such as the incidence of a cosmic radiation or the intrinsic electronic noise, and so not all signals are desired. There exists another electronic system - the trigger-system - whose job it is to determine whether a given set of detector signals constituted a desired physics event. The trigger acts as an interface between the detector subsystems and the final data-acquisition system (DAQ), which receives the desired signals and records them on disks in intended data formats for online as well as off-line analysis. The trigger helps minimize the dead-time of the detectors and also minimzes the resources required to process and store data.

A two-level trigger hierarchical system is generally used with CLAS to acquire the desired events. The level-1 (L1) trigger, which is dead-timeless and uses all prompt PMT signals within $90 \mathrm{~ns}$, controls the data acquisition through the front end electronics using a trigger supervisor (TS) module by providing a common start signal to the ADCs (Analogue to Digital Converters) and TDCs (Time to Digital Converters) and a delayed common stop

\footnotetext{
${ }^{8}$ Commercial (FASTBUS or VME) modules were used whenever possible, but custom modules with in-house designs were developed when commercial options were not available, or the application was so specialized that significant gains in performance or cost could be achieved [75].
} 
signal to the DC electronics. When a L1 trigger signal occurs, i.e. the event is accepted, further signal reception from CLAS is halted for $2 \mu$ s while the Level-2 trigger (L2) uses additional information from the acquired data to make the final decision on whether to reject the data and reset the front end electronics or convert and read out the data. If L2 okays an event, the conversion is initiated and the detector will not go live until all ADCs and TDCs have digitized and locally buffered their data, a process which typically takes $20 \mu \mathrm{s}$ [76]. In this experiment, only the Level-1 triggers requiring a coincidence between the signals (above some specified thresholds) in the EC and the new CC (from INFN) were used. Because the new $\mathrm{CC}$ was only in sector-6, no electron triggers came from the other five sectors, thus, basically using them only to record the other coincident particles from the accepted multi-particle final states. In addition to the normal production data, a few special "EC-only" data runs were also taken with no $\mathrm{CC}$ signals required in the event triggers. These special data sets together with normal data were used in estimating the (in)efficiency of the CC-detector.

After passing through the pre-trigger discriminators, all prompt PMT signals containing information about the hit locations in SC, and CC and deposited energy levels in EC are sent to a custom electronic system to make groupings and sums of them to generate a fast 62 bit signal, which, in turn, is sent as input to the L1 trigger in order to decide if a desired event has occurred. Using a three-stage pipelined memory lookup with a pipeline speed of $67 \mathrm{MHz}$, the input bit pattern is compared against preloaded patterns in memory tables which are programmed using a graphical software package called TIGRIS [75].

As soon as a Ll signal comes, the TS generates the gates for the detectors to allow their signals to be digitized in the 24 FASTBUS and VME crates stationed in the experimental hall, waits for conversion (by ADCs, and TDCs) of all crates to complete, and then places the event on the readout queue by sending all information to the 24 VME Readout Controllers (ROC1 to ROC24). (The readout happens asynchronously with conversion.) Fig(28) shows the overall schematic of the data data flow in the CLAS DAQ. The arrays of digitized values related to different detector components collected by the 24 ROCs are translated into tables with each data value (having a size of up to 16 bits) in it given a unique number to identify which component of the detector was responsible for the data. The tabulated data is then transferred via fast Ethernet cables to the CLAS online acquisition (CLON10) computer in the control room, with three primary processes - the Event Builder (EB), Event Transport (ET), and Event Recorder (ER) - running in it. The EB assembles the data pieces coming in the form of different tables to build the complete events, 
and then packages the complete event data by prefixing individual tables with headers to form "banks" and labelling the collection of banks by a run and event number, an event type, and the trigger bits which are put in a separate header bank. The packaged event is then passed to the ET managed shared memory (ET1) in the CLON10 which allows simultaneous access by various event producer or consumer processes running on the local or remote systems. The ER collects data from the ET1 and writes it in a single stream to a local array of magnetic RAID disks. When the disk is full, the data is transferred to a remote tape silo managed by the computer center a kilometer away ${ }^{9}$. Some events from ET1 are also sent to remote ET systems, e.g. ET2 and ET3, for the online monitoring purposes.

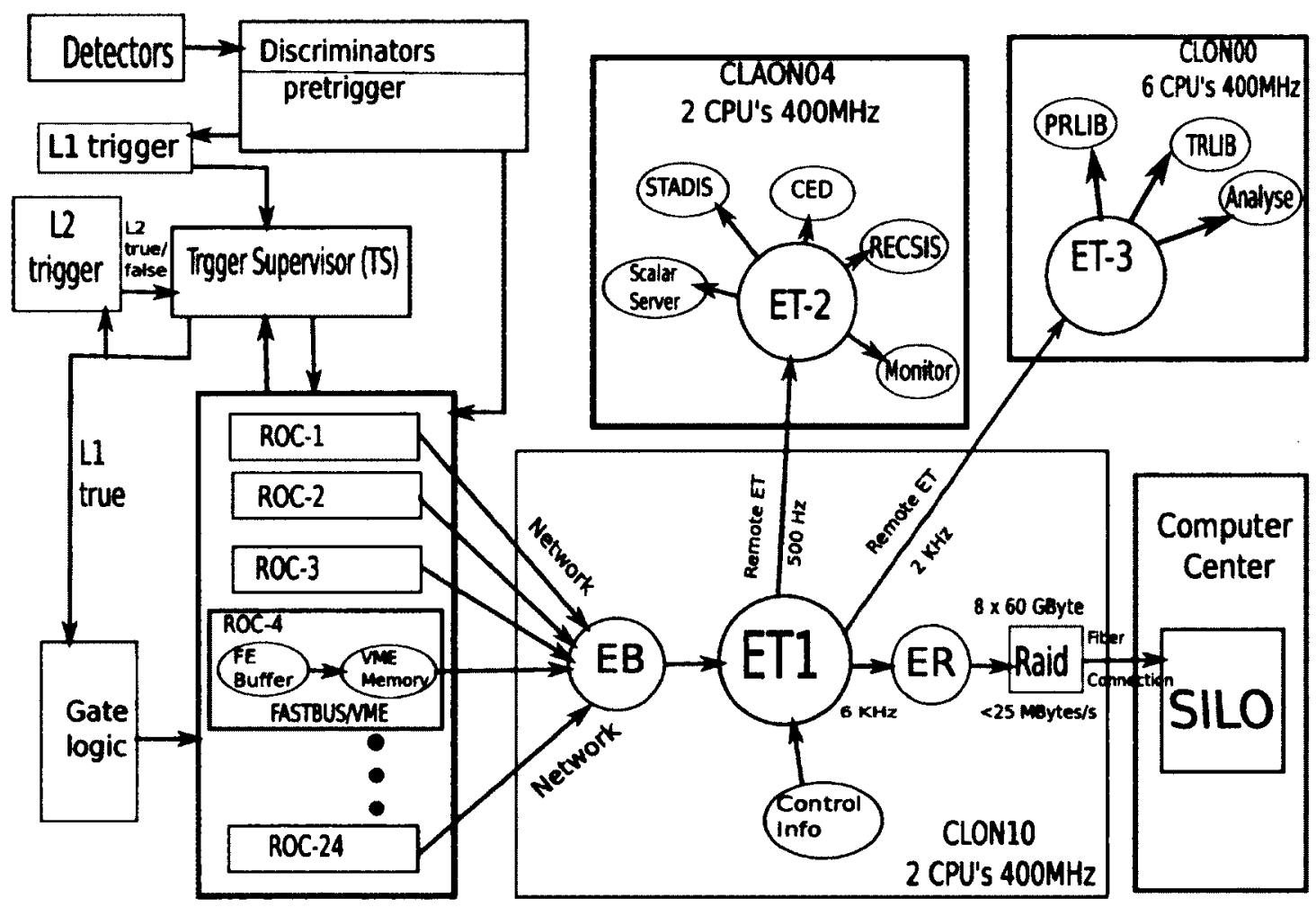

FIG. 28. An schematic of the data flow in the CLAS DAQ.

\footnotetext{
${ }^{9}$ Since the maximum tape writing speed is small (about $10 \mathrm{MByte} / \mathrm{s}$ ) [75], data transfers are performed in parallel, so that consecutive files may end up in different tape silos
} 


\section{Trigger bits}

Every reconstructed event in the BOS file has, in its HEVT bank, an integer variable called trigger bits, which represents a 16-bit binary number that carries trigger information about various detector components (the level-1 trigger bit variable in the ntuple-10 is named 'Ilbit'). An N-bit binary number is an array of $\mathrm{N}$ zeros and ones which represents the following:

$$
a_{1} 2^{0}+a_{2} 2^{1}+a_{3} 2^{2}+a_{4} 2^{3}+a_{5} 2^{4}+\ldots+a_{N} 2^{N-1}
$$

where, the coefficients $a_{n}$ are either 1 or 0 (i.e., the bit is either present or absent). For an example, if the trigger bits variable has the value of 41481 , it means that the bits $1,4,10$, 14 , and 16 are present and others absent because,

$$
41481=2^{0}+2^{3}+2^{10}+2^{13}+2^{15}
$$

Out of the 16 bits, the first six (1-6) indicate whether there was proper event trigger $(\mathrm{CC}+\mathrm{EC})$ in the sectors 1-6 respectively. The next two (7th and 8th) represent EC triggers only, with 8 representing a lower EC threshold than that normally used for event triggers. The next six bits (9-14) are left unused and the last two (15-16) both indicate (redundantly) the sign of the corresponding helicity bucket.

In general, only those events that have valid hits in at least one sector are good for inclusive analysis, so only events with at least one bit present out of 1-6 are kept and the rest is discarded. In fact, EG4 used only the 6th sector, so only events with the 6th bit present are useful for the final analysis. The bits 7 and 8 are useful for works such as pion background studies. The last two bits are eventually overriden by the modified variable read from the fixed helicity tables and, hence, go unused. 


\section{CHAPTER 4}

\section{DATA ANALYSIS}

As stated in previous chapters, the goal of this data analysis is to extract the spin structure function $g_{1}$ for the deuteron and evaluate its moments. We also saw (see Sec. 2.2) that the product $A_{1} F_{1}$, which is proportional to $\sigma_{T T}$, directly enters sum rules for the real photon point, which leads to the generalized GDH integral $\left(\bar{I}_{T} r\right)$ and the generalized forward spin polarizability $\left(\gamma_{0}\right)$ being expressed in terms of the first and third moments of the product $A_{1} F_{1}$. In view of that, we decided also to extract the product $A_{1} F_{1}$ using exactly the same procedure as for $g_{1}$.

The extraction of both $g_{1}$ and $A_{1} F_{1}$ depend directly on the measurement of the following polarized cross-section difference:

$$
\Delta \sigma_{\|}=\frac{d^{2} \sigma^{\downarrow \Uparrow}}{d \Omega d E^{\prime}}-\frac{d^{2} \sigma^{\uparrow \Uparrow}}{d \Omega d E^{\prime}}=\frac{1}{N_{t}} \cdot\left[\frac{N^{+}}{N_{e^{-}}^{+}}-\frac{N^{-}}{N_{e^{-}}^{-}}\right] \cdot \frac{1}{P_{b} P_{t}} \cdot \frac{1}{\Delta \Omega} \cdot \frac{1}{E_{\text {detector }}}
$$

where,

- $N_{t}=$ Number of deuteron nuclei in the target

- $N^{+/-}$: Number of scattered electrons (off deuteron only) for each helicity state $(+/-)$.

- $N_{e^{--}}^{+/-}:$Number of incident electrons for $+/$- helicity states

- $P_{b} P_{t}=$ Product of the beam and target polarizations

- $\Delta \Omega=\sin \theta \cdot \Delta \theta \cdot \Delta \phi:$ The solid angle for the given kinematic bin. This term includes the "detector acceptance".

- $E_{\text {detector }}$ accounts for the detector efficiencies

The data analysis to extract the physics quantities involves accurately measuring each of these quantities, either separately or in some combined form. To do so, the data must be properly reconstructed, calibrated and corrected to build all the scattering events during the 
experiment. Since the reconstructed events include a wide range of physical processes in addition to the electron-deuteron scattering process that we are interested in, proper event selection cuts must be applied. In this chapter, all these steps from the data reconstruction and calibration through the extraction of $g_{1}$ are described.

\subsection{EG4 RUNS}

The deuteron target part of the EG4 experiment ran for about a month in 2006, mostly with longitudinally polarized frozen $\mathrm{ND}_{3}$ as the target. In between these deuteron runs, some small amount of data was also collected on carbon-12 and empty cell targets, which are important in various auxiliary studies during the data analysis (such as their use in estimating nuclear background while developing momentum corrections, estimating the length of the target material or estimating unpolarized background). A total of 113 data runs (from run ID 51896 to 52040) were collected for the lower beam energy (1.3 GeV) and 221 runs (from 51593 to 51867 ) for the $2.0 \mathrm{GeV}$ case (with each run consisting of about $3.0 \times 10^{7}$ event triggers) [77]. Each run took about 2 hours and collected about 2 GB of data in raw format and saved as about 20-30 BOS files (see next section). With the combination of low beam energy and low scattering angle, low momentum transfers can be measured down to about $0.02 \mathrm{GeV}^{2}$ within the kinematic coverage of the resonance region $(1.08<W<2.0 \mathrm{GeV}$ )

\subsection{RAW DATA PROCESSING - CALIBRATION AND RECONSTRUCTION}

The raw data recorded by the CLAS DAQ system consist of ADC and TDC values registered by different components of the detector (in response to the passing of various particles through them) and also some beam related information such as Faraday Cup readings and beam helicity information. These data are collected and saved (by the DAQ system) in the fortran-77 based BOS format [78] which implements a dynamic memory management. In the BOS file, the data is organized into banks, with each bank carrying data belonging to a particular detector or some part of it. Each bank consists of two parts header and body. The body contains the actual collected data (such as the ADC and TDC values from detector components such as PMTs, in the case of raw data, or information such as energy and time in case of the reconstructed data), while the header contains some relational information - such as the bank identifier, the number of rows and columns of data in the bank and the location of the next bank. In the case of reconstructed data, in addition 
to the banks carrying detector specific reconstructed information such as the energy and time of the signals, more banks are added to the data file for storing high level information such as the number of particles detected in a given event, the four-momenta of each of the particles etc.

These raw data are next processed with another standard CLAS software package called RECSIS, which analyzes and combines the matching bits and pieces of the raw information (in the form of the TDC and ADC values from various detector components) to reconstruct particles and events that produced those signals. Such reconstruction produces output data that consist of event and particle IDs, particle positions and energies and momenta (in the lab frame CLAS coordinate system), and also some static particle properties such as charge and mass. The reconstruction program uses geometric parameters and calibration constants (from the CLAS database) for the detector to process and convert the raw data into the output form.

This iterative work of data reconstruction and detector calibration, which was a very computing intensive and time consuming, was done by R. De Vita - one of the EG4 collaborators from INFN, Genova, with good expertise on CLAS data reconstruction - soon after the data collection was completed (from 2006-2007).

The first part of the data processing is the detector calibration. In this phase, a small sample (about $10 \%$ ) of raw data (uniformly selected over the entire run period to ensure time stability verification) is chosen and the energy and time calibration constants are adjusted to give the correct behavior while constantly monitoring related variables. This is done separately for each run period to consider the different running conditions, the possibility of unwanted changes in hardware that may have occurred, as well as drift of detector response over time. This process of adjusting the calibration constants and reconstructing the data is repeated until a desired level of accuracy is reached. Once that level is reached, the calibration constants are "frozen" and the final reconstruction is done. The resulting output is saved in especial formats'. These saved data provided the starting point for our higher level data analysis as described in this dissertation. The details of the calibration and reconstruction process can be found in [62].

\subsection{HELICITY STATES}

As we saw from Eq. 102, the physics extraction depends on measurements of the number of events in the two (+/-) electron helicity states. The CEBAF accelerator provides

\footnotetext{
${ }^{1}$ Two especial data formats - BOS and ntuple (h10) - were used
} 


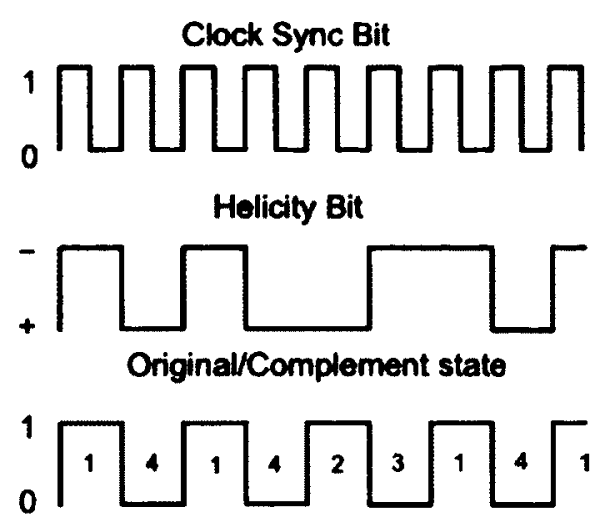

FIG. 29. Different data signals sent from the injector that monitor the helicity states of beam electrons. (Fig. courtesy of N. Guler [35] ).

the polarized electrons in closely and equally spaced bunches. These bunches are further grouped into "buckets" according to their helicity states, which are alternated pseudorandomly at the injector with a frequency of $30 \mathrm{~Hz}$. The information on the helicity state of each of the buckets and the total integrated charge contained in it is injected into the DAQ data stream immediately after the helicity flip. Using a combination of different types of sequence control signals sent from the injector (see Fig. 29), it is possible to determine which helicity state a particular event belonged to, which then can be used to label the helicity state of the event in the data stream, together with the total beam charge of the state.

\subsection{DATA QUALITY AND STABILITY CHECKS}

With an available set of good event/electron selection cuts, beam charge (measured by Faraday cup) normalized total event counts (sometimes also known as event "yield"), as well as polarization dependent differences, were calculated for each of the data files for all the runs and then plotted against the run number to study the data quality and stability as shown by Figs. 30, 31 and 32.

If nothing unusual happened or if the experimental conditions are not changed, then it is expected that the event yield as well as the count differences remain constant over time. Therefore, the graphs of these event counts plotted versus time or run number (which also roughly reflect the flow of time) should indicate the stability and quality of the data collected. For example, Fig. 30 shows such a total yield plot for all the data files from 
the $2.0 \mathrm{GeV}$ beam energy data set on deuteron target. We can see that these data runs display some features of instability over the full period of time, but stability over short time periods. For example, all the data with run numbers below about 51610 show significantly higher event yield than the runs after that run (possibly due to beam-target misalignment as indicated by raster magnet ADC values in Fig 32.

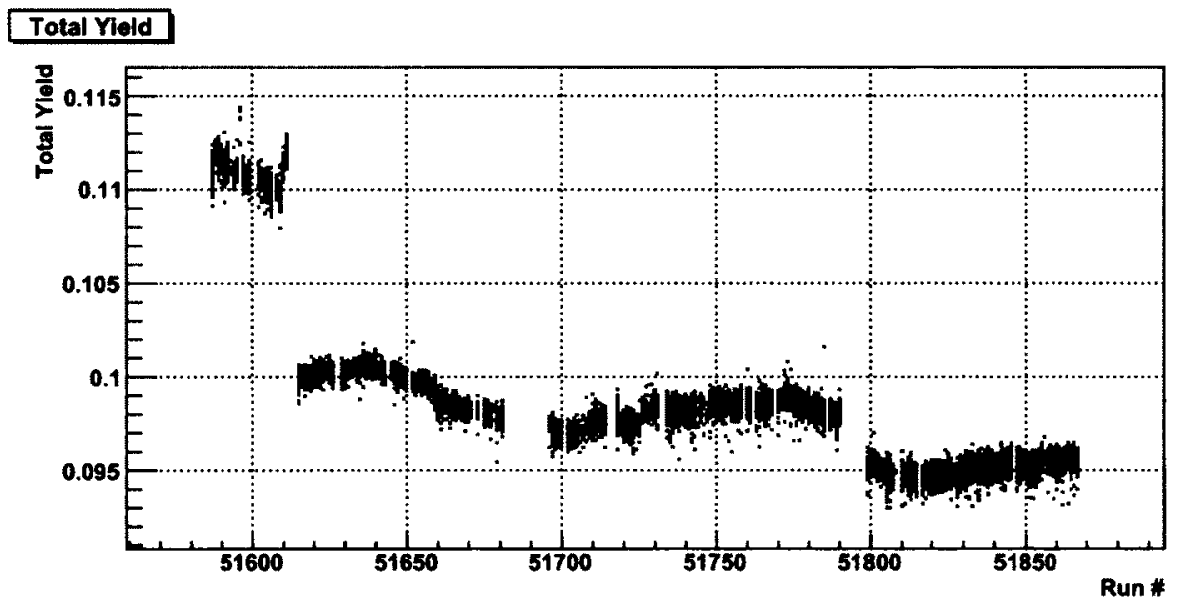

FIG. 30. Total normalized yield $\left(=\frac{N^{+}+N^{-}}{F C^{+}+F C^{-}}\right)$for $2.0 \mathrm{GeV} \mathrm{ND}$ runs.

Likewise, the stability of the polarized count differences in the elastic region $(0.9 \mathrm{GeV}$ $<W<1.0 \mathrm{GeV}$ ) as well as separately in the delta $(\Delta)$ resonance region were studied by plotting them versus the same run numbers (here the elastic and $\Delta$-resonance regions are considered separately, because the spin spin asymmetries in these two regions have opposite signs, which would have decreased the observed difference if combined. To further enhance the sensitivity of the observation, the difference of the count differences measured in the elastic and $\Delta$-resonance regions as given by

$$
\Delta N_{\text {elastic }}-\Delta N_{\Delta}=\frac{1}{P_{b} P_{t}}\left[\left(\frac{N^{+}}{F C^{+}}-\frac{N^{-}}{F C^{-}}\right)_{\text {elastic }}-\left(\frac{N^{+}}{F C^{+}}-\frac{N^{-}}{F C^{-}}\right)_{\Delta}\right]
$$

were plotted (see Fig. 31). It was observed that this elastic normalized count difference (which is what really matters to our analysis, in the end) was much more stable than the total yield. 


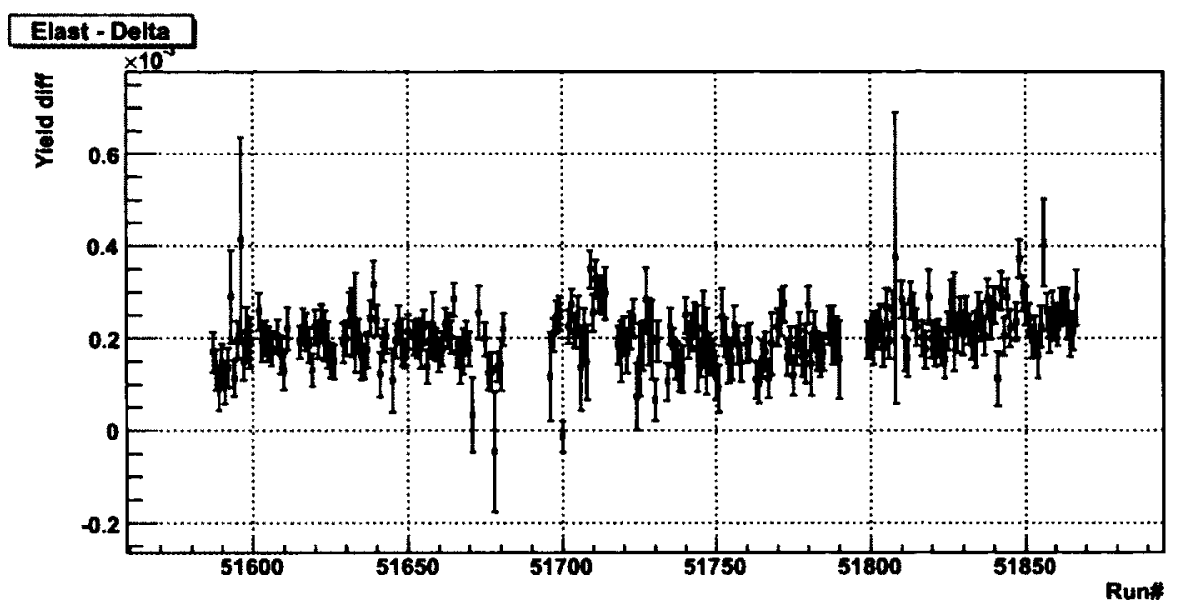

FIG. 31. Polarized yield differences (Eq. 103) normalized with $P_{b} P_{t}$ and BPM/F-cup for elastic peak minus that for the $\Delta$ peak for the $2.0 \mathrm{GeV} \mathrm{ND}_{3}$ runs.

The same was also repeated for the other variables such as the root-mean-square of the $A D C$ values (see Fig. 32) which carry information on the $X$ and $Y$ coordinates of the beam at the interaction vertex, thus their plots giving us somewhat more direct information on whether there was any misalignment between the beam and the target.

Based on the studies of these quality and stability plots, the data runs were divided into subgroups with each beam energy data set. In each subgroup, the data showed more stability than over the whole run period for the given beam energy. For example, in case of the $2.0 \mathrm{GeV}$ deuteron data, the runs were divided into four distinct sub groups corresponding to the four separate bands as seen in the Fig. 30. These subgroups were later treated and analyzed separately to get the corresponding normalized polarized count differences (with all data runs from each subgroup combined together). After the initial combination within the subgroups, they were again combined into the grand total by properly considering the half-wave-plate status, and the target polarization directions. 

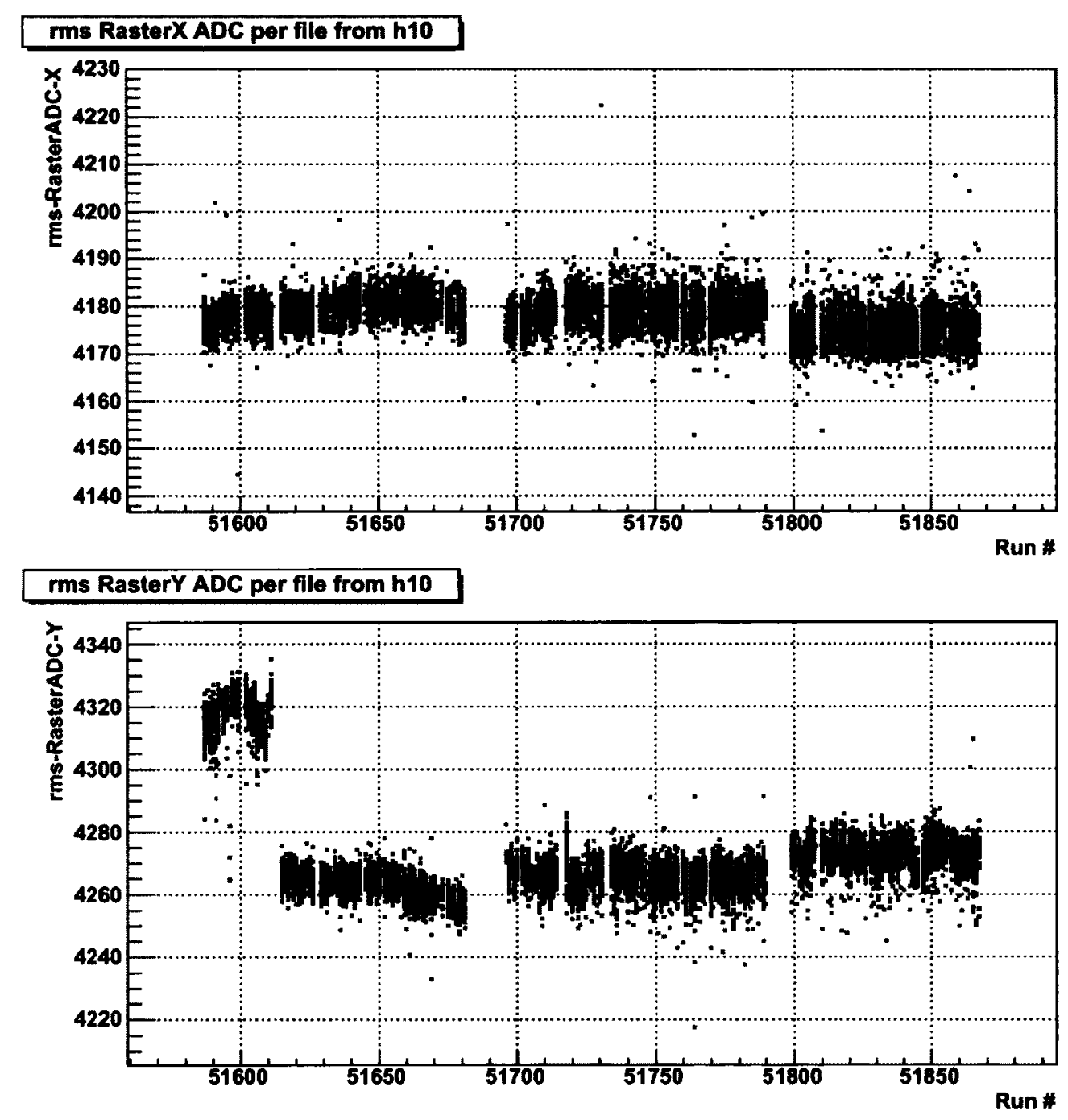

FIG. 32. Root-mean-square of the ADC values for the raster magnet currents in the directions $\mathrm{X}$ and $\mathrm{Y}$. The distributions show a larger raster size in the $\mathrm{y}$-direction for the first group of runs, indicating that the beam may have been hitting the edges and the walls of the target or other more dense structure support materials, thus explaining the higher total yield for the corresponding runs as shown by the Fig. 30. This does not affect our final analysis because these off-target materials are not polarized and, hence, do not contribute to the polarization dependent count difference $(\Delta N)$ used in the final analysis. 


\subsection{KINEMATIC CORRECTIONS}

Particle 4-momenta and event vertices as detected by CLAS and reconstructed by RECSIS are not accurate enough for various reasons. First, RECSIS does not take into account the fact that the beam is rastered in polarized target experiments. Next, any imperfections and mis-alignments of detectors and other components of the experimental set-up are not accounted for. Also, the torus field map is not known precisely. In addition, the effects of multiple-scattering and particle energy losses are not considered in RECSIS. Therefore, to get more accurate results from the data analysis, the data quality must be improved by applying various kinematic corrections. Following is the list of the corrections that were applied for analysis:

1. Incoming (beam) energy loss correction (due to ionization)

2. Raster correction

3. Drift chamber dependent momentum correction

4. Z-vertex correction

5. Solenoid axis tilt correction

6. Solenoid axis offset correction

7. Multiple scattering correction

8. Outgoing energy loss correction (due to ionization after scattering)

The first correction listed above considers the loss of beam energy due to atomic collisions before the actual nuclear scattering takes place. A good estimate for this loss is $2 \mathrm{MeV}[63,79]$, which is subtracted from the nominal beam energy. This correction is applied during the analysis whenever the beam energy is involved, and therefore it is not included in the correction package described below.

\subsubsection{RASTER CORRECTION}

The polarized electron beam coming from CEBAF to Hall $\mathrm{B}$ is rastered in polarized target experiments. This is done to minimize radiation damage (depolarizing effects) to the polarized target and also to make maximum use of the target material (effective beam size increases and, therefore, the overall volume of exposed target increases). The beam 
is periodically spiraled covering a circular region of the target cross-section by using two raster magnets - one for the horizontal $(\mathrm{X})$ direction and the other for the vertical direction (Y). The currents driving the two magnets are continuously recorded by analog-to-digital converters (ADCs).

Projection onto $x-y$ CLAS plane ( $z$-axis = beam axis points into plane of figure)

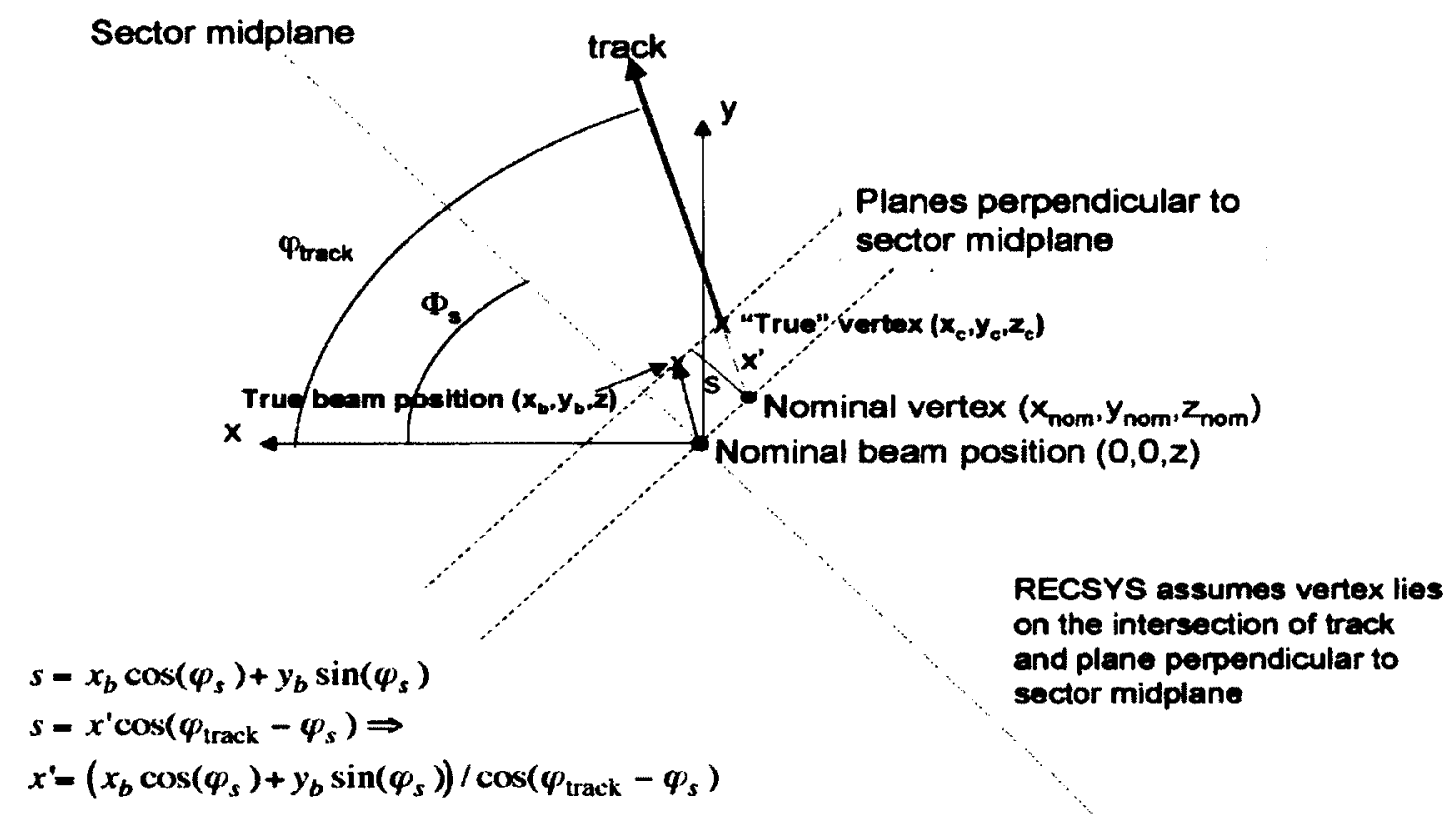

FIG. 33. Raster correction geometry illustration (Figure courtesy of S. Kuhn)

The ADC values thus recorded can be translated to the coordinates $(x, y)$ of the exact beam position at the target. The values of $x, y$ can then be used to make corrections to the original track by RECSIS (which assumes $x$ and $y$ were zero), allowing better $z$-vertex and azimuthal angle $(\phi)$ reconstruction. The better $z$-vertex reconstruction allows better selection of events from the target proper, rejecting events from upstream and downstream windows (especially for particles at small angles), and can also be used to reduce accidental coincidences in multi-particle final states (or to look for offset decays such as from $\Lambda$ ). Correction of $\phi$ improves missing mass resolution for multi-particle final states which is very important in exclusive channel analysis. In addition, plotting a two-dimensional 
histogram of events as a function of the raster information $\mathrm{x}$ and $\mathrm{y}$, one can look for missteered beam that might have hit the target cup edges.

\section{Projection onto scattering plane}

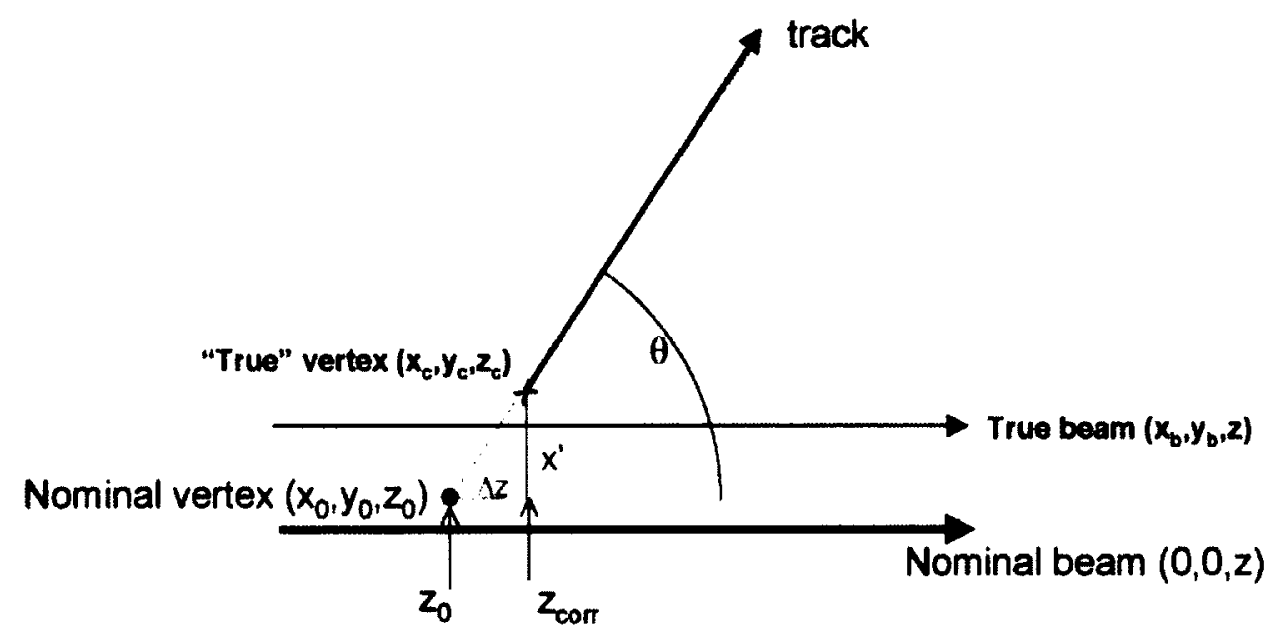

$$
z_{c o r r}=z_{0}+\Delta z=z_{0}+x^{\prime} / \tan \theta
$$

FIG. 34. Raster correction geometry illustration (Figure courtesy - S. Kuhn)

A procedure was developed by $\mathrm{P}$. Bosted et al. [80] to translate the raster ADC values into the beam coordinates $x, y$ and then use them to improve the $z$-vertex and $\phi$ reconstruction. This procedure was successfully applied in previous CLAS experiments and EG4 has also embraced it to do the needed raster correction.

In short, the procedure for this correction is as follows:

1. Translates raster-ADC values to beam coordinates $x$ and $y$.

2. Corrects the event vertex z-coordinate (represented as $v_{z}$ in the data).

3. Corrects the azimuthal angle $\phi$ of each particle in the event.

This correction is applied before the momentum correction. So, the partially corrected $\phi$ and $v_{z}$ will be a part of the input fed into the next stage of the kinematic correction 
which, henceforth, will be termed "momentum correction".

\section{Procedure to translate ADCs to centimeters}

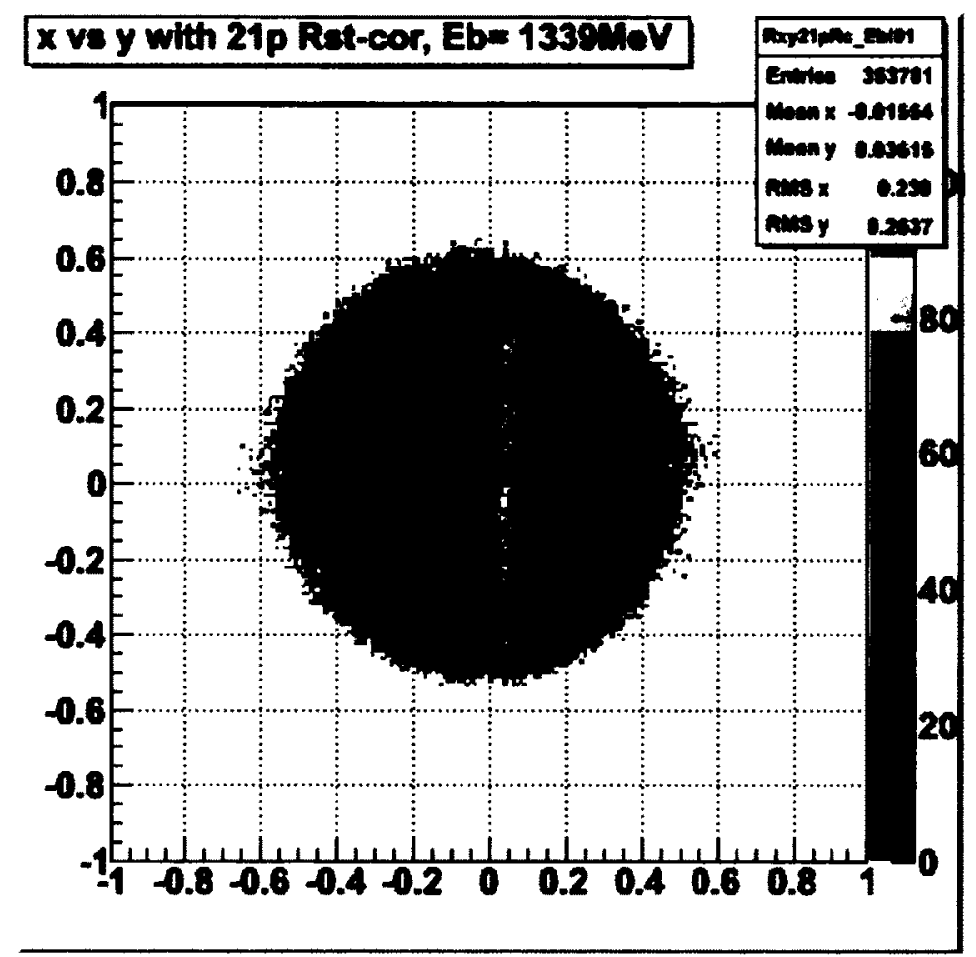

FIG. 35. Beam coordinates $\mathrm{x}$ and $\mathrm{y}$ calculated with the raster correction procedure.

The procedure assumes that a linear relation holds between the raster currents and the beam coordinates $\mathrm{x}$ and $\mathrm{y}$ (displacements in $\mathrm{cm}$ produced by the field of the currents) as follows:

$$
\begin{aligned}
& x=\left(X_{a d c}-X_{o f f s e t}\right) C_{x}, \\
& y=\left(Y_{a d c}-Y_{o f f s e t}\right) C_{y},
\end{aligned}
$$

where, $X_{o f f s e t}, Y_{o f f s e t}, C_{x}$, and $C_{y}$ are the parameters to be determined by the procedure. These parameters are determined by selecting reasonably well reconstructed events each 
consisting of more than one charged particles tracks originating reasonably close to the nominal target center $\left(\mathrm{v}_{z} \approx-101.0 \mathrm{~cm}\right)$ and using them in TMinuit (ROOT Minuit program) to minimize the $\chi^{2}$, defined as

$$
\chi^{2}=\sum_{i=1}^{N}\left(\left(z_{c o r r}\right)_{i}-z_{o}\right)^{2},
$$

where $z_{0}$ is the 5 th parameter that defines the center of the target and is to be determined from the minimization. Likewise, $z_{\text {corr }}$ is the trial value of the corrected $z$-vertex (a function of trial values of the first four fit parameters, as will be evident below). TMinuit will give us those values of the parameters which gives the $\chi^{2}$ a minimum value.

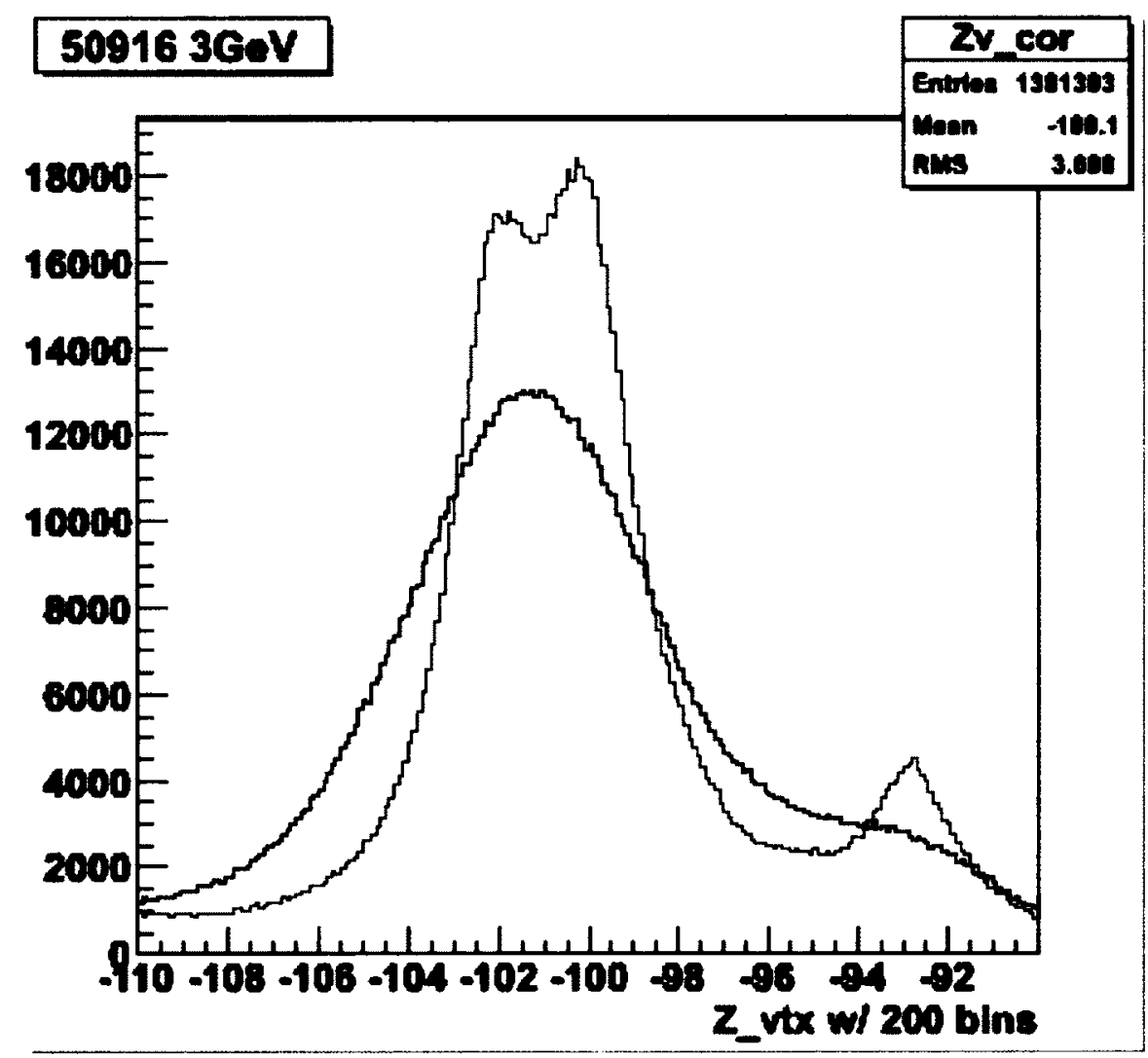

FIG. 36. Vertex Z-coordinates (in $\mathrm{cm}$ ) of scattered electrons from an $3.0 \mathrm{GeV}$ empty-celltarget run before (black) and after (red) the raster corrections. It is clear that the correction improves the resolution, thus revealing the positions of the empty target cells (the first two peaks near $-101.0 \mathrm{~cm}$ ) and the heat shield (around $-93.0 \mathrm{~cm}$ ).

From a simple geometry consideration (as illustrated in Figs. 33 and 34), an expression 
for correction to the z-vertex in terms of $x, y$ and angles of a particle track is arrived at as follows:

$$
z_{\text {corr }}=z_{\text {recsis }}+x^{\prime} / \tan (\theta)
$$

where $z_{\text {recsis }}$, and $z_{\text {corr }}$ are the $z$-vertex measured by the tracking code and the rastercorrected z-vertex respectively, and

$$
x^{\prime}=\left(x \cos \phi_{s}+y \sin \phi_{s}\right) / \cos \left(\phi-\phi_{s}\right)
$$

is the distance in $\mathrm{cm}$ along the track length that was not considered in tracking (because the tracking code assumes that the track started from $x=0, y=0) ; \phi_{s}$ is the sector angle defined as the azimuthal angle of the sector mid-plane (equal to $(s-1.60$ degrees, where $s$ is the sector number from 1 to 6 ), and $\phi$ is the azimuthal angle of the particle (in the lab-coordinate system) defined as $\phi=\arctan (c y / c x)$, where $c x$ and $c y$ are the $\mathrm{x}-$ and $\mathrm{y}-$ direction cosines of the track.

Due to the difference of the actual track length (through the $50 \mathrm{kG}$ magnetic field of the target) from what is assumed by the tracking software, the azimuthal angle $\phi$ is reconstructed incorrectly. The angle $\phi$ can now be corrected by adding a correction term $-50 q\left(x^{\prime} / 100\right) / 33.356 / p_{t}$ to the reconstructed value $\phi_{\text {recsis }}$ as follows:

$$
\phi_{c o r r}=\phi_{\text {recsis }}-50 q\left(x^{\prime} / 100\right) / 33.356 / p_{t}
$$

where $\phi_{\text {recsis }}$ and $\phi_{c o r r}$ are the reconstructed and corrected values of $\phi$ respectively, $q$ is the particle charge in units of $e$, the factor 50 is the target field expressed in $\mathrm{kG}$, the factor 100 is to convert the unit $\mathrm{cm}$ of $x^{\prime}$ to $\mathrm{m}$, the factor 33.356 is the inverse speed of light in the appropriate units and $p_{t}=p \sin (\theta)$ is the particle's transverse momentum expressed in $\mathrm{GeV}[80]$.

For our analysis, all the four parameters $X_{o f f s e t}, Y_{o f f s e t}, C_{x}$, and $C_{y}$ were determined separately for each beam energy by selecting a set of good electrons and using the method of $\chi^{2}$ minimization (see Eq. 105). With the parameters known, we can use Eqs. 104a and 104 to convert the $\mathrm{X}$ - and $\mathrm{Y}$ - ADC values into beam positions (at the target location) in centimeters as shown in Fig. 35 for $1.3 \mathrm{GeV}$ data. Likewise, $v z$ and $\phi$ can be corrected by calculating the correction terms $x^{\prime} / \tan (\theta)$ and $-50 q\left(x^{\prime} / 100\right) / 33.356 / p_{t}$ and adding them to the respective reconstructed values (see Eqs. 106, 108). For example, Fig. 36 shows the distribution of electron $\mathrm{Z}$-vertex distribution (from $3 \mathrm{GeV}$ proton data) before and after the corrections. It is evident from the figure that the corrections improves the resolution 
as expected in addition to shifting (towards left) the average position of the distribution by some amount.

\subsubsection{DRIFT CHAMBER (DC) DEPENDENT MOMENTUM CORRECTION}

Different DC related factors contribute to the biggest part of the systematic deviations of particle momenta as reconstructed by RECSIS. The drift chambers could be misaligned relative to their nominal positions or the survey results that is used by RECSIS could be inaccurate or out-of-date. The effects of physical deformations (due to thermal and stress distortions) of the chamber including wire-sag, incorrect wire positions may not have bee incorporated properly. The torus field map used by the reconstruction software may not have been accurate and complete enough [81]. To address issues like these, a general approach as described in [81] which makes corrections to $p$ and $\theta$ was followed to develop the corrections.

The ratio of the correction to the magnitude of the momentum could be expressed as:

$$
\frac{\Delta p}{p}=\text { Pcorr } 1+\text { Pcorr } 2+\text { PatchCorr }
$$

where,

$$
\begin{gathered}
P \operatorname{corr} 1=\left((E+F \phi) \frac{\cos \theta}{\sin \phi}+(G+H \phi) \sin \theta\right) \frac{p}{q B_{\text {torus }}} \\
P \operatorname{corr} 2=(J \cos \theta+K \sin \theta)+(M \cos \theta+N \sin \theta) \phi \\
\text { PatchCorr }=0.02\left(P+\left(Q+R \frac{\phi_{\text {deg }}}{30^{\circ}}\right)\left(\frac{10^{\circ}}{\theta_{\text {deg }}}\right)^{3}\right)
\end{gathered}
$$

The quantity $B_{t o r}$ stands for $\int B_{\perp} d l$ along the track length multiplied by the speed of light in the units of $\mathrm{m} / \mathrm{ns}(\mathrm{c}=0.29979 \mathrm{~m} / \mathrm{ns})$ and is given by

$$
\begin{aligned}
& B_{t o r}=0.76 \frac{I_{t o r} \sin ^{2}(4 \theta)}{3375 \theta / \mathrm{rad}} \quad \text { for } \quad \theta<\frac{\pi}{8} \\
& B_{t o r}=0.76 \frac{I_{t o r}}{3375 \theta / \mathrm{rad}} \quad \text { for } \theta>\frac{\pi}{8}
\end{aligned}
$$


In all these equations, sector number, $\theta, \phi, \theta_{d e g}$, and $\phi_{d e g}$ come from the angle information measured at DC1. The direction cosine variables tll_cx, tll_cy, tll_cz (from pass 1 ntuples) are used to derive these quantities. $\mathrm{C}++$ standard functions $\operatorname{acos}()$ and $\operatorname{atan} 2()$ are used to evaluate $\theta, \phi$ (w.r.t the sector mid plane).

All these total of eleven unknown parameters were determined separately by fitting above mentioned momentum offsets (in combination with ionization energy loss correction for electrons) to the correction function given by the Eq. 109.

Unlike for sector- 6 , both $\mathrm{p}$ - and $\theta$ were subjected to correction if a given particle track was detected by the drift-chamber in any of the other 5 sectors. This time, the PatchCorr component was not considered in the expression (Eq. 109) for p-correction. On the other hand, following expression was used to parameterize the correction to the polar angle $\theta$.

$$
\Delta \theta=(A+B \phi) \frac{\cos \theta}{\cos \phi}+(C+D \phi) \sin \theta
$$

A total of 12 ( 8 for $p$-correction and 4 for $\theta$ correction) parameters for each of these five sectors were determined (from a fit procedure to be described below) to account for the DC contribution to the corrections.

\subsubsection{SOLENOID CORRECTION}

If the axis of the target solenoid field is not aligned exactly along the beam line, then the $\phi$ reconstruction is skewed. To correct for that, the following changes are made to the reconstructed angles:

$$
\begin{aligned}
& c x_{\text {true }}=c x_{i n i}+a / p \\
& c y_{\text {true }}=c y_{\text {ini }}+b / p
\end{aligned}
$$

where $c x$ and $c y$ are the $x$ - and $y$-direction cosines, $p$ is the particle momentum and and $\mathrm{b}$ are the parameters to be determined by the fit (described in 4.5.6). It's clear that $c x$ and $c y$ and therefore $\phi=\arctan (c y / c x)$ is changed by this part of the correction.

The target field may also have an overall displacement or offset w.r.t the beam line and so the following correction to the angles is used in addition to the other corrections: 


$$
\begin{aligned}
& \phi_{\text {true }}=\phi_{\text {ini }}+q B_{\text {solenoid }} \frac{S \cos \phi_{\text {ini }}-T \sin \phi_{i n i}}{p \sin \theta_{\text {ini }}} \\
& \theta_{\text {true }}=\theta_{\text {ini }}+q B_{\text {solenoid }} \frac{U \cos \phi_{i n i}-V \sin \phi_{i n i}}{p}
\end{aligned}
$$

Here, $\mathrm{S}, \mathrm{T}, \mathrm{U}$ and $\mathrm{V}$ are the additional parameters to be determined by the method of $\chi^{2}$ minimization (see Sec. 4.5.6) for the overall correction.

RECSIS evaluates the vertex assuming that it lies on the intersection of the track and the plane perpendicular to the sector mid-plane that contains the beam axis [82]. So, RECSIS backtracks the DC-reconstructed particle track and finds the point where the track meets this plane to determine the vertex. As a consequence, while doing the raster correction, we correct $Z$ in addition to $\phi$. Since the track itself is subject to further corrections even after the raster correction, the vertex should also be corrected further. The following expression is used to further correct the z-component of the vertex.

$$
z_{\text {true }}=z_{r s t}+Y \frac{\theta-\theta_{\text {ini }}}{\sin ^{2} \theta}
$$

where $\theta_{i n i}$ is the polar angle (in radians) at the start, $\theta$ is the one after all the previous corrections and ' $\mathrm{Y}$ ' is the new fitting parameter to be determined whose physical meaning is the distance from the vertex to the first region of DC (about $150 \mathrm{~cm}$ ) [83].

\subsubsection{MULTIPLE SCATTERING CORRECTION}

As a particle travels away from the reaction vertex, it encounters additional scattering centers (within the target material as well as outside) before being registered by the CLAS detector. That means, even if the detectors record the track perfectly, its angles most likely would not be the same as the ones at the vertex. Since the vertex position is reconstructed based on the angle information, the reconstructed vertex would also be shifted from the real one by some amount (see Fig. 37).

This effect is common in all CLAS experiments and a simulation study (using GSIM (see Sec. gsim)) ) on this issue was done by the collaborators of the CLAS EGIb and EGldves experiments [84]. The study indicated that tracks could be corrected for this effect if there are multiple track in an event, with the corrections to the angles given by simple parameterized formulas. These corrections are based on the assumption that the real z-vertex of all the coincident particles in an event is close to the average z-vertex for 


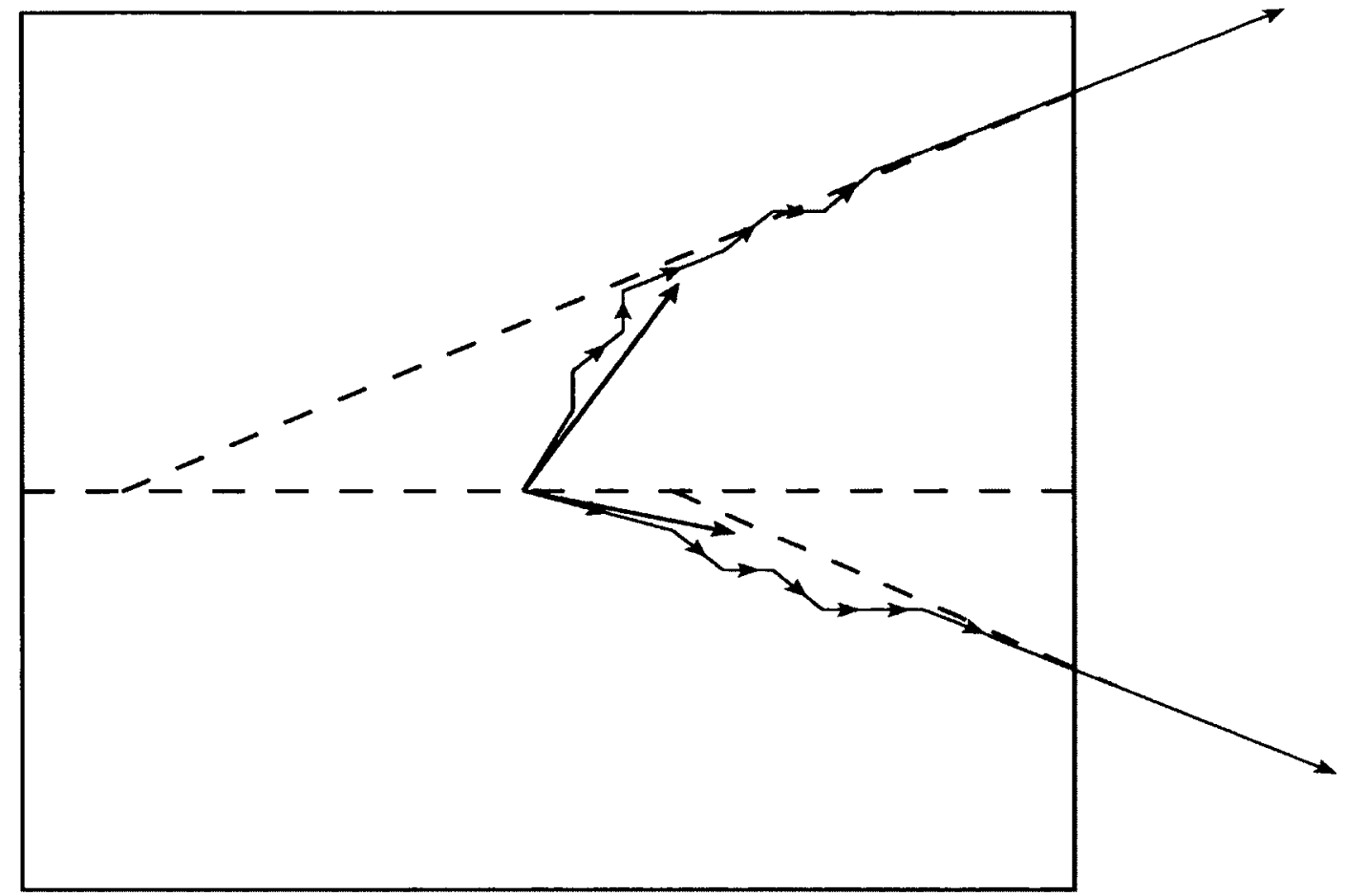

FIG. 37. An exaggerated figure showing the effect of multiple scattering.

the event, and that the angles should be modified accordingly. The average z-vertex is calculated as:

$$
z_{\text {ave }}=\langle z\rangle=\frac{\sum z_{\text {part }} / z_{\text {res }}^{2}}{\sum 1 / z_{\text {res }}^{2}}
$$

where the sums are over all the well-identified charged particles from the event, $z_{\text {part }}$ is the $\mathrm{z}$ position of each of the particles (with all of the previous corrections applied), and $z_{r e s}$ is the resolution in $\mathrm{z}$ which is given by

$$
z_{\text {res }}=\frac{0.1}{p_{\perp} \beta}
$$

where $p_{\perp}$ is the transverse component of the 3 -momentum given by $p \sin \theta$ and $\beta$ is the particle speed in the units of the speed of light.

After finding the weighted average of the $z$-vertex, all the particles from the event are 'forced' to originate from that vertex and the angles are corrected as follows:

$$
\begin{gathered}
\theta_{\text {true }}=\theta_{\text {ini }}-\left(z-z_{\text {ave }}\right)\left(L \theta_{\text {ini }}+M / p\right) \\
\phi_{\text {true }}=\phi_{\text {ini }}-q\left(z-z_{\text {ove }}\right) N / p
\end{gathered}
$$


where $\mathrm{L}, \mathrm{M}$, and $\mathrm{N}$ are the three more parameters to be determined from the fit (see Sec. 4.5.6).

\subsubsection{OUTGOING IONIZATION LOSS CORRECTION}

After all the previous corrections are made, the energy of each of the particles is calculated as $E=\sqrt{p^{2}+m_{\text {rest }}^{2}}$ and a correction for ionization loss is added to it: $E_{\text {cor }}=E+\Delta E$ with $\Delta E=\frac{d E}{d X} \tau$ where the factor $\tau$ is the total effective mass thickness traversed by the particle and

$$
d E / d X \approx 2.8 \mathrm{MeV} /\left(\mathrm{g} \mathrm{cm}^{-2}\right) \text { for electrons }
$$

and, for hadrons [85]

$$
d E / d X \approx 0.307 \times \frac{0.5}{\beta^{2}}\left(\ln \left(2.0 \times 511.0 \frac{\beta^{2} \gamma^{2}}{0.090}\right)-\beta^{2}\right) \mathrm{MeV}
$$

which is an approximation of the Bethe-Block formula [85]:

$$
-\frac{1}{\rho} \frac{d E}{d x}=4 \pi N_{a} r_{e}^{2} m_{e} c^{2} \frac{Z}{A} \frac{1}{\beta^{2}}\left(\ln \left(\frac{2 m_{e} c^{2} \gamma^{2} \beta^{2}}{I}\right)-\beta^{2}\right)
$$

This quantity is calculated as follows:

- $\tau=\tau_{\|} / \cos \theta \quad$ if $\theta<=\pi / 4$

- $\tau=\tau_{\|} / \cos (\pi / 4) \quad$ if $\theta>\pi / 4$

where $\tau_{\|}$is calculated as:

- $\tau_{\|}=\Delta z \times 0.6+0.4$ if $\Delta z>0.0$ and $\Delta z<1.0$

- $\tau_{\|}=0.6+0.4$ if $\Delta z \geq 1.0$

- $\tau_{\|}=0.4$ if $\Delta z \leq 0.0$

with $\Delta z=z_{\text {target_center }}-z_{\text {ave }}+L_{\text {target }} / 2=\left(-101.0 \mathrm{~cm}-z_{\text {ave }}+0.5\right) \mathrm{cm}$ being the physical distance (along the target length) traveled by the particle through the polarized target material (e.g. the $E G 4 N_{3}$ target has length $1.0 \mathrm{~cm}$ and is positioned at $\mathrm{z}=-101.0 \mathrm{~cm}$ ). The factor 0.6 is the effective mass thickness of $\mathrm{ND}_{3}$ (density of $\mathrm{ND}_{3}\left(\sim 1 \mathrm{~g} / \mathrm{cm}^{3}\right)$ multiplied by the packing fraction which is roughly 0.6 [61], whereas 0.4 is the sum of the mass thicknesses of $\mathrm{He}(\sim 0.3)$ and that of window foils $(\sim 0.1)[35]$. 
Using the ionization loss corrected energy and the rest mass of the particle, momentum is recalculated as $p_{c o r}=\sqrt{E_{c o r}^{2}-m^{2}}$ (where $m$ is the mass of the particle). Finally, this new $\mathrm{p}$ is used along with the previously corrected angles to evaluate the three cartesian components $p_{x}, p_{y}$ and $p_{z}$ of the momentum.

\subsubsection{PROCEDURE TO DETERMINE THE PARAMETERS}

As is clear from above sections, all together, there are 81 parameters to be determined for the various corrections. These are:

- 11 parameters for 6 th sector DC dependent momentum correction (corresponding to Eqs. (110), (111), and (112))

- $60(=12 \times 5)$ parameters for the same type of correction corresponding to other five sectors (corresponding to Eqs. (110), (111) and (115)).

- one parameter for the z-vertex correction (corresponding to Eq. (118))

- 6 parameters for solenoid tilt and offset corrections (corresponding to Eqs. (116) and (117))

- 3 parameters for the multiple-scattering correction (corresponding to Eq. (121))

Out of these, the first eleven parameters for the sector- 6 correction are determined from one procedure and the rest are determined from a different procedure.

\section{Procedure to determine the first 11 parameters}

The procedure involved dividing the covered kinematic space into a number of bins, finding in them the magnitude of shifts of the inclusive elastic peaks w.r.t. the expected position and use that to fit to a function to get an analytical expression for the correction. The following angular bins were used:

- Six $\theta_{d c 1}$ bins: $(0,8),(8,10),(10,12),(12,15),(15,20),(20,30)$ degrees

- Five $\phi_{d c 1}$ bins: $(-10,-6),(-6,-2),(-2,2),(2,6),(6,10)$ degrees

where the angles used are the ones measured at the first drift chamber and $\phi_{d c 1}$ is measured w.r.t the sector mid-plane (thus the maximum range allowed is $(-30.0,30.0)$ ). 


$$
E_{\text {elastic }}^{\prime}=\frac{E_{\text {beam }}}{1+\frac{2 E_{\text {beam }}}{M_{p}} \sin ^{2}\left(\theta_{e} / 2\right)}
$$

In each of these kinematic bins, the quantity $\Delta E=E_{\text {elastic }}^{\prime}-p$ is histogrammed for both $\mathrm{NH}_{3}$ and $\mathrm{C}_{12}$ data separately. Next, the carbon histogram is cross-normalized with the ammonia histogram and subtracted from the latter one to remove the nuclear background. The difference gives histogram for the elastic events (as shown by the dashed green histogram in Fig. 38. A Gaussian fit to the extracted elastic histogram gives the position and width of the distribution. The offset or shift of average position of the peak with respect to the expected $\Delta E=0$ gives us the needed correction on energy $E \approx p$ for the electron. This process is repeated for all of the bins listed above and the corresponding $\Delta_{E}$ offsets or the corrections are determined for each of them. Finally, these values of corrections for different average values of $\theta_{d c 1}$ and $\phi_{d c 1}$ are used into Eq. 109 and then used in the $\chi^{2}$-minimization based on four momentum conservation (as described below) in order to determine the 11 fit parameters.

\section{Procedure to determine the rest of the parameters}

This procedure uses the technique of $\chi^{2}$-minimization, where the $\chi^{2}$ is constructed from the 4-momentum conservation requirement in exclusive reactions for which sufficient statistics is available. Two types of events (from $\mathrm{NH}_{3}$ target runs) in which all particles in the final states are detected are chosen for this purpose, so that both higher and lower momenta are covered. Fully reconstructed elastic events $p\left(e, e^{\prime} p\right)$ are used to cover higher momenta and exclusive $p\left(e, e^{\prime} p \pi^{+} \pi^{-}\right)$events are used to cover lower momenta for different types of hadrons. The $\chi^{2}$ to be minimized is calculated as follows:

$$
\chi^{2}=\chi_{p-m i s s}^{2}+\chi_{W}^{2}+\chi_{z}^{2}+\chi_{p a r}^{2}
$$

where,

$$
\begin{gathered}
\chi_{p-\text { miss }}^{2}=\sum_{\text {all events }}\left(\frac{E^{2}(\text { miss })+p_{z}^{2}(m i s s)}{(0.020 G e V)^{2}}+\frac{p_{x}^{2}(m i s s)+p_{y}^{2}(m i s s)}{(0.014 G e V)^{2}}\right) \\
\chi_{W}^{2}=\sum_{\text {ep }- \text { events }}\left(\frac{\left(W-M_{p}\right)^{2}}{(0.020 G e V)^{2}}\right) \\
\chi_{z}^{2}=\sum_{\text {all particles }}\left(\frac{\left(z_{\text {cor }}-z_{\text {ave }}\right)^{2}}{z_{\text {res }}^{2}}\right)
\end{gathered}
$$




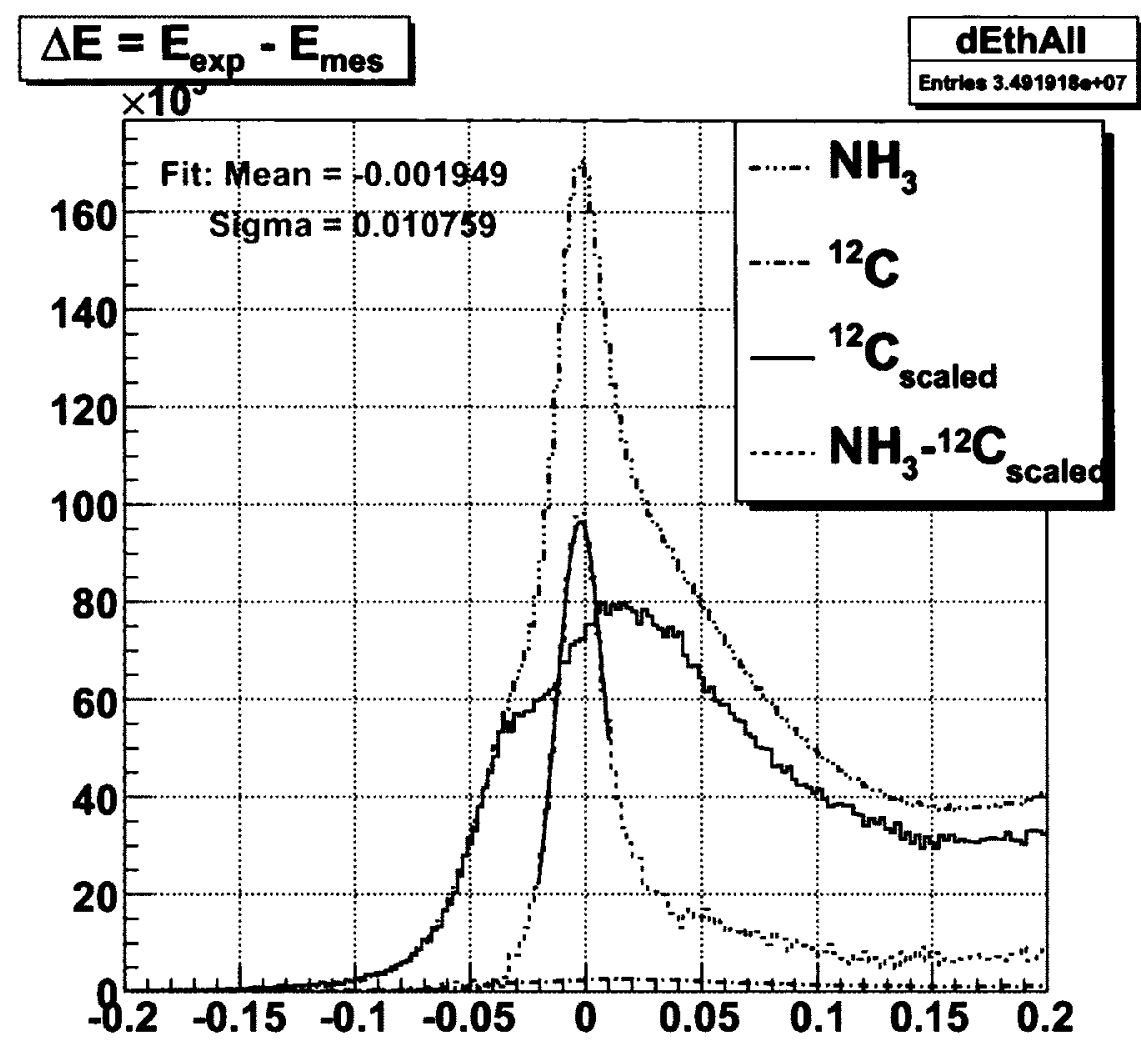

FIG. 38. Plots showing background removal from the $\Delta E$ counts from $\mathrm{NH}_{3}$ (shown by " $\mathrm{NH}_{3}$ " line) data (by subtracting cross-normalized counts from ${ }^{12} \mathrm{C}$ data (shown by

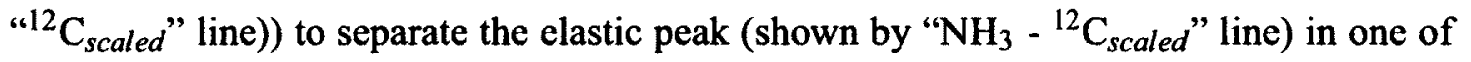
the kinematic bins, thereby getting the momentum offset for that bin.

$$
\chi_{\text {par }}^{2}=\sum_{\text {all parameters }}\left(\frac{\text { par }^{2}}{\sigma_{\text {par }}^{2}}\right)
$$

where $z_{c o r}$ and $z_{\text {ave }}$ are evaluated using Eq. 118 and 119 respectively after applying the DC dependent corrections.

\section{Event Selection}

For all events, the usual fiducial, preliminary vertex and electron ID cuts are applied (see later). Protons and pions are selected using time-of-flight (TOF) cuts, in addition to other simple common cuts. Finally, cuts on all four components of missing 4-momenta are applied to exclude events where not all produced particles were detected or where there were accidental co-incidences. These missing momentum cuts also serve to suppress nuclear 
background (from various sources such as from ${ }^{15} \mathrm{~N}$ nuclei in the ammonia target, from target windows etc.). The cuts used in the initial data skimming required that each of the four missing components be less than $0.1 \mathrm{GeV}$. Later during the fit, tighter cuts (up to 0.03 $\mathrm{GeV}$ for the $\mathrm{x}$ - and $\mathrm{y}$-components and up to 0.04 for $\mathrm{z}$ - and $\mathrm{E}$-components) were used. The cut on $E_{\text {miss }}$ also serves to remove events where the radiative (internal or external) loss in electron energy could be large enough to skew the momentum corrections. For elastic events, the three more cuts (on $W, \Delta \theta$, and $\Delta \phi$ ) were applied.

After the desired sample of the two types of exclusive events were selected, raster correction was applied to each of the particles from the sample, modifying/correcting the $z$-vertex and the azimuthal angle $\phi$ by some degree. Then the sample was subjected to the above mentioned $\chi^{2}$-minimization to optimize all the remaining free parameters for our momentum and angle corrections.

Once the minimization were complete, all the unknown parameters were determined and they were used to apply the corrections to $p$ and $\theta$. For example, Figs. 39, 40 and 41 show the effects of corrections on various quantities in different type of events. We can see that the corrections have not only shifted and improved the positions of various distributions but also improved their resolutions (narrowed distributions relative to the distributions before the corrections). 

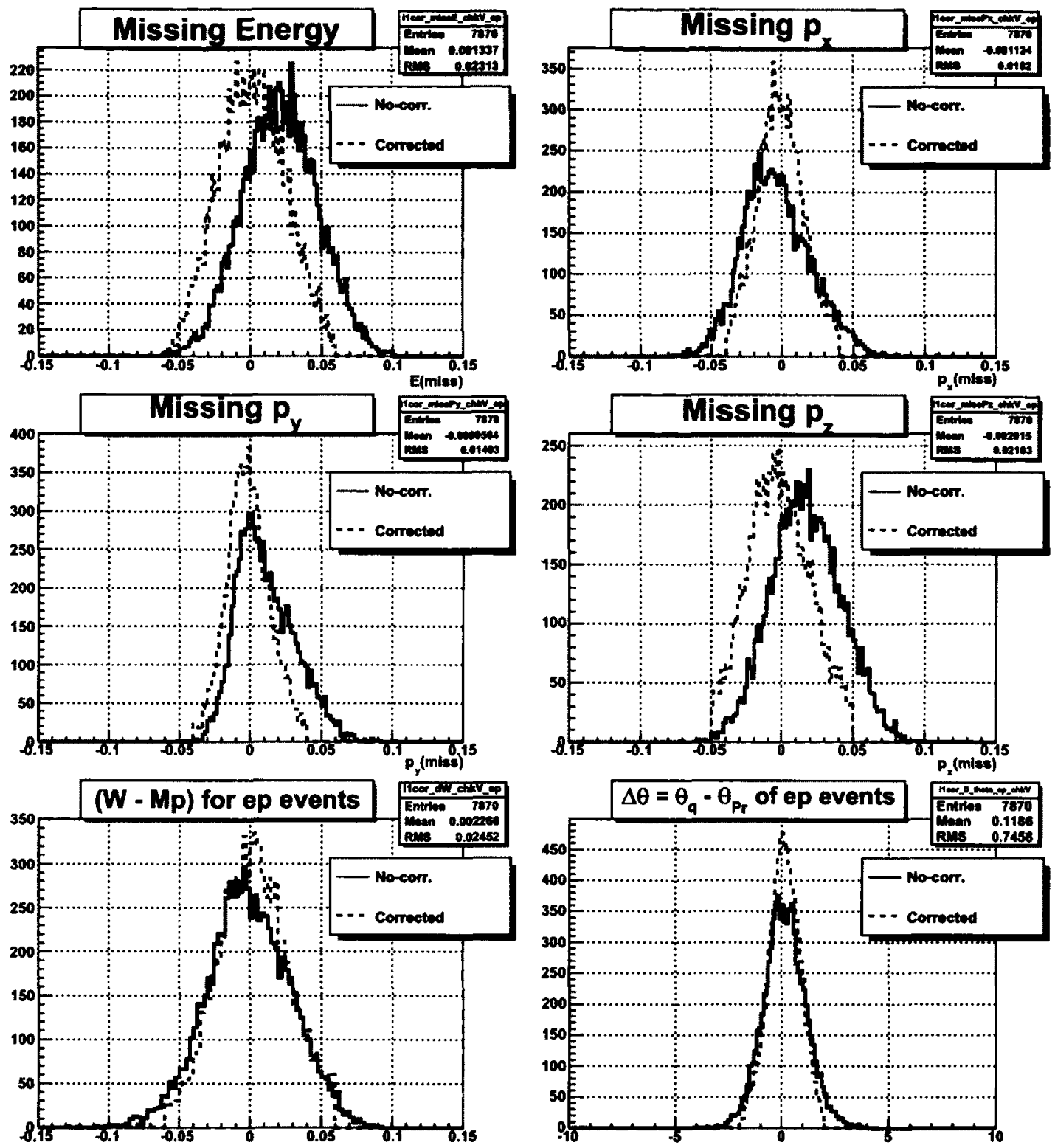

FIG. 39. Effects of kinematic corrections on ep-elastic events. In the 6 panels the distributions of missing energy, missing momentum components $\left(p_{x}, p_{y}\right.$, and $\left.p_{z}\right)$, the difference $\Delta W=W-M_{p}$ and $\Delta \theta=\theta_{q}-\theta_{p}$ of ep-elastic events respectively. The distributions before the corrections are shown by black continuous lines and the ones after the corrections are shown by the red dotted lines. Here, $M_{p}$ is the proton mass in GeV. Likewise, $\theta_{q}$ and $\theta_{p}$ are the expected and measured angles of the recoil proton (or the exchanged virtual photon) respectively. 

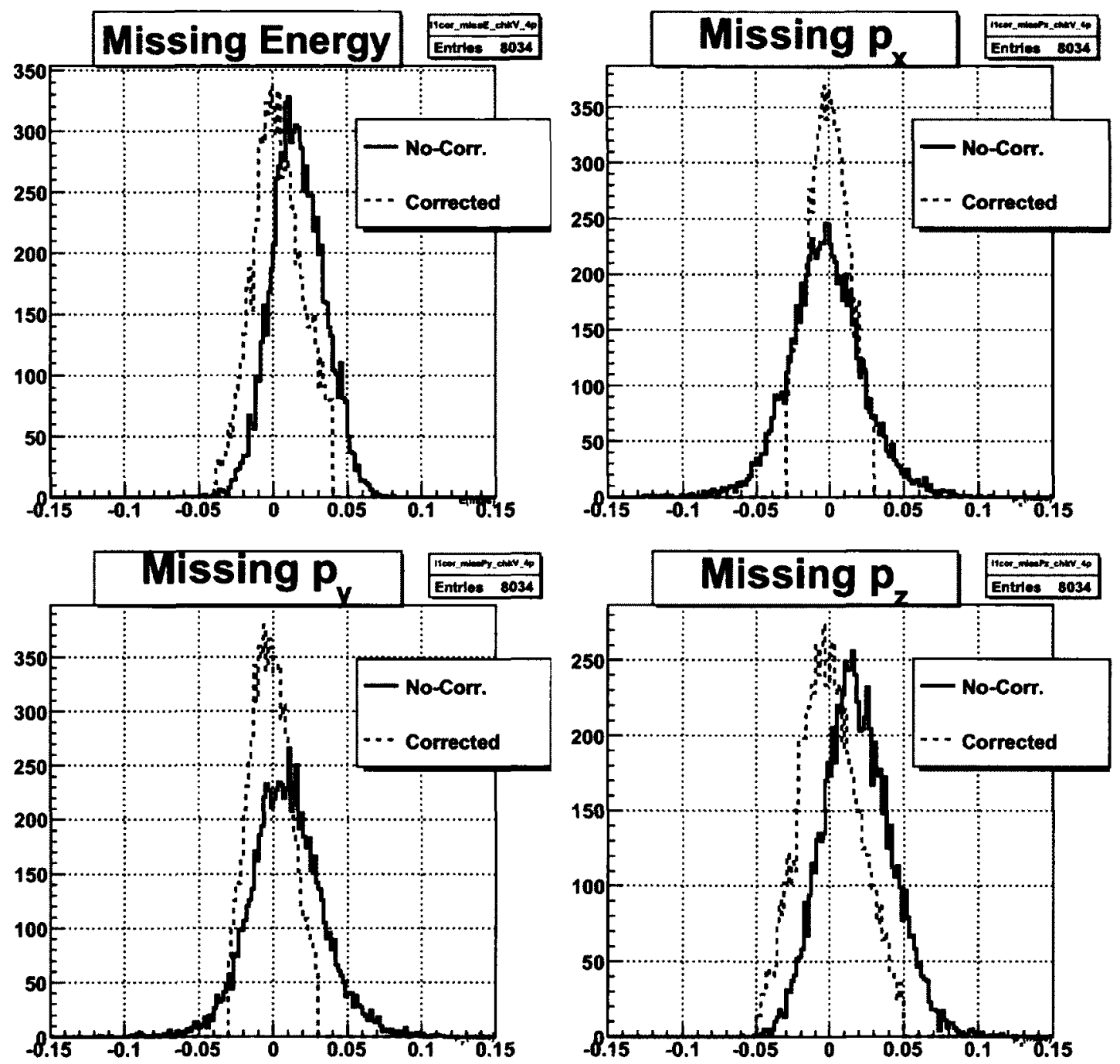

FIG. 40. Effects of kinematic corrections on ep2pi i.e. $p\left(e, e^{\prime} \pi^{+} \pi^{-}\right) X$ events. The four panels show the distributions of missing energy, and the three components of missing momenta for the events before (black) and after (red) the corrections. 


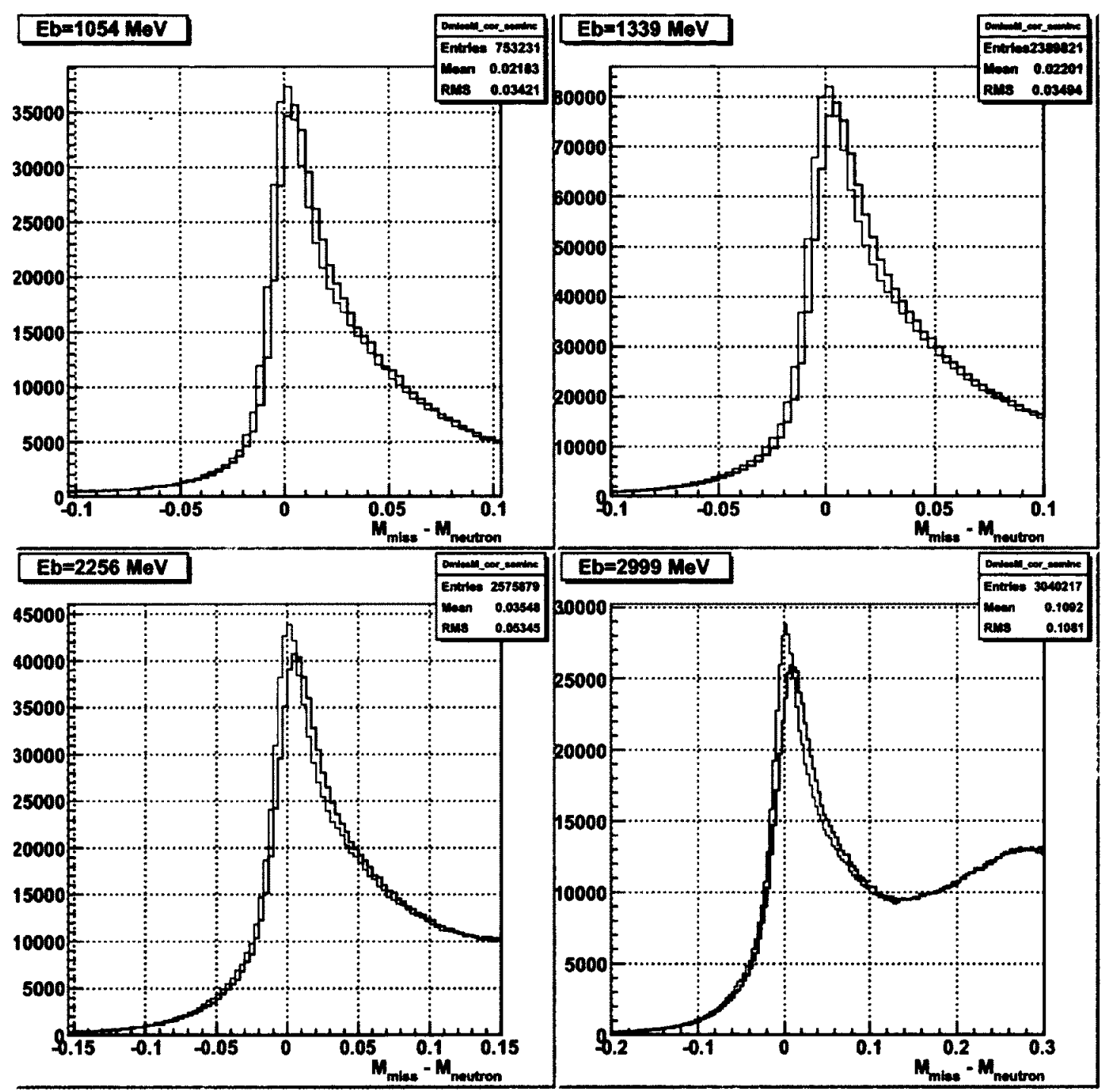

FIG. 41. Missing mass (minus the neutron mass) calculated before (black) and after (red) the kinematic corrections of semi-inclusive events of type $p\left(e, e^{\prime} \pi^{+} X\right)$ from four different $E_{b}$ run sets. 


\subsection{EVENT SELECTION CUTS}

In CLAS electron-scattering experiments, the scattered electron defines the timing of each event. In addition, in inclusive measurements, the scattered electron is the only particle to be detected and measured. So, it is particularly important to make sure that electrons are well measured and properly identified and are not contaminated with other misidentified particles such as negative pions $\left(\pi^{-}\right)$or lost by being misidentified as something else, thus affecting the accurate measurement of cross sections. In particular, $\pi^{-}$and electrons give rather similar detector signals and, therefore, are difficult to discriminate in some kinematic regions. In each event the electron candidate is the negative track that triggered the event. The trigger condition is ensured by choosing the first entry in the event and also requiring that the track has hit matches in $\mathrm{CC}, \mathrm{DC}, \mathrm{EC}$ and SC and is also time-based (positive DC status word in DCPB).

All four layers of detectors are important in identifying electrons. For example, tracking by DC decides the charge of a candidate, SC records the time of flight, which is important in the time-matching criteria as mentioned below. The following list shows criteria/cuts defining a good electron starting from a candidate electron.

In addition to the electron ID cuts, we also make further cuts to select only those events that originated from the polarized target and also only those that were detected within the fiducial region of the detector. In other words, one may divide the cuts into two categories - electron ID (or good electron) cuts and good event cuts. Following are the cuts used to select good electrons and good events:

\section{Good Electron Cuts}

(a) Cut on Particle charge: $q=-1$

(b) Detector Status cuts:

i. DC status: dc $>0$; dc_part $>0$

ii. SC status: $s c>0$; $s c_{-}$part $>0$

iii. EC status: ec $>0$; ec_part $>0$

iv. CC status: $c c>0$; $c$ _.part $>0$

(For simulated data, all of the above except those on $\mathrm{CC}$ variables are used.)

(c) Electromagnetic Calorimeter Cuts (see Sec. 4.6.1) 
(d) Osipenko cuts Cuts on $\mathrm{CC}$ angle $\theta, \phi$ and time matching between $\mathrm{CC}$ and other detectors. (see Sec. 4.6.2)

(e) Cut on Minimum Number of Photoelectrons (see Sec. 4.6.3)

\section{Good Event Cuts}

(a) Cut on Minimum number of particles detected and reconstructed in the event: gpart $>0$

(b) Minimum/Maximum Momentum cuts (see Sec. 4.6.4)

(c) Sector cut dc_sect $=6$; cc_sect $=6$ (to select electrons from the sector where the low momentum Cherenkov detector was installed)

(d) Scattering Vertex-Z cuts (see Sec. 4.6.5)

(e) Fiducial Cuts (see Sec. 4.6.6)

Data analysis method of this thesis relies on comparing the experimental data with a data set produced from a Monte-Carlo simulation that was as realistic as possible. The simulation process involves first the simulation of the physics process of inclusive electron scattering, then simulation of the CLAS detector response when the scattered electrons passed through it and finally reconstructing the events from the simulated detector responses using the same reconstruction software as used for the real data. So, we also have to analyze the simulated data in the same way as the experimental data requiring similar event selection cuts of their own. In the ideal situation, all cuts would be the same for both types of data. But, despite our efforts, we could not make our simulation match with our experimental data to the expected level - mainly due to some previously unseen issues with the reconstruction software (RECSIS). So, some of the data selection cuts are defined separately for the two cases and sometimes even for different $Q^{2}$ bins (to make sure we have the same fractions of events in corresponding kinematic bins for

\subsubsection{ELECTROMAGNETIC CALORIMETER CUTS}

The EC cuts basically consist of three different cuts applied together. One of these is on the sampling fraction (which is the fraction of the energy deposited in the calorimeter), another on the energy fraction deposited in the inner part of the calorimeter and the last is based on the correlation between the inner and outer energies recorded by the calorimeter. 


\section{Cuts on sampling fraction}

While moving through the $\mathrm{EC}$, charged pions are minimum ionizing particles in the momentum range detectable by CLAS. On the other hand, each electron deposits its total energy $E_{t o t}$ in the $\mathrm{EC}^{2}$ by producing electromagnetic showers $\left(E_{t o t} \approx p\right.$ for electrons that have high energies). Therefore, the sampling fraction $E_{t o t} / p$ should be independent of the momentum for electrons (in reality there is a slight dependence).

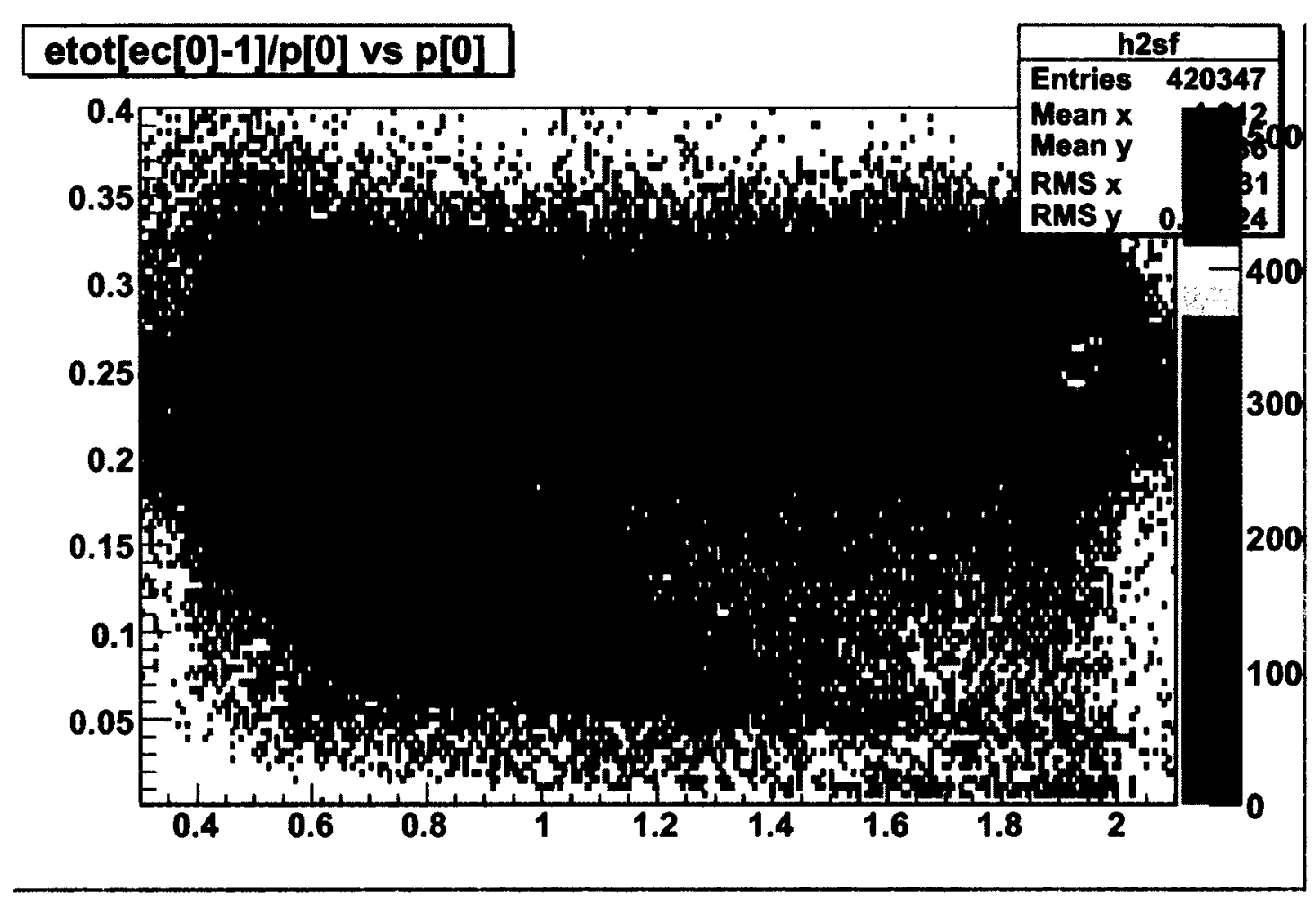

FIG. 42. An example of the cut on the EC sampling fraction ( $2.0 \mathrm{GeV}$ data). The plots shows the distribution of the sampling fraction (in Y-axis) plotted against the particle momentum (in $\mathrm{X}$-axis). The brighter stripe above about 0.2 in the energy fraction are due to the electrons whereas those below are the pions.

For the EC in CLAS, the electron sampling fraction is about 0.25 and pions give signals that are mostly below 0.2 as is evident in Fig. 42 or others that follow. Therefore, usually a

\footnotetext{
${ }^{2}$ Because some of the deposited energy is in the lead part of the EC rather than the scintillator, only a fraction of th electron energy is detected in the EC.
} 
universal lower cut of 0.2 is chosen to reject most of the pions without significantly losing good electrons. In our experiment, with its low beam energy, even less pions are produced and the electron peaks are even cleaner in lower kinematic bins as can be seen in the low $Q^{2}$ bins of Fig. 43. Therefore, in order to have fewer good electrons rejected, the sampling fraction cut was relaxed to 0.15 for the first fourteen of our $Q^{2}$ bins and 0.2 was kept for the higher bins.

In case of the corresponding simulation data, however, the cuts were not that simple because the EC performance simulation does not match the experimentally observed data well. As we can notice by comparing figures 43 and 44 , the mean positions and widths (represented by the $\sigma$ of a Gaussian fit) of the sampling fraction in different $Q^{2}$ bins are not exactly the same for data and simulation, which means that if we were to use the same rigid cuts of 0.15 or 0.2 , then we would have different fractions of events selected for simulated compared to experimental data. To avoid this situation, $Q^{2}$-bin dependent cuts were determined for the simulation based on the Gaussian fit parameters of both data and simulation as well as the above mentioned cuts used on the data. In a given $Q^{2}$ bin of simulated data, the cut on the left side of the electron peak is chosen that is at the same distance in terms of its $\sigma$ from its peak as the cut values 0.15 or 0.2 are from the peak in the experimental data in terms of its own $\sigma$. For example, suppose we are considering one of the first fourteen $Q^{2}$ bins, and $\mu_{\text {exp }}, \sigma_{\text {exp }}, \mu_{\text {sim }}$, and $\sigma_{\text {sim }}$ are the Gaussian fit parameters for the electron peaks in the experimental data and simulation respectively, then the cut on the simulation would be equal to $\mu_{\text {sim }}-\frac{\mu_{\text {exp }}-0.15}{\sigma_{\text {exp }}} \times \sigma_{\text {sim }}$.

In short, only two numbers 0.15 and 0.2 define the cuts on the experimental data, but the cuts for simulation data are all different, yet they are at the same relative distance from the electron peaks as in the experimental data and, therefore, include about the same fraction of good electrons.

\section{Cuts on $E_{\text {in }}$}

Pions, which do not shower and are minimum ionizing particles in the momentum range detected in CLAS, deposit only a (small) amount of energy in the inner part of the EC independent of their momentum. When $E_{\text {in }}$ is histogrammed, the tiny pion signal peak at about 0.03 clearly stands out from the huge electron sample with little overlap in between. So, a universal cut of $E_{\text {in }}=0.06$ on both data and simulation (as shown by figures 45,46 and 47) safely rejects most of the pions from the electron candidate sample. 

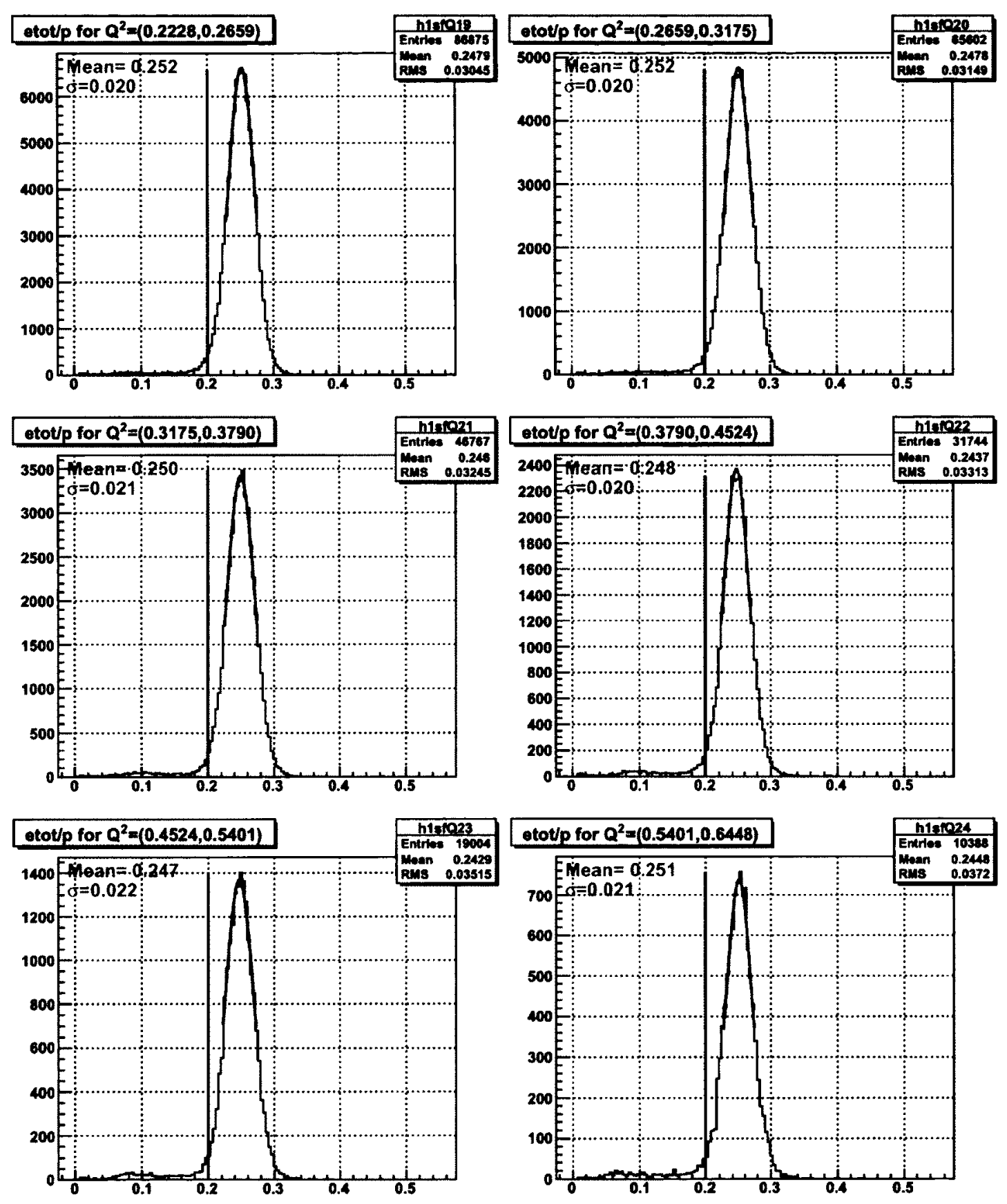

FIG. 43. The $Q^{2}$ dependent cuts on the EC sampling fraction for $2.0 \mathrm{GeV}$ experimental data. Events below the red lines are rejected. 

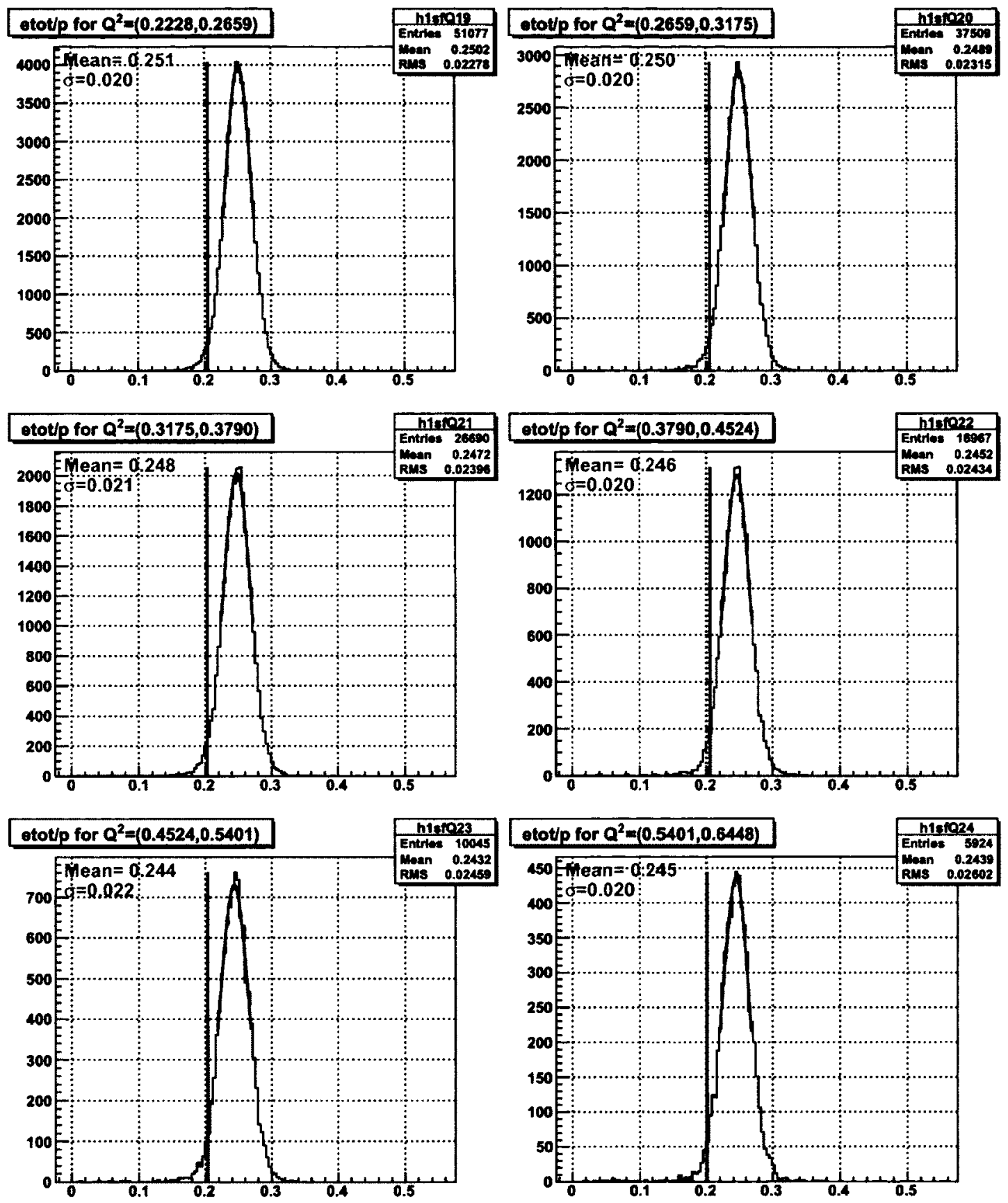

FIG. 44. The $Q^{2}$ dependent cuts on the EC sampling fraction for $2.0 \mathrm{GeV}$ simulation data. Events below the red lines are rejected. 


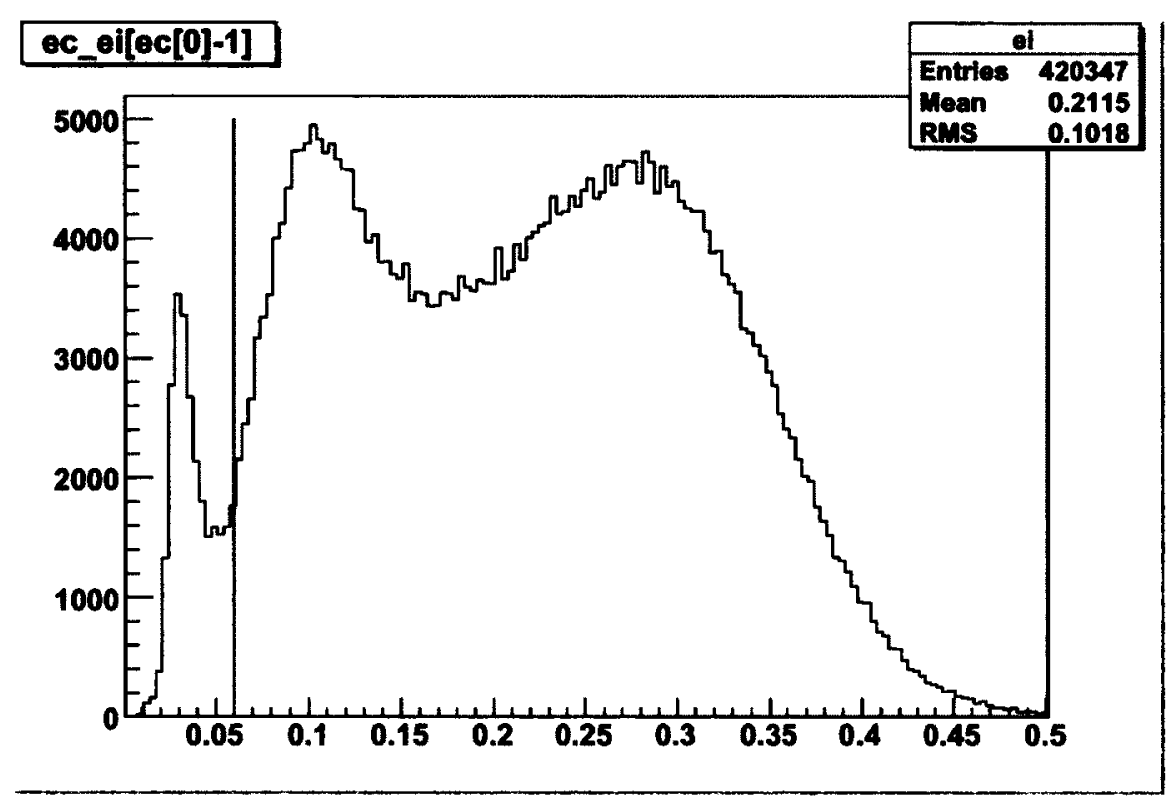

FIG. 45. Energy deposited (GeV) in the inner EC and the cut (red line) used to reject pions (seen as a peak at about $0.03 \mathrm{GeV}$ ) from a sample of electron candidates of $2.0 \mathrm{GeV}$ data.

\section{Cuts on $E_{\text {out }}$}

In addition to the two EC-cuts above, one more cut based on the correlation between ECouter and EC-inner (as shown by fig. 48) was used which helps further to clean up the electron sample.

\subsubsection{OSIPENKO (CC GEOMETRY AND TIME MATCHING) CUTS}

As discussed in chapter 3 the new EG4 dedicated CC is made up of 11 modules each consisting of a pair of mirrors and PMTs. The segments are placed along the CLAS polar angle covering 15 to 45 degrees, i.e., the segments are at different polar angular positions. During normal operation, the PMTs of these segments produce noise that is equivalent to that produced by one photo-electron passing through it. As a result, when a noise pulse in the $\mathrm{CC}$ and a pion track measured by DC coincides within the trigger window of the CLAS detector, the track gets registered as an electron candidate by the event reconstruction program, thus contributing to the contamination of electron candidates with the misidentified pion tracks. In fact, this turns out to be the biggest source of pion contamination. In order to minimize such contamination and help better identify electrons from pions, CC 

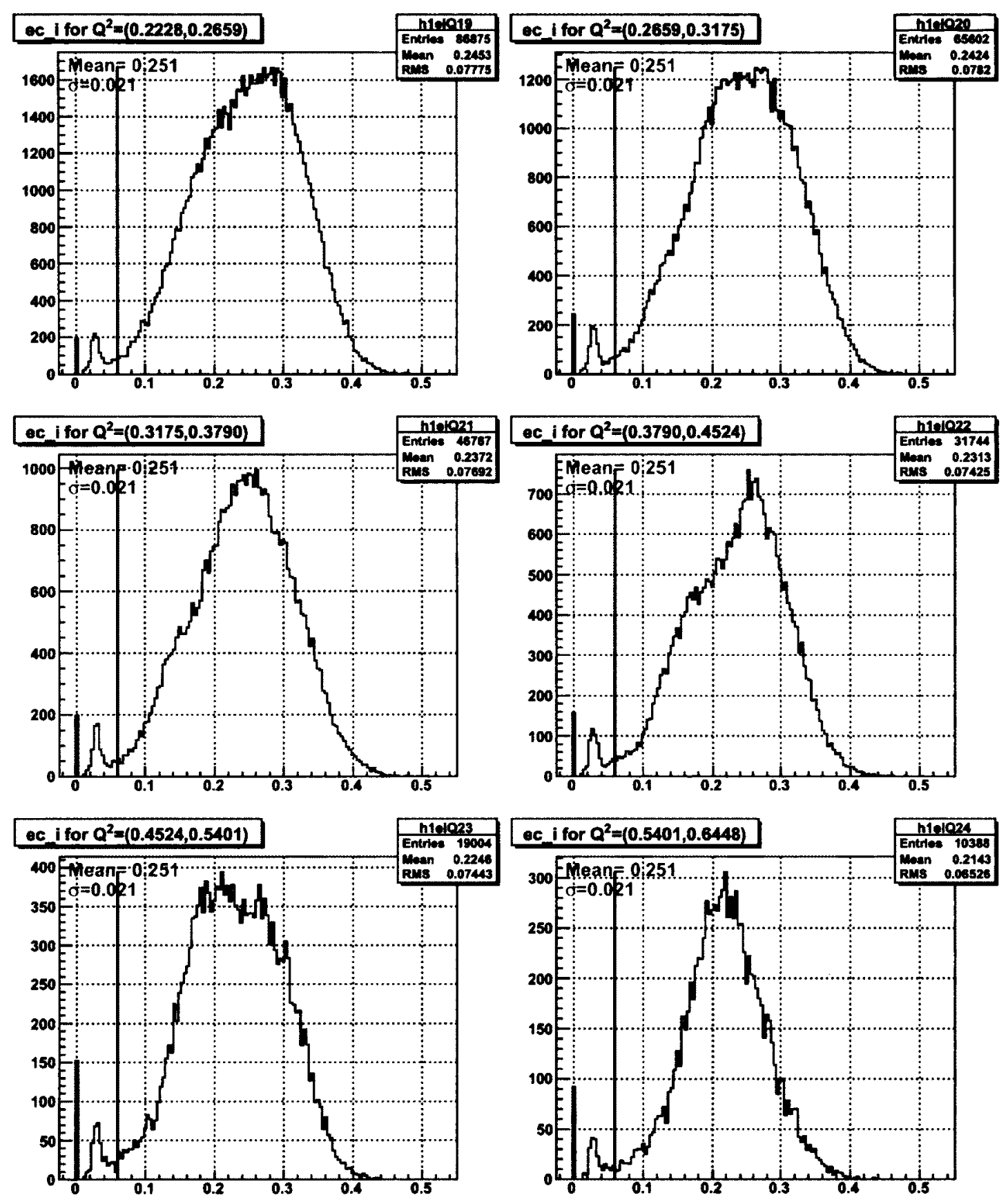

FIG. 46. The EC-inner cut on a sample of $2.0 \mathrm{GeV}$ experimental data in various $Q^{2}$ bins. 

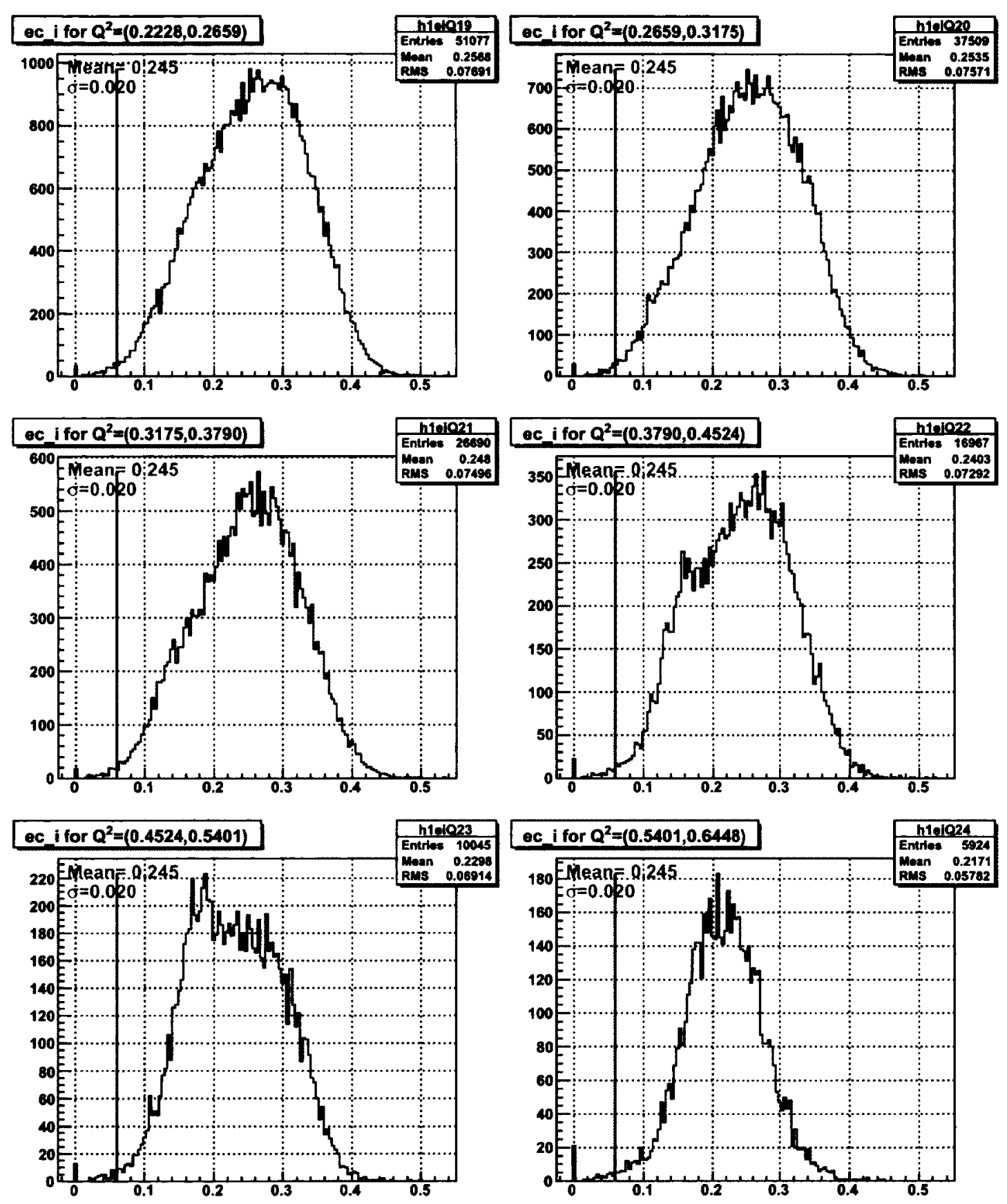

FIG. 47. The EC-inner cut on a sample of $2.0 \mathrm{GeV}$ simulation data in various $Q^{2}$ bins. 


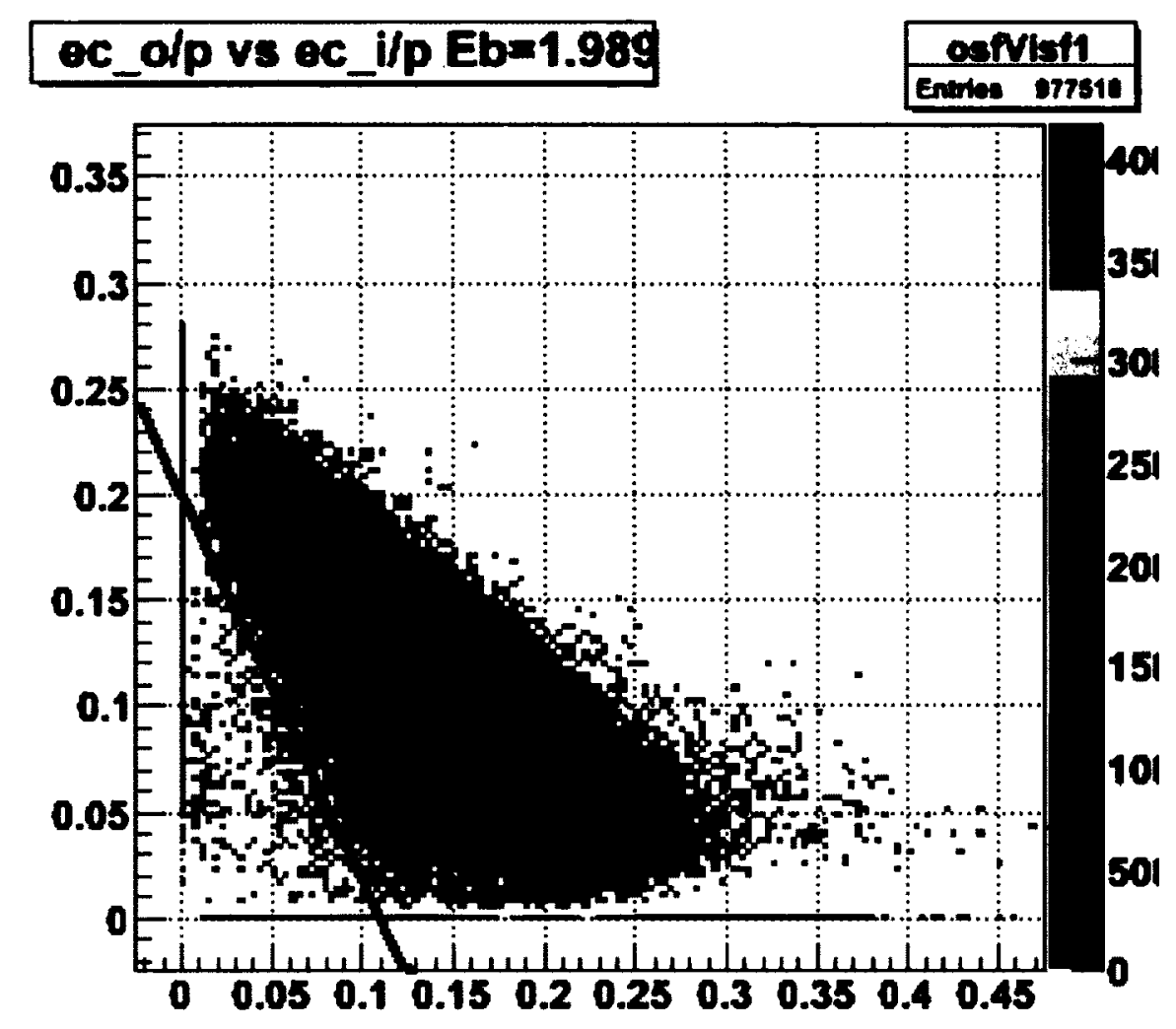

FIG. 48. Energy detected in EC outer as a function of EC inner, both normalized with the particle momentum, for the $2 \mathrm{GeV}$ data. The brown line shows the $\mathrm{EC}$ cut to reject pions (which fall below that line).

geometric and time matching cuts are applied.

The cuts in this category were worked out for this experiment by X. Zheng - one of the collaborators of the experiment. Her work, in turn, was mostly based on a similar analysis done for another CLAS experiment by M. Osipenko [86] in order to study the CC response and thereby develop a method to better discriminate electrons from pions.

The first requirement in the CC-matching is for the electron candidate track (as reconstructed by $\mathrm{DC}$ ) to have a corresponding signal in $\mathrm{CC}$. In addition, the track needs to meet several matching conditions to be acceptable as described in the next sections. 


\section{$\mathrm{CC} \theta$ Matching}

As said above, the $\mathrm{CC}$ segments are at different average polar angle positions (between 15 and 45 degrees), so in principle, one can expect a one-to-one correspondence between the polar angle of the track (as measured at the vertex) and the $\mathrm{CC}$-segment. However, the torus magnetic field bends the particles towards or away from the beamline, so it's more convenient to use the $\mathrm{CC}$ projected polar angle $\theta_{\text {proj }}$ rather than the vertex angle $\theta$, where $\theta_{\text {proj } j}$ is defined as the polar angle of the position vector defined by the point of intersection of the track with the CC plane (another projected angle $\phi_{p r o j}$ is the azimuthal angle of the same vector). These projected angles can be uniquely calculated for each track based on the DC signals of the track as well as the CC geometry information. To simplify the later analysis process, these projected angles for each track were calcuated during the final data reconstruction process and then saved in the output files just like the all other information for the events and particles. Finally, for the actual electrons a oneto-one correspondence between $\theta_{p r o j}$ and the segment number can be established, which discriminates against background noise and the accidental pions (or any other negative charge candidates). For each segment, the $\theta_{\text {proj }}$ distribution is fitted with a gaussian to determine its mean $(\mu)$ and width $(\sigma)$ and then saved for future use in cuts. These fit parameters are then used during the data analysis to define these CC- $\theta$-matching cuts. The events that have $\mu-3 \sigma<\theta_{\text {proj }}<\mu+3 \sigma$ pass this cut, and the others are rejected as not genuinely being electrons.

\section{CC $\phi$ Matching}

One can also have a one to one correspondence between the other $\mathrm{CC}$-projected angle $\phi_{p r o j}$ and the left or right PMT in the corresponding CC-segment, because when the track is on the right side of the $\mathrm{CC}$, the right PMT should fire and vice versa. However, there are some exceptional cases of events which fire both PMTs. That happens when $\phi_{p r o j}$ of the track is less than 4 degrees (when measured relative to the sector mid-plane), in which case the Cerenkov light hits both PMTs but with less efficiency (because the energy is shared between the two).

\section{Time Matching}

The difference $\Delta T$ between the track time recorded on a $C C$ segment and the corresponding time recorded on the TOF, corrected for the path length from the $\mathrm{CC}$ to the TOF, is used to 
define one of the time-matching cuts. Likewise, the time between $\mathrm{CC}$ and $\mathrm{EC}$ is also used to define another cut.

\subsubsection{CUT ON MINUMUM NUMBER OF PHOTOELECTRONS}

The "nphe" variable in the data ntuple which represents the ADC signal from the CC converted to "number of photoelectrons" and multiplied by 10 is also to discriminate electrons from pions and electronic noise. The number of photoelectrons produced in $\mathrm{CC}$ by an electron is typically between 5 and 25 or between 50 and 250 in the units of nphe, where the electronic background and negative pions produce signals equivalent to one photo-electron (or 10 in nphe units) and so a cut is determined somewhere between these two regions based on the shapes and sizes of the electron and pion peaks. In our case, we chose to have the cut at nphe $=25$ as depicted by the straight line in Fig. 49 .

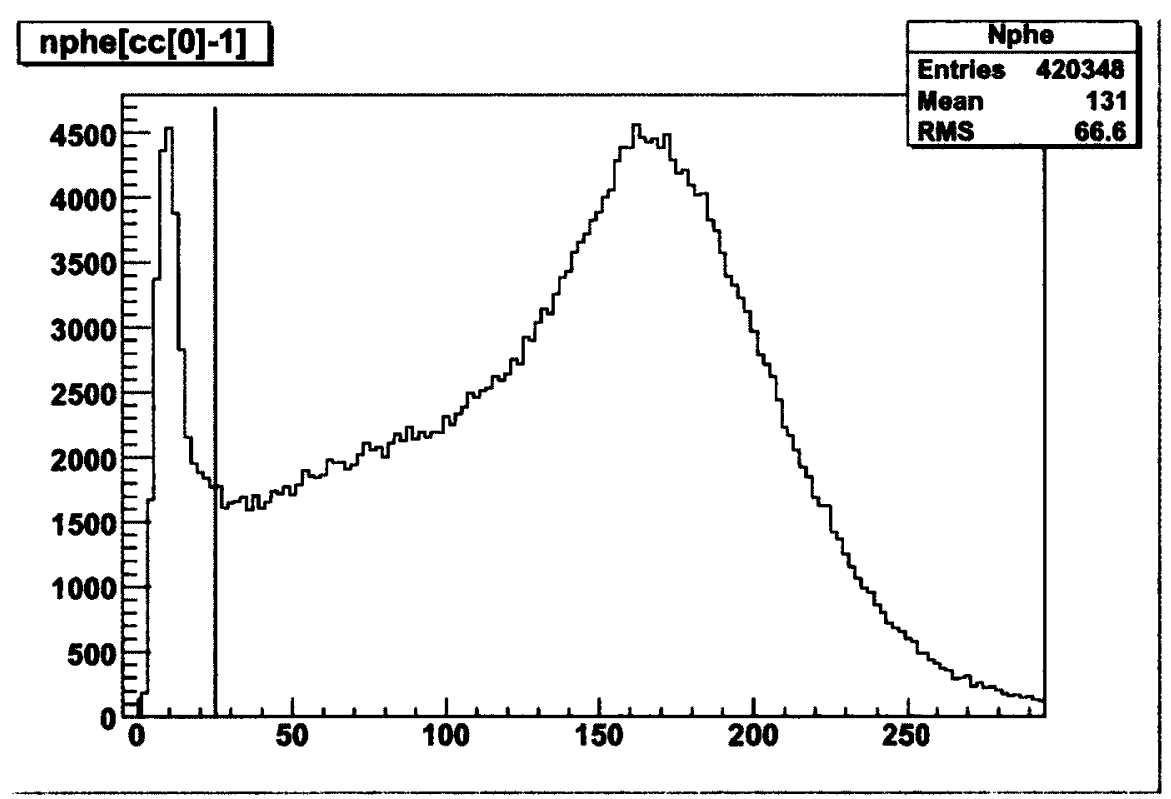

FIG. 49. The cut (the red straight line at 25) on the number of photo-electrons produced in CC times 10 (from $2.0 \mathrm{GeV}$ data). The signals below the red line are mostly pions and noise and above the line are mostly electrons. 


\subsubsection{MINIMUM/MAXIMUM MOMENTUM CUTS}

A study [87] of the inclusive cross section at various beam energies in CLAS developed a parametrization of the low momentum cut $p_{\min }$ as a function of the calorimeter low trigger threshold (in milli-Volts)

$$
p_{\min }(\mathrm{MeV})=214+2.47 \times E C_{\text {threshold }}(\mathrm{mV})
$$

The low threshold for EC-total energy for EG4 was $65 \mathrm{mV}$ [88], so, the minimum momentum cut was determined to be at: $p_{\min }=0.37 \approx 0.4 \mathrm{GeV}$. In addition, another minimum cut of $p_{\min }=0.2 * E_{\text {beam }}$ was added, so the actual minimum cut amounted to the larger of those two. Likewise, the momentum cannot be more than that of the beam energy (in natural units), therefore, the upper cut on the momentum is: $p_{\max }=E_{\text {beam }}$.

Fig. 50 shows the momentum distribution of the electron candidates for the $2 \mathrm{GeV}$ data and the minimum and maximum cuts.

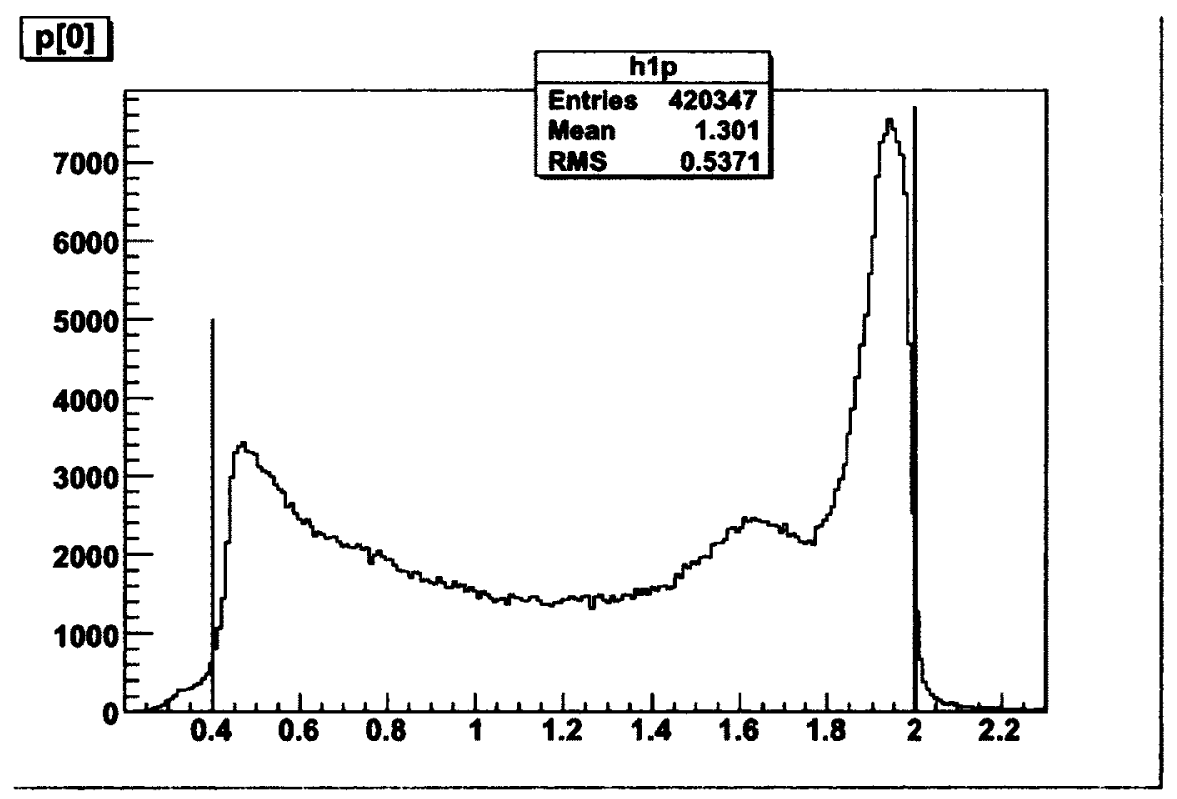

FIG. 50. The maximumum and minimum momentum cuts (on $2.0 \mathrm{GeV} \mathrm{ND}_{3}$ data). 


\subsubsection{VERTEX-Z CUTS}

In the EG4 experiment, the $\mathrm{ND}_{3}$ polarized target was of $1 \mathrm{~cm}$ long and was placed at $(x=0, y=0, z=-100.93 \mathrm{~cm})$ in the CLAS coordinate system. Since the beam electrons have to go through a few foils before reaching the target as well as the detector, we want to reject electron tracks with vertices outside the target volume. For this purpose, use a cut on the reconstructed vertex co-ordinate " $v_{z}$ ". However the vertex resolution demands resonably wide " $\mathrm{v}_{z}$ " cuts so as not to loose too many good events. That is why the distribution of " $v_{z}$ " was studied and based on the position and width of the distribution as well as our knowledge of the location of various foils and target materials, the cuts on " $v_{z}$ " were decided. It was seen (see Figs. 51 and 52) that the resolutions get worse and the distributions get wider as we go to lower $Q^{2}$ values, so again $Q^{2}$ dependent cuts were chosen for both data and simulation with the cuts tightening as $Q^{2}$ increases.

As in the case of EC variables, the reconstructed " $v_{z}$ " distribution in the simulation does not come out quite the same as in the experimental data. To have the same fraction of events in the corresponding $Q^{2}$ bins as in the experimental data, a separate set of cuts (determined based on the distributions of both types of data) had to be used for simulation. For this purpose, the Gaussian fit parameters $\mu$ and $\sigma$ (representing the mean and standard deviation) for all the $Q^{2}$ bins were tabulated separately for both data and simulation and separate sets of $\pm 3 \sigma$ cuts were determined for all bins. For example, if $\mu_{q}$ and $\sigma_{q}$ were the two Gaussian fit parameters for the $q^{t h} Q^{2}$ bin of either data or simulation, then the lower and upper cuts for " $\mathrm{v}_{z}$ " for that data set in the given $Q^{2}$ bin would be $\mu_{q}-3 \sigma_{q}$ and $\mu_{q}+3 \sigma_{q}$ respectively (as shown by the magenta vertical lines in Figs. 51 and 52 .

\subsubsection{FIDUCIAL CUTS}

Similar to the cuts discussed so far, we also had to match the region of good efficiency of the physical detector with the corresponding region from the simulation. For the experimental and simulation data to be comparable, they must have the same detector acceptance. Two event variables polar angle $\left(\theta_{v t x}\right)$ measured at the vertex and the azimuthal angle $\phi_{D C 1}$ measured at the drift chamber layer 1 are chosen to define the good efficiency regions of the detector. The reason for the choice of the variable $\theta_{v t x}$ should be obvious because it is directly related with the kinematic variables $Q^{2}$ and $W$ used in the analysis. However, due to the momentum dependent rotational effect of the magnetic field on the reconstructed 

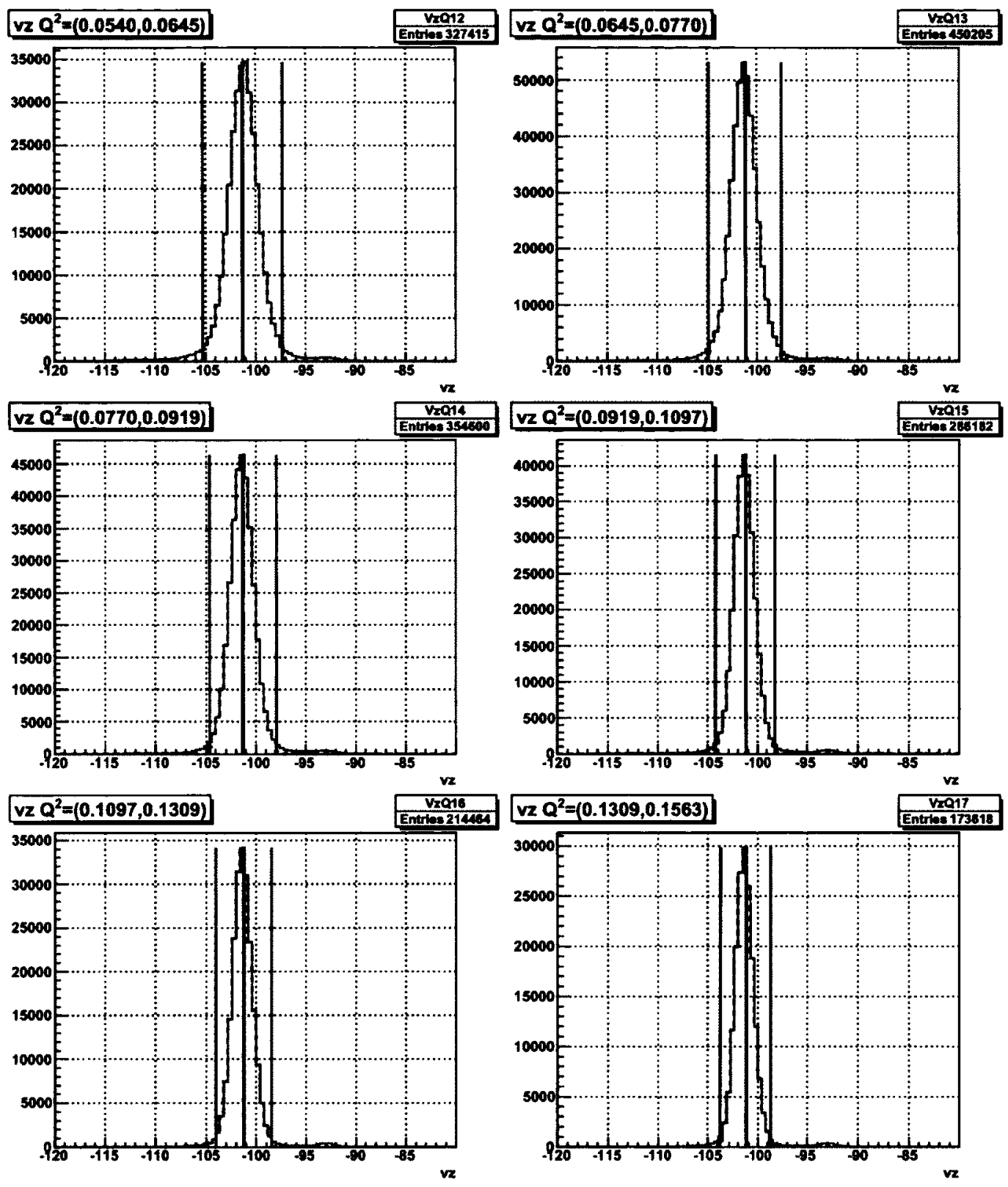

FIG. 51. $2.0 \mathrm{GeV}$ data showing the $Q^{2}$ dependent $\mathrm{v}_{z}$-cuts (the magenta lines on the left and right of the peaks) in some of the $Q^{2}$ bins. The continuous black line represents events before applying all the other event selection cuts (except on $\mathrm{v}_{z}$ ) and the thicker dotted red line are the events after the cuts. The blue lines are the centers of the distributions, from which the cuts are 3 times $\sigma$ away on each side, where $\sigma$ is the standard deviation for the distribution in the given $Q^{2}$ bin (both the central value and the $\sigma$ are determined during the cut development studies). 
azimuthal angle $\left(\phi_{v t x}\right)$ at the vertex, the angle $\phi_{D C l}$ is preferred over $\phi_{v t x}$ to define the fiducial region because that allows the easy selection (rejection) of the events which passed through and got detected by the more (less) reliable central (marginal) regions of the drift chambers. After a careful and extensive study of the event distributions on both data and simulation, we arrived at the fiducial cuts in terms of the two variables $\theta_{v t x}$ and $\phi_{D C 1}$ as shown by the magenta lines in Fig. 54.

In addition, the data and simulation were also directly compared with each other by taking the ratio of their distributions in a two dimensional space defined in terms of two variables $\theta_{v t x}$ and the torus current normalized inverse momentum (i.e. Itorus $/(2250 p)$. In one case, the ratio was taken between the regular experimental data and the "EC-only" experimental data (with CC-signal not required in the event trigger) (see Fig. 55) and in the other case, the ratio was of the experimental deuteron data (after background subtraction) to the simulated deuteron data (see Fig. 56). From these comparisons, some of the regions that showed big $\mathrm{CC}$-inefficiencies or big discrepancies between data and simulation were selected and removed from the fiducial region as indicated by various straight lines in the two plots. 


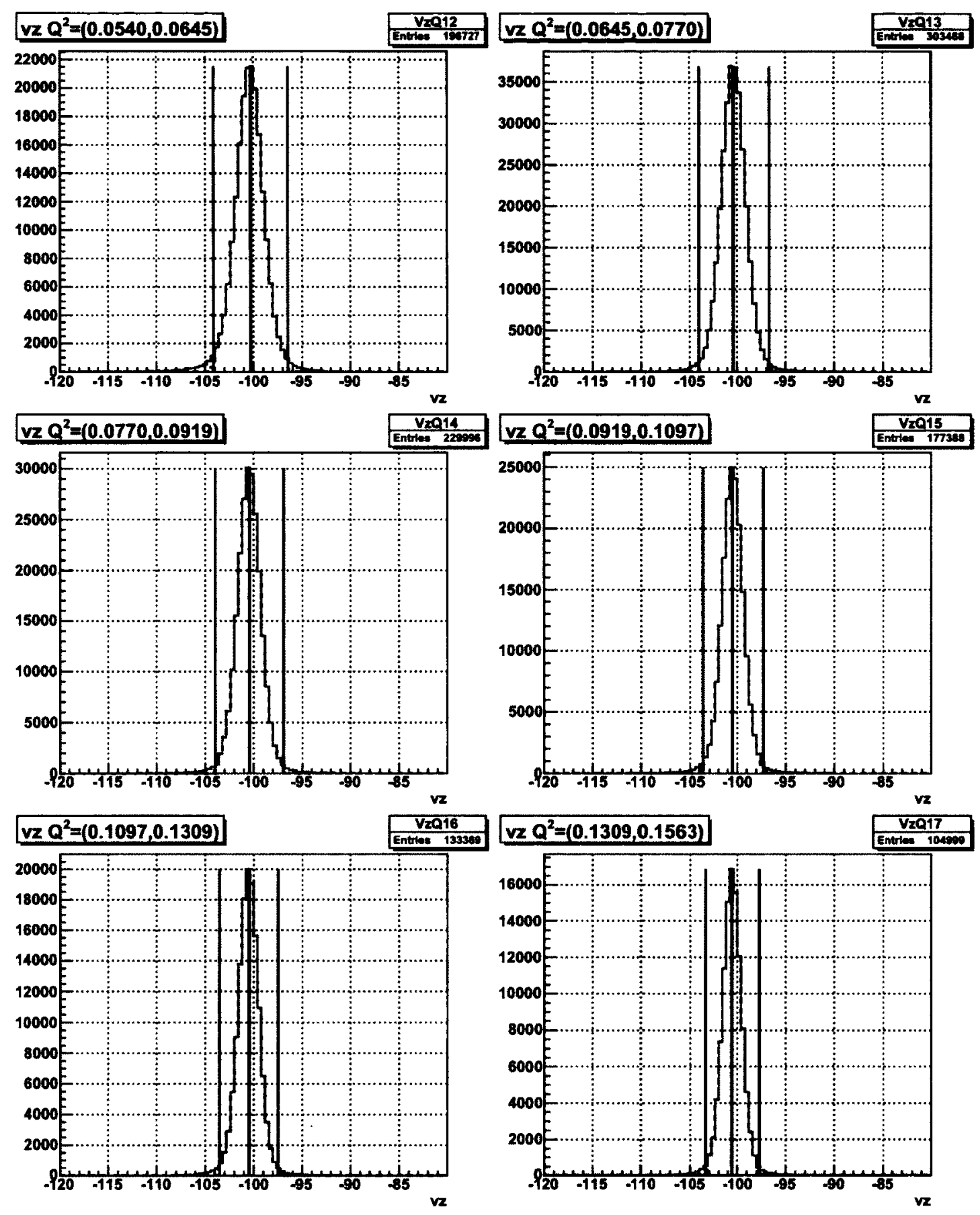

FIG. 52. $Q^{2}$ dependent $\mathbf{v}_{z}$-cuts on simulation data (similar to Fig. 51). 


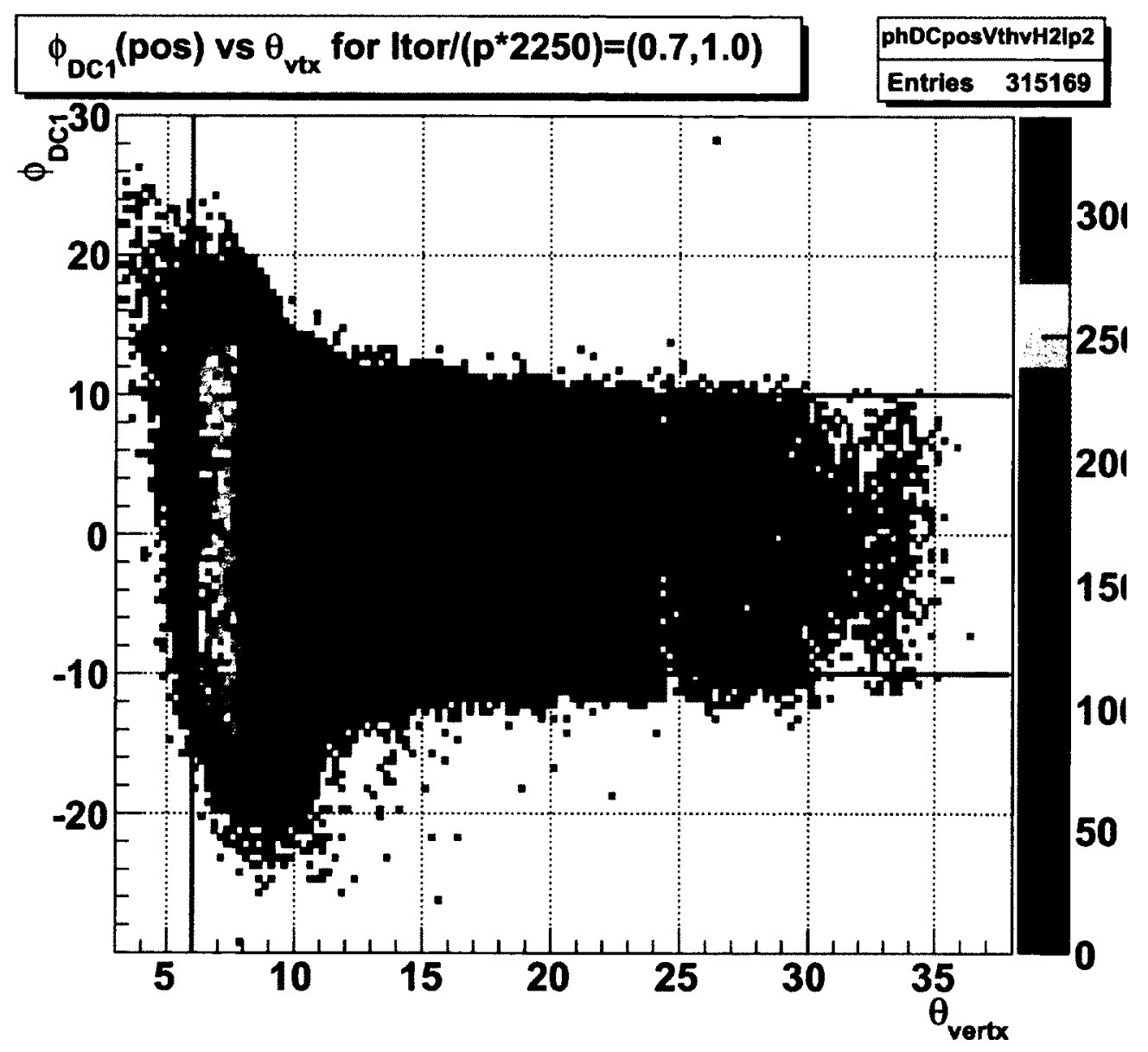

FIG. 53. Distribution of experimental data as a function of vertex angle $\theta$ and azimuthal angle $\phi_{D C 1}$ as measured by the track position at the first drift chamber layer (angles in degrees). The magenta lines indicate the fiducial cuts for accepting good electrons. 


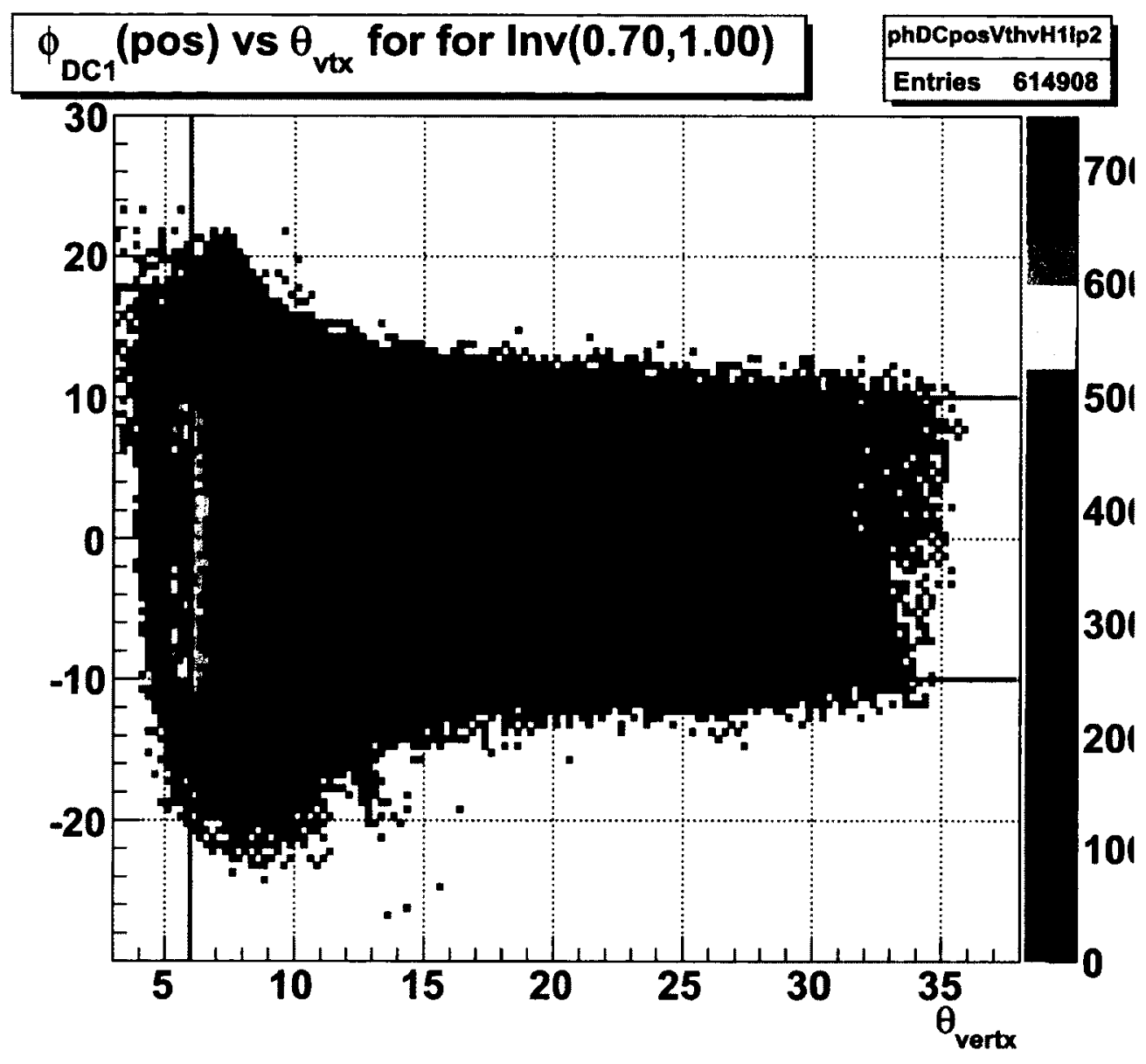

FIG. 54. Distribution of simulated data as a function of vertex angle $\theta$ and azimuthal angle $\phi_{D C 1}$ as measured by the track position at the first drift chamber layer (angles in degrees). The magenta lines indicate the fiducial cuts for accepting good electrons. 


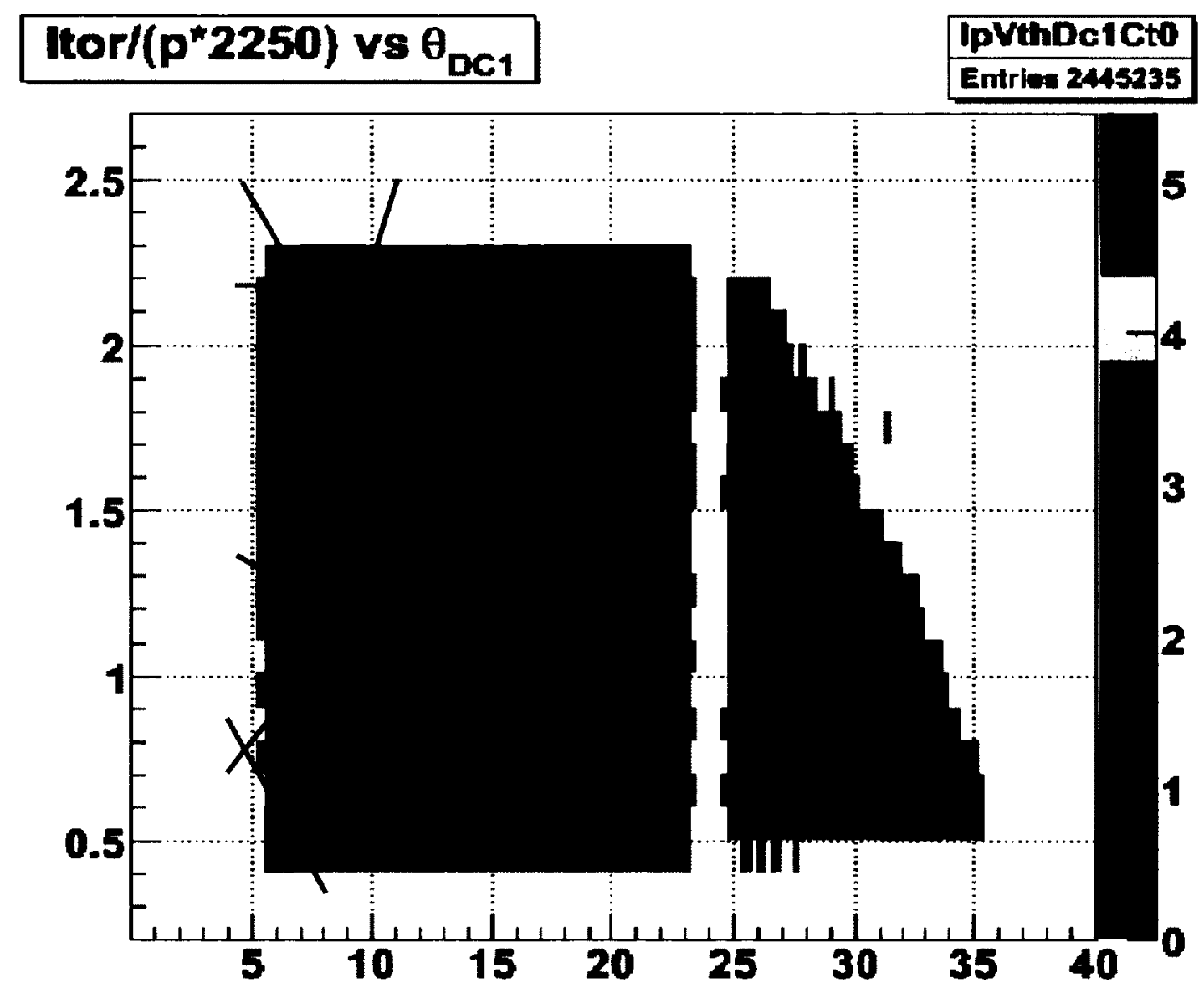

FIG. 55. Ratio of Regular to EC-only proton target data for $2.256 \mathrm{GeV}$ beam energy showing regions of varying CC-efficiencies in the $2 \mathrm{D}$ kinematic space defined by the scattering angle $\theta$ along $\mathrm{x}$-axis and the inverse momentum variable $\operatorname{inv} P=I$ tor $/(2250 p)$ along $\mathrm{y}$ axis. 


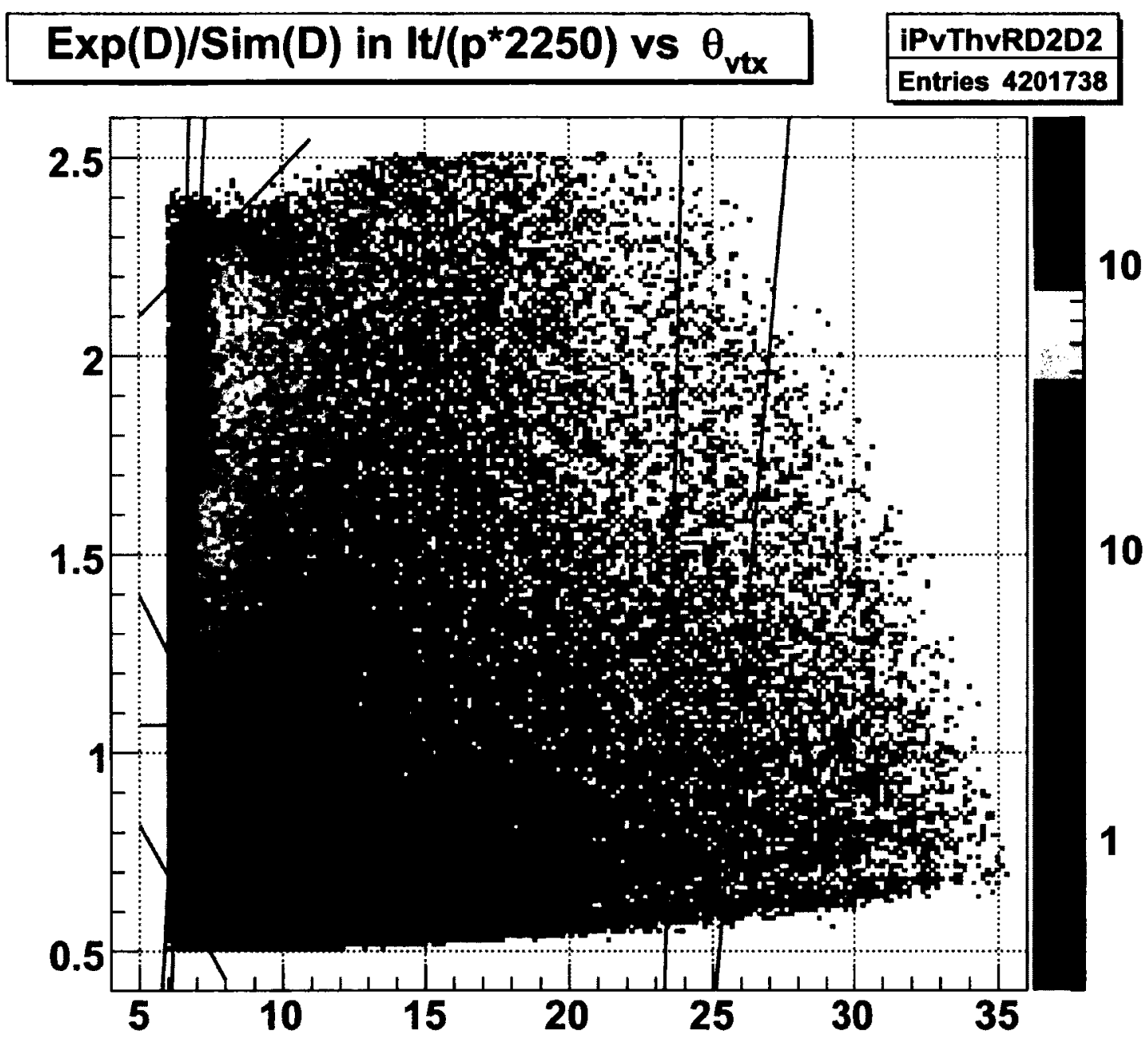

FIG. 56. Ratio of background subtracted $\mathrm{ND}_{3}$ (thus equivalent to deuteron) experimental data to simulated deuteron data (for $2.0 \mathrm{GeV}$ beam energy) as a function of $\mathrm{I}_{\text {torus }} / 2250 \mathrm{p}$ vs $\boldsymbol{\theta}$ at the vertex. The red and magenta lines indicate additional excluded regions. 


\subsection{CERENKOV COUNTER (CC) EFFICIENCY}

In the EG4 experiment, the Cherenkov Counter (CC) signal plays a major part in forming the event trigger for the data-acquisition system (DAQ). As stated earlier (see 3.4.4), for the purpose of achieving low $Q^{2}$ measurements with high detector efficiency ${ }^{3}$, a new dedicated $\mathrm{CC}$ was designed and placed in the sixth sector. Even though the new $\mathrm{CC}$ was designed to have a very high and uniform detection efficiency, some variation occurs over the covered kinematic range and therefore the knowledge of the detector efficiency as a function of the kinematics is required by our "method of absolute cross-section difference". Therefore, a study was done to determine the $\mathrm{CC}$ efficiency as follows.

\subsubsection{PROCEDURE}

It is assumed that the efficiency for some specific kinematic bin depends on the average number of photoelectrons produced by electrons in that bin which, in turn, is determined by the hit location on the Cerenkov PMT-projected plane as well as the angle with which the electron hits (or intersects) the plane. In the following, we describe how we determined the efficiency as a function of kinematic variables.

1. First, we define a torus-current normalized inverse-momentum variable $i p=\left(I_{t o r} / 2250\right) / p$ (see above), and divide the whole kinematic space into 12 bins in "ip" as follows: $(0.3,0.4,0.5,0.6,0.7,0.85,1.0,1.25,1.5,1.75,2.0,2.25,2.53)$. (For example, a 0.5 $\mathrm{GeV}$ electron during a $2 \mathrm{GeV}$ run, which used $2250 \mathrm{~A}$ for torus current, would have ip $=2.0 \mathrm{GeV}^{-1}$ )

2. Next, for each bin in "ip", a 2D map of the average number of photoelectrons is produced in a kinematic space defined by $\theta_{v t x}$ (scattering angle measured at the event vertex) and $\phi_{D C 1}$ (azimuthal angle as measured at DC1). For this step, some data from $\mathrm{NH}_{3}$ production runs ${ }^{4}$ are used with the standard electron selection cuts. One of these average-nphe maps is shown in the Fig. 57.

3. Next, using the "EC-only-trigger" data runs, good electron candidates are selected

\footnotetext{
${ }^{3}$ High detection efficiency is crucial for achieving smaller systematic uncertainties in the extracted physics quantities.

${ }^{4}$ This method relies on the use of two different sets of data. One is the regular $\mathrm{NH}_{3}$ target data and another is the "EC-only" data runs which were collected without using $\mathrm{CC}$ in the trigger. Since the latter type of data were collected with $\mathrm{NH}_{3}$ as target, to be consistent, $\mathrm{NH}_{3}$ production data was chosen rather than the $\mathrm{ND}_{3}$ ones to make the $N_{p h}$-maps.
} 


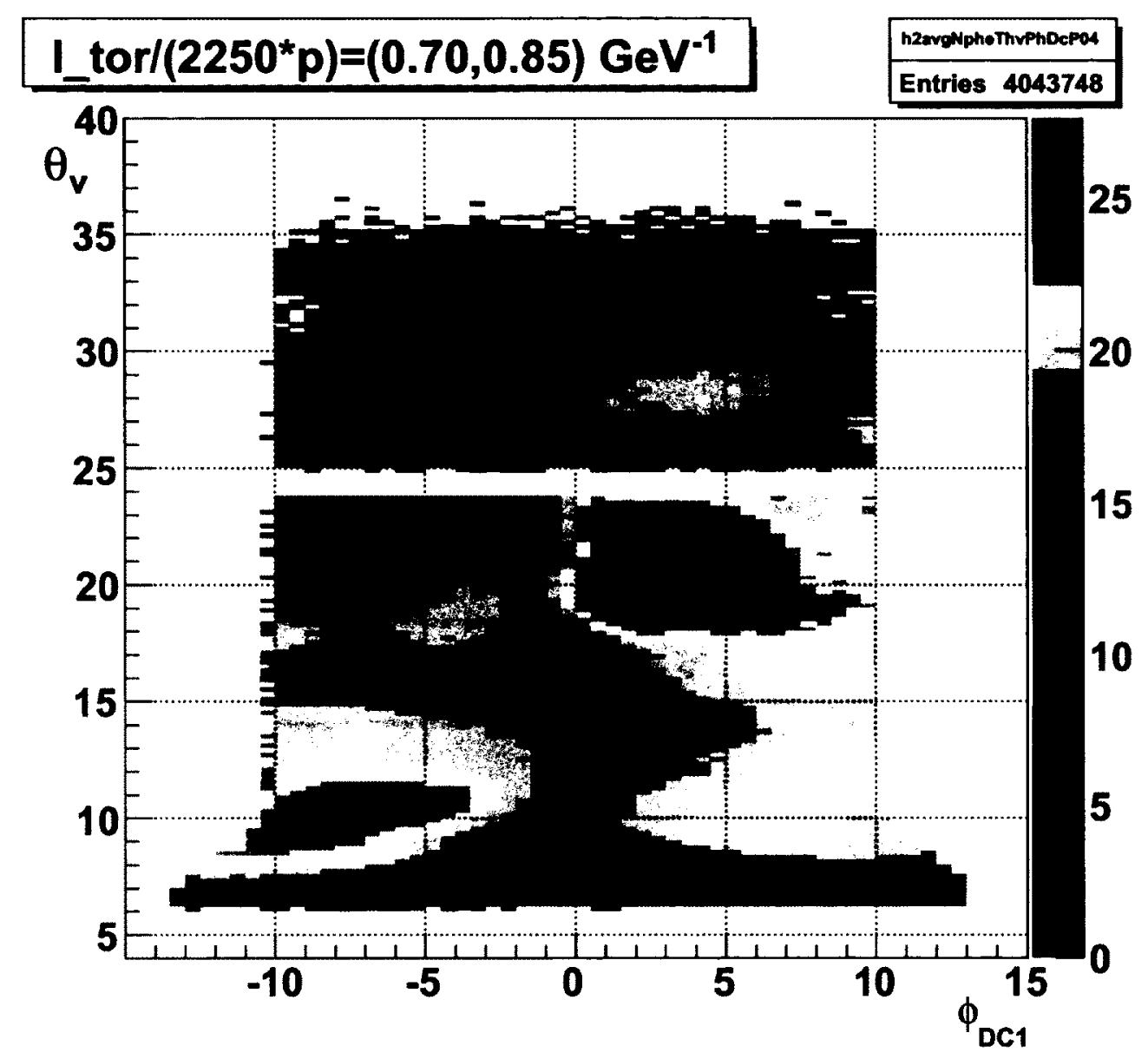

FIG. 57. Average photoelectron number (color-coded) produced in the 6th sector $\mathrm{CC}$ as a function of $\theta_{v t x}$ and $\phi_{D C 1}$ in the second bin of the variable $i p=\left(I_{t o r} / 2250\right) / p$ (from the $2.3 \mathrm{GeV} \mathrm{NH}_{3}$ data).

using the same cuts as before but without any CC-related cuts. For each of the selected electrons, the expected number of photoelectrons in the $\mathrm{CC}$ is determined in a look-up from the above average $N_{p h}$-maps based on its momentum and angles. This expected $N_{p h}$ is then histogrammed in two ways - one histogram for those electrons which either didn't trigger $\mathrm{CC}$ or didn't pass all of the $\mathrm{CC}$ related cuts and another histogram for all electrons. The ratio of these two histograms (shown in the top-right and top-left panels of Fig. 58 respectively) gives us the inefficiency of the CC-detector as a function of $N_{p h}$ (as shown by the bottom two panels of the same figure). (Errors in the inefficiencies have not been drawn (for the purpose of 
cleaning) in the figures but they were calculated using the fact that the error in a ratio $\mathrm{N} 2 / \mathrm{N} 1$ is $\sqrt{N 2(1-N 2 / N 1)} / N 1)$.

4. The ideally expected CC intrinsic inefficiency is given by the Poisson distribution, since we require more than 2 photoelectrons, the theoretical prediction for the inefficiency is actually $\left(1+N_{p h}+1 / 2 N_{p h}^{2}\right)^{*} \exp \left(-N_{p h}\right)$. However, we found empirically that if we calculate $N_{p h}$ only with electrons that exceed the threshold of 2.5 , then we find that the functional form is pretty close to the form $y=p_{0}+p_{1} \cdot \exp \left(-p_{2} x\right)$, where $\mathrm{x}$ represents $\left\langle N_{p h}\right\rangle$, and $\mathrm{y}$ represents the inefficiency. This form was used to fit with the above measured inefficiency and the result of the fit is shown in Fig. 58. We find that the inefficiency agrees very well with the expectation at low nphe, but remains at a very small constant value of around 0.01 (we call it the "constant background") at higher nphe.

5. Finally we use the inefficiency fit just developed to evaluate the corresponding efficiencies and transform the 2D map of $N_{p h}$ into the corresponding efficiency maps (see Fig. 59 for such a map in one momentum bin.). These maps are later used to apply the efficiency correction on an event by event basis in the simulation.

From this study, we see that the $\mathrm{CC}$ is very efficient in most of the kinematic region (see Fig. 59). Once, the CC-(in)efficiency was estimated, we use the calculated CC efficiency to multiply our simulation (i.e., for each simulated event, we look up the CC efficiency and weigh the event with it. 

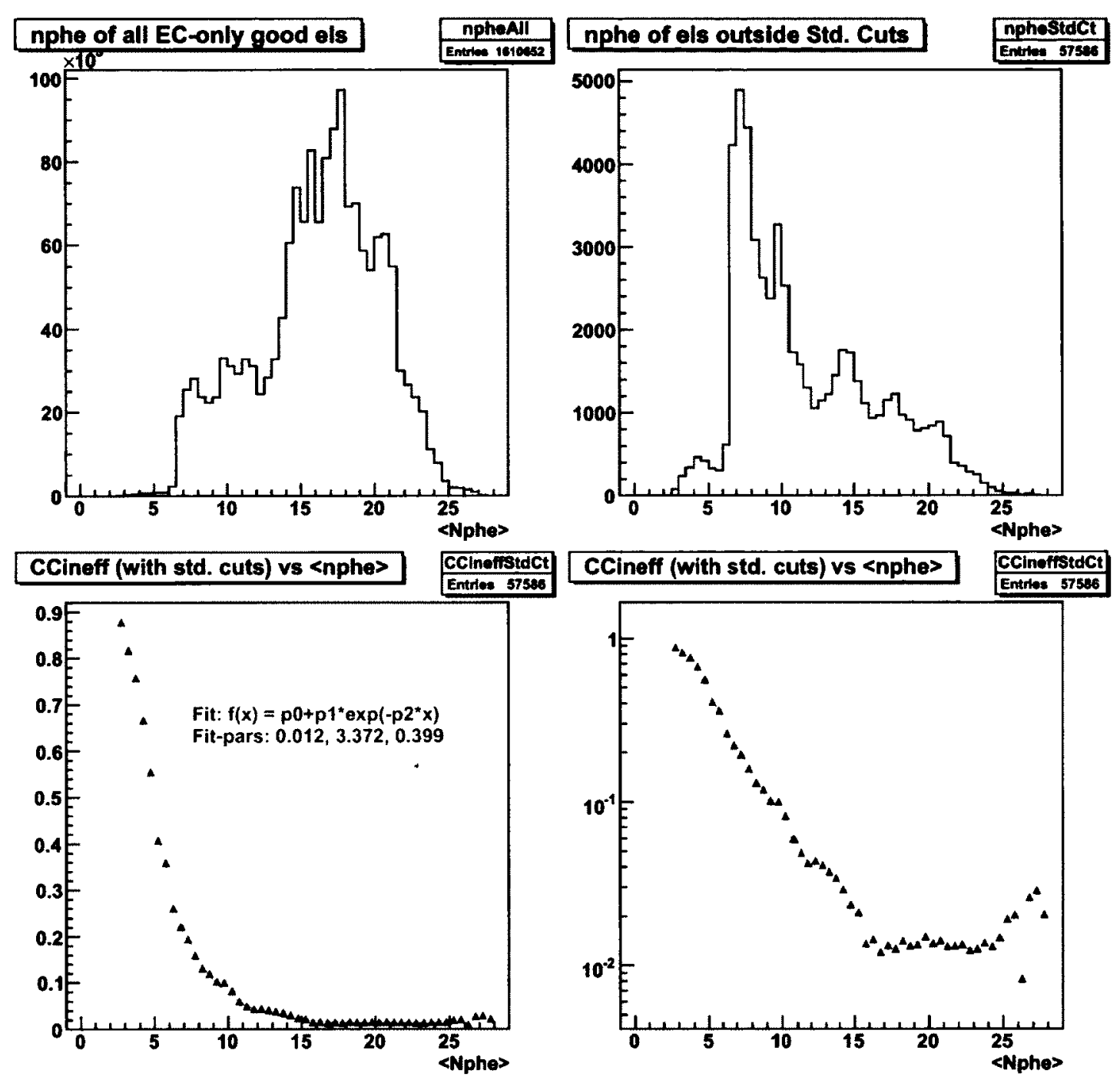

FIG. 58. EC detected good electrons (for all momenta) as a function of $\left\langle N_{p h}>\right.$ (top left). Similar distribution (top right) for those good electrons that were detected by the EC but were rejected by the standard set of event selection cuts which includes $\mathrm{CC}$-dependent cuts. By dividing the latter with the former, one gets the calculated $\mathrm{CC}$ inefficiency. The bottom two plots show the inefficiency distribution and a fit (red continuous line) in both linear (in third panel) and logarithmic (fourth panel) scales. Looking at the first plot, it can be seen that most electrons are above $N_{p h}=15$ where the inefficiency is at most $1-2 \%$. 


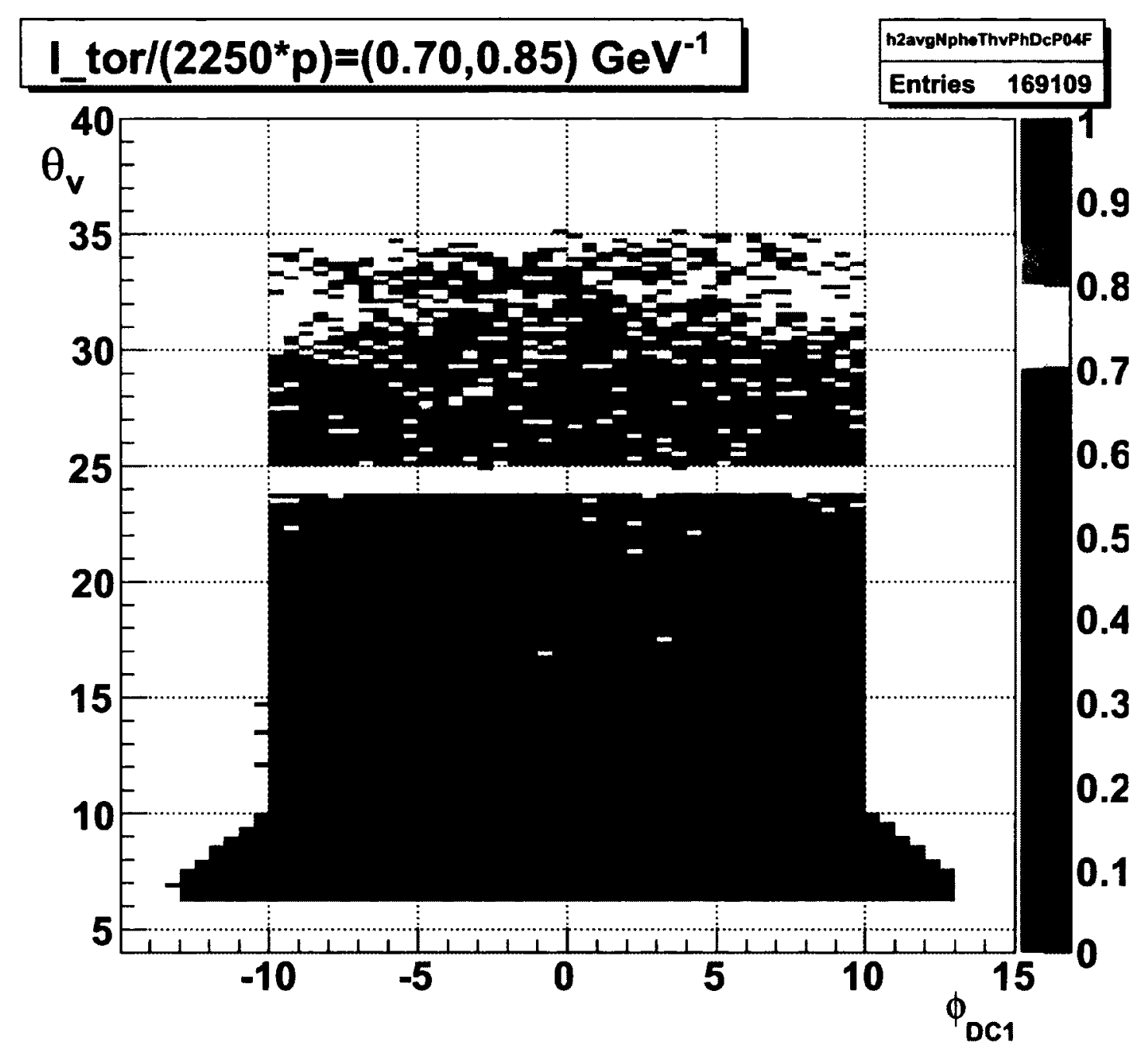

FIG. 59. CC-efficiency in a momentum bin . 


\subsection{PION CONTAMINATION CORRECTIONS}

One of the two major sources of backgrounds in the measured EG4 electron rates comes from misidentified negatively charged pions $\left(\pi^{-}\right)$that produce similar set of signals as electrons in various detector components and thus pass the electron ID cuts. In the EG4 experiment, signals from the electromagnetic calorimeter (EC) and Cherenkov counter (CC) are used to discriminate electrons from pions, but even with stringent conditions on these signals, some of the pions get misidentified as electrons. To avoid limiting statistics too much in order to minimize the final statistical error in a given kinematic bin, a tradeoff in purity versus efficiency (statistics) is made by quantifying the amount of this kind of contamination.

\subsubsection{METHOD}

First, the whole kinematic space covered by EG4 is divided into 90 two-dimensional bins -9 in $p$ and 10 in $\theta^{5}$.

For each kinematic bin, a histogram of the number of photo-electrons (variable ' $N_{p h e}$ ' in the data ntuple) produced by the electron candidates (selected using the standard particle selection conditions (cuts) except that no cut on ' $N_{\text {phe }}$ ' is included is made (see Fig. 4.8.1). Likewise, using a very stringent set of cuts, a similar histogram is made for the cleanest possible sample of pion candidates in the same kinematic bin.

- Estimating the contamination in each bin: A 7 th order polynomial is fit to the $N_{\text {phe }}$ histogram for electrons in the $N_{p h e}$ range extending from $N_{p h e}=1.8$ to $N_{p h e}=10$. The fit is then extrapolated down to $N_{\text {phe }}=0$ (see Fig. 4.8.1). Subtracting the extrapolated fit from the impure electron distribution results in the extraction of the contaminating pion peak ${ }^{6}$. Rescaling the pure pion sample to the extracted peak gives us the distribution of the actual pion contamination over the complete range of $N_{p h e}$. Finally, the

\footnotetext{
${ }^{5}$ For $2 \mathrm{GeV}$ or higher beam energy data sets, the p-bin boundaries are chosen as $(0.30,0.60,0.90,1.20$, $1.50,1.80,2.20,2.60,3.00)$ and $(0.30,0.45,0.60,0.75,0.9,1.1,1.4)$ for others. And, for $\theta$, the boundaries are $(5.0,6.0,7.0,8.0,9.0,10.0,12.0,15.0,19.0,25,49)$. The choice of the binning was rather arbitrary. Nevertheless higher statistics region was divided into relatively finer bins (event population peaks around $\theta=$ 10 degrees).

${ }^{6}$ Beyond $N_{p h e}=1.8$, the electron sample is nearly pure except for a tiny fraction due to the pion tail, so any function that fits that section of the $N_{p h e}$-distribution is supposed to represent the pure electron distribution. In order to simplify the situation, we chose to fit only from 1.8 to 7.0 rather than covering the full range beyond 7.0.
} 
counts corresponding to this rescaled pure sample in the region above the standard cut $N_{p h e}>2.5$ is calculated. Then the ratio of this count to the impure electron count in the same standard $N_{\text {phe }}$ range gives the measured contamination for the bin.

- The contaminations thus evaluated for different momentum bins belonging to a particular $\theta$-bin are then plotted against the corresponding momenta. Then, this is fit to an exponential function.

- The parameters par1 and par2 of the exponential fit performed in different theta bins are next graphed together to see the presumed linear dependence.

- Finally, a global fit is performed on all the contaminations in different $\theta$ - and p-bins (not on the fit parameters). The fit parameters from the earlier two fits only give us a hint to the type of the dependence, thus allowing us decide the form of the fit function.
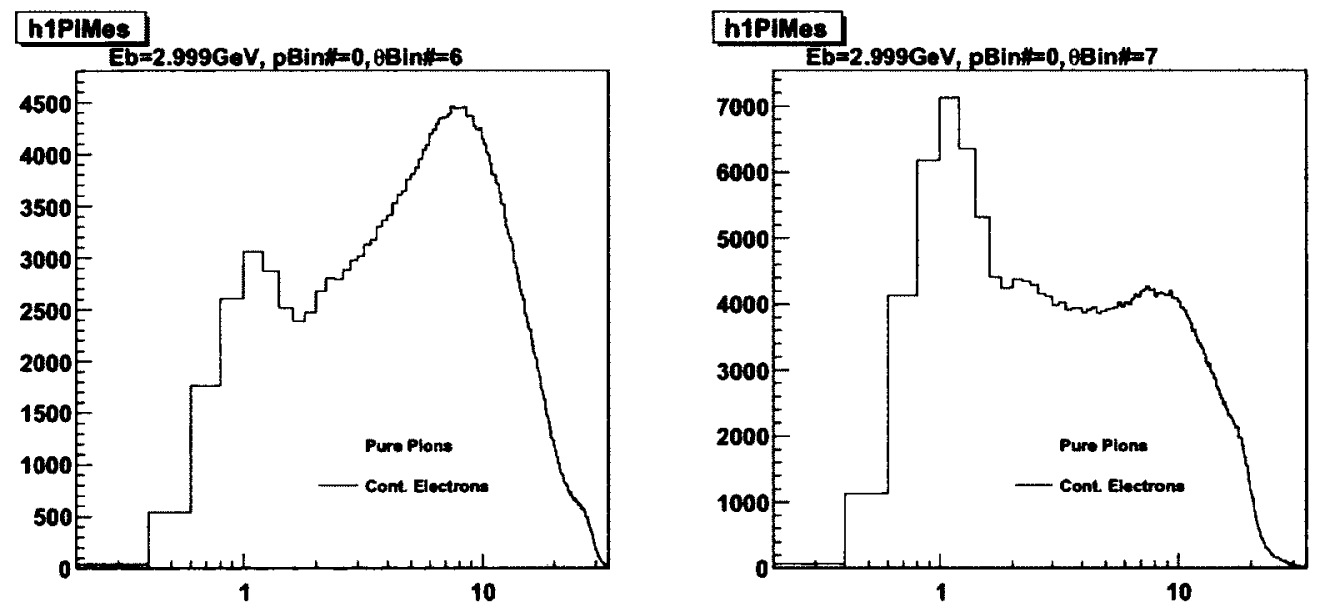

(a) For the first in momentum and seventh in $\theta$ bin. (b) For the first in momentum and eighth in $\theta$ bin.

FIG. 60. Number of photo-electrons produced in $\mathrm{CC}$ by clean pion and contaminated electron samples (3.0 GeV data)

From the study, it is found that the typically pion contamination is less than $1 \%$. 


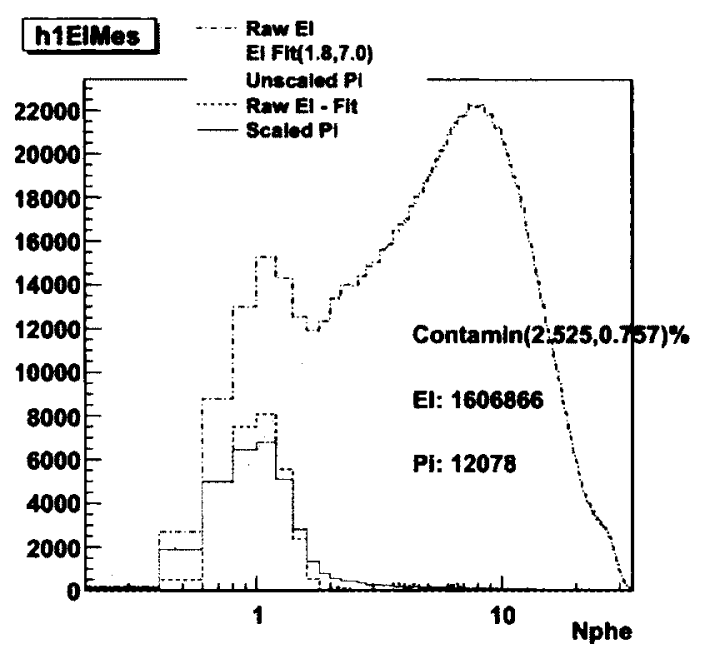

(a) For the first bin in momentum and seventh bin in $\boldsymbol{\theta}$.

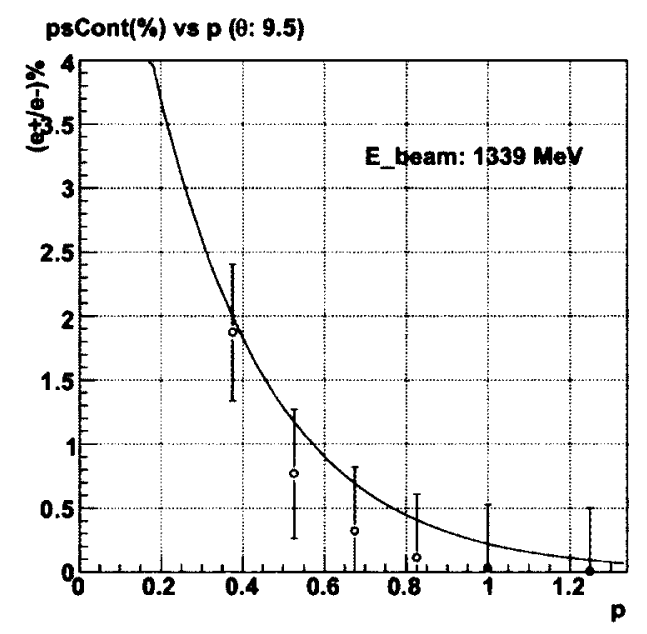

(c) Fits in the $\theta(9.0,10.0)$ bin for $1.339 \mathrm{GeV}$ data.

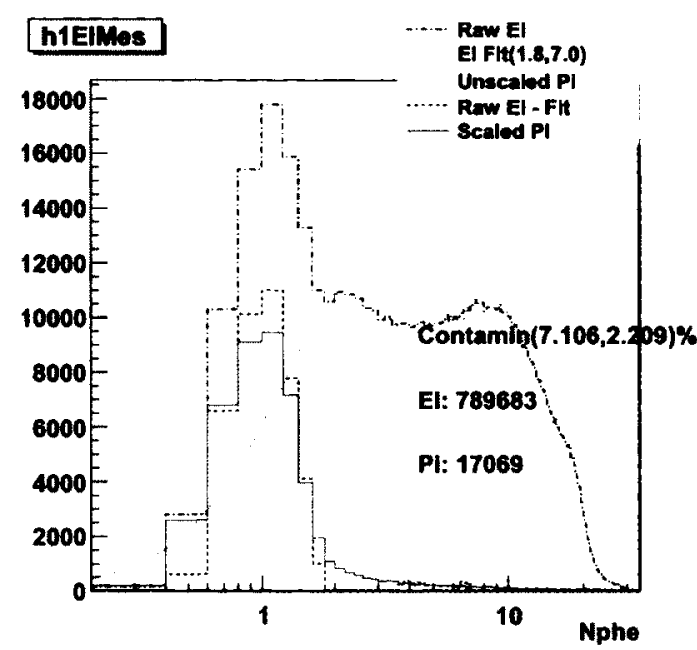

(b) For the first bin in momentum and eighth bin in $\theta$.

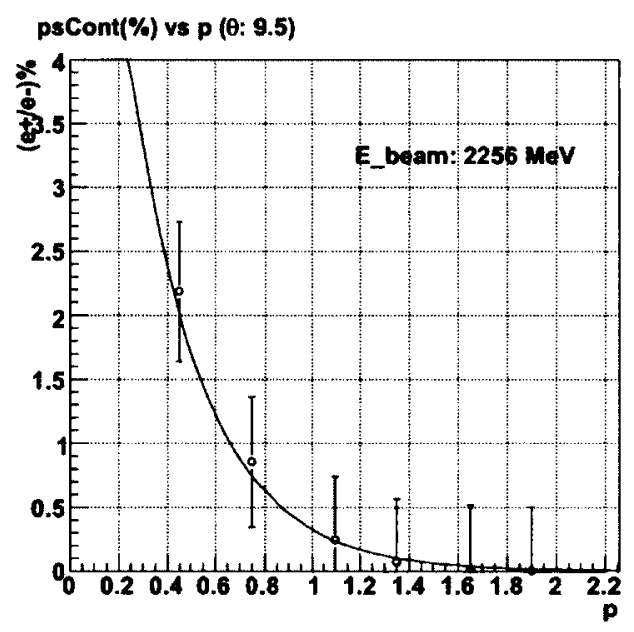

(d) Fits in the $\theta(9.0,10.0)$ bin for $2.0 \mathrm{GeV}$ data.

FIG. 61. The top row plots show the calculation of pion contamination of electrons for the given kinematic bins of $3.0 \mathrm{GeV}$ data. The dotted black line indicated by the label "Raw El" in the legends of each of the two plots are the contaminated electrons. Likewise, the line labeled "El Fit" is a polynomial fit to the electron distribution (in this case fitted from Nphe $=1.8$ to 7.0, but extrapolated down to $\mathrm{Nphe}=0$ ). The line labeled "Unscaled $\mathrm{Pi}$ ' is the pure pion distribution obtained with stringent set of cuts. "Raw El - Fit" is the difference between the contaminated electron sample and the polynomial fit and finally "Scaled Pi" is the pure pion-sample but after its scaled to match with the "Raw El - Fit" at the pion peak position (around $1 \mathrm{Nphe)}$. The bottom row plots show the fits of the contaminations as a functions of momentum $(p)$ in a given $\theta$ bin. 


\section{$4.9 e^{+} e^{-}$-PAIR SYMMETRIC CONTAMINATION CORRECTIONS}

The next major source of background is the secondary electrons from various $e^{+} e^{-}$ pair production processes. When an electron originating from such a pair passes through the detector, the detector has no way to distinguish it from the electrons that actually scattered off the target. Therefore, the detector simply accepts it as a true scattered electron candidate, thus producing a contamination that has to be estimated and corrected for. The first such source is the wide-angle $e^{+} e^{-}$pair production from bremsstrahlung photons generated in the target. The other major source is hadron decay such as the Dalitz decay $\left(\pi^{0} \rightarrow e^{+} e^{-} \gamma\right), \pi^{0} \rightarrow \gamma \gamma$ and then conversion of these photons into $e^{+} e^{-}$pairs. Likewise, the pseudoscalar particle $\eta$, and the vector mesons $\rho, \omega, \phi$ also decay to $e^{+} e^{-}$, but they are not major contributors because of their very small decay probabilities as well as the small population compared to the $\pi^{0}$ and photons. Of all these sources, the biggest contributor to the secondary electrons is the $\pi^{0} \rightarrow \gamma \gamma$ with $\gamma$ conversion to $e^{+} e^{-}$[89].

The amount of contamination from this type of process can be estimated by monitoring the amount of positrons that were recorded under the same experimental and kinematic conditions. Because of the symmetry in the amount of electrons and positrons produced from these sources, the positron to electron ratio gives us the amount of the pair-symmetric contamination. However, due to the presence of the strong magnetic field inside the detector and the fact that the positrons have opposite charges, their detector acceptance would be different in a given setting. By reversing the magnetic field while keeping everything else the same, it is possible to estimate the contamination. For some of the beam energies used for the $\mathrm{NH}_{3}$ data $\mathrm{f}$ the EG4 experiment, some data were collected with identical experimental setting but with the torus field reversed. The data from those runs were used to estimate the amount of positrons in somewhat the same fashion as pion contamination. For example, Fig. 62 shows one estimate (both data points and the fit) of the contamination in EG4 compared with those determined for the EGlb experiment [35].

For this analysis, both the pion and $e^{+} e^{-}$pair symmetric contaminations are small enough to be ignored. This leads to only a slight increase in the systematic error in the final physics results. 


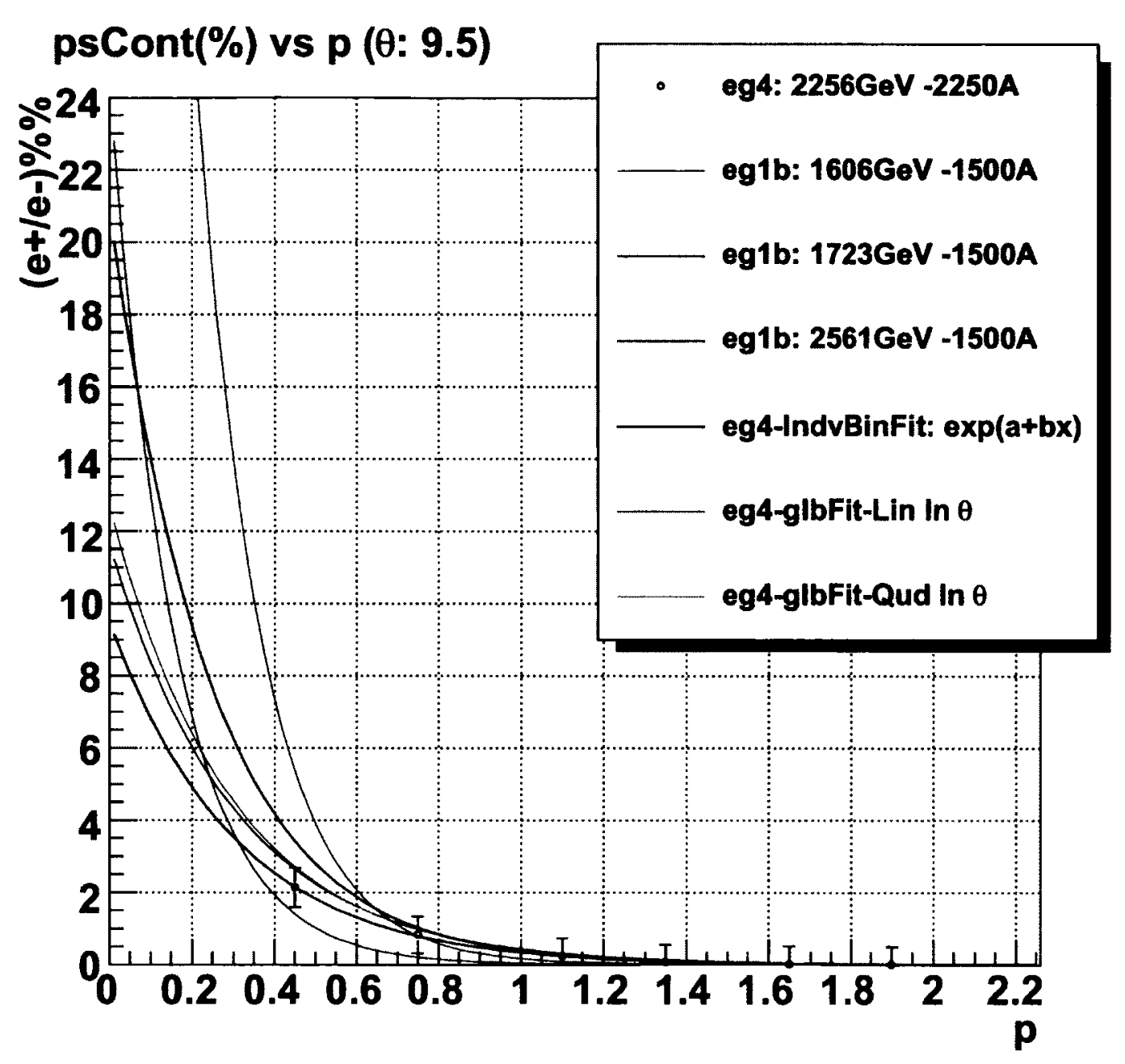

FIG. 62. Pair-symmetric contamination Fits (\%) as a function of electron momentum.

\subsection{STUDY OF $\mathrm{NH}_{3}$ CONTAMINATION OF EG4 ND NARGET $_{3}$ TAO}

In equation (102), it is assumed that the ammonia target is $100 \%$ pure i.e. composed of only ${ }^{15} \mathrm{ND}_{3}$ molecules and that the contribution from the slightly polarized nitrogen is negligible. But, in practice, the standard $\mathrm{ND}_{3}$ sample is not a $100 \%$ pure material. Rather, it contains one or two percent of ${ }^{14} \mathrm{ND}_{3},{ }^{15} \mathrm{NH}_{3}$ [90], and some traces of other isotopic species of ammonia. It was reported by the EG1-DVCS experiment at Jlab [91][92] that a higher than usual amount of $\mathrm{NH}_{3}$ (about $10 \%$ ) was observed in the $\mathrm{ND}_{3}$ target, indicating that an inadvertent mix-up of $\mathrm{NH}_{3}$ and $\mathrm{ND}_{3}$ materials could have happened during the 
experimental run. Wondering if the EG4 experiment had a similar incident, we decided to investigate and estimate the amount of $\mathrm{NH}_{3}$ contamination of our $\mathrm{ND}_{3}$ target by looking at the data from the $\mathrm{ND}_{3}$ run period of the experiment as described below.

\subsubsection{PROCEDURE}

The method involves using ep elastic (or quasi-elastic in the case of non-proton target) events and comparing the width in some quantity that reflects the correlation between the scattered electron (e) and the recoiling proton (p) due to the kinematic constraints of such events. The most suitable correlation is the one between the polar angles of the electron and the proton. That is because of the better angular resolution in CLAS than that for momentum, and also due to the fact that polar angle $(\theta)$ resolution is much better than that of the azimuthal angle $(\phi)$ because of the rotational effect (on $\phi$ ) of the polarized target field as well as the drift chamber resolutions [91].

The $\boldsymbol{\theta}$-correlation can be studied mainly in two ways. The first way is to reconstruct and histogram the beam energy using the measured polar angles and the known target mass and then compare the histogram from the $\mathrm{ND}_{3}$ target run with that from a pure $\mathrm{NH}_{3}$ target run. The other equivalent way is to predict the proton polar angles (using the measured electron angles, known target mass and the beam energy) and then histogram the deviation of the measured proton angles from the expected values. We chose to use a slightly modified version of the latter approach in which we histogram the following quantity ${ }^{7}$ :

$$
\Delta=p_{p} \cdot\left(\sin \theta_{q}-\sin \theta_{p}\right)
$$

where $p_{p}$ is the measured proton momentum, $\theta_{p}$ is the measured polar angle of the proton, and $\theta_{q}$ is the expected polar angle of the recoiling proton (which is also the angle of the exchanged virtual photon $(q))$ given by:

$$
\theta_{q}=\tan ^{-1}\left(\frac{M_{p}}{\tan (\theta / 2) \cdot\left(E_{\text {beam }}+M_{p}\right)}\right)
$$

The method exploits the fact that the width of the quantity $\Delta$ from data with deuteron target decreases because the Fermi motion of the protons in the deuteron nuclei gives a spread of the order of $50 \mathrm{MeV}$ in transverse momentum, and for longitudinal particle momenta of order of a few GeV, we obtain a polar angle spread about $20 \mathrm{mr}$, which is much larger than the intrinsic CLAS resolution of about $2 \mathrm{mr}$.

\footnotetext{
${ }^{7}$ We chose this quantity $\Delta$ rather than the simple angle difference $\left(\theta_{q}-\theta_{p}\right)$ because the former is more directly interpretable in terms of transverse missing momentum for the case of quasi-elastic scattering.
} 


\subsubsection{EVENT SELECTION}

First, for each data set (corresponding either to $\mathrm{NH}_{3}, \mathrm{ND}_{3}$ or ${ }^{12} \mathrm{C}$ runs), using standard electron and proton identification cuts events each with a well reconstructed scattered electron and a similarly well reconstructed candidate for proton are selected. We accept only events each of which have one electron, one proton and at most one neutral particle candidate (expected to be a neutron coming off from the deuteron target break-up). If the event is one of the above two types, following additional cuts are applied to make sure it is elastic or quasi-elastic event:

- $E_{X}<0.15 \mathrm{GeV} \quad$ with $E_{X}=M_{p}+E_{e}-E_{e^{\prime}}-E_{p}=M_{p}+v-E_{p}$

- $P_{X}<0.5 \mathrm{GeV} / \mathrm{c} \quad$ with $\vec{P}_{X}=\overrightarrow{0}_{p}+\vec{P}_{e}-\vec{P}_{e^{\prime}}-\vec{P}_{p^{\prime}}=\vec{P}_{e}-\vec{P}_{e^{\prime}}-\vec{P}_{p^{\prime}}$

- $0.88 \mathrm{GeV}<M_{X}<1.04 \mathrm{GeV}$

- $\theta_{q}<49.0^{\circ}$

- ||$\phi_{e}-\phi_{p}\left|-180.0^{\circ}\right|<2.0^{\circ}$

where $X$ indicates the missing entity in the $d\left(e, e^{\prime} p\right) X$ channel, which is expected to be neutron in the case of the quasi-elastic channel, thus $E_{X}$ is the missing energy and so on.

If it passes these cuts, the quantity $\Delta$ is calculated for the event and then histogrammed as shown by the red curves in the top-left (from ${ }^{12} \mathrm{C}$ runs), top-right (from $\mathrm{NH}_{3}$ runs), and bottom-right (from $\mathrm{ND}_{3}$ runs) panels of Fig. 63.

After getting the histograms for the quantity $\Delta$ for the ep-elastic or quasi-elastic events from the $\mathrm{NH}_{3}, \mathrm{ND}_{3}$ and ${ }^{12} \mathrm{C}$ target data sets, we first remove the contribution from the nonhydrogen component of $\mathrm{NH}_{3}$ and $\mathrm{ND}_{3}$ targets by subtracting the corresponding carbon histogram (properly scaled to match with the shoulders from the background in each of the ammonia data). Since the carbon data is too low in counts (hence the raggedness in the histogram), a fit (a 'gaussian' times a 'linear' function) to the carbon data is obtained, and that fit (shown as the blue line in the first panel in Fig. 63 is used instead of the histogram itself to remove the background. The blue line in the second (top-right) panel and the cyan line in the last (bottom-right) panel show the properly scaled carbon fits which are subtracted from the $\mathrm{NH}_{3}$ and $\mathrm{ND}_{3}$ histograms (shown by red lines) respectively. After the subtraction, we get new histograms that represent 'pure' elastic or quasi-elastic data from protons and deuterons (shown by the magenta lines in the third and last panels respectively). 


\subsubsection{EXTRACTING THE CONTAMINATION}

After we have the 'pure' elastic or quasi-elastic data from $\mathrm{NH}_{3}$ and $\mathrm{ND}_{3}$ runs, we get the mean and the spread (standard deviation $\sigma$ ) of the proton elastic peak by fitting the $\mathrm{NH}_{3}$ data to a Gaussian function $f_{p}(x)$ (the blue line in the third panel with parameters $\mathrm{p} 0=$ height, $\mathrm{p} 1=$ mean and $\mathrm{p} 2=\sigma$ ). After we have the fit for the proton elastic peak, we fit the background subtracted deuteron data to a function $f(x)$ that is a linear combination of the pure proton fit and a pure deuteron fit (the latter with the form of a quadratic function $\times$ a Gaussian $^{8}$ ) as follows:

$$
f(x)=p_{0} \cdot f_{p}(x)+\left(p_{1}+p_{2} \cdot x+p_{3} \cdot x^{2}\right) \cdot e^{-0.5 \cdot\left(\frac{x-p_{4}}{p_{5}}\right)^{2}}
$$

where $p_{i}(\mathrm{i}=0,2, . ., 5)$ are the free parameters which are determined by fitting of $\mathrm{f}(\mathrm{x})$ to the deuteron data. The first term $p_{0} \cdot f_{p}(x)$ in $\mathrm{f}(\mathrm{x})$ represents the contribution from the contaminant (i.e., protons in $\mathrm{ND}_{3}$ ) and the rest of the term in $\mathrm{f}(\mathrm{x})$ represents the contribution from the deuterons in $\mathrm{ND}_{3}$. The total fit function $\mathrm{f}(\mathrm{x})$, the proton contribution and the deuteron part are shown by the blue, green and black lines in the fourth panel. The ratio of the area under the green line to that under the blue line gives us the relative amount of the $\mathrm{NH}_{3}$ contamination in the $\mathrm{ND}_{3}$ target.

\subsubsection{RESULTS AND CONCLUSION}

From the calculation as described above, the estimate for the $\mathrm{ND}_{3}$ contamination came out to be $4.4 \%$ It was not possible to do a similar analysis on the $1.3 \mathrm{GeV} \mathrm{ND}$ data, because the CLAS acceptance constraints did not allow for the coincident detection of $e$ and $p$ from the exclusive (quasi-)elastic events. The basic conclusion is that at $2 \mathrm{GeV}$, we cannot get a 'pure' Gaussian spectrum for deuteron, and therefore, there is no way to unambiguously separate deuteron from proton in $\mathrm{ND}_{3}$. The fact that the fit looks reasonably well (with contamination coming out to be only a few percent) and that we clearly do not see a narrow peak on top of a wider one (unlike in EG1-DVCS) should be sufficient to ascertain that EG4 did NOT have the same contamination problem as EG1-DVCS (which still has not been explained yet) [63]. To accommodate the fact that the contamination measurement is not reliably unambiguous, we will assume a rather generous systematic error due to the contamination.

\footnotetext{
${ }^{8} \mathrm{~A}$ pure Gaussian and other forms for the deuteron spectrum were tried but the overall fit was not as good.
} 


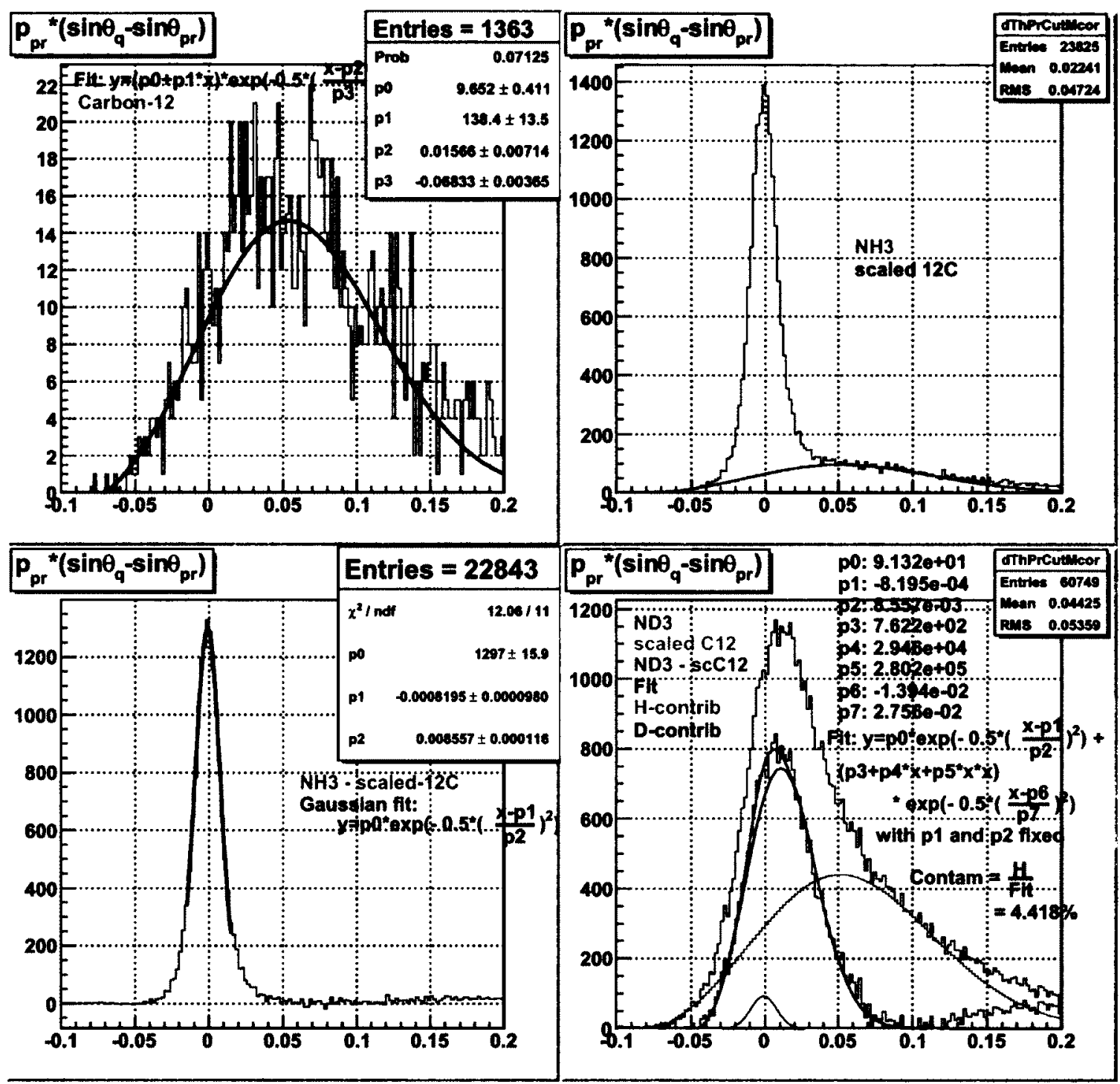

FIG. 63. Histograms showing the quantity $\Delta=p_{p} \cdot\left(\sin \theta_{q}-\sin \theta_{p}\right)$ for elastic or quasielastic events from carbon-12 (top-left), $\mathrm{NH}_{3}$ (top-right) and $\mathrm{ND}_{3}$ (bottom-right) target runs respectively. The third (bottom-left) panel shows the background removed elastic events from the $\mathrm{NH}_{3}$ data. In the fourth panel, various $\Delta$ are shown - red is the raw $\mathrm{ND}_{3}$, light green is the scaled- ${ }^{12} C$ for the nuclear background, brown is for the difference between the two. 


\subsection{SIMULATION AND APPROACH TO ANALYSIS}

The EG4 data consist of a table numbers of electrons reconstructed within various $\left(W, Q^{2}\right)$ bins that are scattered off polarized hydrogen $\left(\mathrm{NH}_{3}\right)$ or deuteron $\left(\mathrm{ND}_{3}\right)$, divided by the (life-time gated) integrated charge, for two different combinations of target polarization and beam helicity:

$$
n^{ \pm}=N^{ \pm} / F C^{ \pm}
$$

where "+" refers to beam helicity and target polarization anti-parallel, while "-" refers to the parallel case. The difference between these two normalized counts is given by

$$
\Delta n\left(W, Q^{2}\right)=n^{+}\left(W, Q^{2}\right)-n^{-}\left(W, Q^{2}\right)=\mathscr{L}_{r} \cdot P_{b} P_{t} \cdot \Delta \sigma\left(W, Q^{2}\right) \cdot \operatorname{AccEff}\left(W, Q^{2}\right)+B g
$$

where the "relative luminosity" $\mathscr{L}_{r}$ is a constant factor containing the density of polarized target nuclei per unit area and the conversion factor from Faraday cup counts to integrated number of electrons incident on the target; $P_{b}$ and $P_{t}$ are the beam and target polarization, $A c c$ and $E f f$ are the geometric acceptance and detection efficiency of CLAS for electrons within the kinematic bin in question (including cuts and trigger efficiency), and the background $\mathrm{Bg}$ comes from several sources, including pions misidentified as electrons, electrons from $e^{+} e^{-}$pair production, and electrons scattered off (partially) polarized target nucleons and nuclei that are not the intended species (e.g., bound protons in ${ }^{15} \mathrm{~N}$, free proton contamination in nominal $\mathrm{ND}_{3}$ targets, and bound proton-neutron pairs in any ${ }^{14} \mathrm{~N}$ contamination present) ${ }^{9}$.

Our main goal is to extract the spin structure function $g_{1}$ and calculate its moments. The cross section difference $\Delta \sigma\left(W, Q^{2}\right)$ on the right side of the above equation is what contains the information on $g_{1}\left(W, Q^{2}\right)$ along with various other contributions. ${ }^{10}$ This means we can, in principle, calculate the cross section (and then use that to extract $g_{1}$ ), from the background corrected measured quantity $\Delta n\left(W, Q^{2}\right)$ by putting in the values for all the rest of the quantities involved in Eq. 131. But, in reality, having an accurate knowledge of $A c c$ and $E f f$ is challenging and the available measurements of polarizations and luminosities are not reliable enough. So, experimenters usually resort to Monte-Carlo simulation to

\footnotetext{
${ }^{9}$ While this background is a small correction for hydrogen targets, in the case of deuteron targets, it must be corrected for (see Sec. 4.16.1).

${ }^{10} \Delta \sigma\left(W, Q^{2}\right)$ also has contributions from the unmeasured $g_{2}$ or, equivalently, from the product $A_{2} F_{1}$. Moreover, the cross section receives modifications and tails from radiative effects (both internal and external radiation) and kinematic resolution smearing.
} 
determine some or all of those factors that are involved in the relation between the counts and cross-section differences.

A standard way to extract the sought-after Physics quantities from these kinds of measurements proceeds along the following steps [93]:

1. Use a full simulation of CLAS with a "realistic" event generator, detector simulation and event reconstruction including cuts to obtain the product $A c c E f f$ as the ratio of events reconstructed in a particular bin, divided by events thrown in that same bin.

2. Extract the product $\mathscr{L}_{r} \cdot P_{b} P_{t}$ from the ratio of the acceptance and efficiency corrected $\Delta n$ in the (quasi-)elastic region $(0.9<W<1.0)$ to the well-known theoretical cross section difference for elastic (or quasi-elastic) scattering off the proton (deuteron).

3. Estimate and correct for $B g$.

4. Apply radiative corrections, which use a model of the unradiated Born cross section and a calculation of the radiated cross section based on programs like RCSLACPOL (see below). There is some ambiguity in how to apply these corrections; e.g., one can attempt to separate the effect of the (quasi-)elastic (or other) tail which should be simply subtracted from the measured cross section difference, and a multiplicative factor that accounts for vertex corrections and all other effects not accounted for in the tail. In practice, one has to repeat the calculation of these radiative corrections several times with different model input and assumptions about the target, to assess systematic uncertainties.

5. Express the extracted Born cross section difference in terms of the desired quantity (here: $g_{1}$ ) and additional input (e.g., $A_{2} F_{1}$ ). Use a model for the latter to extract $g_{1}$ only. Vary the model (concurrently with the model input to the previous step) to assess systematic uncertainties.

One conceivable problem with this approach lies in the first step, and in particular with the choice of the "realistic event generator". This choice would not matter at all if two conditions are fulfilled [93]:

1. The kinematic bins are chosen so small that the variation of the cross section over the bin (and/or the corresponding variation of the acceptance times efficiency) do not lead to any significant deviations for the average AccEff between the simulation and the real detector. 
2. The counts reconstructed within any one bin are directly proportional to the number of initial electrons generated within that same bin (the proportionality constant being $A c c E f f$ ), without any "bin migration" from other kinematic bins. (Otherwise, the ratio reconstructed/generated depends on those "migration tails", and the simulation will give different results from the "true value" if the overall cross section model of the generator is not accurate enough.)

Unfortunately, assumption 1 tends to directly contradict assumption 2 because 1 favors small bins and 2 favors large bins! For most precision experiments, bin migration effects are significant. This is aggravated by the difficulty of making a clean separation between bin migration due to detector resolution alone and the contribution from radiative effects. For instance, GEANT and therefore GSIM includes (at least by default) photon radiation as part of the simulation of outgoing electron tracks throughout the gas and building materials of all detectors. It is very important not to "double count" when simulating an experiment; the radiative calculations in step 4 above should not include any "after" radiation beyond the limit of the target itself (which, in turn, should then NOT be included in the GSIM simulation as material to be traversed).

This is a problem for all CLAS experiments attempting to extract absolute cross sections (or, here, cross section differences); however, the problem is magnified for our case: Since the cross section difference itself is not required to be positive, one can have both positive and negative tails migrating into adjacent bins. In any case, it is clear that using the average, unpolarized cross section as a model for the generator is not really appropriate (unless one is confident that the asymmetry is fairly constant or slowly-varying - not a good assumption in the resonance region where the $\Delta(1232)$ with negative asymmetry is adjacent to the S11 with positive asymmetry). Using a (hopefully realistic) model of the cross section difference instead would be much better, but this causes two new problems [93]:

1. Prima facie it is unclear how to simulate a negative cross section (difference). This problem can be circumvented fairly easily (see below), albeit at extra cost in terms of simulation effort.

2. It obviously becomes impossible to extract $A c c E f f$ from a simple ratio of reconstructed divided by generated events; both of these quantities could be positive, negative (even different sign under extreme circumstances), or - particularly bad for the denominator - zero. From this discussion, it is also clear that such a ratio would 
depend very sensitively on the cross section model and bin migration tails and be a very poor indicator of the actual product $A c c E f f$.

For this reason, we decided to try a different approach outlined in the following. The basic idea is to study the dependence of the reconstructed count difference on the model input (in particular $g_{1}$ ) directly through the whole chain of simulation and reconstruction, and then use tables of Born and radiated cross section differences for various model inputs as estimates of systematic uncertainties ${ }^{11}$.

\subsubsection{OUTLINE OF THE METHOD}

The basic idea is the following: If we already had a perfect model of $g_{1}$ and all other ingredients that go into $\Delta n$ (including a perfect simulation of CLAS), a simulation of $\Delta n$ would agree $100 \%$ with the data (within statistical errors). Any (larger than statistical) deviation between such a simulation of $\Delta n$ and the data can only be due to the following possible sources:

1. The model for $g_{1}$ must be adjusted to reflect the "true" $g_{1}$. This is the default assumption which we will use to extract $g_{1}$ from the data. This will be done by finding the proportionality factor between small changes in $g_{1}$ and the reconstructed $\Delta n$ and then adjusting $g_{1}$ to fully account for the observed $\Delta n$.

2. There could be a systematic error on this proportionality factor (which, after all, will come from simulation); for instance, there could be systematic deviations from the simulated results for acceptance and efficiency (in particular efficiencies of the $\mathrm{CC}$, EC, or tracking, that are not perfectly simulated by GSIM). This is a multiplicative uncertainty that must be carefully estimated and applied to the final data.

3. Any imperfect simulation of the "background" due to all events not originating in the bin in question (migration, radiation), or due to undesired target components (hydrogen, bound polarized nucleons in nitrogen), or from misidentified pions or $e^{+} e^{-}$ pairs, or due to contributions to $\Delta \sigma$ from $A_{2}$ can lead to an additive systematic deviation that would then be misinterpreted as a change in $g_{1}$. This systematic uncertainty must be studied by varying model inputs, parameters etc. in the simulation.

\footnotetext{
${ }^{11}$ We developed this method for the case of an $\mathrm{ND}_{3}$ target; however, it could, of course, easily be adopted to $\mathrm{NH}_{3}$, as well
} 


\subsection{RADIATIVE CORRECTIONS}

The physics quantities that we seek to extract from measurements are theoretically defined or interpreted and calculated in terms of the cross-section of the so called "Born" scattering process, which is represented by the simplest possible Feynman diagram i.e., by the lowest order approximation of a single photon exchange process. However, the measured cross-sections also contain contributions from higher order electromagnetic processes, which must be accounted for before extracting the quantities of our interest. These additional contributions are grouped into two categories - internal and external radiative corrections.

The internal corrections are the contributions from the higher order QED processes (higher order Feynmann diagrams) which occur during the interaction. These include the correction for the internal Bremsstrahlung (i.e., the emission of a real photon while a virtual photon is being exchanged with the target) by the incoming or the scattered electron), the vertex correction (in which a photon is exchanged between the incoming and the scattered electron), and the correction for the vacuum polarization of the exchanged virual photon $\left(e^{+} e^{-}\right.$loops).

External corrections include those that account for the energy loss (mainly by the Bremsstrahlung process) of electrons well before or after the interaction while passing through the target material and the detector.

If the beam electron radiates a photon before the scattering, the kinematics of the actual process will be different from the the one calculated with the nominal beam energy. Likewise, if the radiation occurs after the scattering, the actual energy and momentum of the scattered electron will be different from what is calculated normally (i.e., without any radiation). The effect can be quite large for elastic scattering.

\subsection{3 "STANDARD" SIMULATION}

The simulation process consists of mainly three parts - generating events similar to the ones as produced in the double polarization scattering process, simulating the CLAS detector response, and finally the event reconstruction from the simulated detector signals.

The first part is accomplished by using a program that is made by combining the essential elements of an updated version of the "RCSLACPOL" program (for cross section generation) and some parts of the "STEG" event generator (see sections 4.13.1 and 4.13.2). 
The second part is done by two standard CLAS software packages running in succession - "GSIM" and "GPP"(see sections 4.13.3 and 4.13.4). And, finally, the standard CLAS package "RECSIS" is used to reconstruct the events in the same way as for the real data.

\subsubsection{RCSLACPOL}

The simulation for the standard model cross sections proceeds as follows. We use the code "RCSLACPOL" [94] that can generate polarized and unpolarized cross sections (both Born and radiated) based on the approach by Shumeiko and Kuhto [95] as well as Mo and Tsai [96], including external radiation in the target. This code has been extensively tested and used for the analysis of SLAC experiments E142, E143, E154, E155 and E1 55x as well as Jefferson Lab experiments like EGla and EGlb. It has been updated with the most recent models on polarized and unpolarized structure functions $\left(F_{1}, F_{2}, A_{1}\right.$ and $\left.A_{2}\right)[97,94,98,99]$ and an implementation of the folding algorithm developed by $W$. Melnitchouk and Y. Kahn [54] for structure functions of the deuteron. The models have been fitted to and tested with data from EG1b as well as world data on both $A_{1}$ and $A_{2}$ over a wide range of $Q^{2}$ and $W$, including the resonance region and the DIS region.

For EG4, we have "married" the "RCSLACPOL" code with that of the event generator "STEG". This generator uses a grid of (radiated) cross sections generated by our modified version of RCSLACPOL to generate events that are distributed according to these cross sections (i.e., the number of events generated in a given bin is proportional to the cross section integrated over this bin).

\subsubsection{EVENT GENERATOR}

The concept and some part of the generator skeleton was inherited from the STEG (SimplesT Event Generator) program obtained from INFN, in Genova, Italy. The old event sampling part (which made the program run extremely slow) of the code was replaced by a new one developed by myself which made the event generation process much faster. The cross section calculating part was replaced by codes from an updated version of RCSLACPOL (see Sec. 4.13.1).

The generator works in two stages. In the first stage, it generates two two-dimensional maps or tables of radiated inclusive polarized cross differences (for the scattering of polarized electrons from a longitudinally polarized deuteron target, by using RCSLACPOL) in various kinematic bins encompassing the kinematic region covered by EG4 data. These 
cross section maps (and the corresponding events later on) were generated in the following angular and momentum ranges: 5.0-45.0 degrees for $\theta, 250.0-325.0$ degrees for $\phi$ (to ensure the CLAS 6th sector is completely covered) and $\left(0.2, E_{\text {beam }}\right) \mathrm{GeV}$ for the momenta, where the beam energy $E_{\text {beam }}$ took values of 1.337 and $1.993 \mathrm{GeV}$, corresponding to the two $\mathrm{ND}_{3}$ data sets of EG4. In our case, the map was created by dividing the kinematic phase space into a grid of small rectangles and then calculating the differential crosssection at the geometric center of each of those squares (such as ABCD in Fig. 64). For our application, we need to generate two such maps (because of the impossibility of generating events according to negative cross-sections) and run the program twice - once corresponding to positive polarization and the next for the negative one. For all bins in which the integrated cross section $\Delta \sigma \geq 0$, we fill the first table ("positive map") which is therefore positive-definite. For all bins in which this cross section is below 0 , we fill a second table ("negative map"), but with the absolute (i.e. negative) value of this cross section.

In the second stage, events are thrown according to the cross section maps produced in the first stage. The events are given the vertex coordinates that are uniformly distributed over the volume of a $1 \mathrm{~cm}$ long cylinder with radius $0.01 \mathrm{~cm}$ around the beam line - with the center of this volume being at the EG4 target position of $(0,0,-100.93 \mathrm{~cm})$. Nearly equal numbers of events are generated for each polarization, they are finally normalized according to their total cross sections (integrals of the corresponding maps).

The kinematic and other information (positions, momenta, charge) of these generated events are recorded and saved in the BOS format ${ }^{12}$ output files which organizes data into banks. In our case, HEAD, MCEV, MCTK, and MCVX banks are used for the generator output. The generator is also capable of producing output in the hbook format which makes it possible to study the Monte Carlo data using PAW (or ROOT because the h2root program easily converts "hbook" files into "root" files).

\subsubsection{GSIM - CLAS DETECTOR SIMULATION}

The Monte Carlo events thus generated are next fed into GSIM - the CLAS Monte Carlo simulation program using GEANT 3.21 libraries from CERN [100]. It simulates the CLAS detector response by implementing a complete model of the detector as well as the propagation of particles through different materials including all physics processes, such as multiple scattering, energy loss, pair production, and nuclear interactions. The

\footnotetext{
${ }^{12}$ Existing versions of GSIM, GPP and RECSIS accept only BOS format for input files.
} 


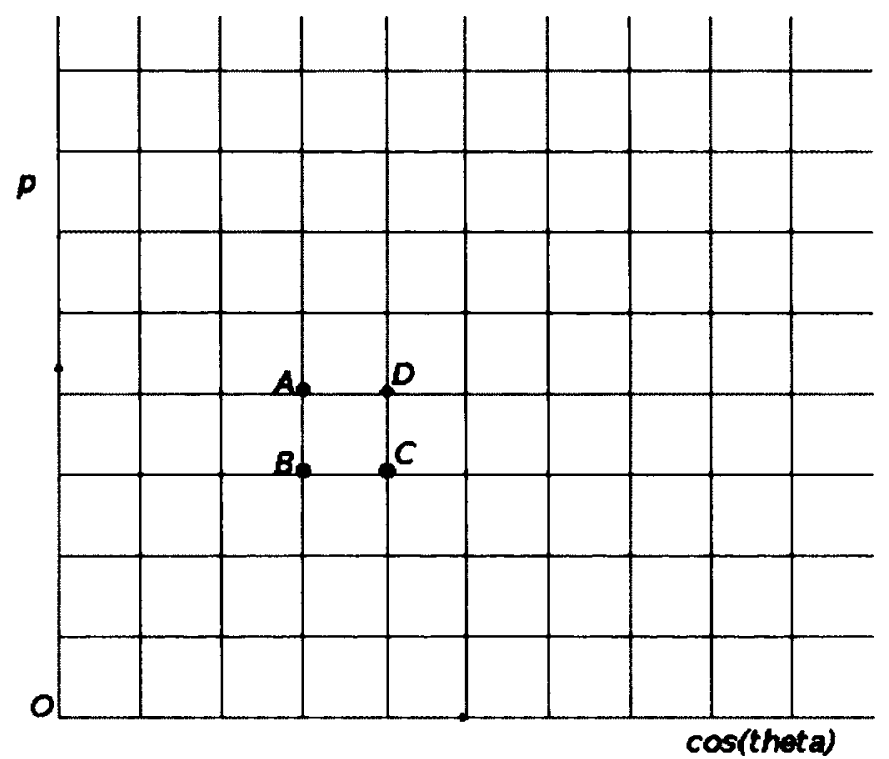

FIG. 64. Corners of a typical bin highlighted in the kinematic space covered by the event generator.

program takes the input event particles and then, based on their types, momenta and positions, "swims" (traces) them through all volumes of different materials that are defined using various library routines and the detector parameters. Charged particles are also subjected to the effects of the torus and target magnetic fields of the same strength as in the actual experiment (for this the same field maps are used as in the track reconstruction process using RECSIS). All the ingredients of the program (field maps, active detection volumes, passive volumes of detector support structures etc) are modeled as accurately as possible with the help of engineering designs and actual detector measurements. Special subroutines corresponding to various active parts of the detector produce outputs resembling the real detector signals which can then be reconstructed and analyzed just as the real experimental data [73][101]. GSIM is configured to match with the conditions of a given experiment by giving it proper values of input parameters via a command line input file which contains various "ffread cards" some of which are listed in table-2 along with their values that were used in our simulation.

\subsubsection{GSIM POST PROCESSOR (GPP)}

The GSIM output is next passed onto GPP - another standard CLAS software package 
- to process the simulated data further so that the detector response is accounted for more accurately. This package improves the response by smearing the detector signals and removing them if there are dead regions (determined by querying a data base which in turn is made by looking at the raw data of the experiment).

A lot of known, unknown, quantified, and unquantified factors such as temperature, alignment, dead channels, electronic malfunction etc affect the performance of the CLAS detector. But, GSIM does not include all these effects and, hence, the efficiency of the detector is always less than what the simulation provides us. To make the simulation more realistic by taking into account some of those effects, another CLAS software called GSIM Post Processor (GPP) is used to process the GSIM output. The GPP can change the DC, $\mathrm{SC}, \mathrm{CC}$ and $\mathrm{EC}$ signals produced in the simulation. The $\mathrm{DC}$ signals can be changed by (a) accounting for the dead wires according to the calibration database, (b) shifting the DOCA mean value, and (3) smearing the hit signals according to the resolution determined by the calibration database or according to the command line input. Likewise, SC signals can be changed with a parameter input for smearing the time resolution. And, for the $\mathrm{CC}$ and $\mathrm{EC}$ signals, the GPP can use the hardware thresholds[102].

As the experimental conditions and detector configurations can change from one experiment to another, in order to run the GPP, we must have our own experiment specific calibration constants and parameters such as the run number $(\mathrm{R})$, the DC smearing scale values for regions 1,2 and $3(a, b, c)$ and the SC smearing scale value (f). Even for a given experiment, these constants and parameters are determined to be different for different data sets (corresponding to a given beam energy, for example). The value for $\mathrm{R}$ can be any run number belonging to a specific data set. This number is used to identify the entry of the calibration constants in the database that corresponds to the given data set. In order to simplify the job, we decided to use the timing resolutions determined by the calibration database assuming that they are good enough and need only to determine new values for the DC smearing. To further simplify the job, we assumed that the three DC Regions had identical resolutions, so the DC smear parameters $\mathrm{a}, \mathrm{b}$, and $\mathrm{c}$ would have the same values, and the common DC-smear value is what is determined from the procedure described below.

In order to determine the DC-smear, we generated a statistically significant number (about half million) of elastic-electron events distributed according to the elastic cross section and then ran them through GSIM, GPP and RECSIS. The pure proton target events, 
turning off the radiative effects are generated using the existing STEG event generator.

The simulated elastic events are then fed into GSIM, GPP and RECSIS, with GSIM and RECSIS used in the same configuration as when processing the CLAS data during the "pass-1" phase, and GPP run with different values of DC-smear scales as inputs. The reconstructed data coming out of RECSIS corresponding to a given value of DC-smear is then histogrammed in $\Delta \mathrm{E}$ again and fitted to a Gaussian to get its $\sigma$ (characterizing width) of and mean (characterizing position). As we can see in figures 4.65(a) and 4.65(b), the width of the elastic peak increases with the DC-smear but the position stays more or less the same as expected. In fact, when the two are plotted against DC-smear (as in figures 4.66(a) and 4.66(b)) the width shows a linear dependance.

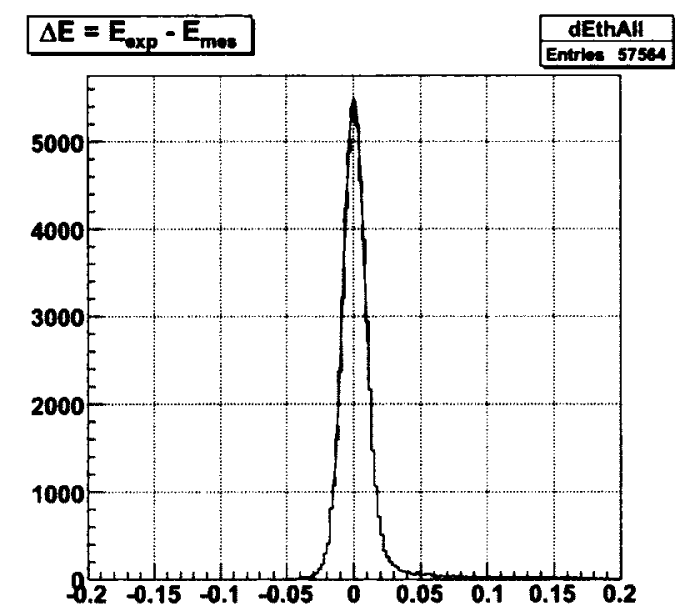

(a) Dc-smear scale $=1.3$

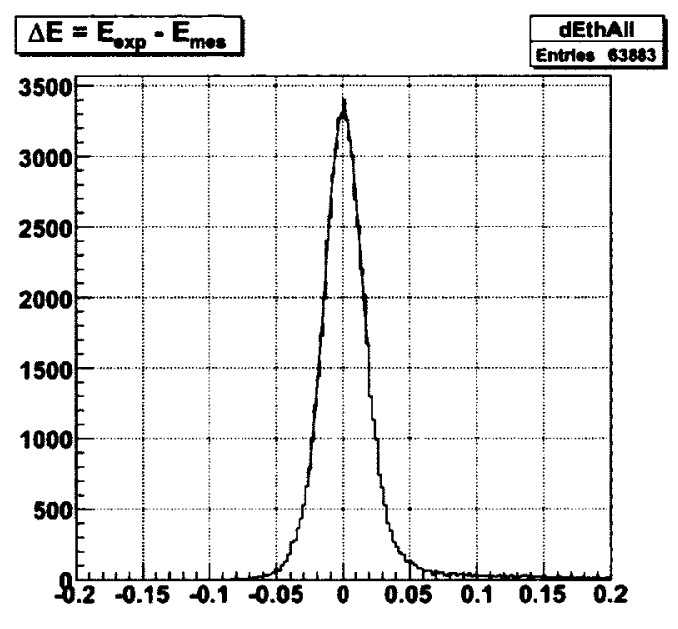

(b) Dc-smear scale $=2.9$

FIG. 65. $\Delta \mathrm{E}$ of $2.3 \mathrm{GeV}$ simulated elastic-only proton-target events passing through GSIM, GPP (with two different Dc-smear scales), and RECSIS.

\subsubsection{FINDING THE WIDTH OF THE REAL CLAS DATA ELASTIC PEAK.}

With the knowledge of the DC-smear dependence of energy resolution (Fig. 4.66(a)), if we also know the resolution in the real data, we can determine the right value of DCsmear which would make make the resolution in the simulation comparable with that in the real data. So, the next step is to find the resolution in the real CLAS data, which is done again by measuring the width of the elastic peak in the real data. But, because 


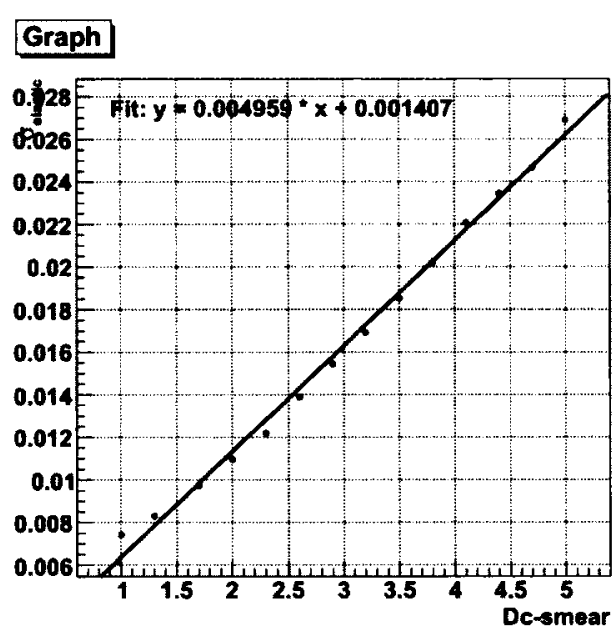

(a) $\sigma$ vs DC-smear

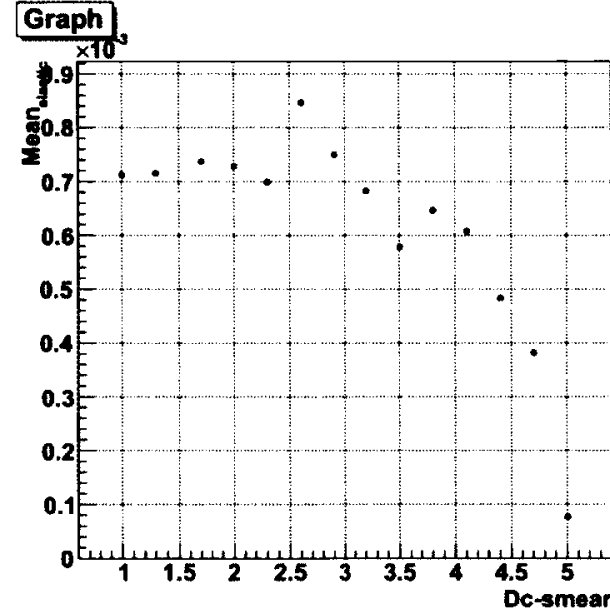

(b) Mean vs DC-smear

FIG. 66. Graphs showing the dependence of width and position (obtained from the Gaussian fits as shown in the fig (4.13.4) of the elastic peaks on the DC-smear applied to GPP.

the real data is a very complex mixture of events coming from various reaction channels, we must first have a way to separate the elastic data from the rest. One method entails histogramming $\Delta \mathrm{E}$ from both the $\mathrm{NH}_{3}$ and ${ }^{12} \mathrm{C}$ target data (for a given beam energy) and subtracting the latter (after the cross-normalization) from the former (as in fig (67)) to effectively remove the contribution from nitrogen component of the $\mathrm{NH}_{3}$ target leaving the contribution coming only (mostly) from the proton component. Another method consists of using only the $\mathrm{NH}_{3}$ data but this time calculating the helicity dependent cross-section difference in the elastic region Fig. (68). In the latter method, the difference removes the contribution from the unpolarized nuclear background because they have the same contribution to the opposite helicity state cross-sections. After the elastic data is separated, its $\Delta \mathrm{E}$ distribution is fitted to a Gaussian as with the simulation data and we arrive at the experimental energy resolution.

Using the first of the two methods mentioned above, the real data resolutions were evaluated for three different polar angle $(\theta)$ cuts - all $\theta$ (in fact $\theta \geq 7^{\circ}$ ), $\theta>15^{\circ}$, and $\theta>20^{\circ}$. The dependence of these experimental resolutions on the beam energy for these cases are shown together in the Fig. 69, along with the resolution for the case "all $\theta$ ", but determined from the cross-section difference method. Likewise, as described above, the DC-smear dependence of the simulated resolution were determined separately for all these three cases of angle cuts, so that we could compare the experimental resolutions with the 


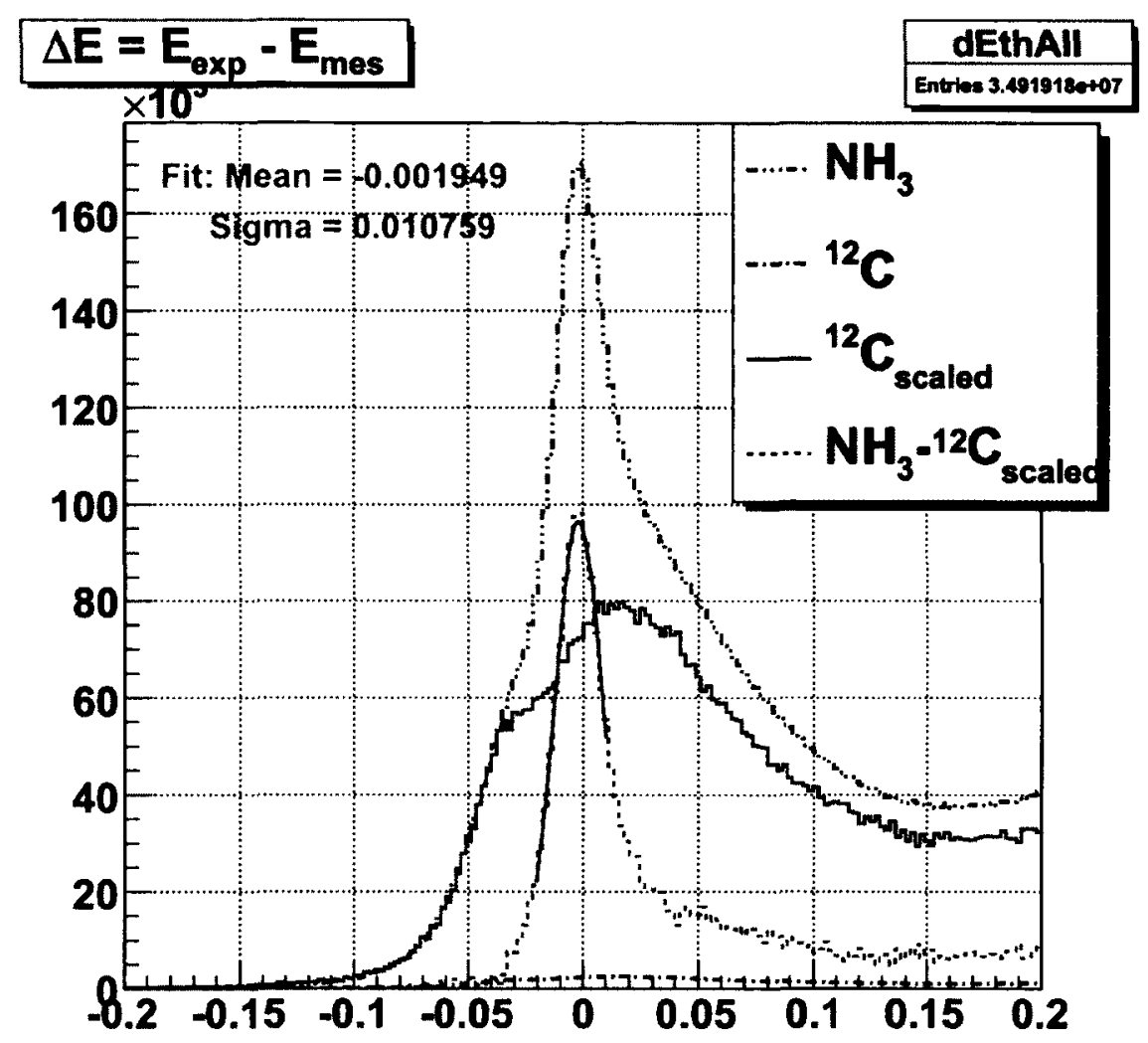

FIG. 67. Histograms illustrating the extraction of elastic peak for $2.3 \mathrm{GeV}$ by using carbon12 data for background removal from the total-cross section (all good electrons with $\theta>7$ used).

simulations correspondingly. One such comparison is illustrated in the figure 70 , where we show resolutions evaluated for the case of "all $\theta$ " - first two for the experimental data and the rest for the simulated data.

Looking at Fig. 69, it is obvious that the resolution is $\theta$-dependent as expected. When the experimental and simulated resolutions are compared for the three different cases of $\theta$ cuts, we realize that the GPP asks for the $\theta$ dependent DC-smearing, which makes the simulation work very complicated with the current version of GPP. To simplify the situation, we decide to have a global ( $\theta$ independent) value of DC-smearing (for a given beam energy) by comparing the experimental and simulated resolutions corresponding to the case of "all $\theta$ " cut. That should be good enough for practical purposes. By taking into account the fact that there seems to be an inherent uncertainty in the measurement of 

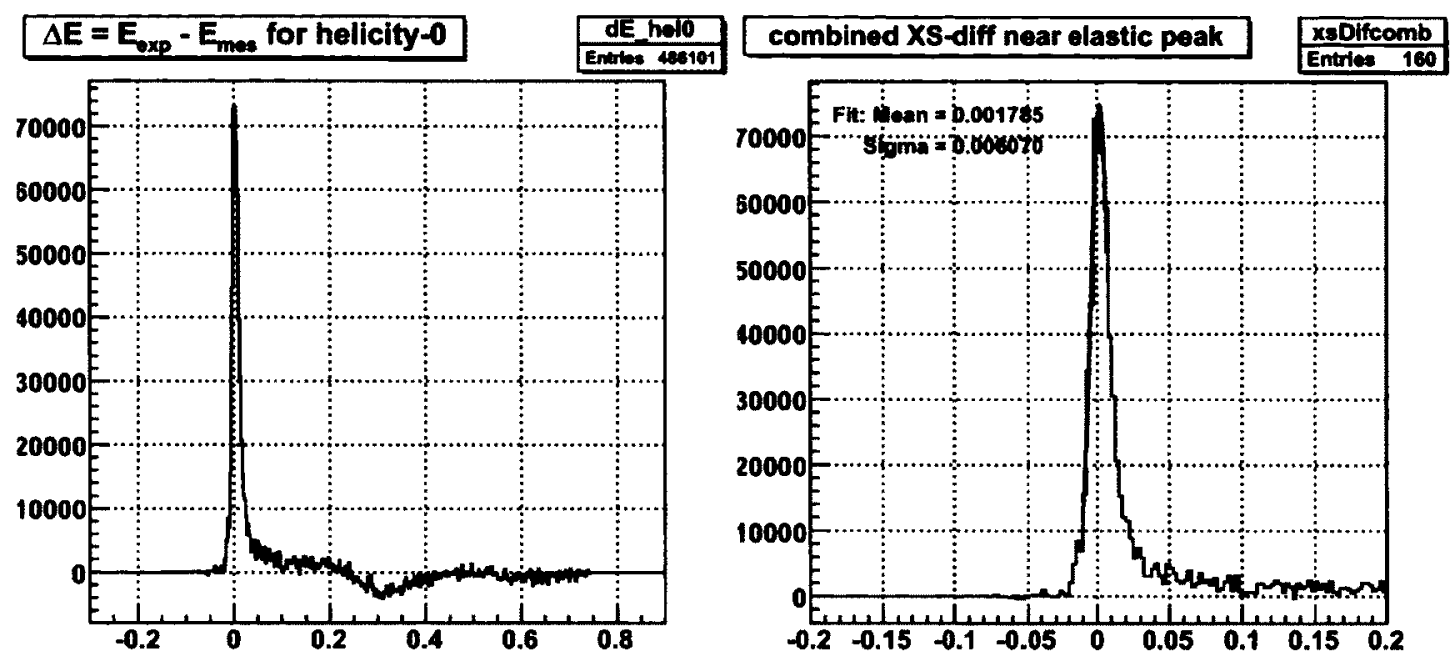

FIG. 68. Plots showing the cross-section difference for $2.3 \mathrm{GeV} \mathrm{NH}_{3}$ target data with the right one zoomed in around the elastic region (all good electrons with $\theta>7$ used).

the resolutions (evident from the discrepancy of the experimental resolutions determined from the two different methods) and comparing the experimental and simulated results, the values as listed in Table. 1 are chosen for the DC-smearing scales for the GPP. 


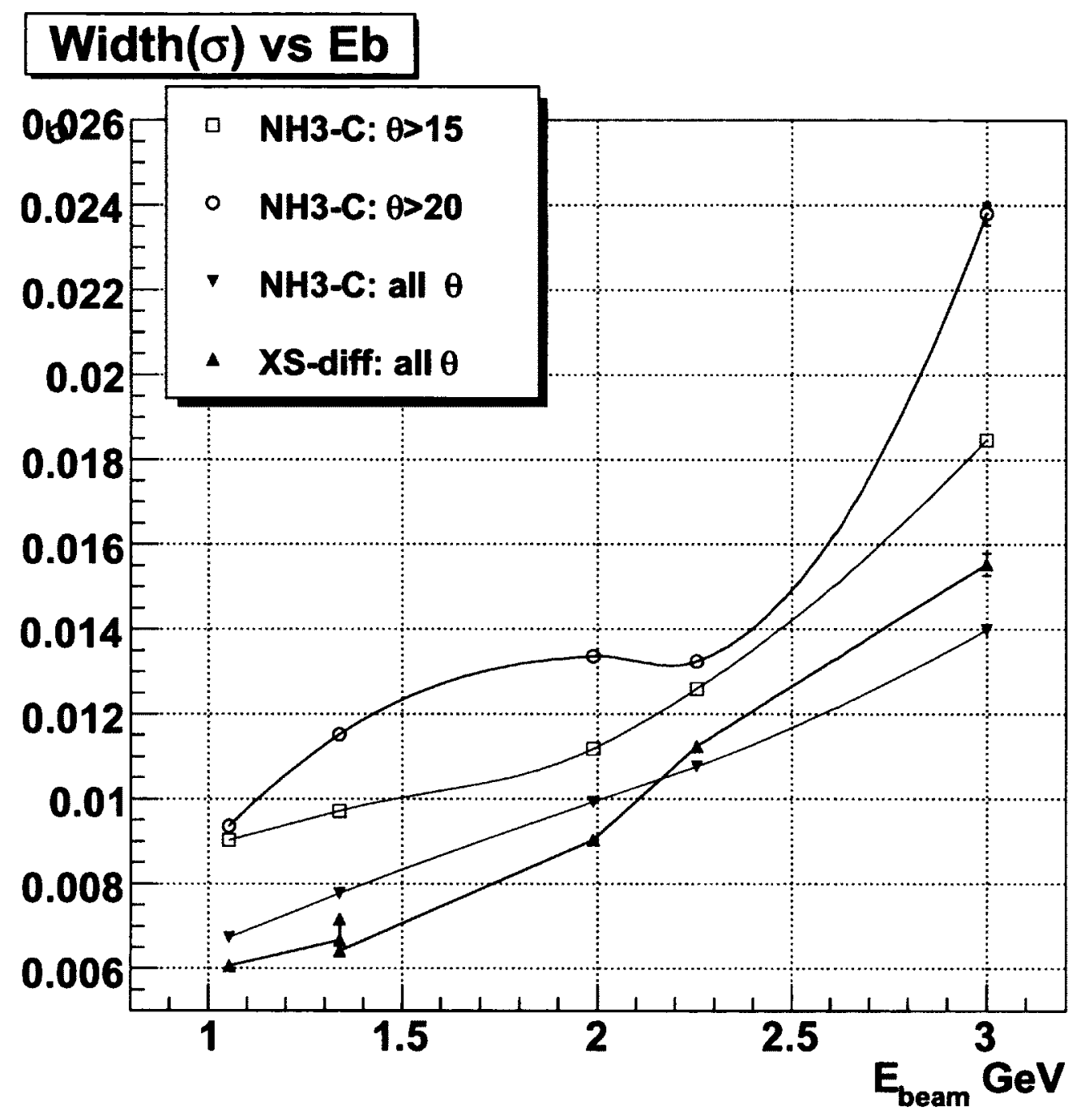

FIG. 69. Graphs showing the dependence of width $(\sigma)$ of the elastic peaks (from experimental data) on the beam energy $(\mathrm{GeV})$. 


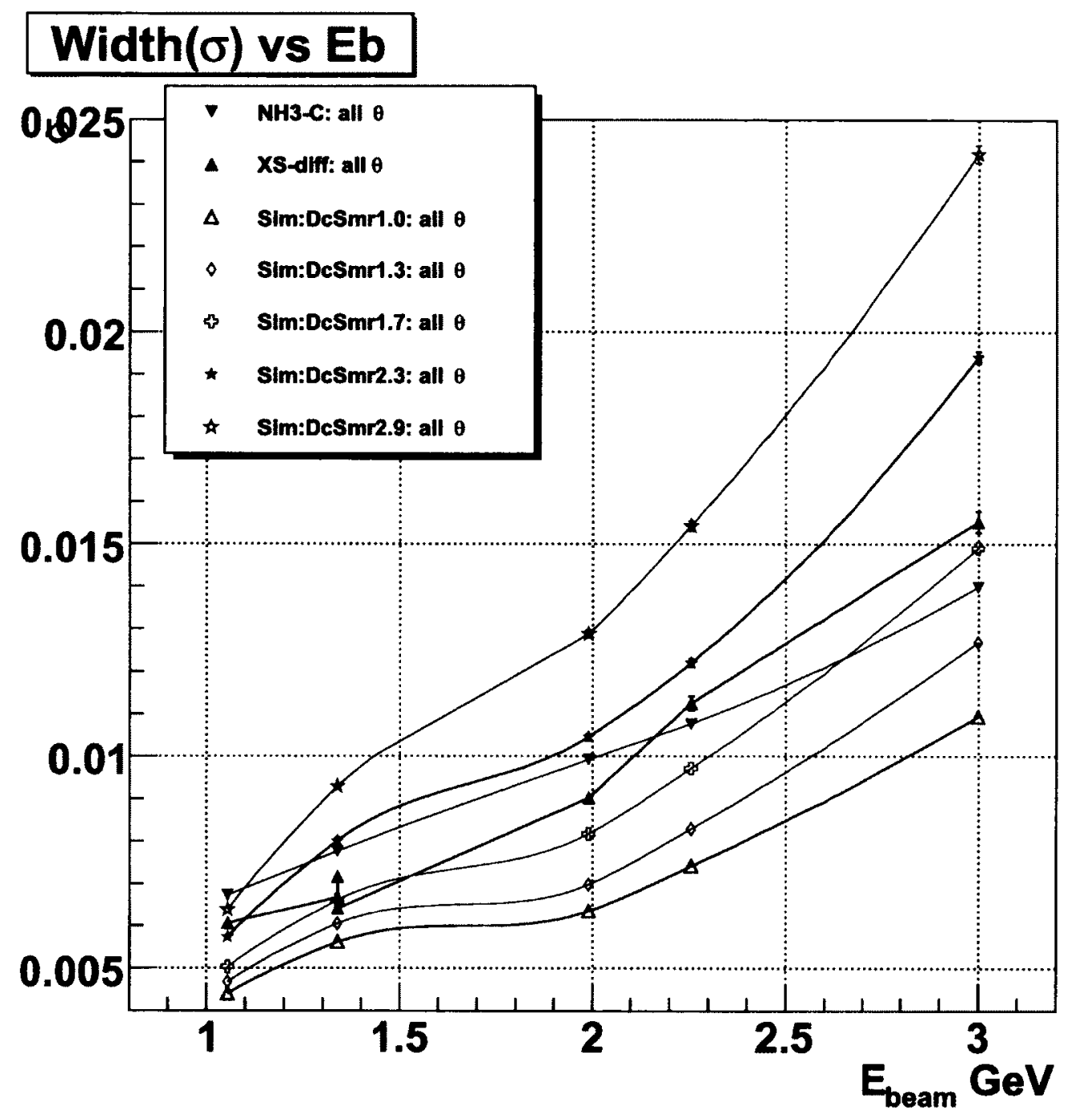

FIG. 70. Graphs showing the dependence of width $(\sigma)$ of the elastic peaks (from both experimental and simulated data) on the beam energy (GeV). 
TABLE 1. DC-smearing scales determined for different beam energies.

\begin{tabular}{|l|l|l|l|l|l|}
\hline$E_{\text {beam }}(\mathrm{GeV})$ & 1.054 & 1.339 & 1.989 & 2.256 & 2.999 \\
\hline DC-smear & 2.6 & 2.0 & 2.0 & 2.0 & 1.7 \\
\hline
\end{tabular}

\subsection{COMPARISON OF DATA AND SIMULATION}

Using our final values for the smear parameters, the simulated data were passed through GPP and then reconstructed with RECSIS. Finally, all applicable cuts and corrections were applied to both sets of polarized simulation data. Because the CC was turned of in GSIM for the simulation, all experimental data cuts except those depending on $\mathrm{CC}$ were applied to the simulated data. However, the cuts were modified (see Sec. 4.6) to account for differences between simulation and data.

In the end, we had two sets of simulated events (for the two cases of $\Delta \sigma \geq 0$ and $\Delta \sigma<0)$ in each kinematic bin. The number of these two type of events in each bin were then cross-normalized with respect to each other by their respective cross-section map integrals and the number of generated Monte-Carlo events and then combined to make the simulated polarized count difference $\Delta n$. To do that, the number of simulated event counts in a kinematic bin corresponding to the positive polarization was kept unchanged but the one corresponding to the negative polarization was multiplied with the following normalization factor:

$$
n o r m^{-}=\frac{\sigma_{t o t}^{-}}{\sigma_{t o t}^{+}} \times \frac{N^{+}}{N^{-}}
$$

where $\sigma_{t o t}^{+/-}$and $N^{+/-}$are the total integral of the cross section map and the corresponding number of Monte-Carlo events generated for each of the polarization cases $(+/)$.

The next step was to properly cross-normalize the simulated events to the data, as outlined in the introduction. For this, we found the scale factor $S F$ necessary to have the same $\Delta n$ in the quasi-elastic region (e.g., $0.9 \leq W \leq 1.0$ ). This factor represents the ratio

$$
S F=\frac{n^{+}-n^{-}}{\Delta n(\operatorname{simul})}
$$

since we assume that the simulation for the cross section difference in this region is reliable and all other factors are common to the simulation and the data. In fact, we chose one $Q^{2}$ bin (the $20^{\text {th }}$ one - for which the agreement between the data and simulation was among 

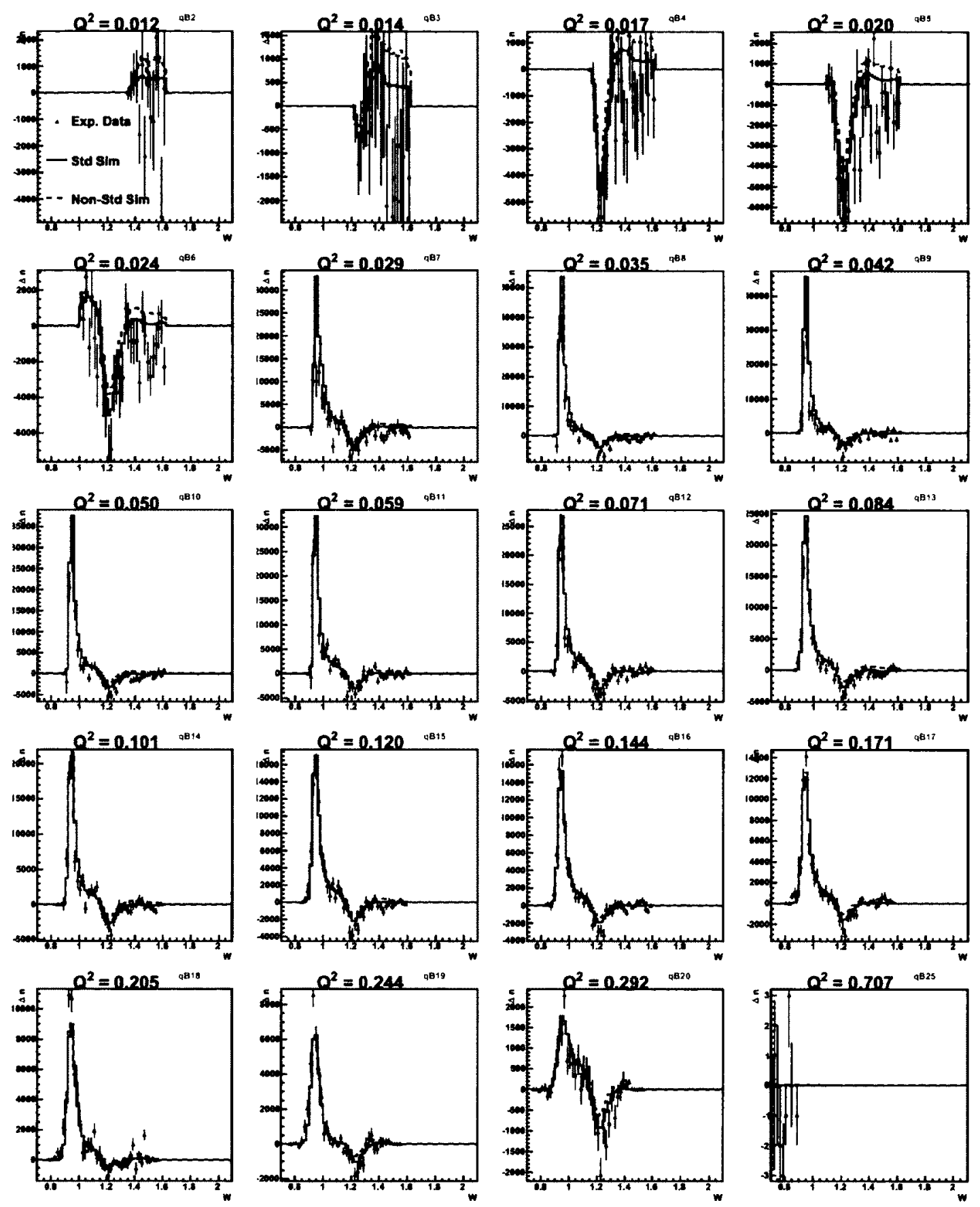

FIG. 71. Comparison (in different $Q^{2}$ bins) of polarized count differences from $1.3 \mathrm{GeV}$ experimental (red points with error bars) and two versions of normalized simulation data. The black continuous histograms are for "standard" simulation with values of $A_{1}$ set as given by the used model. The blue dotted histograms are for "non-standard" simulated data with $A_{1}$ changed to $A_{1}+0.1$. ). 

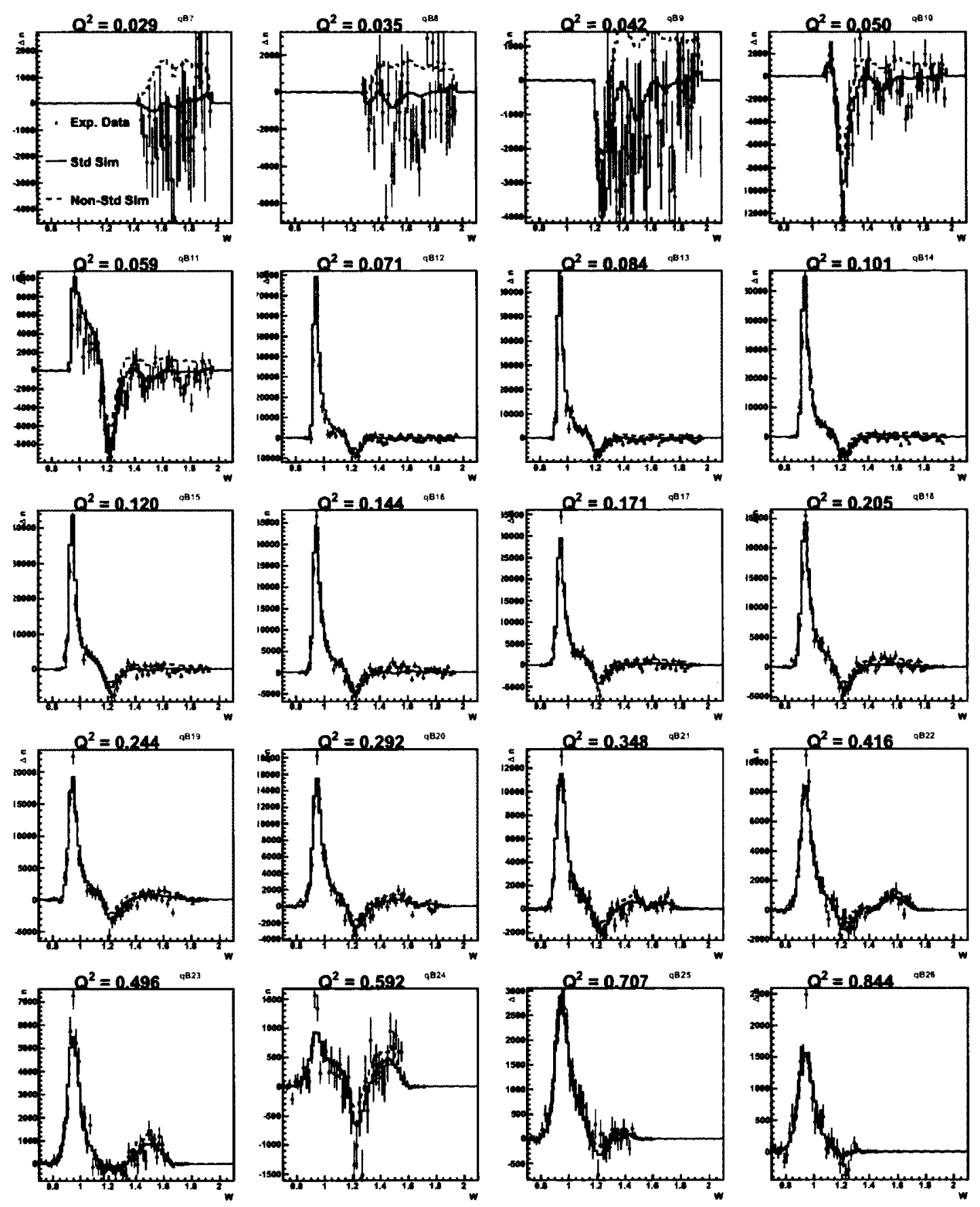

FIG. 72. Comparison (in different $Q^{2}$ bins) of polarized count differences from $2.0 \mathrm{GeV}$ experimental (red points with error bars) and two versions of normalized simulation data. The black continuous histograms are for "standard" simulation with values of $A_{1}$ set as given by the used model. The blue dotted histograms are for "non-standard" simulated data with $A_{1}$ changed to $A_{1}+0.1$. 
the best) and calculated above ratio to get the global preliminary value of the scaling factor $S F_{20}$. The simulated $\Delta n$ was then multiplied with this factor to get our best "prediction" of the real data in all the kinematic bins, in order to directly compare it with the real data (see Figs. 71 and 72).

After this normalization, the ratios $\left(n^{+}-n^{-}\right) / \Delta n(\operatorname{simul})$ in the quasi-elastic region for all $Q^{2}$ bins were calculated and plotted versus $Q^{2}$ as well as $\theta$ (see Figs. 4.73(a) - 4.76(a)) along with the corresponding statistical errors as given by $\sqrt{\left(n^{+}+n^{-}\right)} / \Delta n(\operatorname{simul})$. As the figures show, the ratio in the quasi-elastic region drops off rapidly at small $Q^{2}$. The fall-off is likely due to $\mathrm{CC}$ inefficiencies for very high momenta and very forward angles. Also, our simple cross section model for the deuteron is less accurate at low $Q^{2}$. Figs. 4.73(b) 4.76(b) show that the $\Delta$-resonance region does not suffer from similar problems.

The final normalization was obtained by calculating the error weighted average $S F_{\text {average }}$ of above ratios in the quasi-elastic region. The average was calculated using only those $Q^{2}$ bins which had ratios reasonably stable and closer to each other. Because, the ratios are reasonably stable only above $Q^{2} \approx 0.045 \mathrm{GeV}^{2}$ and $Q^{2} \approx 0.09 \mathrm{GeV}^{2}$ in the 1.337 and 2.0 $\mathrm{GeV}$ data sets respectively (as can be seen from Figs. 4.73(a) and 4.75(a)), only those $Q^{2}$ bins above these two limits were used in calculating the weighted average of these ratios. In addition, even above those two limits, some of those which had too large ratios - greater than 2.0 (or 2.5) for 1.337 (or 2.0) $\mathrm{GeV}$ data set- were not used in the weighted average. However, it should be noted that the bins not used in the average ratio calculations were not entirely discarded from the final analysis. Only those below $Q^{2}=0.02 \mathrm{GeV}^{2}$ were completely thrown out from the final analysis because they did not cover the resonance (particularly the $\Delta$ ) region very well. The resulting simulated data in the form of count differences $\Delta n$ in various $Q^{2}$ bins are shown in Figs. 71 and 72 along with the corresponding experimental data.

A complete systematic error analysis was done to study the effect of the overall scaling factor $S F$ on the extracted $g_{1}$ (see below) and to estimate its statistical (due to the number of counts) and systematic (due to model uncertainties and backgrounds) error. 


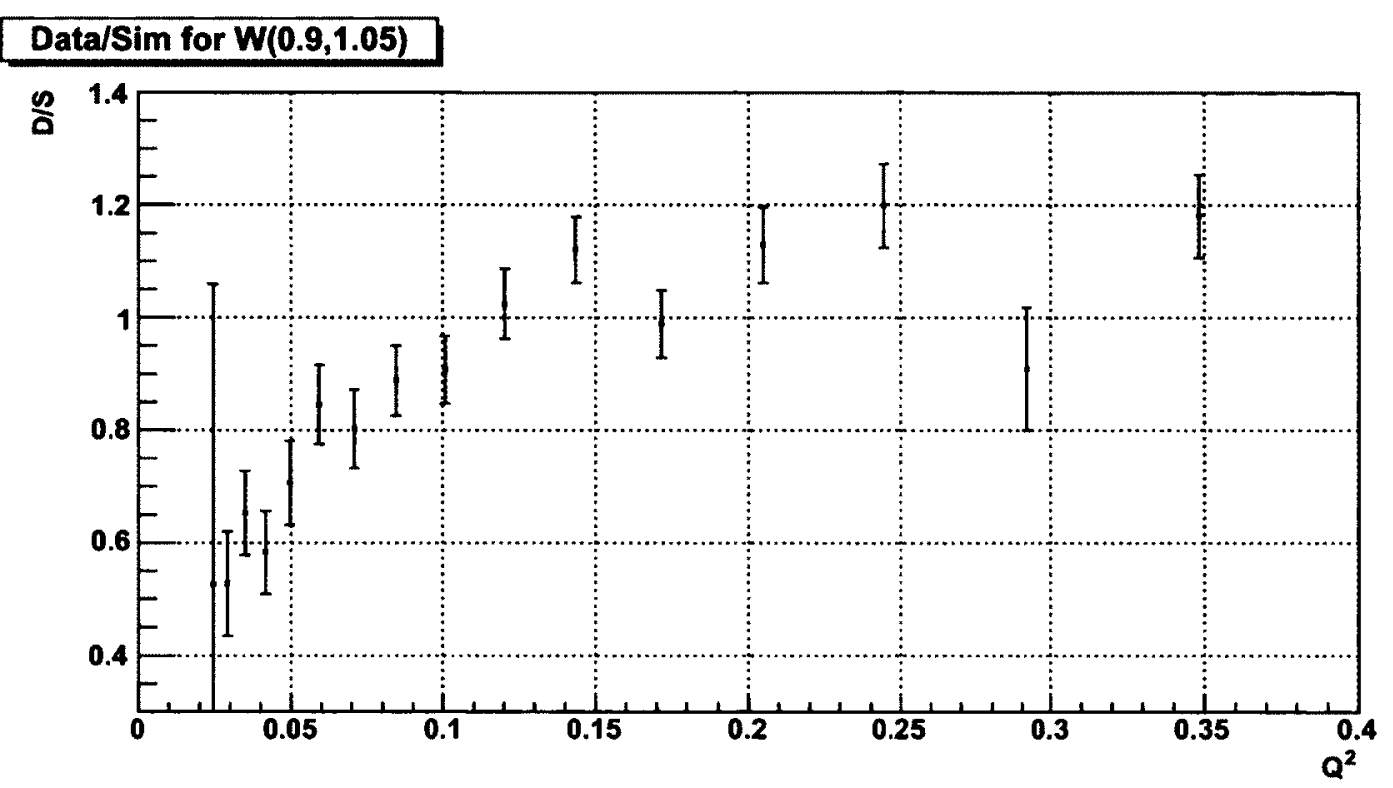

(a) Data/Sim ratio vs $Q^{2}$ in $1.3 \mathrm{GeV}$ quasi-elastic data.

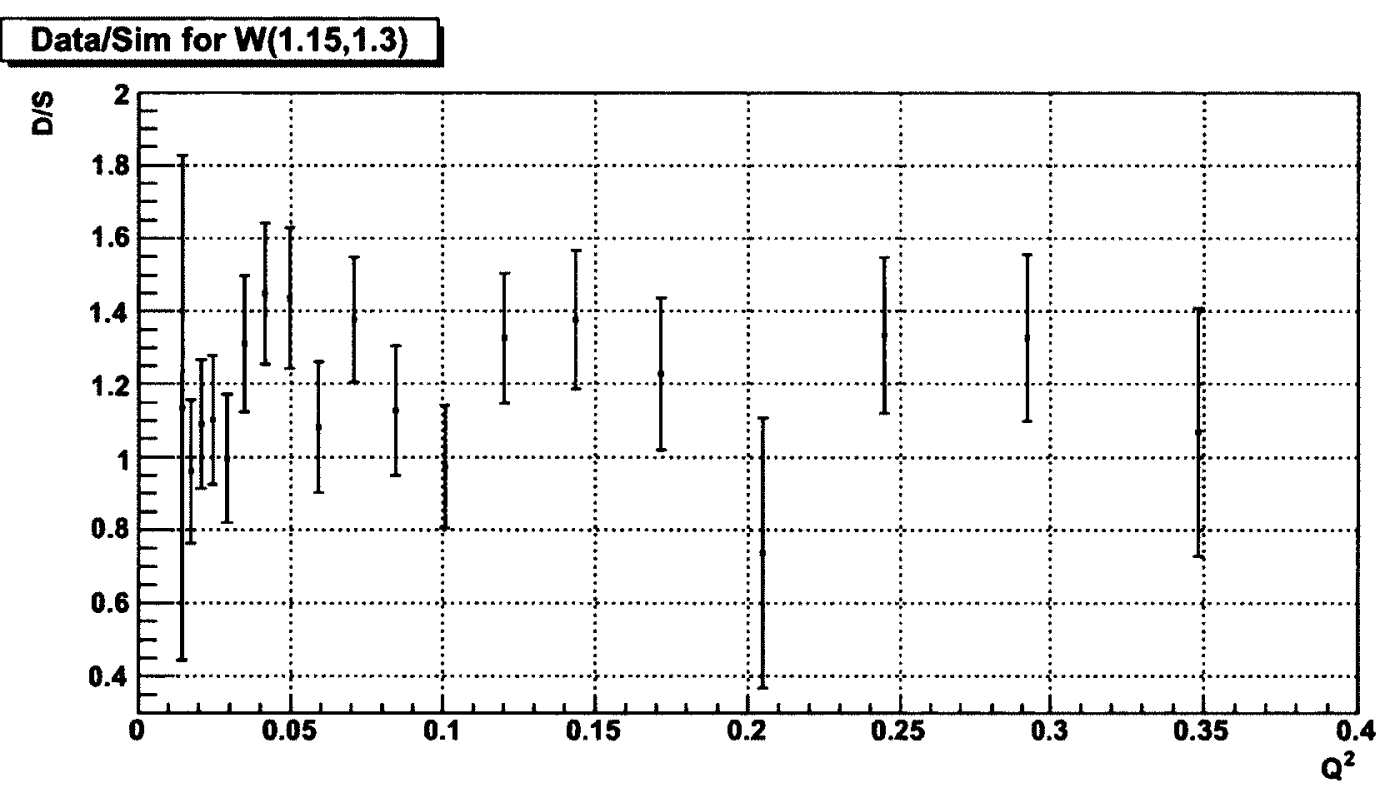

(b) Data/Sim ratio vs $Q^{2}$ in $\Delta$-resonance region of $1.3 \mathrm{GeV}$ data.

FIG. 73. $Q^{2}$ dependence of ratios of $1.3 \mathrm{GeV}$ data and simulation in the quasi-elastic and $\Delta$-resonance regions. 


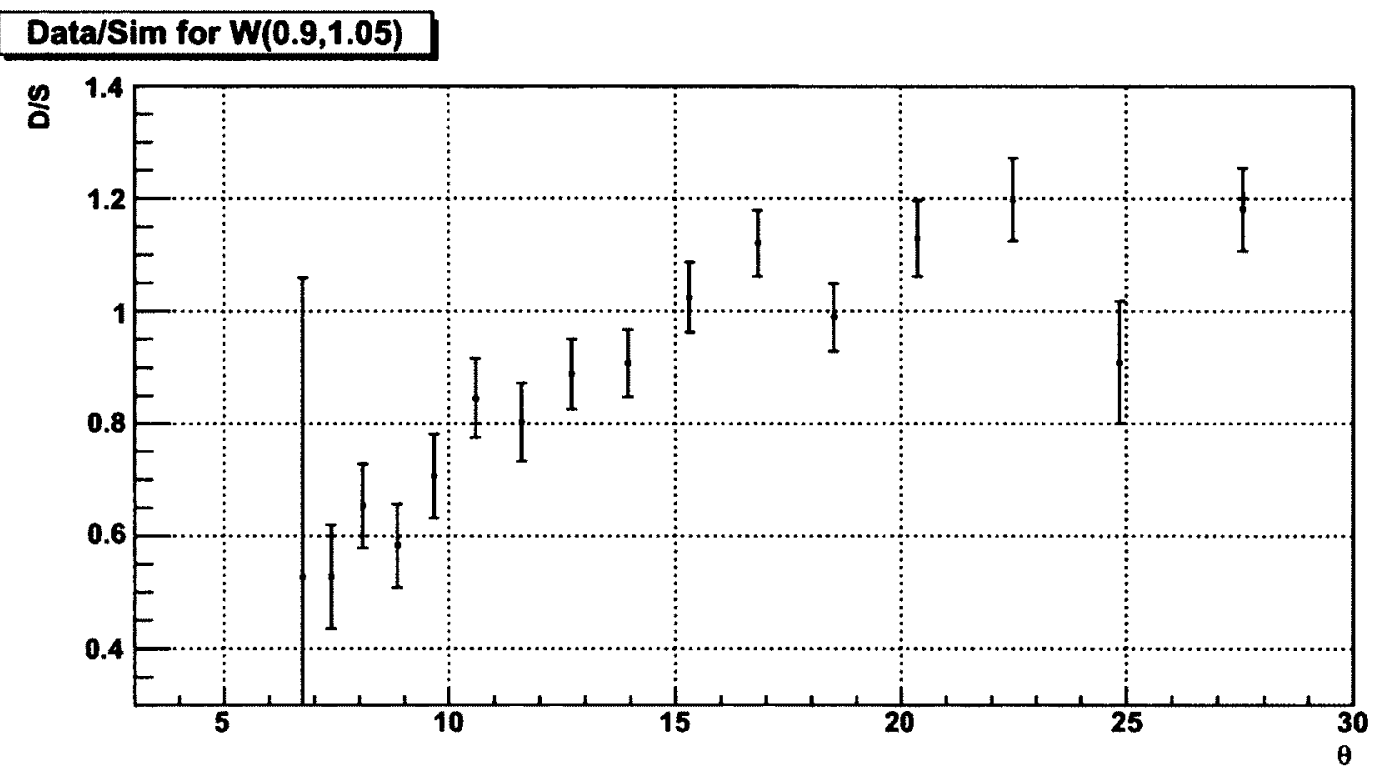

(a) Data/Sim ratio vs $\theta$ in $1.3 \mathrm{GeV}$ quasi-elastic data.

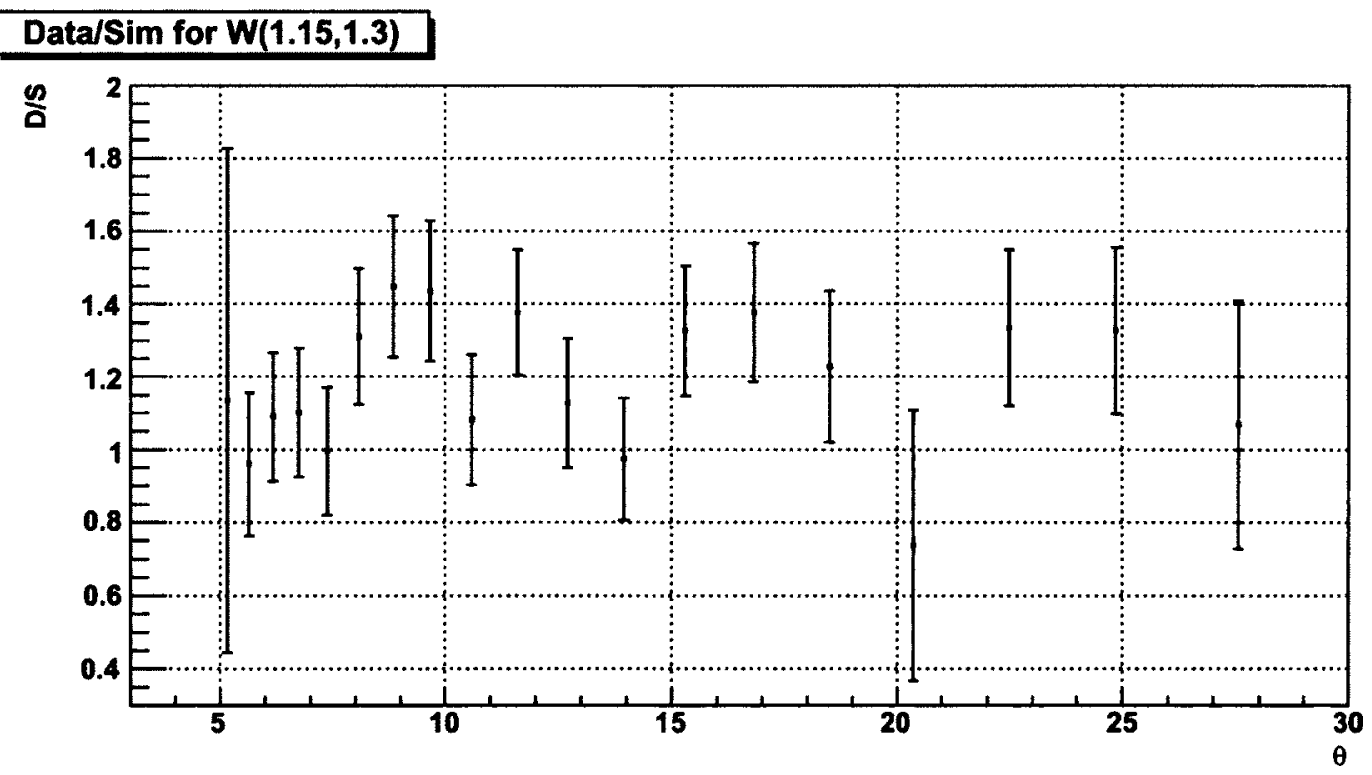

(b) Data/Sim ratio vs $\theta$ in $\Delta$-resonance region of $1.3 \mathrm{GeV}$ data.

FIG. 74. The same data as in Fig. 73, but plotted versus average scattering angle ( $\theta)$. 


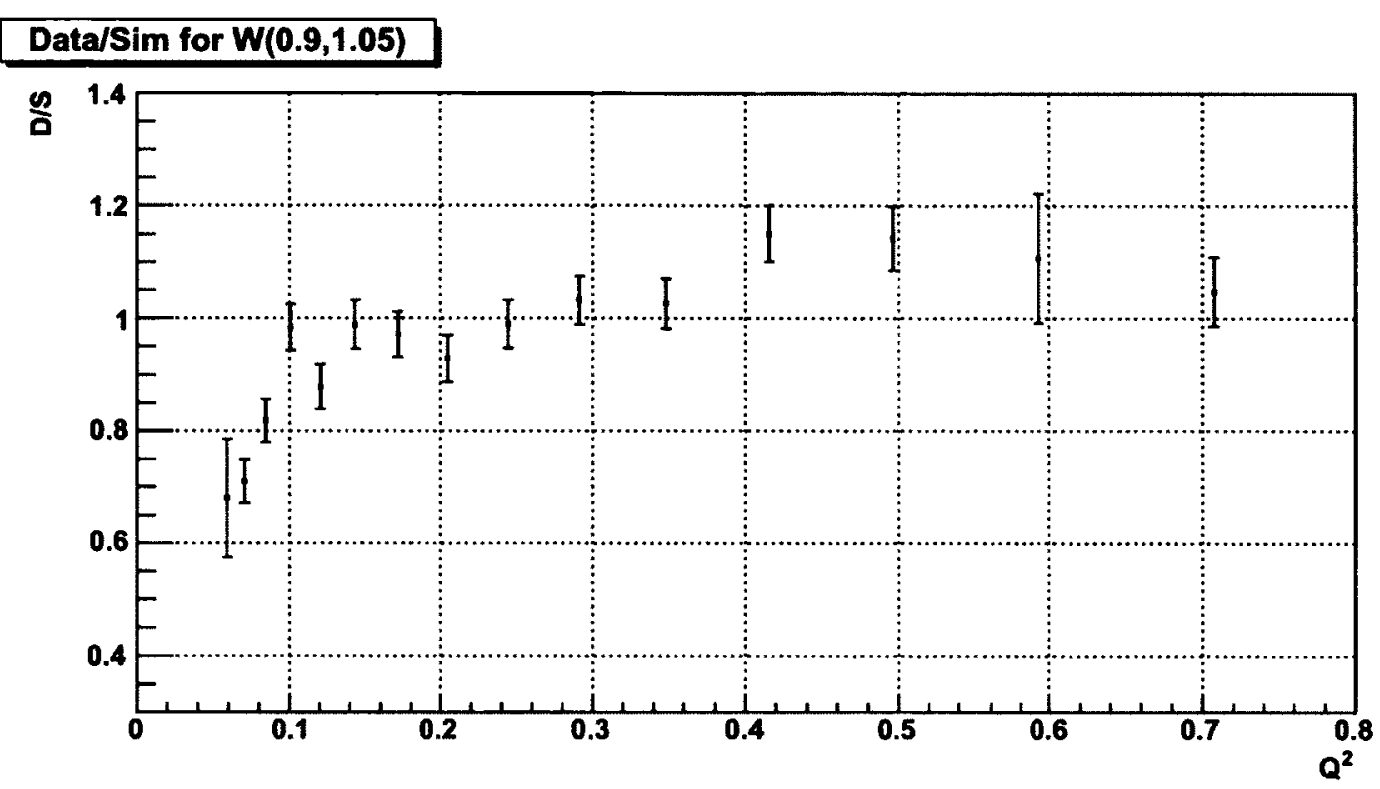

(a) Data/Sim ratio vs $Q^{2}$ in $2.0 \mathrm{GeV}$ quasi-elastic data.

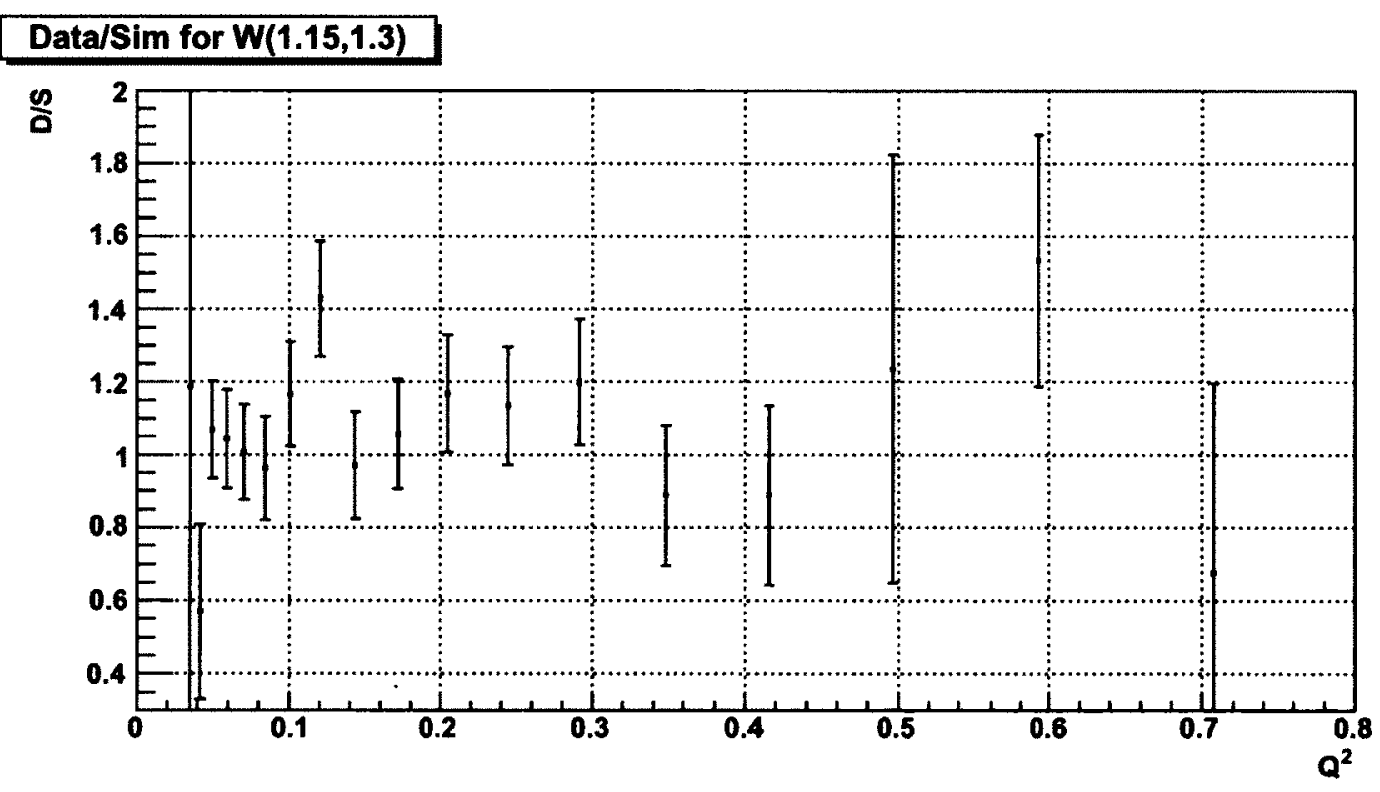

(b) Data/Sim ratio vs $Q^{2}$ in $\Delta$-resonance region of $2.0 \mathrm{GeV}$ data.

FIG. 75. $Q^{2}$ dependence of ratios of $2.0 \mathrm{GeV}$ data and simulation in the quasi-elastic and $\Delta$-resonance regions. 


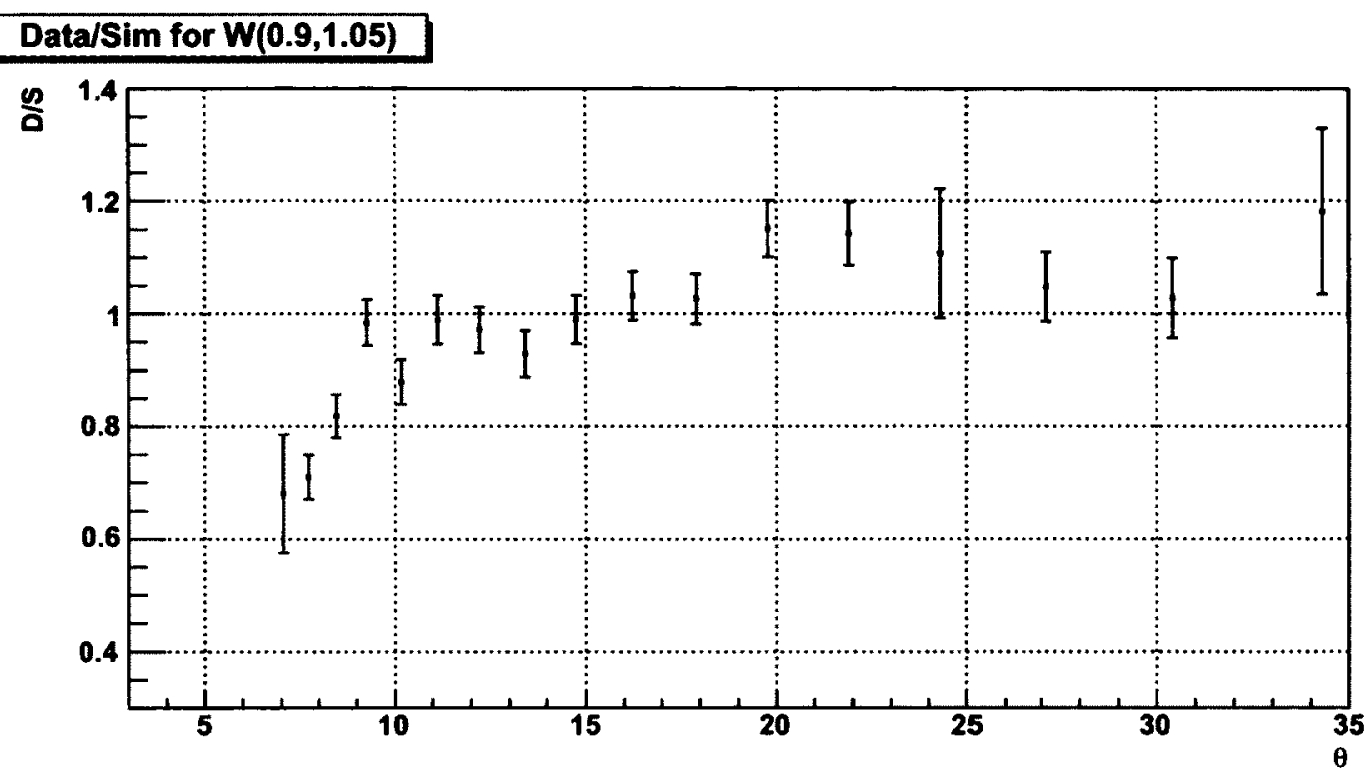

(a) Data/Sim ratio vs $\theta$ in $2.0 \mathrm{GeV}$ quasi-elastic data.

\section{Data/Sim for W(1.15,1.3)}

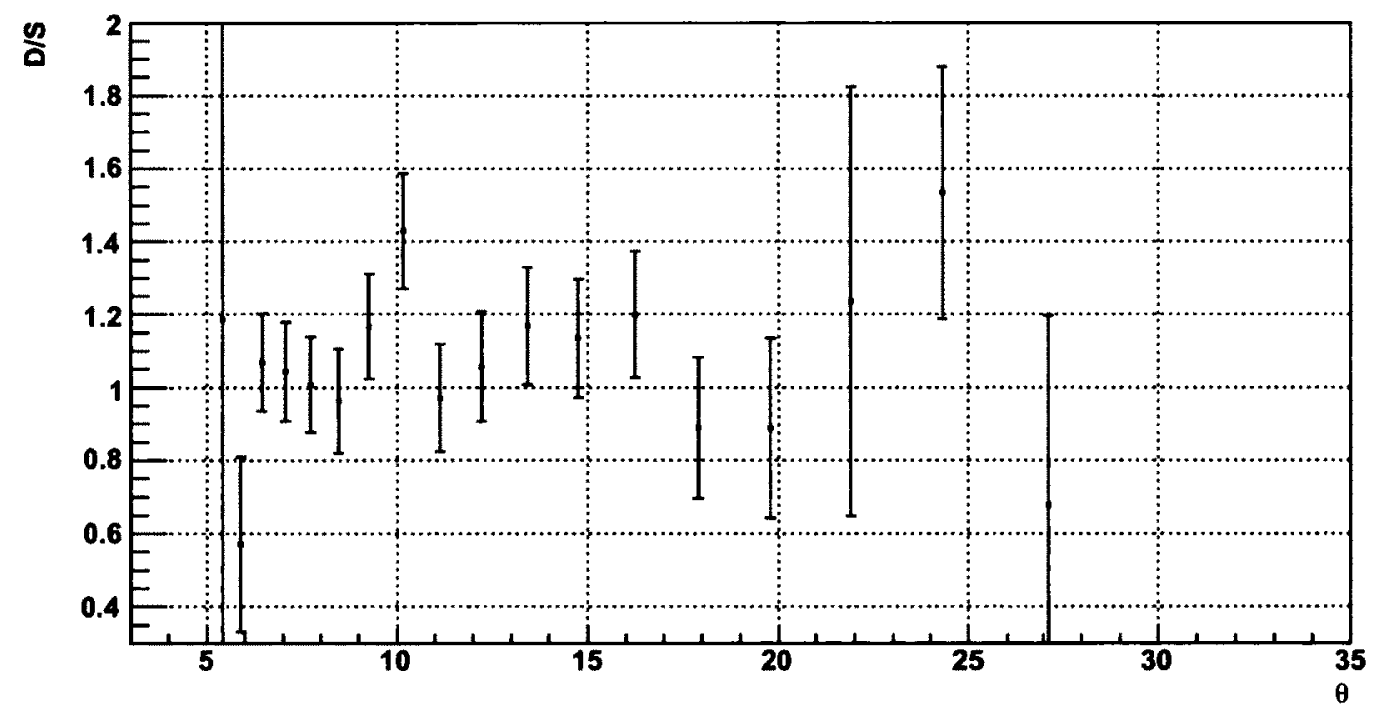

(b) Data/Sim ratio vs $\theta$ in $\Delta$-resonance region of $2.0 \mathrm{GeV}$ data.

FIG. 76. The same data as in Fig. 75, but plotted versus average scattering angle ( $\theta)$. 


\subsection{METHOD TO EXTRACT $g_{1}$ AND $A_{1} F_{1}$}

\subsection{1 'VARIATION" OF THE STANDARD SIMULATION}

The whole chain of steps outlined in the previous sections for the standard simulation is repeated with just one major difference: the model input for the asymmetries $A_{1}$ for both the proton and the neutron are increased by a constant value ${ }^{13}$ of 0.1 . With all other model ingredients being kept constant, this change leads to a change of the spin structure function $g_{1}$ that can be straightforwardly calculated for each kinematic bin within the model:

$$
\delta g_{1}\left(W, Q^{2}\right)=\delta A_{1} \times F_{1} \frac{v^{2}}{v^{2}+Q^{2}}
$$

Correspondingly, the simulated count difference $\Delta n\left(W, Q^{2}\right)$ will change to a new value $\Delta n^{\prime}$. This "non-standard" simulation with $A_{1}=A_{1}$ (standard) +0.1 is performed generating an about equal number of Monte-Carlo events. The final reconstructed data is then multiplied with the same overall scaling factor SF as for the standard simulation and then further (cross-)normalized by one additional factor $S F_{\text {ext }}=\left(\sigma_{1}^{p} / \sigma_{2}^{p}\right) /\left(N_{1} / N_{2}\right)$ to account for the change in cross section map and the (slight) difference in the number of the generated events between the standard and non-standard simulations. Here, $\sigma_{1}^{p}$ and $\sigma_{2}^{p}$ are the total cross sections for the positive $\Delta \sigma$ maps used for the standard and non-standard simulations and, $N_{1}$ and $N_{2}$ are the corresponding numbers of generated events. See Fig. (77) to see how the polarized count differences look (in one particular $Q^{2}$ bin) in experimental and simulated data after such normalizations (for all other $Q^{2}$ bins, see Figs. 71 and 72).

This change of the simulated $\Delta n\left(W, Q^{2}\right)$ to a new value $\Delta n^{\prime}$ can be correlated to the increase in $g_{1}$ by solving for the two parameters $A$ and $B$ of the linear equation,

$$
\Delta n(\operatorname{simul})=A+B \cdot \delta g_{1}
$$

where $A\left(W, Q^{2}\right)$ is the result for the simulated $\Delta n$ for the standard set of model inputs i.e., $A\left(W, Q^{2}\right)=\Delta r^{\text {standard }}\left(W, Q^{2}\right)$, and $B\left(W, Q^{2}\right)$ is the proportionality factor representing the change in $\Delta n(\operatorname{sim})$ per unit change in $g_{1}$, as given by:

$$
B\left(W, Q^{2}\right)=\frac{\Delta n^{\prime}-\Delta n}{\delta g_{1}} .
$$

The proportionality factor $B\left(W, Q^{2}\right)$ is then determined for each of the kinematic bins

\footnotetext{
${ }^{13}$ We arbitrarily chose 0.1 in the inelastic region, but could also have used any other value (not too big, however).
} 


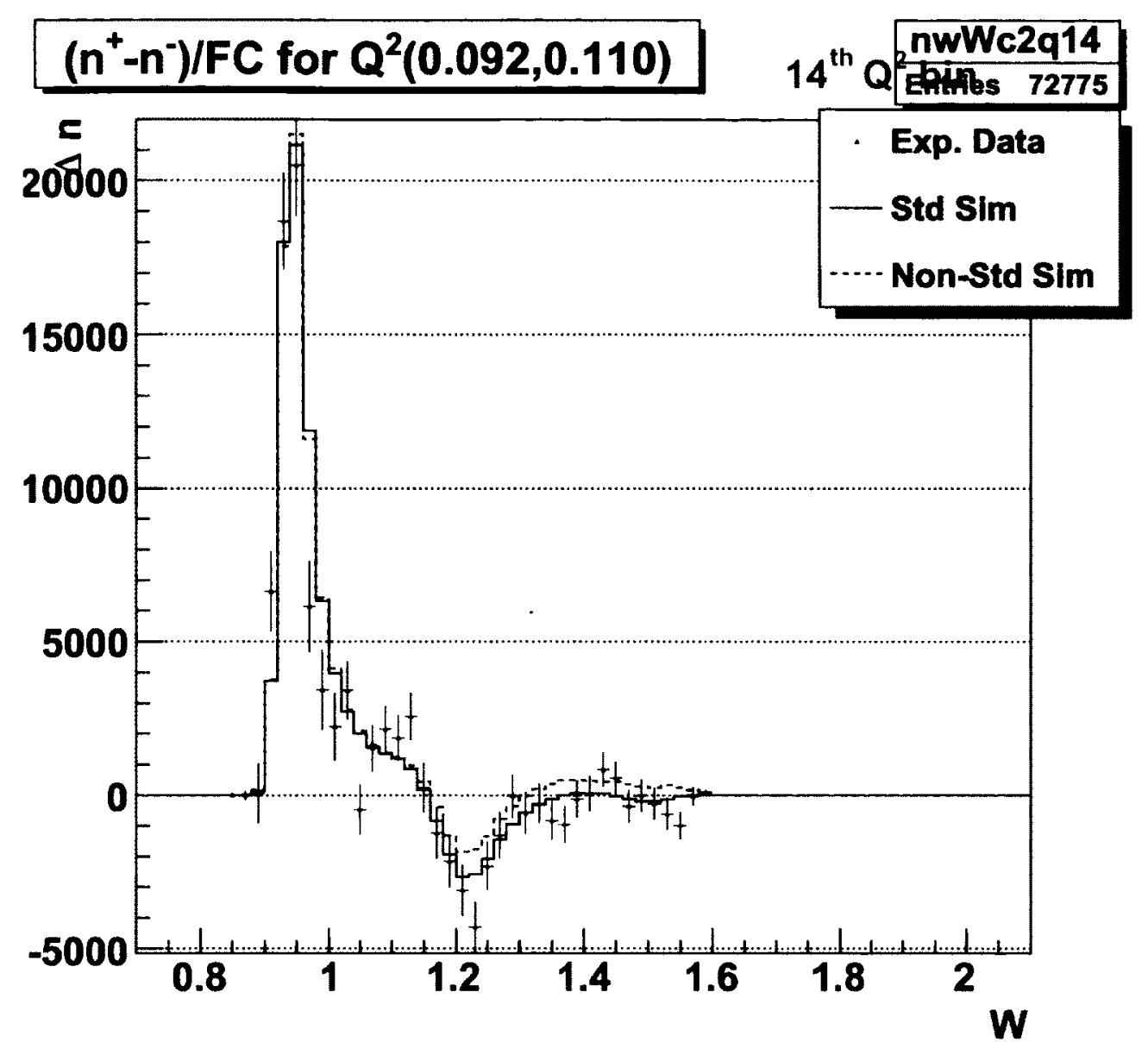

FIG. 77. $\Delta n$ of experimental data and two versions of simulations in one particular $Q^{2}$ bin for $1.3 \mathrm{GeV}$ case (for data on more $Q^{2}$ bins, see Fig. 71).

(in $\left(W, Q^{2}\right)$ ) in which the experimental data has been histogrammed. For this purpose, using the RCSLACPOL program, we produce two values of structure function $g_{1}$ in each kinematic bin - one ( $g_{1}^{\text {Standard }}$ ) corresponding to the standard simulation and the other ( $\left.g_{1}^{\text {non-standard }}\right)$ corresponding to the non-standard simulation. By, dividing the above change in the count difference with the difference $\delta g_{1}$ of the two structure functions, we get the proportionality factor for the bin.

In principle (and ignoring the other enumerated possible sources of disagreement between data and simulation), we can then easily find the "amount of change" $\delta g_{1}$ to be added to the standard model $g_{1}$ to get perfect agreement:

$$
g_{1}^{\text {extr }}\left(W, Q^{2}\right)=g_{1}^{\text {Standard }}+\frac{\Delta n^{\text {data }}\left(W, Q^{2}\right)-\Delta n^{\text {standard }}\left(W, Q^{2}\right)}{B\left(W, Q^{2}\right)}
$$




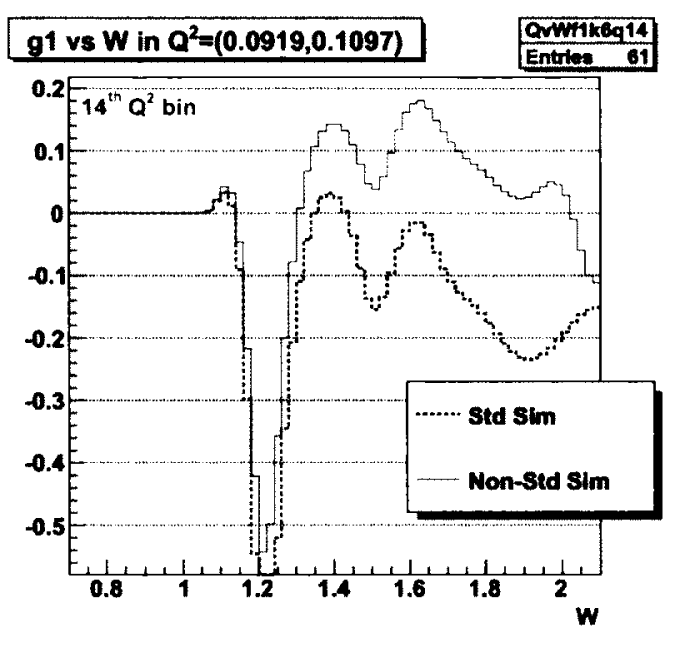

(a) $g_{1}$ for standard and non-standard simulation

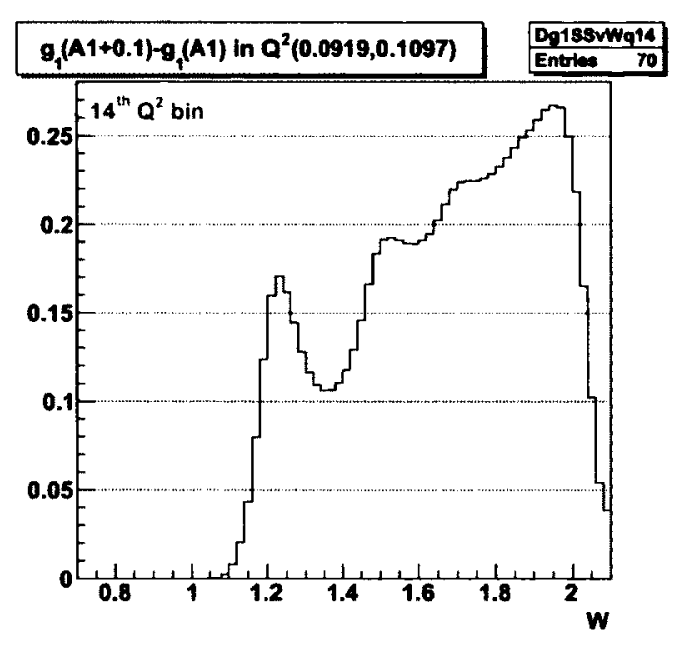

(b) Difference of the two $g_{1}$

FIG. 78. Plots showing the change in model $g_{1}$ due to the change of $A_{1}$ to $A_{1}+0.1$.

where the values of $\Delta n^{\text {data }}$ and $\Delta n^{\text {standard }}$ come from the polarized count differences $\Delta n$ in the experimental data and the standard simulation respectively (as shown, for example, by the red points and black histograms in Fig. 77 for one particular $Q^{2}$ bin).

It is also straightforward to propagate the statistical error to the extracted $g_{1}$. The statistical error in this extracted quantity totally comes from the error in the experimental counts $\Delta n^{\text {data }}$ (assuming there is no error in the model quantities involved and also in the simulation counts because we did our simulation with large enough statistics to warrant ignoring the errors) as follows:

$$
\sigma\left(g_{1}^{e x t r}\left(W, Q^{2}\right)\right)=\frac{\sigma\left(\Delta n^{d a t a}\left(W, Q^{2}\right)\right)}{B\left(W, Q^{2}\right)}
$$

The values of $g_{1}$ and its errors thus extracted from $1.3 \mathrm{GeV}$ data for one $Q^{2}$ bin is shown in Fig. (4.80(b)). Similar results for all the bins from two beam energy data sets in different kinematic bins can be seen in Fig. 90 (next chapter).

Because we are also interested in measuring the forward spin polarizability and the extended GDH integral, we also extract the product $A_{1} F_{1}$ which enter these integrals. We followed the exact same procedure for $g_{1}$ as outlined above. We determined new proportionality factors in each kinematic bin, again using Eq. 140 as before but with the denominator replaced, this time, with the corresponding change in $A_{1} F_{1}$ (instead of the change in $g_{1}$ ). Then we can use the following expression (similar to equation 137) to 

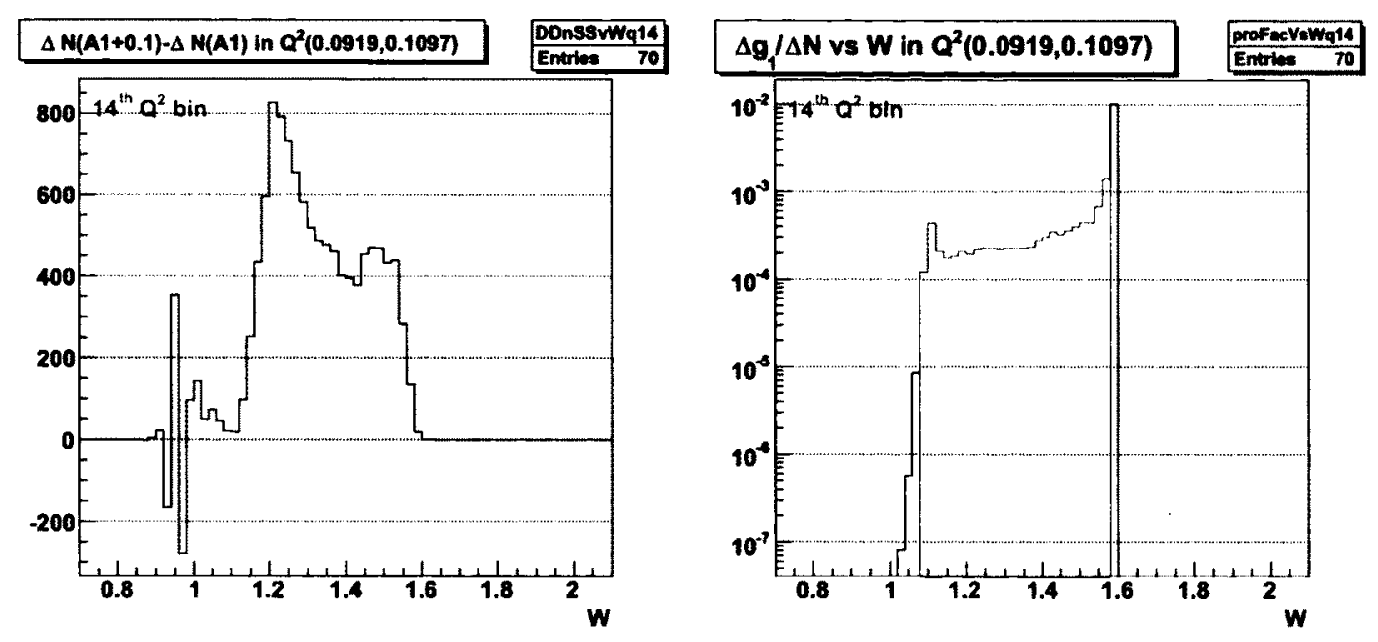

(a) Change in $\Delta n($ sim $)$ simulated count difference (b) Proportionality factor $\left(1 / B\left(W, Q^{2}\right)\right)$ for 1.3 i.e. $\Delta N=\Delta n^{\prime}\left(A_{1}+0.1\right)-\Delta n\left(A_{1}\right)$ due to the change GeV case. Black is the originial values. Red is the of $A_{1}$ to $A_{1}+0.1$ (for $1.3 \mathrm{GeV}$ case). ones kept after discarding the first or last few (low statistics bins) that had unreasonably high (suddenly changing) ratios.

FIG. 79. Plots for $\Delta n(\operatorname{sim})$ and the corresponding proportionality factors.

extract $A_{1} F_{1}\left(W, Q^{2}\right)$ :

$$
A_{1} F_{1}^{\text {extr }}\left(W, Q^{2}\right)=A_{1} F_{1}^{\text {Standard }}+\frac{\Delta n^{\text {data }}\left(W, Q^{2}\right)-\Delta n^{\text {standard }}\left(W, Q^{2}\right)}{B_{A_{1} F_{1}}\left(W, Q^{2}\right)}
$$

where

$$
B_{A_{1} F_{1}}\left(W, Q^{2}\right)=\frac{\Delta n^{\prime}-\Delta n}{\delta A_{1} F_{1}}
$$

And, the errors on $A_{1} F_{1}$ can also be dealt in the same way as on $g_{1}$. 


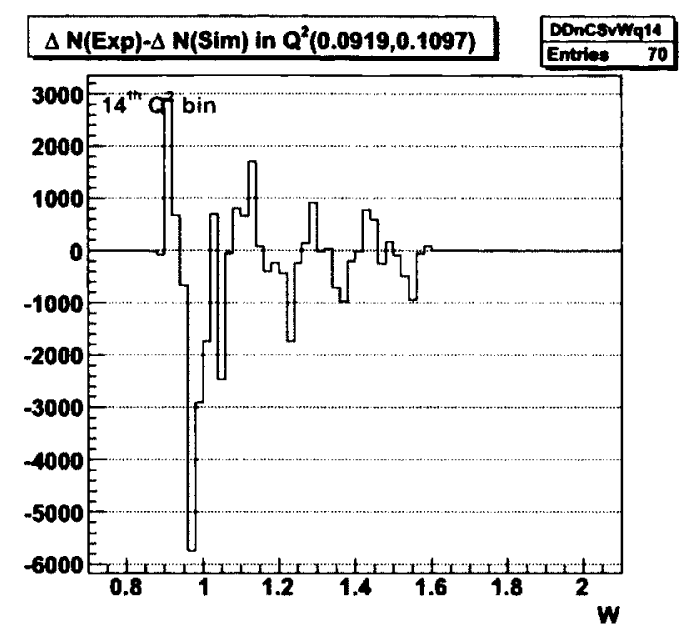

(a) Difference $\Delta n($ data $)-\Delta n(\operatorname{sim})$ (for $1.3 \mathrm{GeV}$ case).

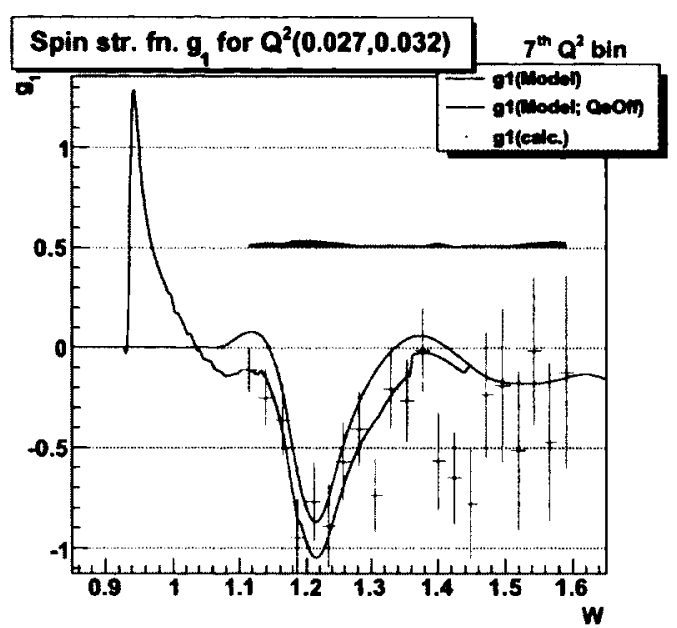

(b) Caculated $g_{1}$ from $1.3 \mathrm{GeV}$ data.

FIG. 80. Plots for $\Delta n$ and the corresponding extracted $g_{1}$.

\subsection{SYSTEMATIC ERROR ESTIMATIONS IN $g_{1}$ AND $A_{1} F_{1}$}

The following systematic error contributions on the final extracted $g_{1}\left(W, Q^{2}\right)$ can be separated into two categories [93]:

1. Overall scale errors (see Sec. 4.16.1). These are errors that affect the proportionality constant $B$ defined in Sec. 4.15.1 equally in all bins and are mostly due to uncertainties in $P_{b} P_{t}$ and target thickness. The total scale uncertainty should be evaluated separate from the remaining systematic errors and quoted as a percent error in the final presentation of the data.

2. Point-to-point uncorrelated errors. These are mostly additive errors, although there may be some kinematic-dependent uncertainty in quantities like the $\mathrm{CC}, \mathrm{EC}$ and tracking efficiency. These errors are evaluated in sequence, as additional uncertainties on $g_{1}$ bin by bin, and added in quadrature to get the overall uncertainty. For integrals over $g_{1}$, these errors are added incoherently (in quadrature) with the appropriate weights; e.g., for $\Gamma_{3}\left(Q^{2}\right)=\Sigma\left(g_{1}\left(x, Q^{2}\right) x^{2} \Delta x\right)$ the corresponding systematic error would be $\delta \Gamma_{3}\left(Q^{2}\right)=\left[\Sigma\left(\delta g_{1}\left(x, Q^{2}\right) x^{2} \Delta x\right)^{2}\right]^{1 / 2}$.

3. Model errors which vary point to point but are correlated. 


\subsubsection{CROSS NORMALIZATION SIMULATION / DATA}

Since the normalization factor $S F$ comes from a comparison of data and simulation in the quasi-elastic region $0.9 \leq W \leq 1.0$, one has to evaluate all systematic effects that can influence either one of these ingredients. The first and most important systematic error due to this factor is simply the statistical error described at the end of Sec. 4.13. The remaining systematic errors are listed below and have to be added in quadrature to the statistical one. One should check if the overall $\chi^{2}$ for the comparison over all $Q^{2}$ bins is compatible with the combined statistical and systematic error on $S F$; otherwise, it may be necessary to increase the total error accordingly.

On the simulation side, one has to account for the following:

1. The calculated cross section difference depends most sensitively on the beam energy and the scattering angle. Since radiative effects are a second order effect here, it suffices to calculate the theoretical Born cross section differences for both the standard beam energy and average angle for each $Q^{2}$ bin, and then repeat the calculation for i) a beam energy increased by $5 \mathrm{MeV}$ and ii) a decrease of the scattered electron energy by $5 \mathrm{MeV}$ and iii) a scattering angle increased by $1 \mathrm{mrad}$. The relative change in $\delta \sigma$ contributes to the total error for each bin.

2. To a lesser extent, the model input for the form factors $G_{E}, G_{M}$ for both proton and neutron also contribute to the simulated uncertainty. Therefore, the model Born asymmetry also has to be recalculated with the following changes: i) use the simple dipole fit for $G_{M}^{p}$ ii) use the dipole fit for $G_{M}^{n}$ iii) use the dipole fit for $G_{E}^{p}$ and iv) use $G_{E}^{n}=0$.

3. Vary the scale factor within the uncertainty for the kinematics-dependent part of the $\mathrm{CC}, \mathrm{EC}$ and tracking efficiencies in the quasi-elastic region (an overall trigger and tracking efficiency will drop out).

4. The main effect of radiation is to decrease the measured cross section difference in the quasi-elastic region ("out"-radiation). Any discrepancy between simulated and "true" depletion due to that effect yields a systematic error on the scale factor. By looking at various models of radiative effects (e.g., a simple-minded "equivalent radiator" model vs. the full-blown RCSLACPOL code) one can quantify this uncertainty. However, we did not do this in the end. Instead, we just assumed an overall 
error consistent with the observed fluctuation by $Q^{2}$ bin of $10 \%$ on the overall scale factor.

For the data, one has to vary the possible background contributions within their systematic uncertainties: Contributions to $\delta \boldsymbol{n}$ in the quasi-elastic region due to possible contributions from bound and free polarized protons (contributions from bound deuterons are minimal and cancel mostly), and contributions from mis-identified pions and pair symmetric electrons.

In the present analysis, we considered ten distinct contributions to the systematic error in the measured $g_{1}$ (and similarly to $A_{1} F_{1}$ ) as follows:

1. Possible Error in the overall scaling factor

2. Effect due to the contaminations of polarized $\mathrm{H}$ in the target and $\pi^{-}$in the scattered electrons.

3. Possible error in the beam energy measurement

4. Possible error in the CC-inefficiency estimation

5. Effect due to the $e^{+} e^{-}$pair symmetric contamination

6. Possible error in the estimation of radiation lengths (especially RADA)

7. Model variation using preliminary version (v1) of $A_{1}$ model by Guler/Kuhn (2008-9)

8. Model variation using old version of $A_{2}$ resonance model

9. Model variation of $F_{2}$ (and proportionally of $F_{1}$ )

10. Model variation of $\mathrm{R}\left(F_{2}\right.$ changed)

For the ease of description later on, these ten components will be referred to by the index " $k$ " with its value indicating the position in the list. So, the error due to scaling factor will be identified with $\mathrm{k}=1$ and so on.

Possible Error due to the overall scaling factor This error is due to the uncertainties in the overall scaling factor (SF) (see Sec. 4.16.1). This contribution is estimated by assuming that the uncertainties in SF is not more than $10 \%$. Thus considering the worst case scenario of $10 \%$ error in SF, we estimate the corresponding error in $g_{1}$ as follows:

$$
\Delta g_{1}^{S F}\left(W, Q^{2}\right)=g_{1}^{s t d}\left(W, Q^{2}\right)+\frac{\Delta n^{\text {data }}\left(W, Q^{2}\right)-1.1 \cdot \Delta n^{s t d}\left(W, Q^{2}\right)}{1.1 \cdot B\left(W, Q^{2}\right)}-g_{1}^{\text {data }}\left(W, Q^{2}\right)
$$


with "std" shorthand used for "standard" model or the corresponding simulation.

Error from Polarized $\mathrm{H}$ in target and $\pi^{-}$contaminations This contribution from polarized $\mathrm{H}$ in target and $\pi^{-}$contamination is evaluated as follows,

$$
\Delta g_{1}^{\text {cont }}\left(W, Q^{2}\right)=g_{1}^{s t d}\left(W, Q^{2}\right)+\frac{\Delta n^{\text {data }}\left(W, Q^{2}\right) \cdot 1.025-\Delta n^{s t d}\left(W, Q^{2}\right)}{B\left(W, Q^{2}\right)}-g_{1}^{\text {data }}\left(W, Q^{2}\right)
$$

where we assume that the contamination is not more than $2.5 \%$.

Possible error in the beam energy measurement This contribution is evaluated assuming the error in beam energy measurement is not more than $10 \mathrm{MeV}$, so the either the experimental data or the standard-simulation data can be analyzed assuming the beam energy was different by $10 \mathrm{MeV}$. In this analysis, the the energy was increased by $10 \mathrm{MeV}$ in the simulated data.

$$
\Delta g_{1}^{E b}\left(W, Q^{2}\right)=g_{1}^{s t d}\left(W, Q^{2}\right)+\frac{\Delta n^{d a t a}\left(W, Q^{2}\right)-\Delta n_{E b+}^{s t d}\left(W, Q^{2}\right)}{B\left(W, Q^{2}\right)}-g_{1}^{d a t a}\left(W, Q^{2}\right)
$$

where $\Delta r_{E b+}^{s t d}$ is now the simulated $\Delta r^{s t d}$ obtained by analyzing the data from the standard simulation as usual but with a beam energy that was $10 \mathrm{MeV}$ more than the standard value.

Possible error in the CC-inefficiency estimation This contribution is estimated by assuming a maximum of $50 \%$ error in the estimated inefficiency as follows:

$$
\Delta g_{1}^{E b}\left(W, Q^{2}\right)=g_{1}^{s t d}\left(W, Q^{2}\right)+\frac{\Delta n^{\text {data }}\left(W, Q^{2}\right)-\Delta n_{0.5 C C i}^{\text {std }}\left(W, Q^{2}\right)}{B\left(W, Q^{2}\right)}-g_{1}^{\text {data }}\left(W, Q^{2}\right)
$$

where $\Delta n_{0.5 C C i}^{s t d}$ is now the simulated $\Delta n^{s t d}$ obtained after applying $50 \%$ more inefficiency instead of the actually estimated value.

Possible error due to $e^{+} e^{-}$pair symmetric contamination The contribution due to $e^{+} e^{-}$pair symmetric contamination is calculated as follows:

$$
\Delta g_{1}^{E b}\left(W, Q^{2}\right)=g_{1}^{s t d}+\frac{\Delta n^{d a t a} \cdot \frac{1}{1+f\left(e^{+} e^{-}\right)}-\Delta n^{s t d}}{B\left(W, Q^{2}\right)}-g_{1}^{d a t a}\left(W, Q^{2}\right)
$$

where $\mathrm{f}\left(e^{+} e^{-}\right)$is the $e^{+} e^{-}$fraction from the EGlb fit by N. Guler [35] (used the closest available energies). 
Radiative correction uncertainty Here, we need to change the parameter that most influences radiative corrections, the number of radiation lengths before (RADB) and after (RADA) the scattering. By increasing both numbers by $10 \%$, we should have a safe upper limit on practically all uncertainties coming from the radiative procedure itself. But, to simplify the situation, we increased the RADA parameter in RCSLACPOL by $20 \%$ and repeated the full-statistic simulation. As a result the simulated count difference in each kinematic bin changed from $\Delta n^{\text {standard }}$ to a new value $\Delta n^{\text {rad }}$. This change can be converted to the corresponding inferred change in $g_{1}$ by using the same proportionality factors $B\left(W, Q^{2}\right)$ as used earlier in the $g_{1}$ (or $\left.A_{1} F_{1}\right)$ extraction/calculation. In other words, for a given kinematic bin this particular contribution to the systematic error is calculated as:

$$
\Delta g_{1}^{r a d}\left(W, Q^{2}\right)=g_{1}^{s t d}+\frac{\Delta n^{\text {data }}\left(W, Q^{2}\right)-\Delta n^{\text {standard }}\left(W, Q^{2}\right)}{B\left(W, Q^{2}\right)}-g_{1}^{\text {data }}\left(W, Q^{2}\right)
$$

where the proportionality factor $B\left(W, Q^{2}\right)$ for the bin is exactly the same as that used to calculate $g_{1}$ earlier.

\subsubsection{MODEL UNCERTAINTIES}

The remaining four components in the total systematic uncertainty (the last four in the list 4.16.1) account for the model uncertainty contributions. For this purpose, we changed the values of two of the model parameters "AsymChoice" and "SFchoice" (each takes value of 11 , in the standard case)

We repeated the full statistics simulation four more times by changing the values of two RCSLACPOL parameters "AsymChoice" and "SFchoice" (which controls the values of model asymmetries and the structure functions, with each taking a value of 11 in the standard case) one by one corresponding to the following four model variations:

1. Variation-1: AsymChoice $=12, \mathrm{SF}$ choic $=11$

2. Variation-2: AsymChoice $=15, \mathrm{SF}$ choic $=11$

3. Variation-3: AsymChoice $=11, \mathrm{SF}$ choic $=12$

4. Variation-4: AsymChoice $=11, \mathrm{SF}$ choic $=13$

where, the different values of the two RCSLACPOL parameters correspond to the following model choices:

1. AsymChoice values are used to determine specific $A_{1} / A_{2}$ models used in the RCSLACPOL program 
(a) 11: Standard Resonance Model 2008-9 Guler/Kuhn (Used for standard simulation)

(b) 12: Variation of $A_{1}$ model

(c) 15: Variation of $A_{2}$ resonance model: Vary the virtual photon asymmetry $A_{2}$ in the resonance region within its fit errors.

2. SFchoice values are used to determine specific $F_{1} / F_{2}$ models.

(a) 11: 2009 version of $F_{1}^{n} / F_{1}^{p} / F_{1}^{d}$ by Peter Bosted/Eric Christie 2009, HERMES (Used for standard simulation) (with $\mathrm{d}$ in $F_{1}^{d}$ denoting a deuteron).

(b) 12 : Same version as 11 , but with fit errors added to $F_{2}$ (and proportionally $F_{1}$ )

(c) 13: Same version as 11 , but with fit errors subtracted from $\mathrm{R}$ ( $F_{2}$ unchanged)

After the simulation data for the above four cases were available, four more data tables (TM1,TM2,TM3 and TM4) were produced for the corresponding model values of $g_{1}, A_{1}$, $F_{1}$ etc. Then, the contributions to the systematic error from each of these four cases of model variation were given as follows:

$$
\Delta g_{1}^{i}\left(W, Q^{2}\right)=g_{1}^{\text {standard }}\left(W, Q^{2}\right)-g_{1}^{i}\left(W, Q^{2}\right)+\frac{\Delta n^{i}\left(W, Q^{2}\right)-\Delta n^{\text {standard }}\left(W, Q^{2}\right)}{B\left(W, Q^{2}\right)}
$$

with " $\mathrm{i}$ " indicating any of the four cases of model variation, $g_{1}^{i}$ being the model prediction for the $i^{t h}$ case as obtained from the corresponding data table "TMi" and the proportionality factor $B\left(W, Q^{2}\right)$ again being exactly the same as used to calculate $g_{1}$ as earlier.

Figs. (82 and 83) show, for example, the different components of the systematic errors (along with the grand total) on $g_{1}$ (from $1.3 \mathrm{GeV}$ data) evaluated in the manner just outlined. Likewise, Figs. ( 84 and 85 ) show similar plots for the $2.0 \mathrm{GeV}$ data.

These ten different components of systematic errors on $g_{1}$ (and similarly on $A_{1} F_{1}$ ) thus calculated separately for both beam energies are later combined as follows:

\subsubsection{COMBINING DATA FROM THE TWO BEAM ENERGIES}

Once the data $g_{1}$ and $A_{1} F_{1}$ and their corresponding errors are evaluated from each beam energy data set, they are combined as follows [63] (to make the description simple, only procedure is described only for $g_{1}$, but, in the end, the exact same procedure is followed for $A_{1} F_{1}$ as well): 
1. First a table is made, separately for each beam energy, of all $\left(Q^{2}, W\right)$ bins with with calculated values of $g_{1}$, their statistical errors and each of the ten components of the systematic errors (making sure to keep the correct signs of the systematic changes) in separate columns (one row is for one bin in $\left(Q^{2}, W\right)$.

2. Then another table is made for the combined values of $g_{1}$, which are evaluated as follows:

(a) If for a given $\left(W, Q^{2}\right)$ bin, $g_{1}$ comes only from one beam energy, then all the entries from that energy go into the "combined" table

(b) If $g_{1}$ has measurements from both beam energies, we combine them with statistical weights as follows:

$$
\begin{array}{rlr}
\text { Sum } 1 & =\sum_{i} \frac{g_{1}(i)}{\left(\Delta g_{1}\right)^{2}(i)} & \text { Sum } 2=\sum_{i} \frac{1}{\left(\Delta g_{1}\right)^{2}(i)} \\
g_{1}(\text { combined }) & =\text { Sum } 1 / \text { Sum } 2 & \sigma g_{1}(\text { combined })=\sqrt{1 / \text { Sum } 2}(1
\end{array}
$$

where the index ' $\mathrm{i}$ ' represents two beam energy $(1.3$ and $2.0 \mathrm{GeV})$ data sets. and $\Delta g_{1}$ indicates the statistical error in $g_{1}$.

3. In principle, each of the individual contributions to the systematic error can also be combined using the same equations. However, we must be careful to distinguish between correlated and uncorrelated errors.

(a) The variations due to scale factor $(\mathrm{k}=1)$, beam energy ( $\mathrm{k}=3)$ and CC-efficiency $(k=4)$ are all un-correlated and, therefore, added in quadrature as follows:

$$
\delta g_{1}(\mathrm{k}=8,10,11, \text { combined })=\sqrt{\left(\sum_{i} \frac{\left(\delta g_{1}\right)^{2}(i)}{\left(\Delta g_{1}\right)^{2}(i)}\right) / \text { Sum } 2}
$$

where, $\delta$ represents the $k^{\text {th }}$ component of the systematic error, whereas, 'Sum2', ' $i$ ' and $\Delta$ have the same meanings as before.

(b) while all other variations are correlated between the two beam energies and should be averaged linearly (WITH sign):

$$
\delta g_{1}(\text { other } \mathrm{k}, \text { combined })=\left(\sum_{i} \frac{\left(\delta g_{1}\right)(i)}{\left(\Delta g_{1}\right)^{2}(i)}\right) / \text { Sum } 2
$$


4. Once each of the $k^{\text {th }}$ component of the systematic errors are combined between the two beam energies, we then proceed to combine them all to get a grand total. This is done by simply adding the ten combined systematic errors in quadrature and taking the square-root of the sum.

The figures 86 and 88 show the breakdown of the total contribution to the systematic error from different sources. We can see that the dominant contribution comes from the uncertainties in the overall scale factor (the cyan band indicated with SF-err in the legend) which is used to normalize the simulated data to make them comparable with data. This uncertainty comes mainly from those in $P_{b} P_{t}$ and target size measurements. Next big contributions seem to come from the model and radiative corrections. Near the $\Delta$-resonance region, the effect of beam energy uncertainty also seems to be very pronounced. The breakdown of the different components (but combined between the two beam energies) of the total systematic errors are also shown separately in the figures 86 and 88 


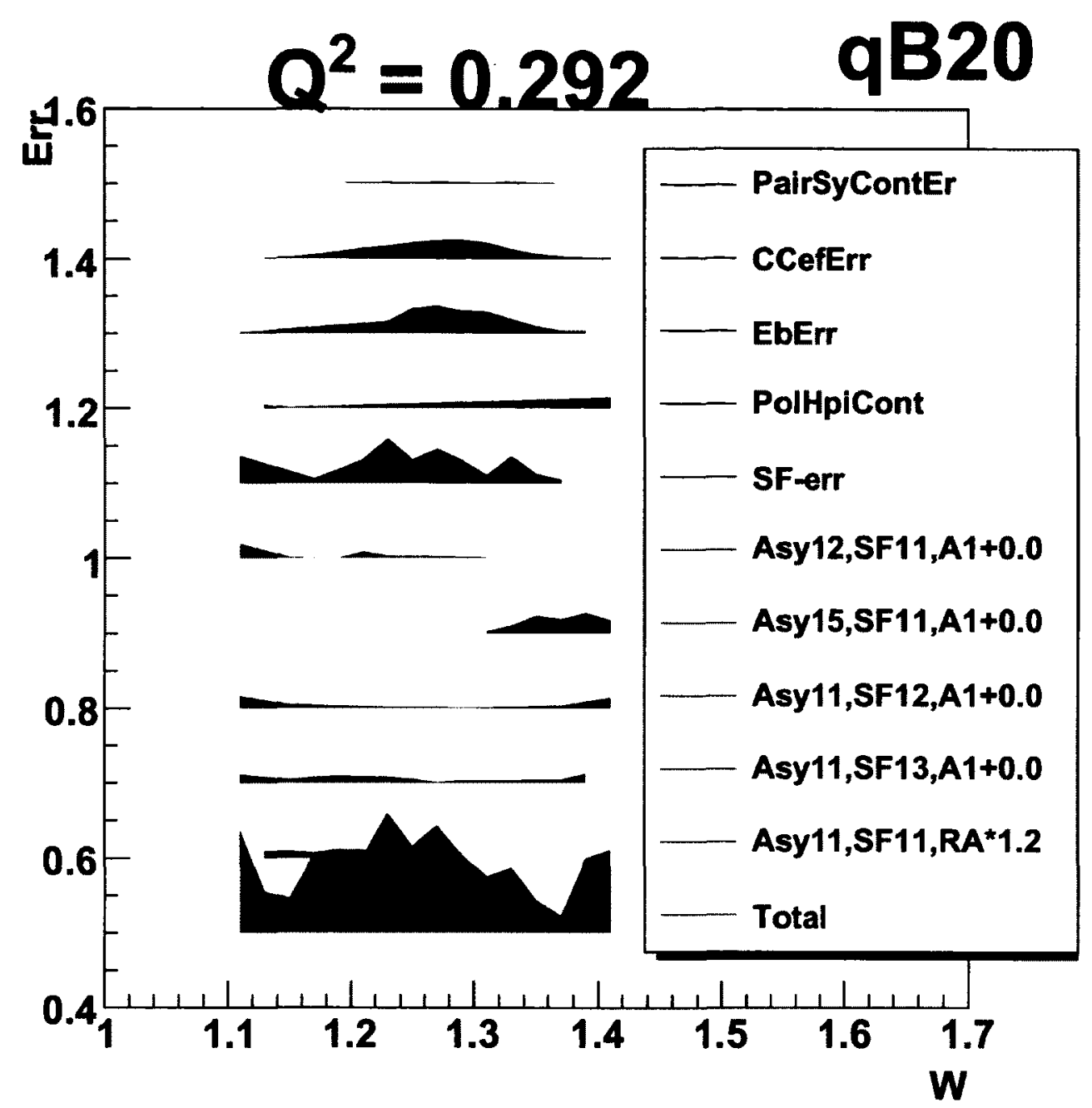

FIG. 81. Various components of systematic error (see Secs. 4.16 and 4.16.1) on $g_{1}$ plotted against $W$ in a $Q^{2}$ bin (1.3 GeV data). The band width represents the size of the errors. The vertical position of each band has no physical meaning (arbitrarily chosen for the convenience of display). The first five (blue) bands are the contributions due to $e^{+} e^{-}$contamination (see Sec. 4.16.1), CC-inefficiency (see Sec. 4.16.1), errors in beam energy measurement (see Sec. 4.16.1), polarized background $\left(H, \pi^{-}\right.$etc - see Sec. 4.16.1) and scaling factor uncertainties (see Sec. 4.16.1) respectively. The first (top) magenta band is the contribution due to the uncertainties in the radiative corrections (see Sec. 4.16.1), next four (magenta) are due to model uncertainties (see Sec. 4.16.2) and the last (green) one is the total error after properly combining all components. For similar plots in other $Q^{2}$ bins see Figs. 82 and 83. 

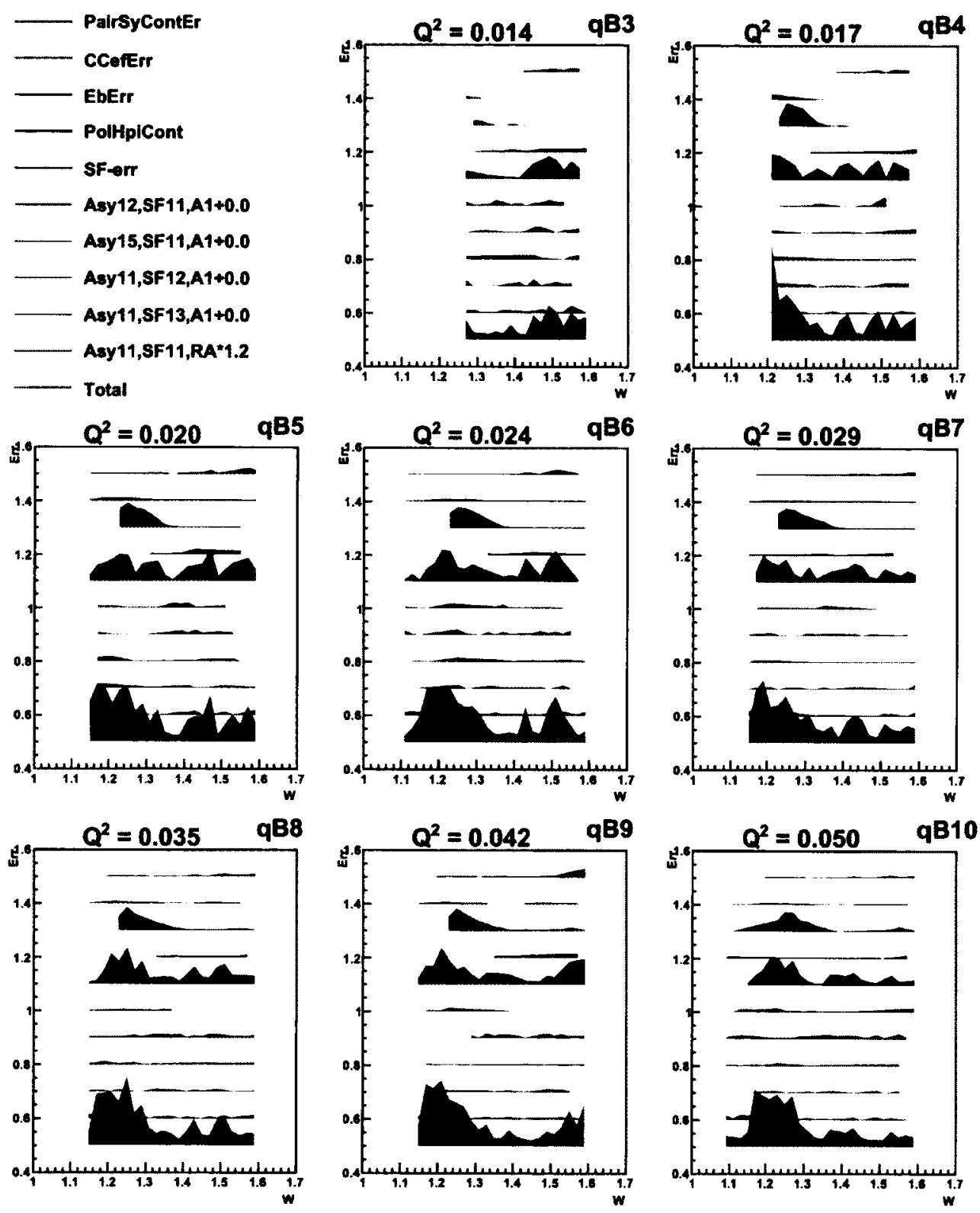

FIG. 82. Plots like that shown in Fig. 81 showing various components of systematic error on $g_{1}$ plotted against $W$ in different $Q^{2}$ bins for $1.3 \mathrm{GeV}$ data. 


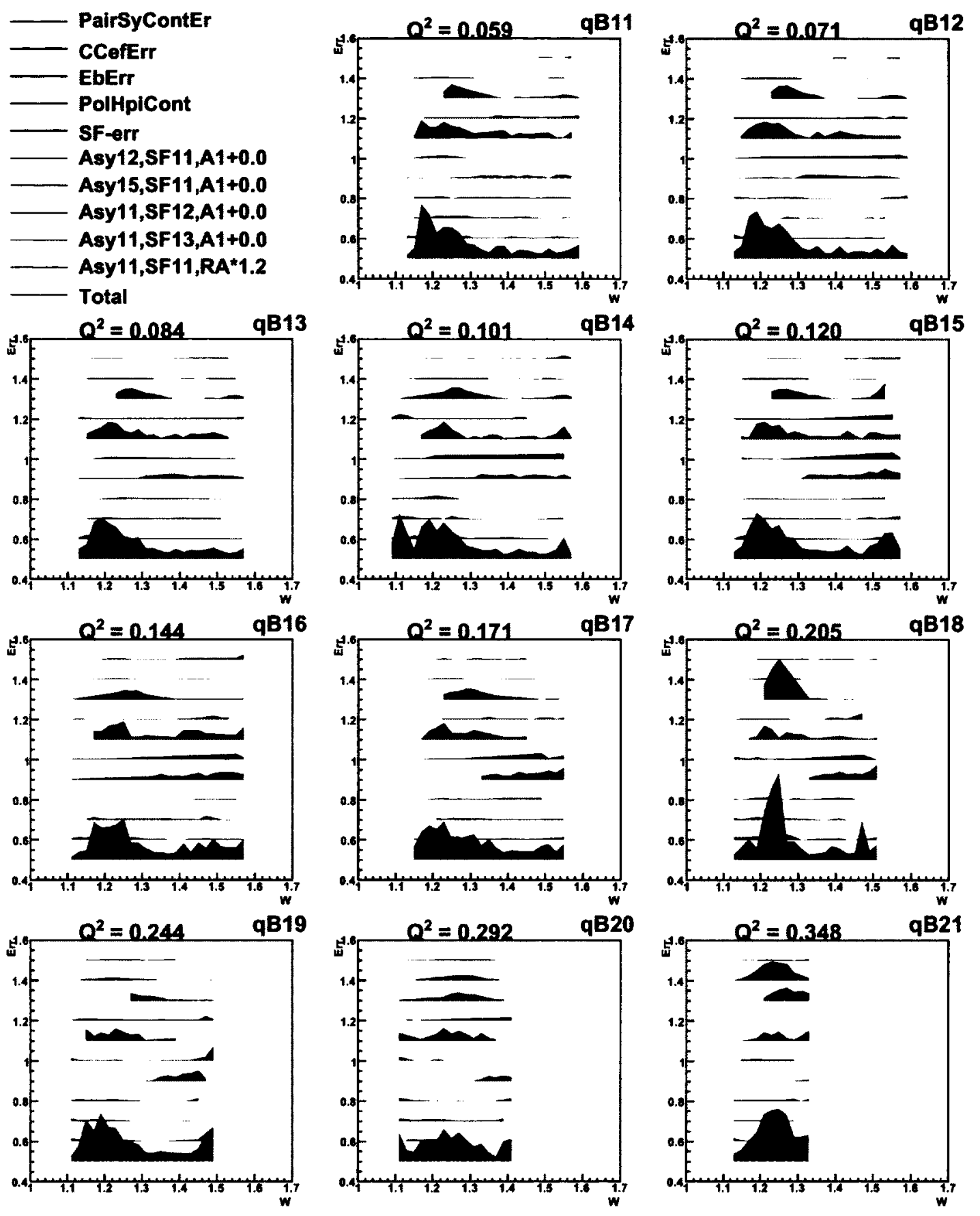

FIG. 83. Systematic error components in remaining $Q^{2}$ bins (continuation of Fig. 82. 

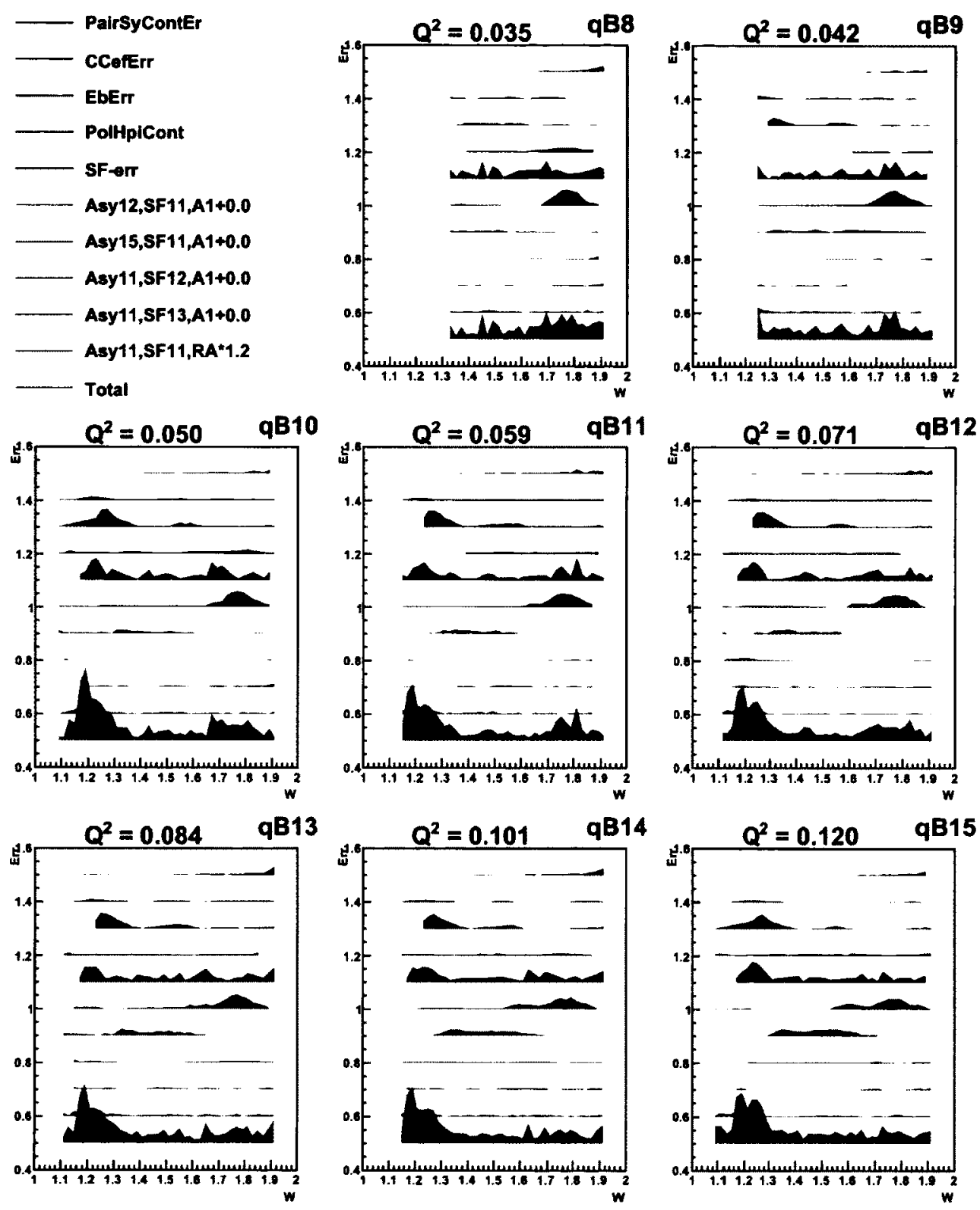

FIG. 84. Plots similar to those shown in Fig. 82 but for $2.0 \mathrm{GeV}$, showing various components of systematic error on $g_{1}$ plotted against $W$ in different $Q^{2}$ bins. 

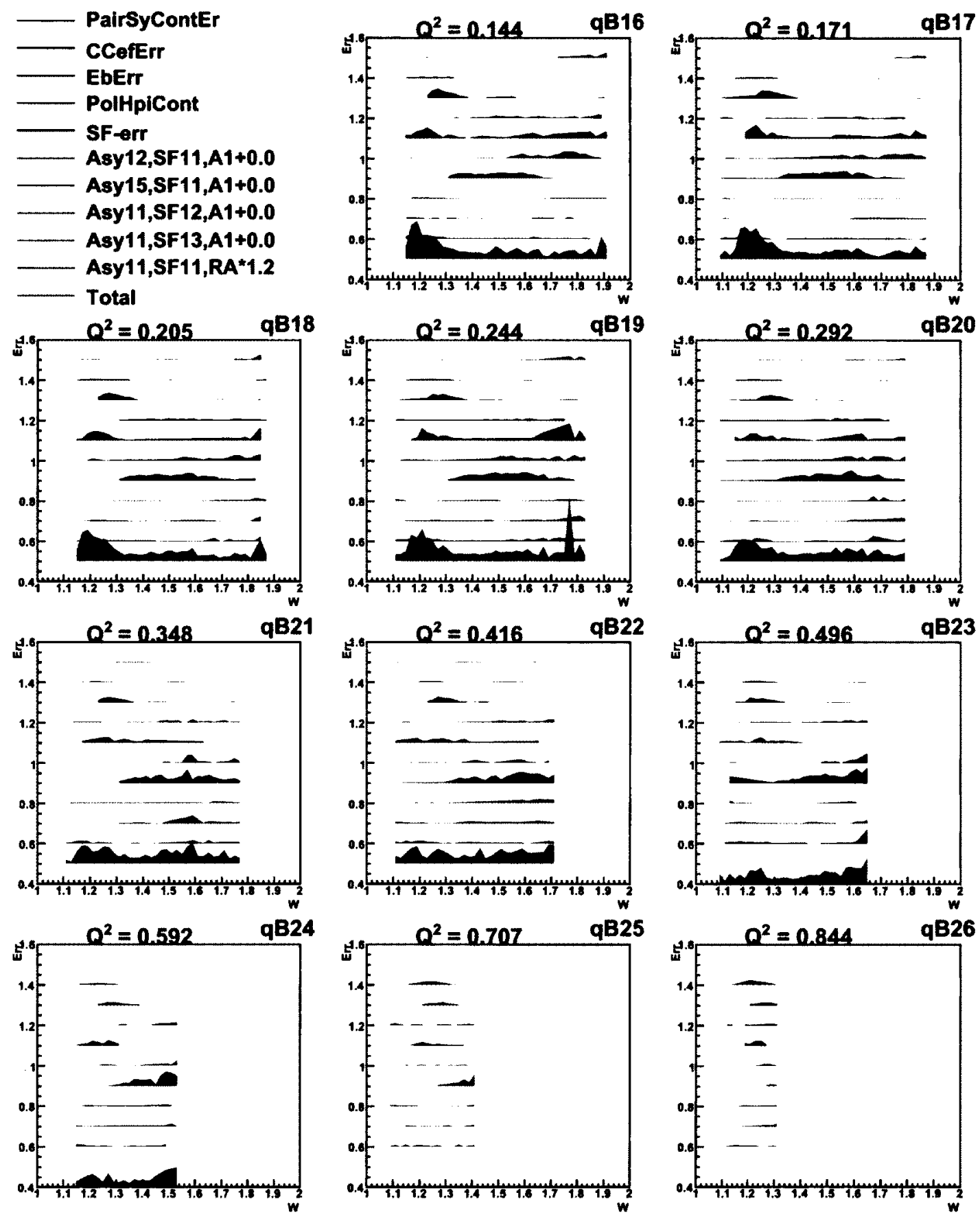

FIG. 85. Systematic error components in remaining $Q^{2}$ bins (continuation of Fig. 84 . 

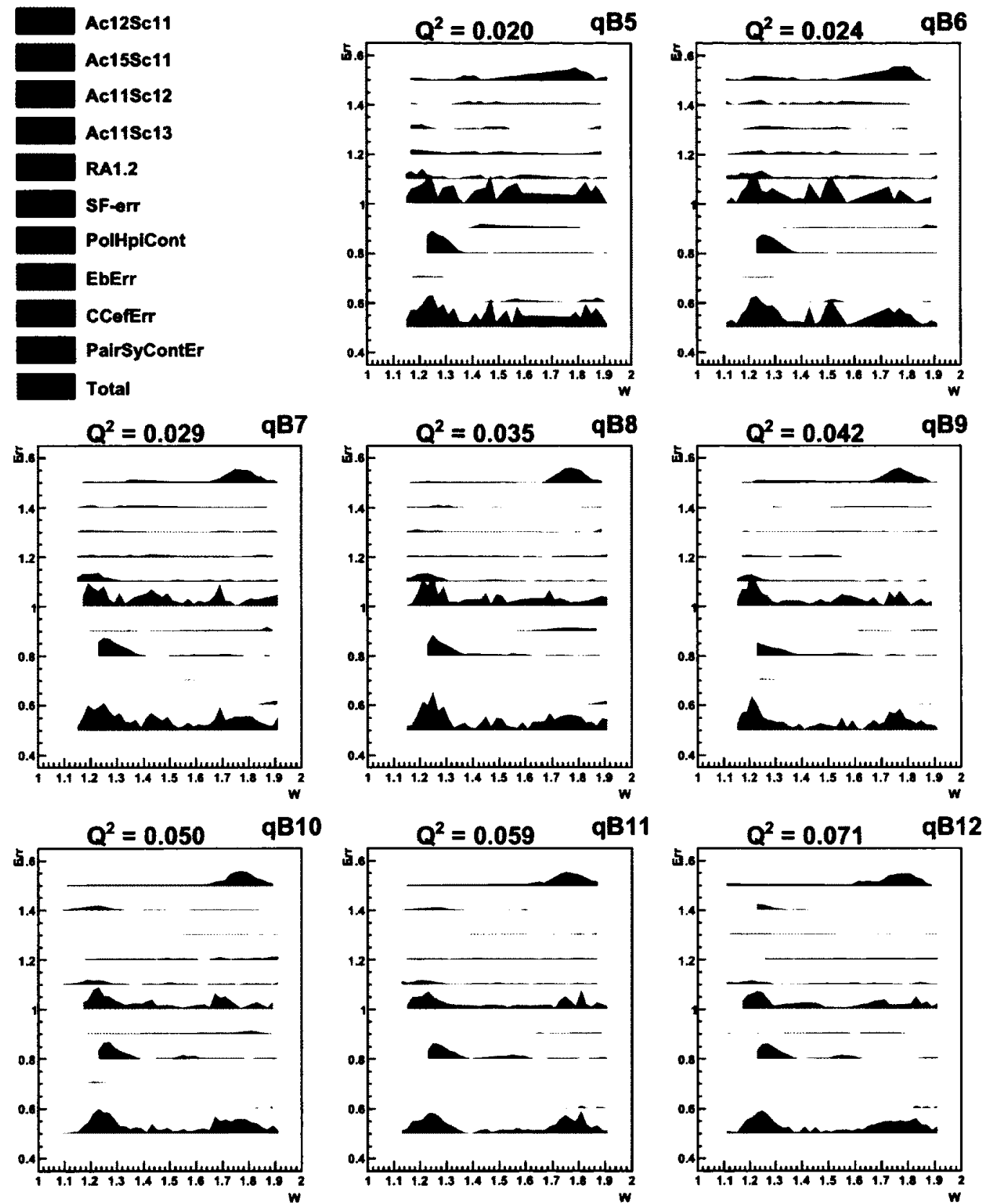

FIG. 86. Breakdown of systematic errors in $g_{1}$ (after combining data from the two energy data sets) in the first few $Q^{2}$ bins. See Fig. 81 for the description of the different systematic error components. 

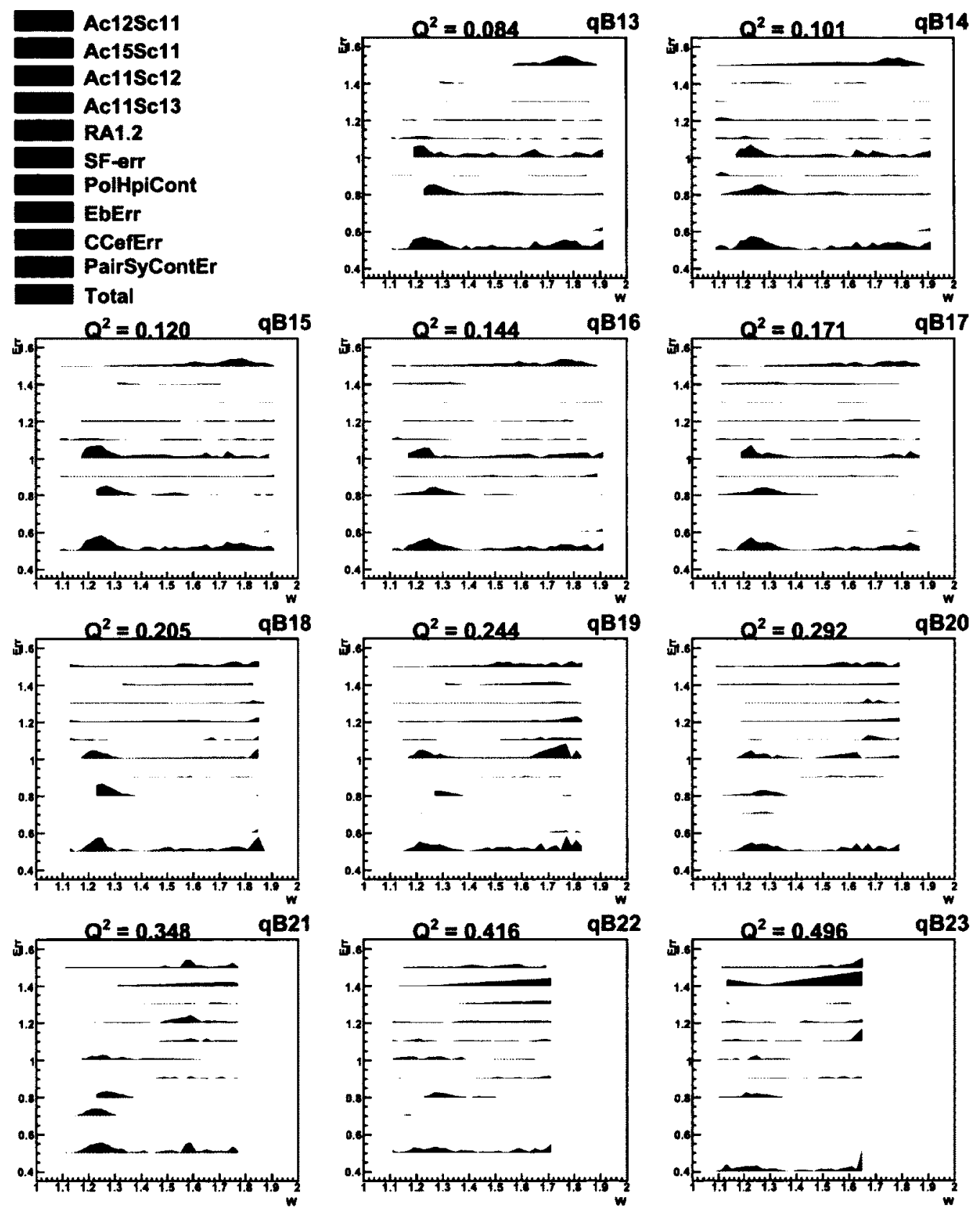

FIG. 87. Plots as in Fig. 86 but in the remaining higher $Q^{2}$ bins. 

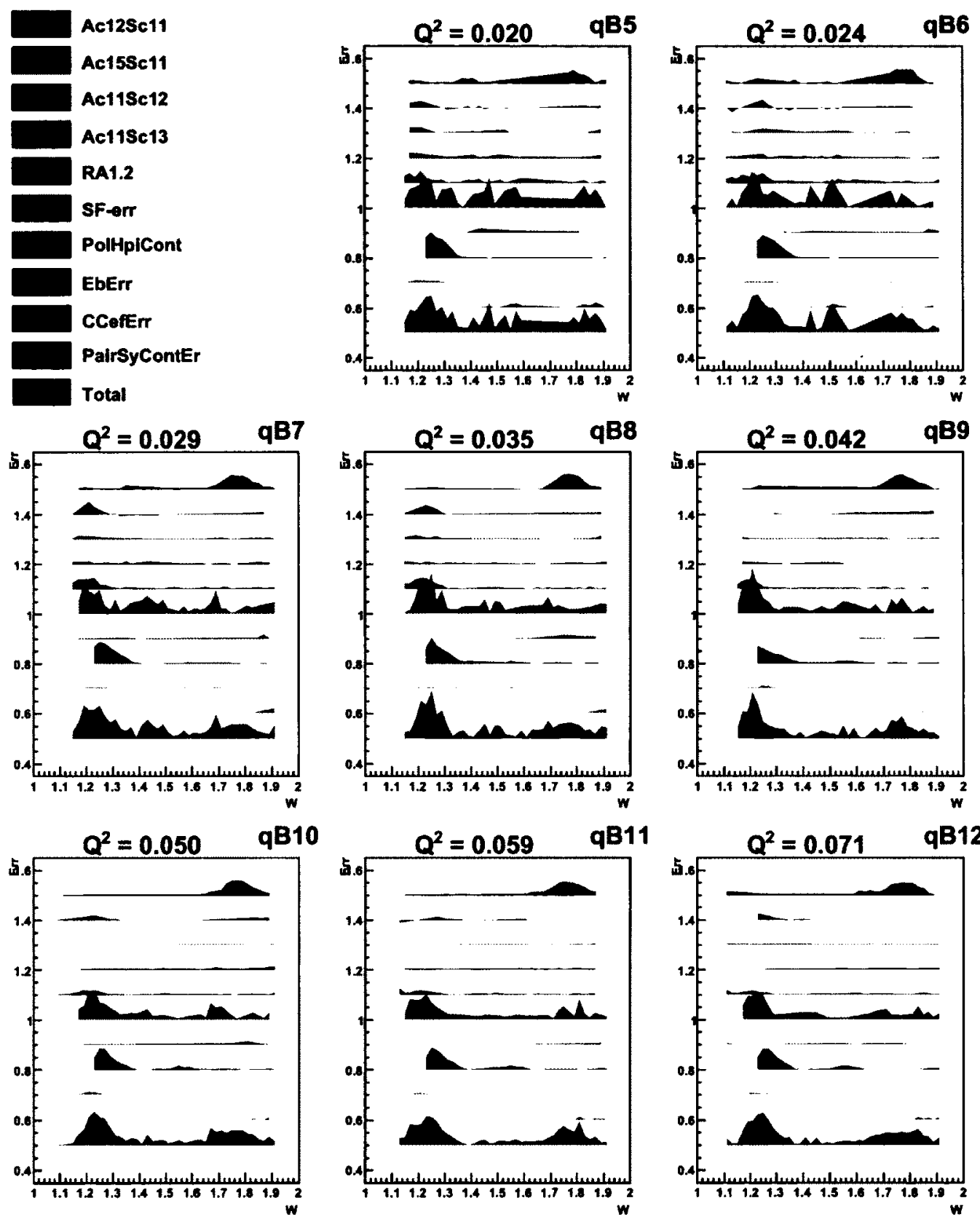

FIG. 88. Breakdown of systematic errors in $A_{1} F_{1}$ (after combining data from the two energy data sets) in the first few $Q^{2}$ bins. See Fig. 81 for the description of the different systematic error components. 


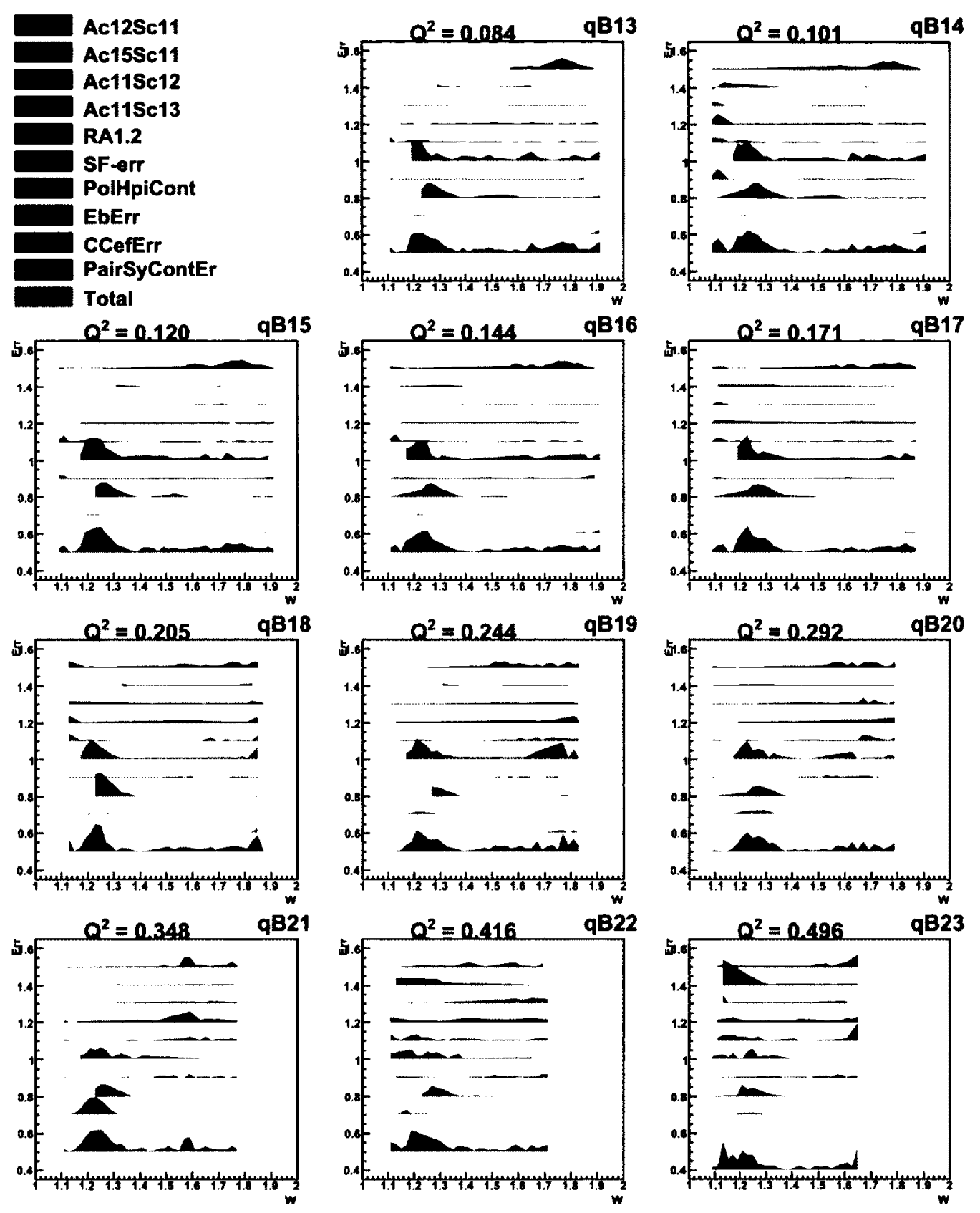

FIG. 89. Plots as in Fig. 88 but in the remaining higher $Q^{2}$ bins. 


\section{CHAPTER 5}

\section{RESULTS}

\subsection{THE EXTRACTED VALUES OF $g_{1}$ AND $A_{1} F_{1}$}

With the methods outlined in the previous chapter, $g_{1}, A_{1} F_{1}$, and and their uncertainties were determined from the EG4 deuteron target data. These data were divided into $21 Q^{2}$ bins (between about 0.02 and $0.7 \mathrm{GeV}^{2}$ in $Q^{2}$ ) and within each $Q^{2}$ bin, they were further divided into $\mathrm{W}$ bins of size $20 \mathrm{MeV}$ each. The results on $g_{1}$ and $A_{1} F_{1}$ that came from two beam energy data sets were further combined into a single set of energy independent data points. Finally, within each $Q^{2}$ bin, the newly extracted $g_{1}$ and $A_{1} F_{1}$ values were used to evaluate three integrals. All of these results are shown and described below.

\subsection{EXTRACTED $g_{1}$ AND $A_{1} F_{1}$}

Figures 90 and 91 show the extracted values of $g_{1}$ and their errors from two different beam energies $(1.337 \mathrm{GeV}$ and $1.989 \mathrm{GeV})$.It can be seen that the two energies give results that are in good agreement (in the overlapping kinematic regions).

These results from low $Q^{2}$ measurements clearly show the resonant structure in the region $W \leq 2.0$. Especially, the $\Delta$-resonance stands out through its strongly negative signal. In addition, in the second resonance region around $\mathrm{W}=1.5 \mathrm{GeV}$ where $N^{*}(1520)$ and $N^{*}(1535)$ (also denoted by $\mathrm{D}_{11}$ and $\mathrm{S}_{13}$ respectively) overlap, we see a drastic transition of $g_{1}$ (or cross section) from strongly negative values (not well described by the model because it is unconstrained there due to the lack of experimental data) at low $Q^{2}$ to clearly positive values at high $Q^{2}$ indicating that the dominance of the spin-flip helicity amplitude $A_{\frac{3}{2}}^{T}$ on cross section drastically diminishes with $Q^{2}$ and the non-flip amplitude $A_{\frac{1}{2}}^{T}$ becomes stronger (see Eq. 35). We have pushed the lower limit on $Q^{2}$ in the resonance region with reduced systematic and statistical errors that will contribute greatly to the world data set. Our data will help MAID and other phenomenological models to better constrain their parameters enabling them to make better predictions in the future. 
Likewise, Figs. 92 and 93 shows the extracted values of $A_{1} F_{1}$ and their errors from two different beam energies (1.337 GeV and $1.989 \mathrm{GeV})$. These values also show similar behavior as $g_{1}$.

Figs. 94, 95, 96 and 97 show the values of $g_{1}$ and $A_{1} F_{1}$ and their errors after combining the corresponding results from the two different beam energies as described in the previous chapter. 

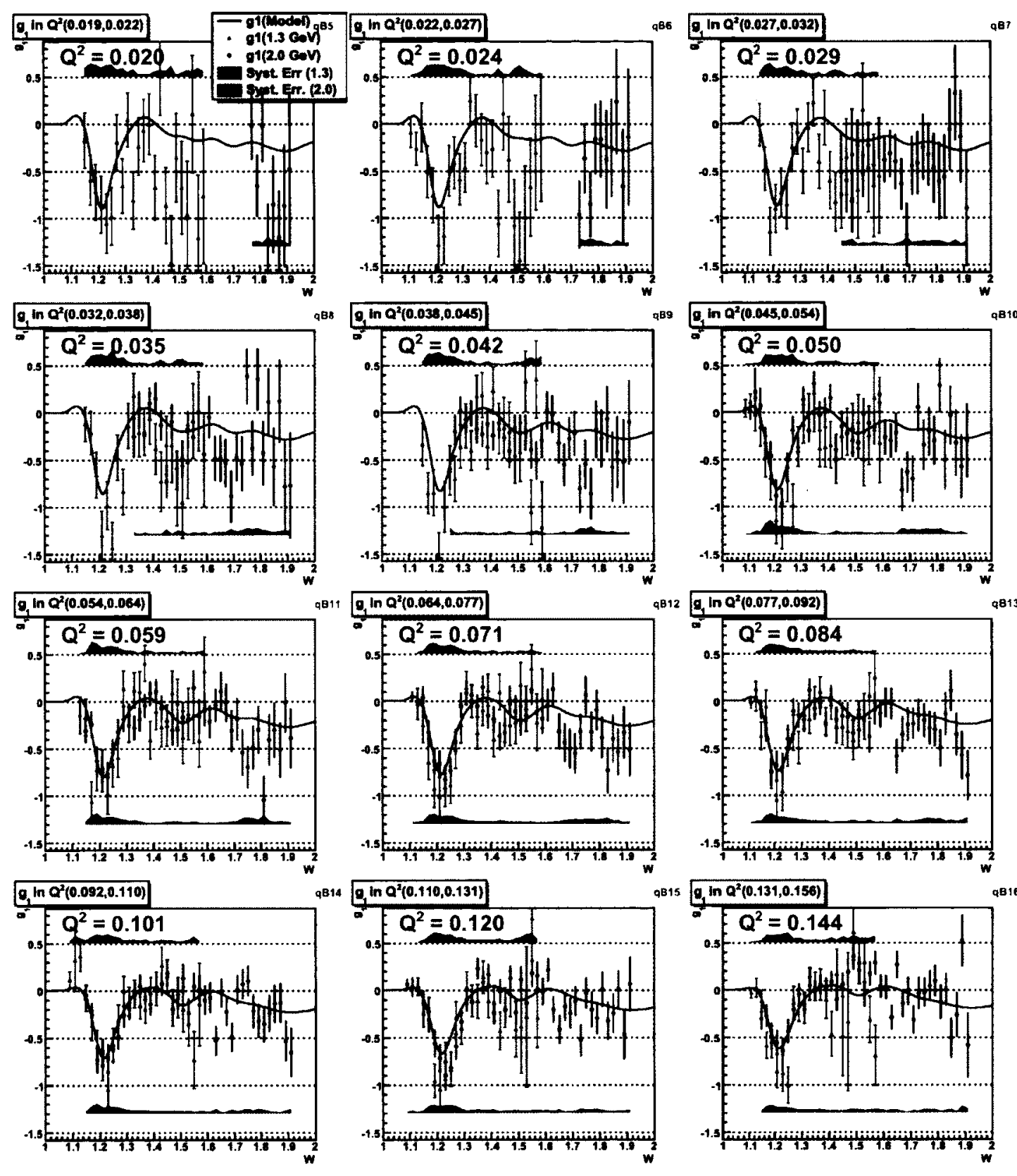

FIG. 90. Extracted $g_{1}$ for deuteron (in the first $12 Q^{2}$ bins) from the two different beam energy data sets. The statistical errors are indicated by error bars, while the systematic uncertainties are given by the bands (cyan, top: $1.3 \mathrm{GeV}$ and green, bottom: $2 \mathrm{GeV}$ ). 


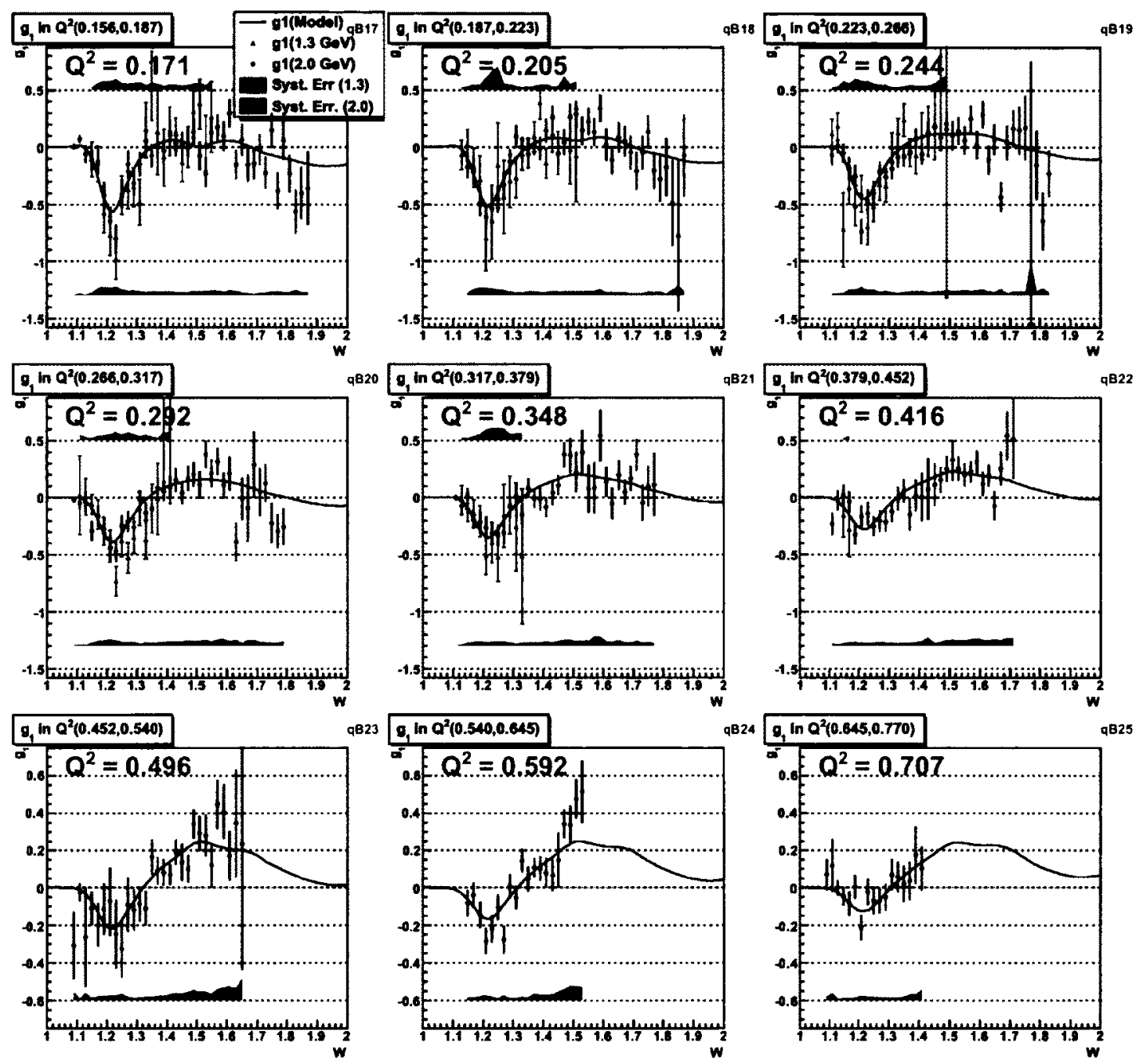

FIG. 91. Extracted $g_{1}$ for deuteron (in the last $9 Q^{2}$ bins (see Fig. 90 for the first 12 bins)) from the two different beam energy data sets. 

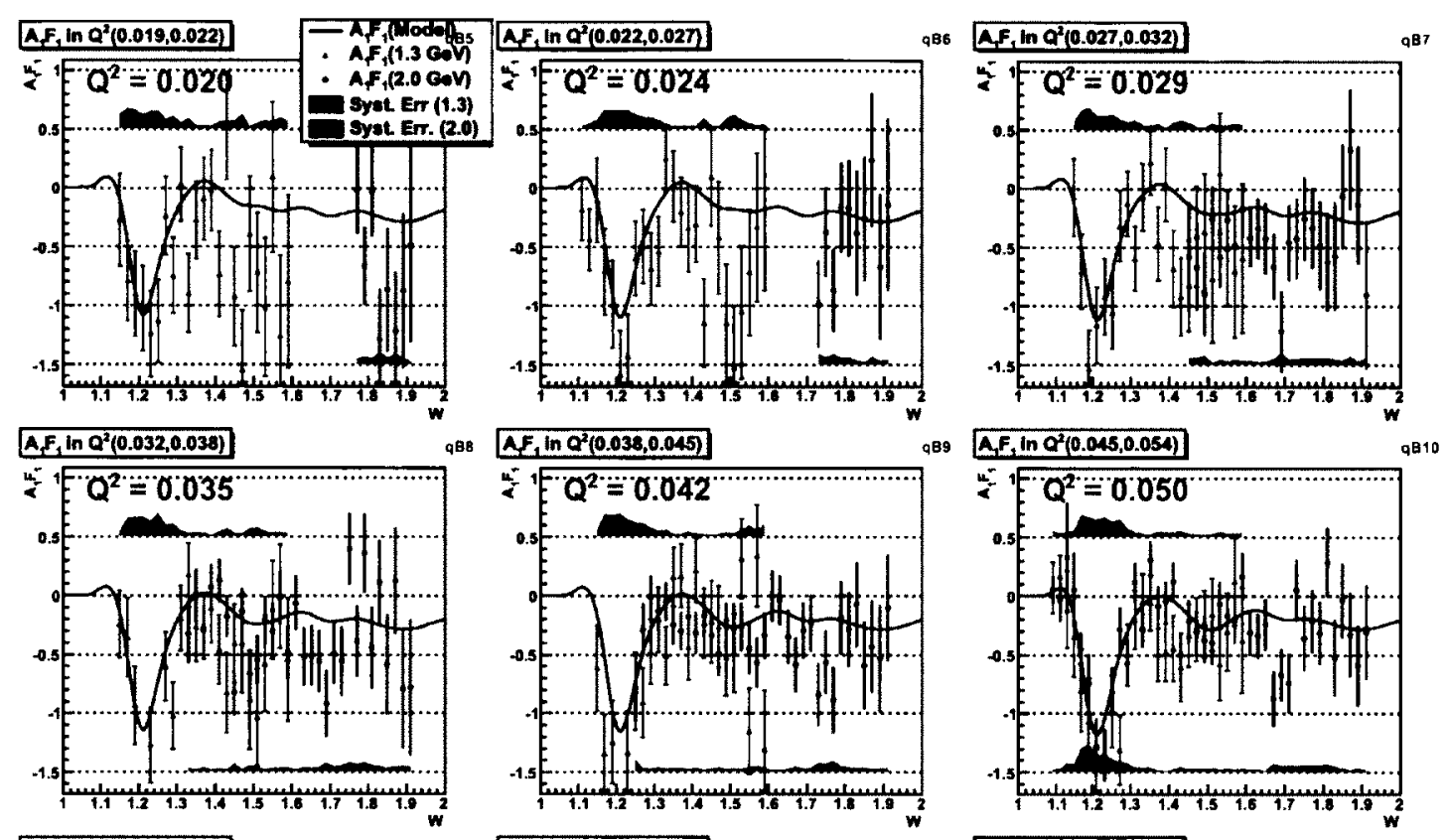

${ }_{488} A, \ln \alpha^{2}(0.038,0.045$

${ }_{989}$

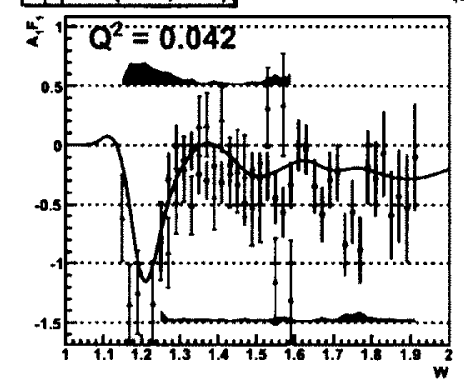

$A, E, \ln Q^{2}(0.056,0.064)$

${ }_{9811} A_{1}, \ln \alpha^{2}(0.064,0.07 \pi$
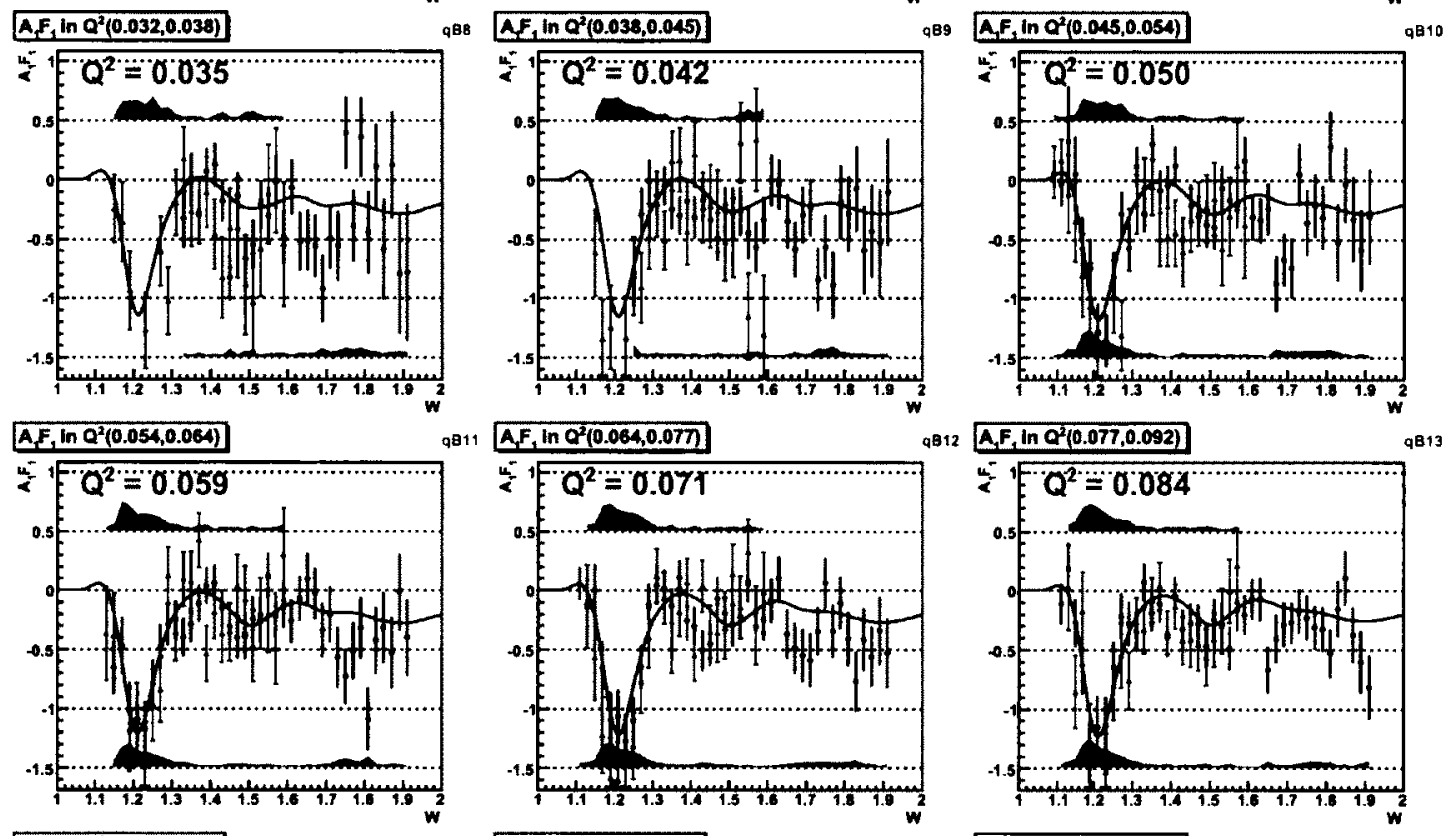

$A F_{1} \ln a^{2}(0.092,0.110)$
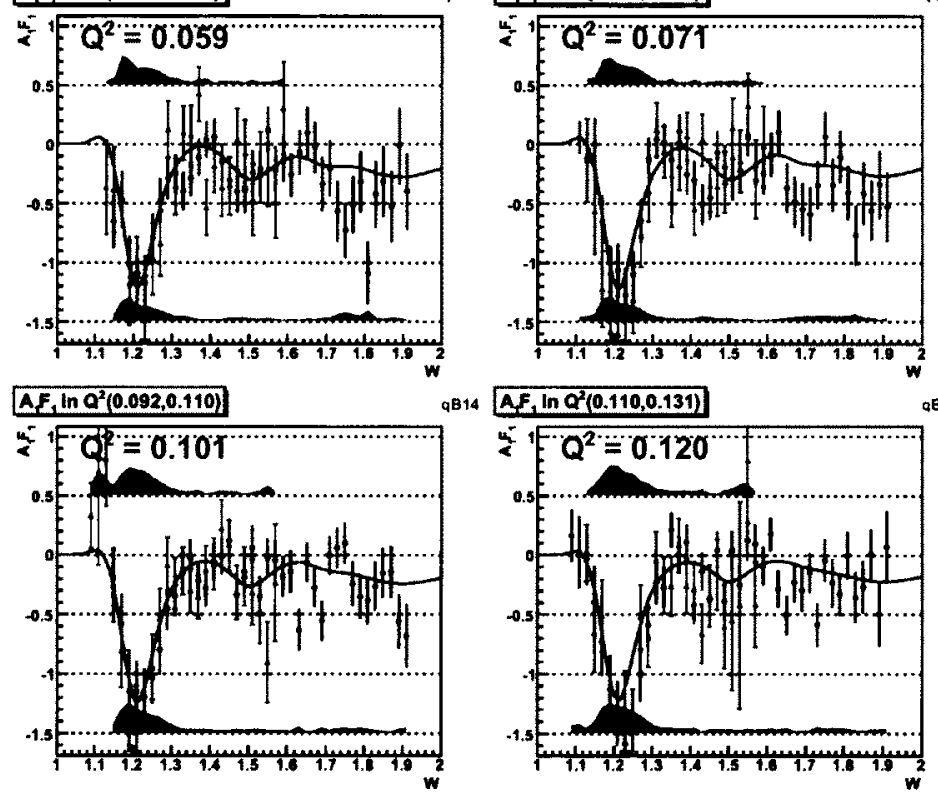

${ }_{9 B 14} A F_{1} \ln \alpha^{2}(0.110,0.131]$

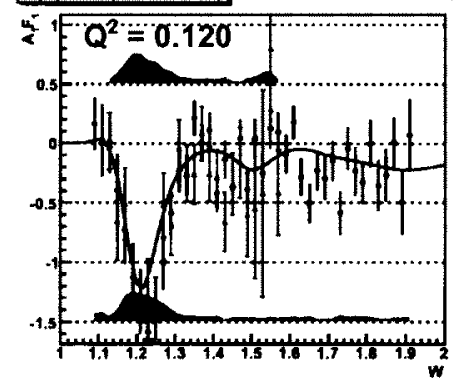

${ }_{9812}{ }_{A, F, \ln \alpha^{2}[0.077,0.092]}$

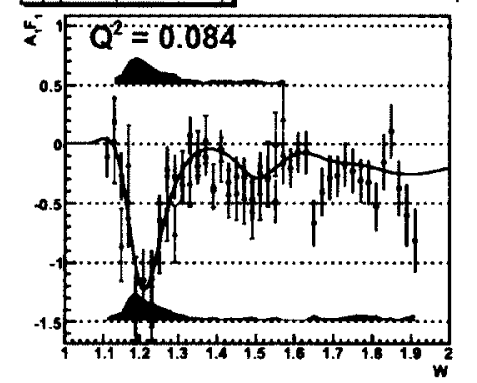

Q815 $A, F, \ln \alpha^{2}(0.131,0.156]$

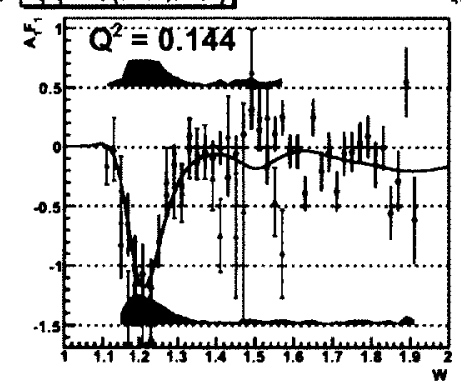

FIG. 92. Extracted $A_{1} F_{1}$ for deuteron (in the first $12 Q^{2}$ bins) from the two different beam energy data sets. The statistical errors are indicated by error bars, while the systematic uncertainties are given by the bands (cyan, top: $1.3 \mathrm{GeV}$ and green, bottom: $2 \mathrm{GeV}$ ). 

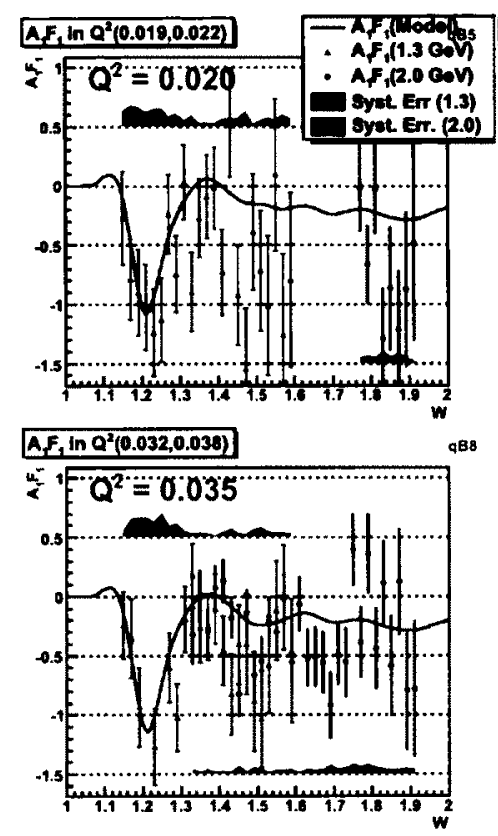

$A, F, \ln Q^{2}(0.054,0.004)$

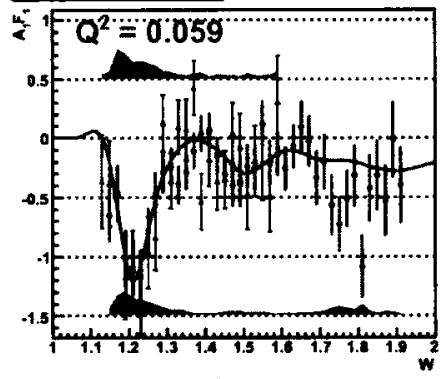

$A E_{1} \ln Q^{2} 20.092,0.110$

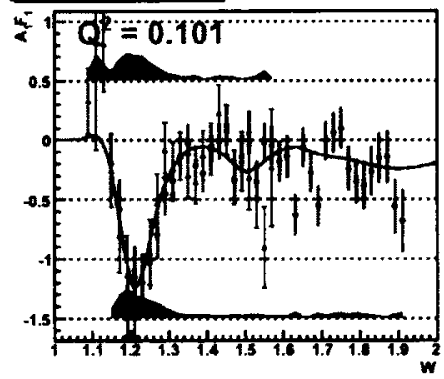

$A E_{1} \ln a^{2}(0.022,0.027$

$Q^{2}=0.024$

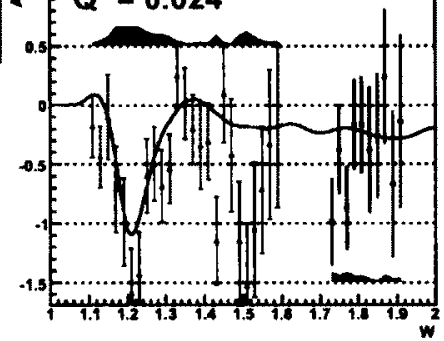

488 $A_{1} \ln \alpha^{2}(0.038,0.045$

$Q^{2}=0.042$

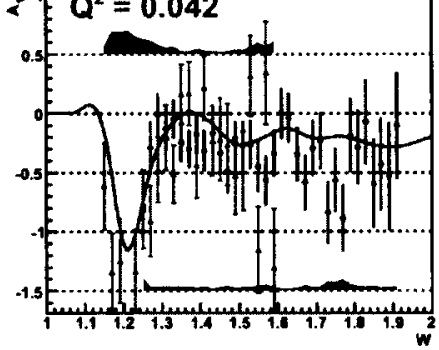

Q811 AF, in $\alpha^{2}(0.064,0.077$

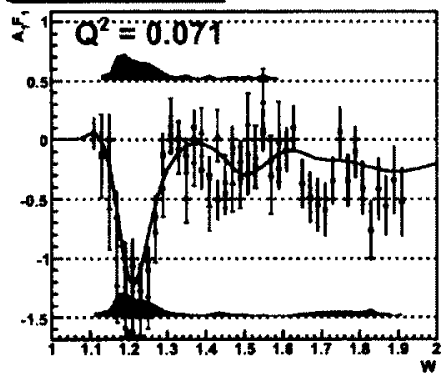

QB14 $A=\ln \alpha^{2}[0.110,0.131]$

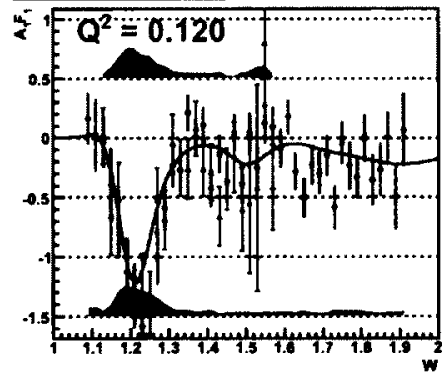

${ }_{986}{ }_{0,5, \ln \alpha^{x}(0.027,0.032]}$

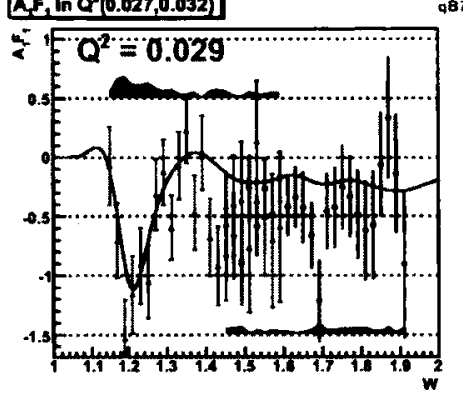

${ }_{989} A_{1,1} \ln \alpha^{2}(0.045,0.054]$

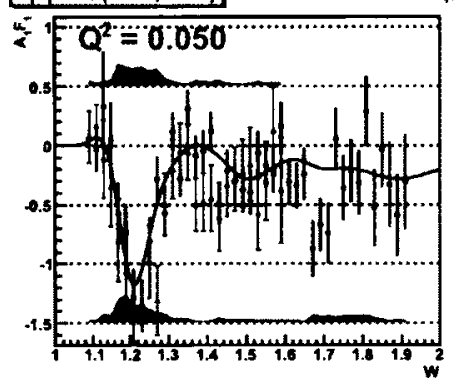

${ }_{Q B 12} A_{1, F} \ln \alpha^{2}(0.077,0.092)$

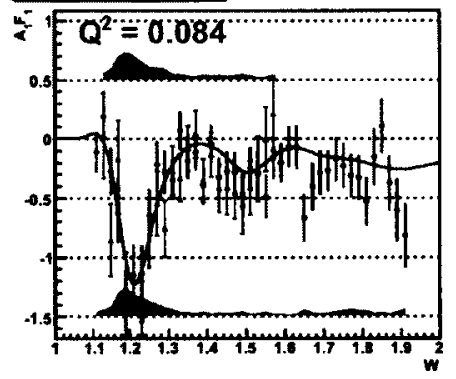

Q815 $\Delta, F_{1} \ln \alpha^{2}(0.131,0.150)$

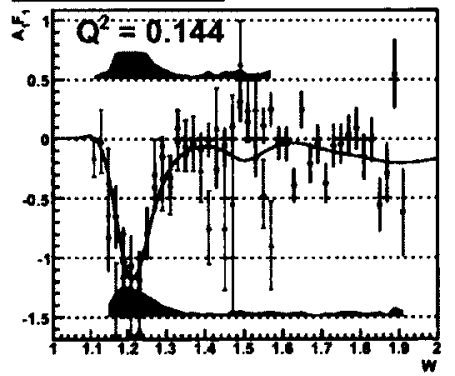

FIG. 93. Extracted $A_{1} F_{1}$ for for deuteron (in the last $9 Q^{2}$ bins (see Fig. 92 for the first 12 bins)) from the two different beam energy data sets.. 

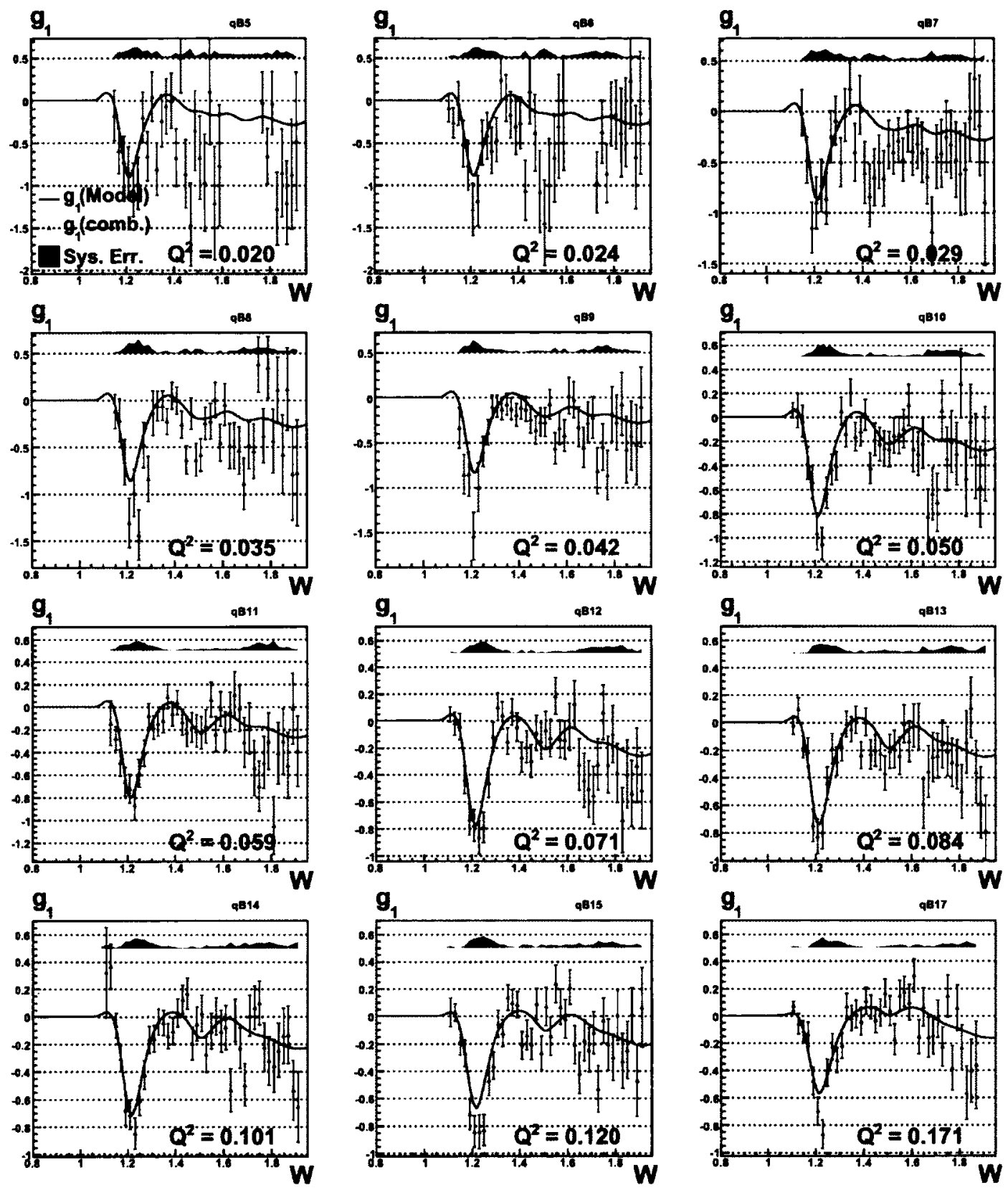

FIG. 94. Extracted $g_{1}$ for deuteron after combining the results from the two beam energies (in the first $12 Q^{2}$ bins). The red data points with error bars in each of the panels are the combined extracted results, the blue continuous line is the used model of $g_{1}$ and the green band represents the corresponding total systematic errors. 

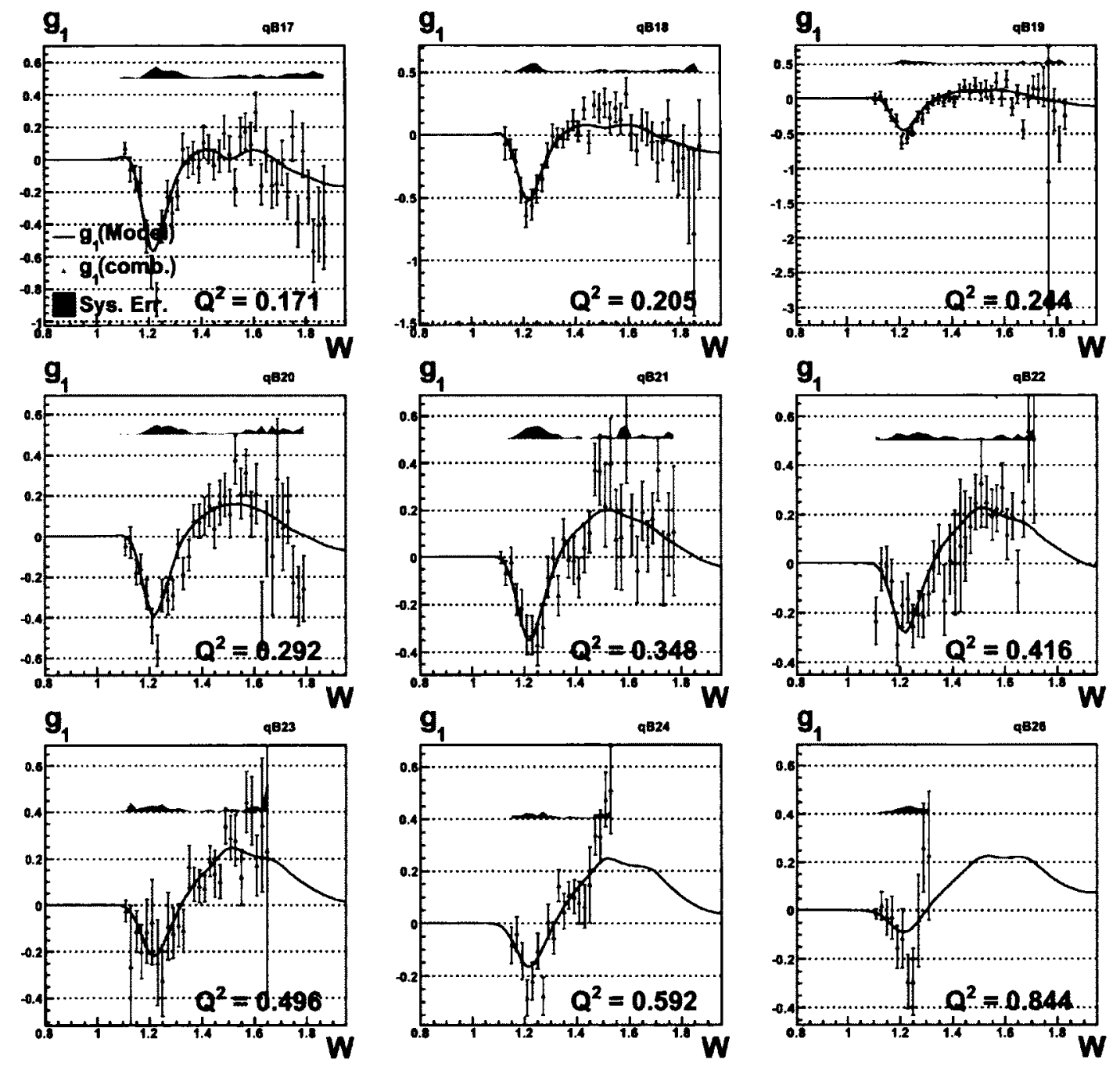

FIG. 95. Similar plots as in Fig. 94 showing the combined results on $g_{1}$ in the next $9 Q^{2}$ bins. 

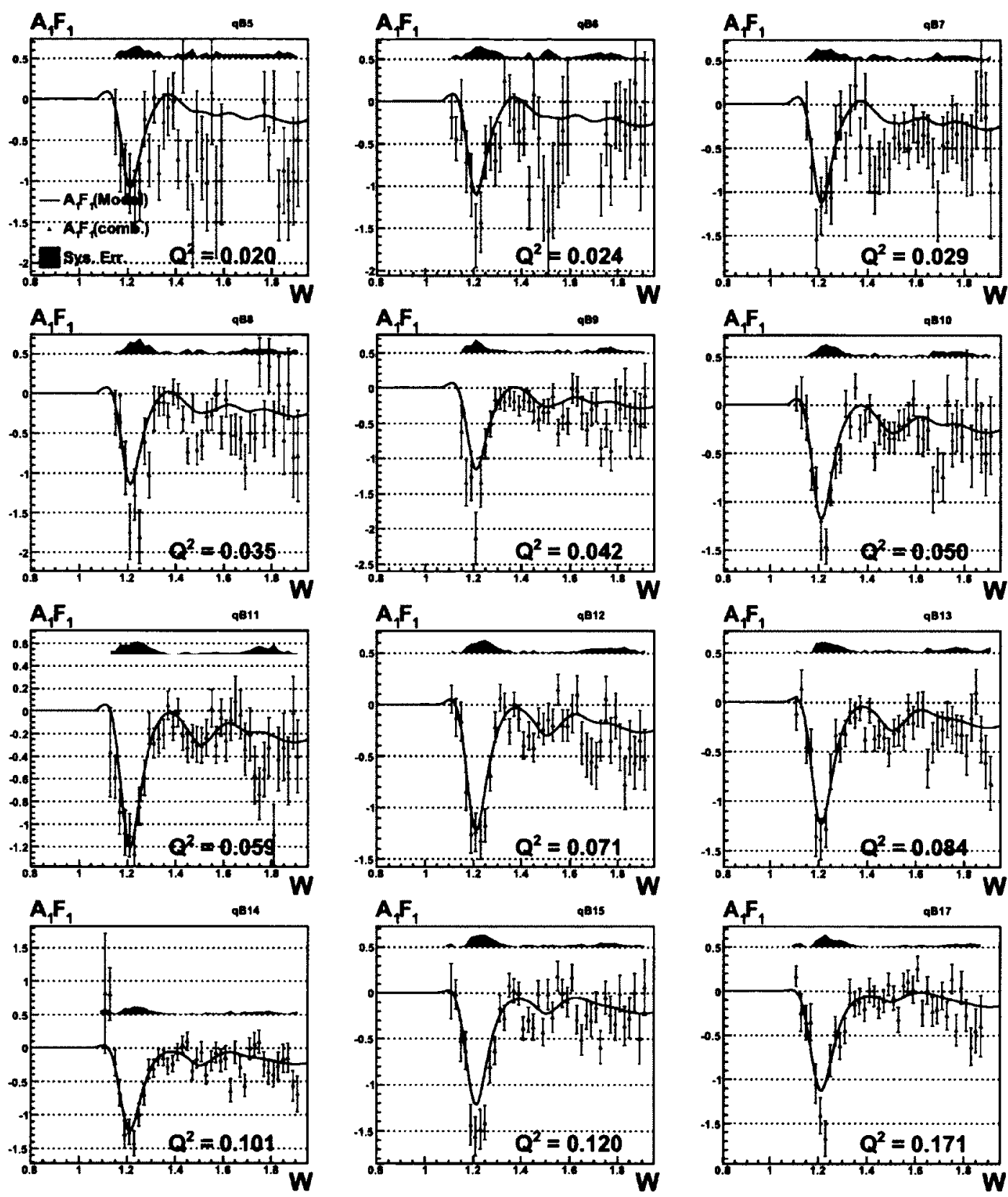

FIG. 96. $A_{1} F_{1}$ after combining the results from the two beam energies (in the first $12 Q^{2}$ bins). The red data points with error bars in each of the panels are the combined extracted results, the blue continuous line is the used model of $g_{1}$ and the green band represents the corresponding total systematic errors. 

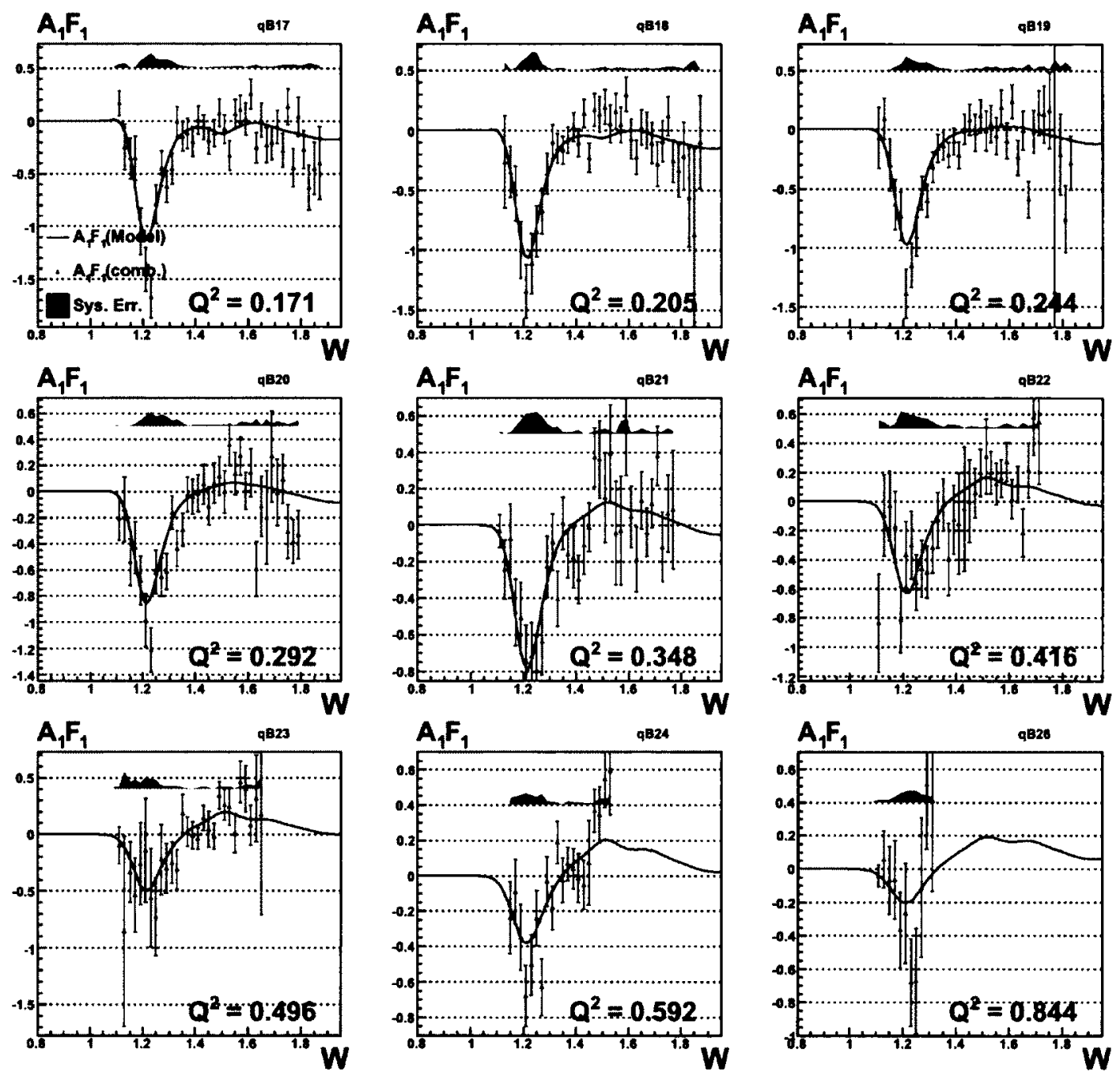

FIG. 97. Similar plots as in Fig. 96 showing the combined results on $g_{1}$ in the next $9 Q^{2}$ bins. 


\subsection{MOMENTS OF DEUTERON SPIN STRUCTURE FUNCTIONS}

Using the measured values of $g_{1}$ and $A_{1} F_{1}$, three integrals were evaluated for each of the $Q^{2}$ bins in which these data were measured. These integrals have been calculated in two ways - using only the new EG4 measurements, and adding model contributions to the data for regions not covered by our measurements. The integrals with the model contributions were calculated from $x=0.001$ to the onset of the resonance region (i.e. to the pion production threshold of $W \approx 1.08 \mathrm{GeV}$ ), dividing the sum into three parts for each $Q^{2}$ bin. For example, $\Gamma_{1}$ was evaluated by adding up the product $g_{1} \Delta x$ over the following three kinematic regions:

$$
\begin{array}{rlrl}
\Gamma_{1}\left(Q^{2}\right) & =\int_{x=0.001}^{x\left(W_{\text {data }}\right)} g_{1}\left(x, Q^{2}\right) d x & \text { model } \\
& +\int_{x=1.15}^{W\left(W_{\text {data }}\right)} g_{1}\left(x, Q^{2}\right) d x & & \text { data (or model for gaps) } \\
& +\int_{W=1.15}^{W=1.08} g_{1}\left(x, Q^{2}\right) d x & & \text { model }
\end{array}
$$

where $W_{\text {data }}$ indicates the upper edge of the last $W$ bin in which the EG4 data is available in a given $Q^{2}$ bin (the $W$ variable was divided into 70 bins of size $20 \mathrm{MeV}$ in the range $\mathrm{W}=(0.7,2.1) \mathrm{GeV})$. The first part of the integral as given by Eq. 152 is evaluated by using the model values of $g_{1}$ and using $\Delta x$ corresponding to a W bin of size $10.0 \mathrm{MeV}$ (The $\Delta W$ is converted to $\Delta x$ by using $x=Q^{2} /\left(Q^{2}+W^{2}-M^{2}\right)$ to evaluate $x$ at the two edges of each $W$ bin and taking the difference.). The second part given by Eq. 153 is evaluated similarly but using the EG4 results for $g_{1}$ if there is no measurement gap in between. If there is any gap, the same method as in the first part is used to get a model contribution for the gap and added to the data contribution. Lastly, the the third contribution given by Eq. 153 again were evaluated from from model values (quasi-elastic part turned off from the model in all of these cases) but with finer $\mathrm{W}$ bins $(1 \mathrm{MeV})$ because the integrals are very sensitive to the region near the $\Delta$ resonance due to the fact that the structure functions show rapid changes in this region. The reason to calculate the third integral using model values rather than data values is to avoid having contributions in the integrals from the quasi-elastic contamination.

The statistical errors are evaluated by adding the statistical error contribution in each $W$ or $x$ bin in quadrature. For example, if the integral is evaluated in a $Q^{2}$ bin by calculating the sum $\left(\sum_{W \text { bins }} g_{1} \cdot \Delta x\right)$, then the corresponding statistical error is evaluated by 
calculating $\sqrt{\sum_{W} g_{1} \cdot \Delta x}$. Because, the model contribution is assumed to have no statistical uncertainties, the statistical errors in the integrals come solely from the propagation of the statistical error of the measured $g_{1}$ or $A_{1} F_{1}$.

The other two integrals and their errors are evaluated in the same manner, with $g_{1}$ replaced by their corresponding integrands and again calculating the three parts of the integrals separately.

These integrals are then compared with the latest available predictions from different theories (mainly $\chi \mathrm{PT}$ ) and phenomenological calculations along with EG1b or DIS data whenever applicable.

\subsubsection{FIRST MOMENT OF $g_{1}\left(\Gamma_{1}\right)$}

The first integral of interest is the first moment of $g_{1}$ i.e., $\Gamma_{1}$ (see Eq. 61), which was calculated for all $Q^{2}$ bins for which the new data are available. Figs. 98 and 99 show the two calculations (with and without model input) along with EG1b data and several $\chi$ PT and model predictions. One important observation here is that our measurements provide the only data points in the very low $Q^{2}$ region (i.e for $Q^{2}<0.05 \mathrm{GeV}^{2}$ ) where $\chi \mathrm{PT}$ is thought to be able to make rigorous calculations. Therefore, our data will provide important benchmarks for the future calculations in this kinematics. Particularly, the latest $\chi$ PT prediction by Bernard et al. [37] seems to agree remarkably well in the very low $Q^{2}$ region.

While all other higher $Q^{2}$ predictions, except that of $\mathrm{Ji}$ et al., seem to be within the uncertainties of our measurements, it can be seen that the phenomenological predictions of Soffer et al. compare slightly better with data than others (excluding, of course, the Bernard et al. prediction).

\subsubsection{THE EXTENDED GDH INTEGRAL $\bar{I}_{T T}$}

Using the measured values of $A_{1} F_{1}$, the generalized GDH integral $\bar{I}_{T T}=2 M^{2} / Q^{2} \int A_{1} F_{1}\left(x, Q^{2}\right) d x$ was also calculated and compared (see Figs. 100 and 101) with the latest $\chi$ PT calculation from Bernard et al. [37]. We can see that at the very low $Q^{2}$, the $\chi$ PT prediction and the measurement get very close. The $\chi \mathrm{PT}$ methods determine the higher powers of $Q^{2}$ in the Taylor expansion of the integral around the photon point $Q^{2}=0$, beyond the prediction of the GDH sum rule which determines the lowest order term. Our data seem indeed to converge towards the GDH sum rule at our lowest $Q^{2}$. However, only one or two higher order terms can be calculated confidently, since higher orders require additional (unknown) 


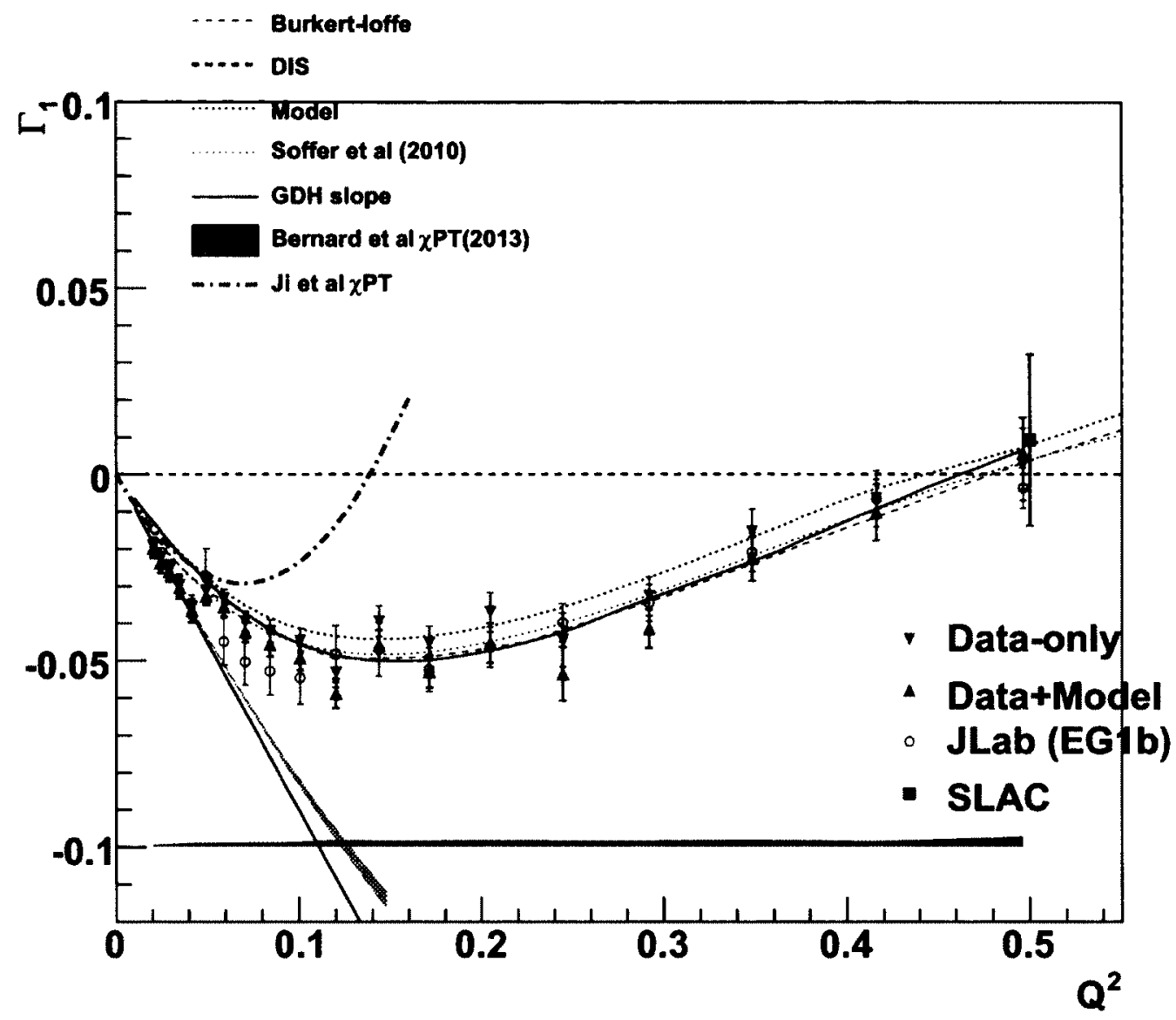

FIG. 98. Extracted $\Gamma_{1}$ for deuteron compared with some of the past measurements and various theoretical predictions with a linear scale used for $Q^{2}$.

constants. Therefore, $\chi$ PT predictions do reasonably well at ultra-low $Q^{2}$ but cannot be expected to work at the higher $Q^{2}$, where the data show a turn-around and a transition towards positive values.

\subsubsection{THE GENERALIZED FORWARD SPIN POLARIZABILITY $\gamma_{0}$}

Finally, the generalized forward polarizability (as given by Eq. 65) for the deuteron was also calculated using the measured values of $A_{1} F_{1}$ and then compared with various predictions as shown in Figs. 102 and 103. The comparison shows that both $\chi$ PT calculations by Bernard et al. and Kao et al. converge with data at the lowest $Q^{2}$ bins. The MAID prediction is shown for reference but seems to be somewhat off the current results. 


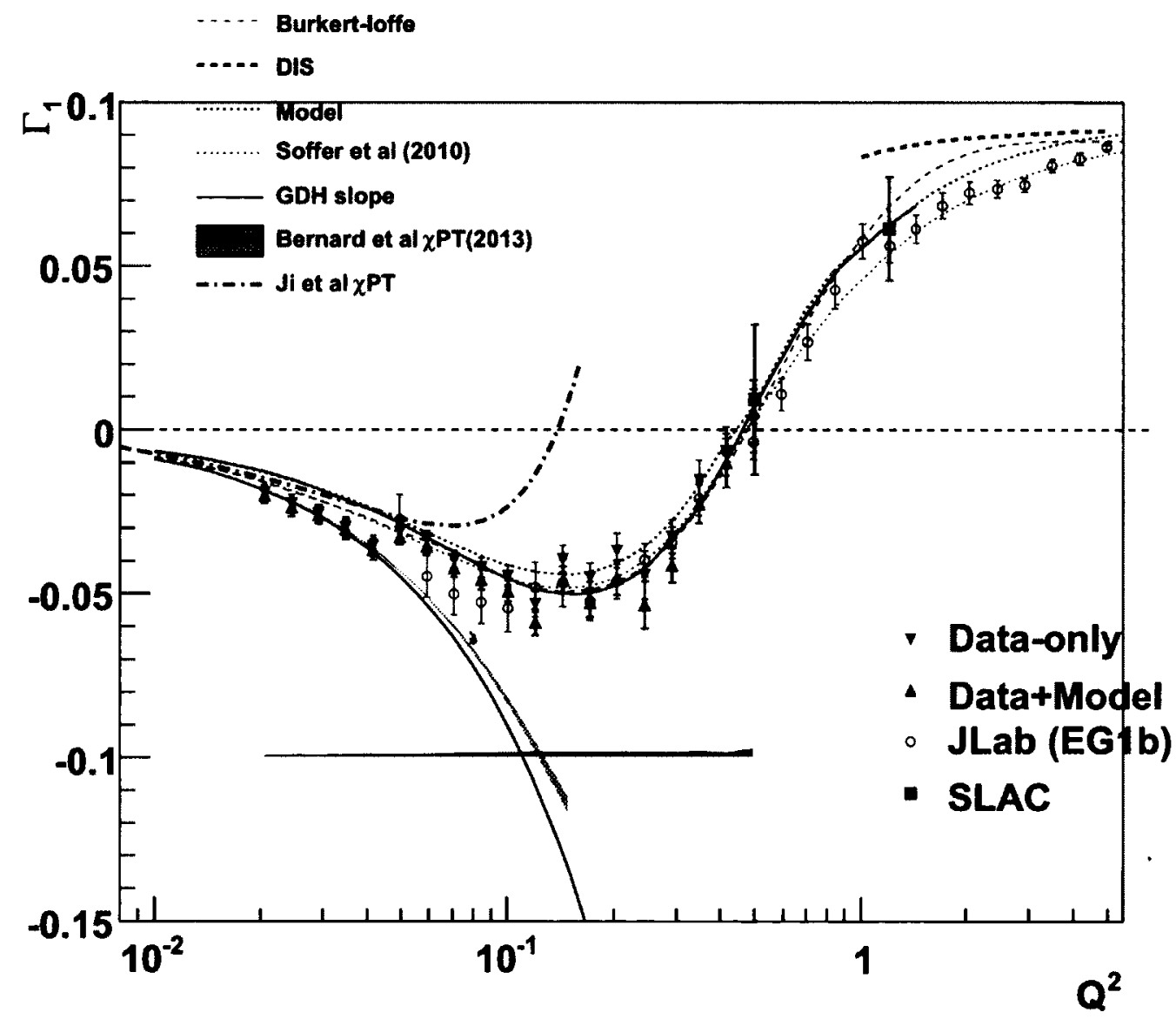

FIG. 99. Extracted $\Gamma_{1}$ for deuteron compared with some of the past measurements and various theoretical predictions with a logarithmic scale used for $Q^{2}$. 


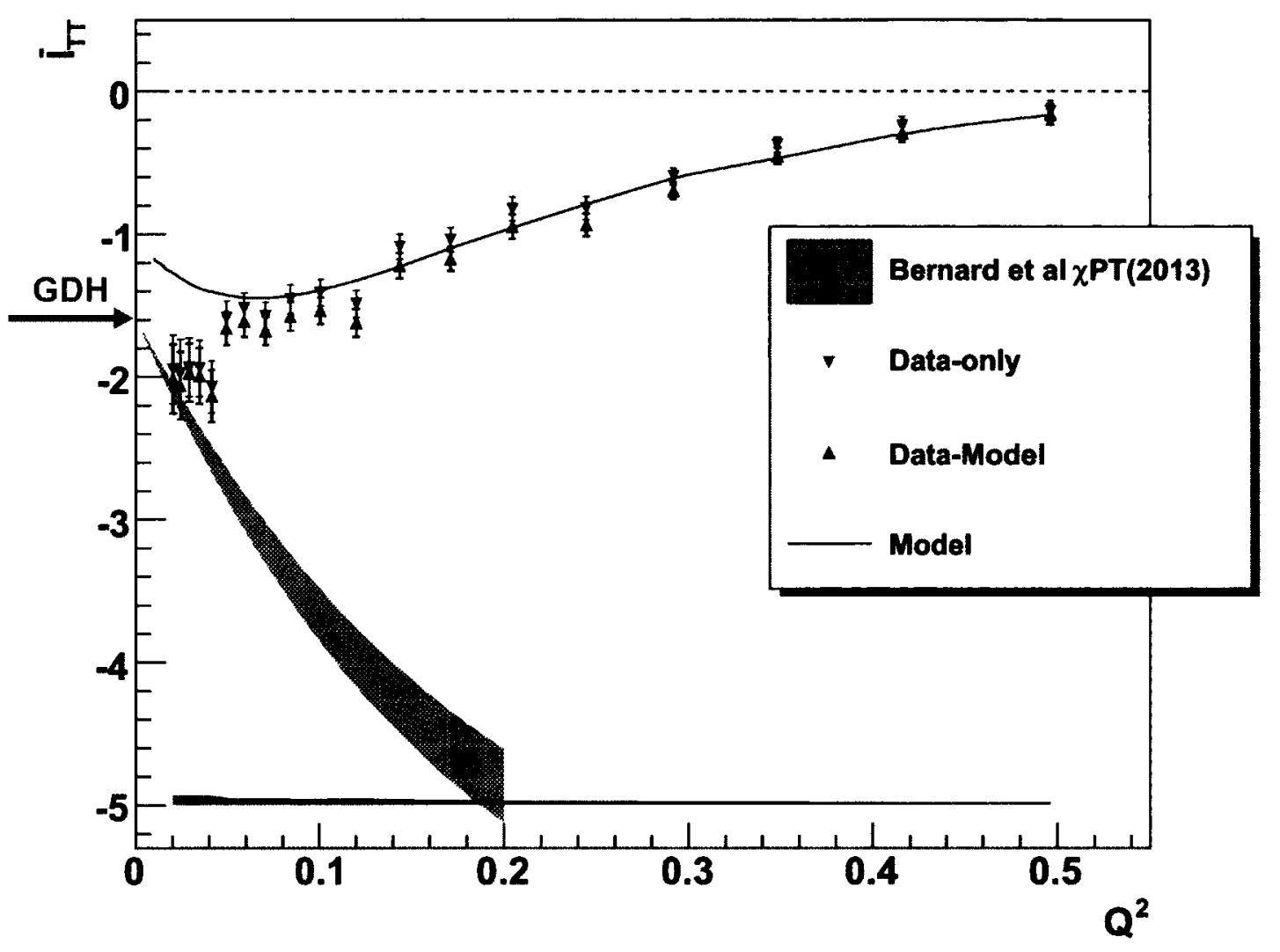

FIG. 100. Extracted $\bar{I}_{t t}$ for deuteron compared with the used model and a $\chi$ PT prediction with a linear scale used for $Q^{2}$. 


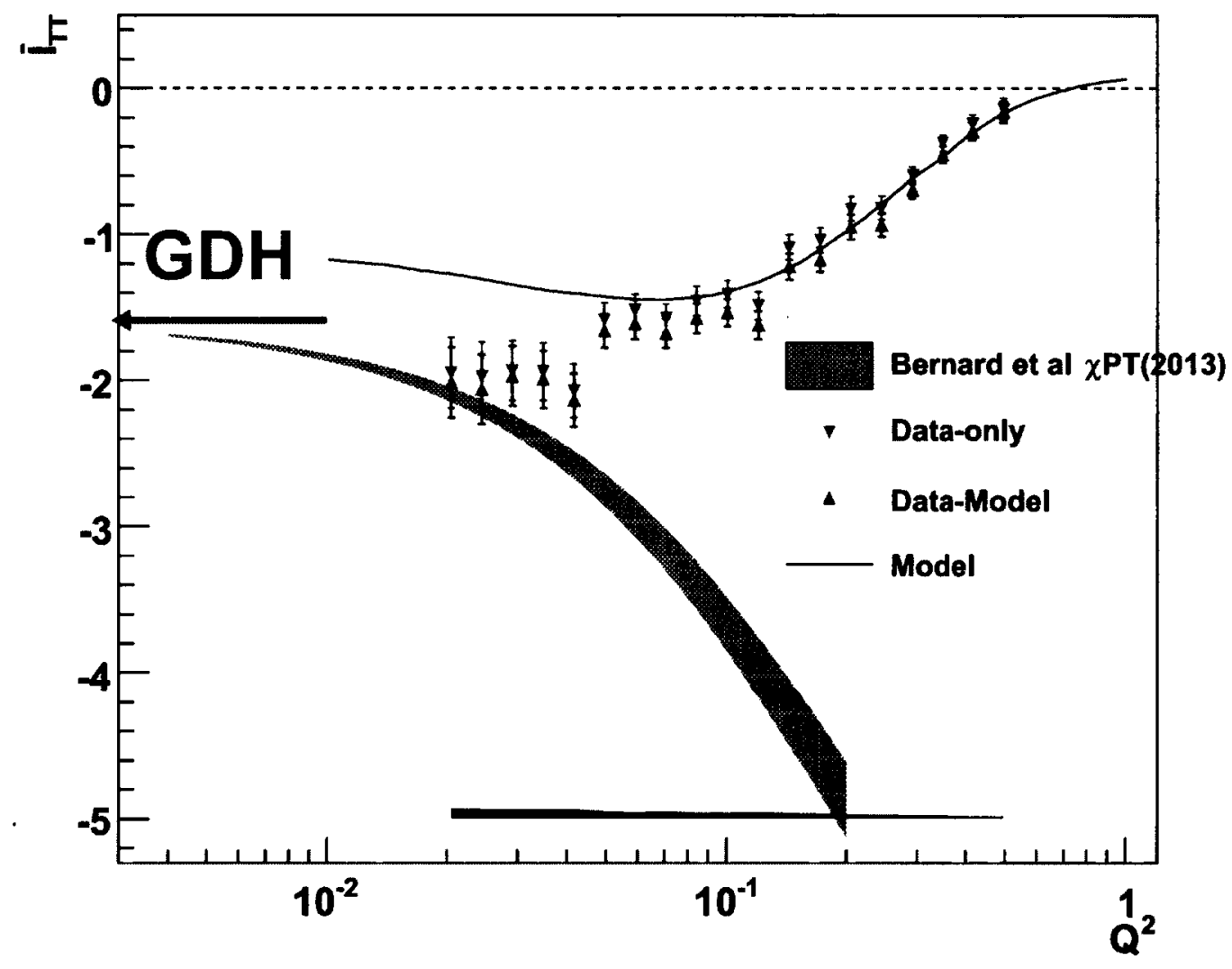

FIG. 101. Extracted $I_{t t}$ for deuteron compared with the used model and a $\chi$ PT prediction with a logarithmic scale used for $Q^{2}$. 


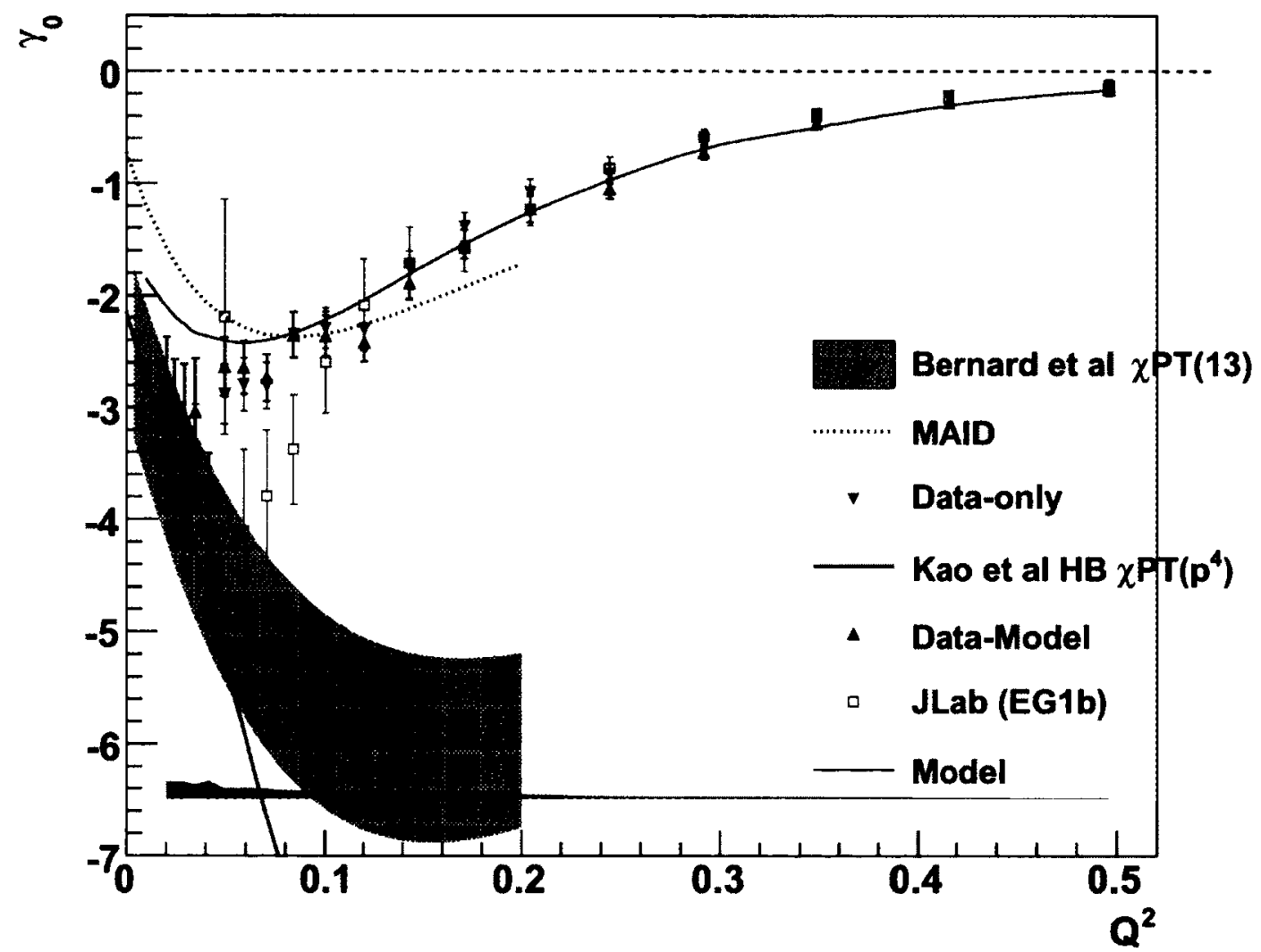

FIG. 102. Extracted $\gamma_{0}$ for deuteron compared with some of the past measurements and various theoretical predictions with a linear scale used for $Q^{2}$. 


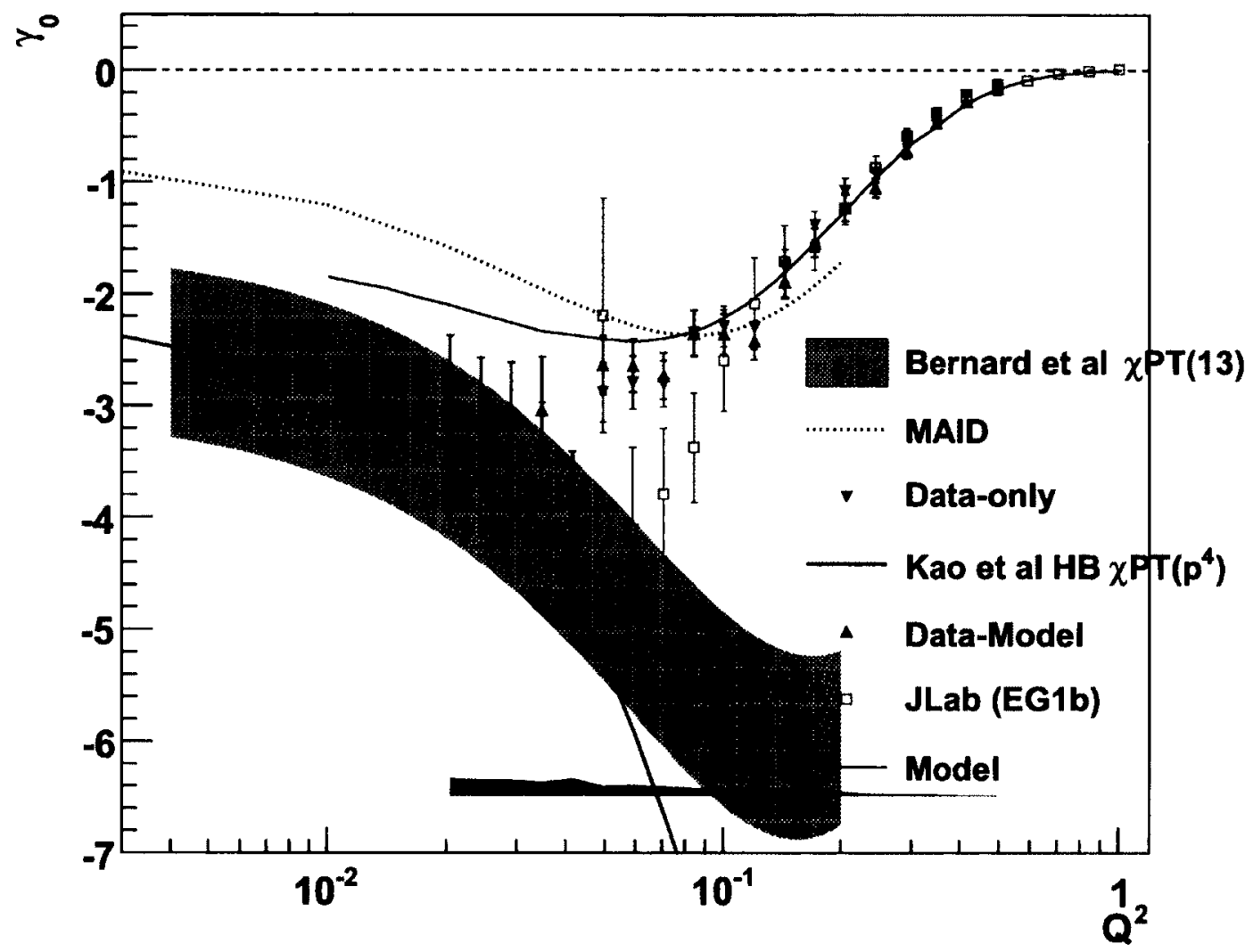

FIG. 103. Extracted $\gamma_{0}$ for deuteron compared with some of the past measurements and various theoretical predictions with a logarithmic scale used for $Q^{2}$. 


\section{CHAPTER 6}

\section{CONCLUSION}

The EG4 experiment collected a large amount of very low momentum transfer $\left(Q^{2}\right)$ data for the helicity dependent inclusive cross section (difference) for the scattering of longitudinally polarized electrons off longitudinally polarized protons and deuterons (from DNP polarized $\mathrm{NH}_{3}$ and $\mathrm{ND}_{3}$ targets respectively). The use of low beam energies (1.03.0 GeV) (from CEBAF accelerator) and the modified CLAS detector optimized for low scattering angle measurements (down to 6 degrees), allowed data collection at an unprecedented level of precision and low $Q^{2}$ coverage. The deuteron data (collected using 1.337 and $2.0 \mathrm{GeV}$ beam energies) which is the subject of this thesis has the kinematic coverage of $\left(0.02 \mathrm{GeV}^{2}<Q^{2}<0.7 \mathrm{GeV}^{2}\right)$ and $\left(1.08 \mathrm{GeV}<W<2.0 \mathrm{GeV}^{2}\right)$. Although, past measurements from EGlb go as low as $0.05 \mathrm{GeV}^{2}$ in $Q^{2}$, the new measurements have higher precision (due to higher statistics and better detection efficiency) in the overlapping region in addition to new high precision data in the previously unmeasured lower $Q^{2}$ region.

The new deuteron data were used to extract the deuteron's spin structure function $g_{1}$ by comparing the experimental data with simulated data produced by using a realistic cross section model for the deuteron under similar kinematic conditions. The newly extracted data pushes the lower limit on $Q^{2}$ in the resonance region with reduced systematic and statistical uncertainties that will contribute greatly to the world data set. It is observed that the data from two beam energies give results that are in good agreement. The low $Q^{2}$ results clearly show resonance structure in the region $W \leq 2.0$ which smooths out as $Q^{2}$ becomes larger. In particular, the $\Delta$-resonance shows a strongly and consistently negative signal at all $Q^{2}$, but the second resonance region (around $\mathrm{W}=1.5 \mathrm{GeV}$ ) shows a rather unexpected rapid transition of $g_{1}$ (or cross section) from strongly negative values at low $Q^{2}$ to clearly positive values at high $Q^{2}$. is not well described by the model because it is not constrained in the region due to the lack of experimental data and indicates that the spin-flip helicity amplitude $A_{\frac{3}{2}}^{T}$ dominates the cross section at low $Q^{2}$ while the non-flip amplitude $A_{\frac{1}{2}}^{T}$ becomes stronger at higher $Q^{2}$. 
The product $A_{1} F_{1}$ of the virtual photon asymmetry $A_{1}$ and the unpolarized structure function $F_{1}$ was also extracted from the same data and method. The extracted results on $g_{1}$ and $A_{1} F_{1}$ were then used to evaluate the following three important moments - the first moment $\Gamma_{1}^{d}$ of $g_{1}$, the generalized GDH integral $\bar{I}_{T T}^{d}$ and the generalized forward spin polarizability $\gamma_{0}^{d}$ - in each of the $Q^{2}$ bins in which the new $g_{1}$ and $A_{1} F_{1}$ have been extracted. The new low $Q^{2}$ measurements of the moments evaluated both with and without model inputs for the unmeasured kinematic regions were then compared with various $\chi \mathrm{PT}$ calculations, phenomenological predictions and past measurements, particularly the EGlb or DIS data whenever applicable.

The EG4 results provide the only data points in the very low $Q^{2}$ region (i.e for $Q^{2}<$ $0.05 \mathrm{GeV}^{2}$ ) where $\chi \mathrm{PT}$ is thought to be able to make rigorous calculations. The high precision data will provide important benchmarks for the future calculations in this kinematics. In the case of the first moment $\Gamma_{1}^{d}$, the EG4 results show remarkable agreement with the latest $\chi$ PT prediction by Bernard et al. [37] in the very low $Q^{2}$ region. The phenomenological predictions which have much larger $Q^{2}$ coverage also seem to agree within the uncertainties of our measurements, with the predictions of Soffer et al. showing slightly better comparison than others. Likewise, the very low $Q^{2}$ results of the generalized GDH integral $\bar{I}_{T T}$ are indeed observed to converge towards the GDH sum rule and thus getting very close to the $\chi \mathrm{PT}$ predictions by Bernard et al. [37]. Finally, the generalized forward polarizability $\left(\gamma_{0}^{d}\right)$ for the deuteron calculated from the EG4 data and the $\chi$ PT calculations by Bernard et al. and Kao et al. seem to converge at the lowest $Q^{2}$ bins. The MAID prediction, however, seems to be somewhat off the current results.

The deuteron data in combination with the EG4 proton data taken under similar conditions (currently being analyzed by another collaborator and results expected to come very soon) will be useful in extracting neutron quantities in near future, which is valuable because of the unavailability of the free neutron targets. Moreover, due to the complexities of the nuclear medium effects, neutron data from deuteron will be very important to enhance confidence in similar neutron results extracted from other nuclear targets particularly ${ }^{3} \mathrm{He}$.

The new data on spin structure functions will help MAID and other phenomenological models to better constrain their parameters enabling them to make better predictions in the future. With the availability of the high precision data in the previously (largely) unmeasured region that has the potential to help constrain the theories and models, it is hoped that a unified description of spin structure functions over all kinematic regions will be possible in future. 


\section{BIBLIOGRAPHY}

[1] D. R. Smith, C. Truesdell, An introduction to continuum mechanics - after Truesdell and Noll (Kluwer Academic Publishers, P.O. Box 17,3300 AA Dordrecht, The Netherlands, 1993) ISBN 0-7923-2454-4

[2] D. J. Griffiths, Introduction to Quantum Mechanics, 2nd ed. (Pearson Prentice Hall, Upper Saddle River, NJ 07458, 2005) pp. 183-4

[3] W. Gerlach and O. Stern, Zeitschrift fr Physik 9, 353 (1922), ISSN 0044-3328

[4] D. Drechsel and L. Tiator, GDH 2000: Proceedings of the Symposium on the Gerasimov-Drell-Hearn Sum Rule and the Nucleon Spin Structure in the Resonance Region : Mainz, Germany, 14-17 June 2000 (World Scientific, 2001) ISBN 9789810245740

[5] S. Kuhn, in Proceedings of the 12th Annual HUGS at CEBAF (1997) article based on six lectures on the subject during the 1997 Hampton University Graduate Summer School.

[6] J. Aubert et al., Physics Letters B 123, 275 (1983), ISSN 0370-2693

[7] K. Gottfried, Phys. Rev. Lett. 18, 1174 (Jun 1967)

[8] M. Arneodo et al., Phys. Rev. D 50, R1 (Jul 1994)

[9] J. Ashman and other, Physics Letters B 206, 364 (1988), ISSN 0370-2693

[10] O. Piguet, G. Pollak, and M. Schweda, Nucl.Phys. B328, 527 (1989)

[11] K. G. V. G. Dharmawardane, Spin Structure Functions of the Deuteron Measured with CLAS in and above the Resonance region, Ph.D. thesis, Old Dominion University (May 2004)

[12] D. Gross and F. Wilczek, Phys. Rev. Lett. 30, 1343 (1973)

[13] J. P. Chen and others, "The GDH Sum Rule and the Spin Structure of ${ }^{3} \mathrm{He}$ and the Neutron using Nearly-Real Photons,' JLab Hall A Proposal (1997), PR97-110

[14] M. Hosoda and K. Yamamoto, Prog. Theor. Phys. 30, 425 (1966) 
[15] K. Slifer, A Measurement of the Extended GDH Integral and the BurkhardtCottingham Sum Rule, Ph.D. thesis, Temple University (August 2004)

[16] M. Anselmino et al., in Proceedings of the International School of Physics "Enrico Fermi" (Varenna On Lake Como Italy, 2011)

[17] D. Drechsel, B. Pasquini, and M. Vanderhaeghen, Phys. Rept. 378, 99 (2003), arXiv:hep-ph/0212124 [hep-ph]

[18] S. Kuhn, J.-P. Chen, and E. Leader, Prog.Part.Nucl.Phys. 63, 1 (2009), arXiv:0812.3535 [hep-ph]

[19] V. A. Sulkosky, The Spin Structure of ${ }^{3} \mathrm{He}$ and the neutron at low $Q^{2}:$ A measurment of the generalized GDH integrand, Ph.D. thesis, The College of William and Mary (August 2007)

[20] C. S. B. Povh, K. Rith and F. Zetsche, Particles and Nuclei, 3rd ed. (Springer, Berlin, 2002)

[21] J. Beringer et al. (Particle Data Group), Phys. Rev. D 86, 010001 (Jul 2012)

[22] Y. L. Dokshitzer, Sov.Phys.JETP 46, 641 (1977)

[23] V. Gribov and L. Lipatov, Sov.J.Nucl.Phys. 15, 438 (1972)

[24] G. Altarelli and G. Parisi, Nucl.Phys. B126, 298 (1977)

[25] S. Gerasimov, Yad. Fiz. 2, 598 (1965)

[26] S. D. Drell and A. C. Hearn, Phys. Rev. Lett. 16, 908 (May 1966)

[27] H. Dutz et al. (GDH Collaboration), Phys. Rev. Lett. 91, 192001 (Nov 2003), http://link. aps.org/doi/10.1103/PhysRevLett.91.192001

[28] H. Dutz et al. (GDH Collaboration), Phys. Rev. Lett. 94, 162001 (Apr 2005), http://link. aps .org/doi/10.1103/PhysRevLett.94.162001

[29] D. Drechsel and L. Tiator, Ann.Rev.Nucl.Part.Sci. 54, 69 (2004), arXiv:nucl-th/0406059 [nucl-th]

[30] S. Hoblit et al. (LEGS-Spin Collaboration), Phys. Rev. Lett. 102, 172002 (Apr 2009), http://link.aps.org/doi/10.1103/PhysRevLett.102.172002 
[31] M. Anselmino, B. Ioffe, and E. Leader, Sov.J.Nucl.Phys. 49, 136 (1989)

[32] A. Deur et al., "Measurement of the Gerasimov-Drell-Hearn Integral at low $Q^{2}$ on the Neutron and Deuteron," CLAS Proposal (Dec. 2006), pR06-017

[33] M. Burkardt, AIP Conf. Proc. 1155, 26 (2009), arXiv:0905.4079 [hep-ph]

[34] M. Anghinolfi and others, "The GDH Sum Rule with Nearly-Real Photons and the Proton $g_{1}$ Structure Function at Low Momentum Transfer," CLAS Proposal (2003), PR03-006

[35] N. Guler, Spin Structure of the Deuteron, Ph.D. thesis, Old Dominion University (December 2009)

[36] C.-W. Kao, B. Pasquini, and M. Vanderhaeghen, Phys. Rev. D70, 114004 (2004), arXiv:hep-ph/0408095 [hep-ph]

[37] V. Bernard, E. Epelbaum, H. Krebs, and U.-G. Meissner, Phys. Rev. D87, 054032 (2013), arXiv: 1209.2523 [hep-ph]

[38] D. Drechsel, S. Kamalov, and L. Tiator, Eur.Phys.J. A34, 69 (2007), arXiv:0710.0306 [nucl-th]

[39] C. W. Kao, T. Spitzenberg, and M. Vanderhaeghen, Phys. Rev. D 67, 016001 (Jan 2003)

[40] V. Bernard, T. R. Hemmert, and U.-G. Meissner, Phys. Rev. D67, 076008 (2003), arXiv:hep-ph/0212033 [hep-ph]

[41] V. D. Burkert, Phys. Rev. D63, 097904 (2001), arXiv:nucl-th/0004001 [nucl-th]

[42] K. G. Wilson, Phys. Rev. 179, 1499 (Mar 1969)

[43] L. Tiator and S. Kamalov, 16(2006), arXiv:nucl-th/0603012 [nucl-th]

[44] V. Burkert and B. Ioffe, Phys. Lett. B296, 223 (1992)

[45] V. Burkert and B. Ioffe, J.Exp.Theor.Phys. 78, 619 (1994)

[46] J. Soffer and O. V. Teryaev, Phys. Lett. B545, 323 (2002), arXiv:hep-ph/0207252 [hep-ph] 
[47] R. S. Pasechnik, J. Soffer, and O. V. Teryaev, Phys. Rev. D82, 076007 (2010), arXiv:1009.3355 [hep-ph]

[48] K. G. Wilson, Phys. Rev. D 10, 2445 (Oct 1974)

[49] A. Bazavov et al., Rev. Mod. Phys. 82, 1349 (May 2010), http://link.aps .org/ doi/10.1103/RevModPhys.82.1349

[50] S. Durr, Z. Fodor, J. Frison, C. Hoelbling, R. Hoffmann, et al., Science 322, 1224 (2008), arXiv:0906.3599 [hep-lat]

[51] U.-G. Meissner(2006), arXiv:hep-ph/0610200 [hep-ph]

[52] V. Bernard and U.-G. Meissner, Ann.Rev.Nucl.Part.Sci. 57, 33 (2007), arXiv:hep-ph/0611231 [hep-ph]

[53] M. Garcon and J. Van Orden, Adv.Nucl.Phys. 26, 293 (2001), arXiv:nucl-th/0102049 [nucl-th]

[54] Y. Kahn, W. Melnitchouk, and S. A. Kulagin, Phys. Rev. C79, 035205 (2009), arXiv:0809.4308 [nucl-th]

[55] "The CEBAF accelerator," http://www.aip.org/png/html/cebaf.htm, [Online; accessed 22-Sep-2013]

[56] C. W. Leemann, D. R. Douglas, and G. A. Krafft, Annual Review of Nuclear and Particle Science 51, 413 (2001)

[57] "Jefferson lab," http://www.jlab.org/news/facts/vocab.html, [Online; accessed 22-Sep-2013]

[58] X. Zheng, Precision Measurement of Neutron Spin Asymmetry $A_{1}^{n}$ at Large $x_{B j} U s-$ ing CEBAF at $5.7 \mathrm{GeV}$, Ph.D. thesis, Massachusetts Institute of Techology (December 2002)

[59] J. Grames, D. W. Higinbotham, and H. E. Montgomery, Scholarpedia 5, 10211 (2010)

[60] Kazimi et al., in Proceedings of EPAC 2004 (Lucerne, Switzerland, 2004) ISBN ISBN 92-9083-231-2 
[61] R. G. Fersch, Measurement of Inclusive Proton Double-Spin Asymmetries and Polarized Structure Functions, Ph.D. thesis, The College of William and Mary (August 2008)

[62] R. D. Vita, Measurement of the Double Spin Asymmetry in $\pi^{+}$electroproduction with CLAS, Ph.D. thesis, University of Genova (2000)

[63] S. E. Kuhn, Private communications (2013)

[64] C. Leemann, D. Douglas, and G. Krafft, Ann.Rev.Nucl.Part.Sci. 51, 413 (2001)

[65] M. F. Spata, CERN Document Server(2001)

[66] M. Williams, Measurement of Differential Cross Sections and Spin Density Matrix Elements along with a Partial Wave Analysis for $\gamma p \rightarrow p \omega$ using CLAS at Jefferson Lab, Ph.D. thesis, Carnegie Mellon University (November 2007)

[67] R. Ursic et al., http://cdsweb.cern.ch/record/586314/files/p101.pdf, [Online; accessed 22-Sep-2013]

[68] https://hallaweb.jlab.org/wiki/index.php/Practical_Primer_on_ the_UVa_Target, [Online; accessed 22-Sep-2013], https://hallaweb.jlab. org/wiki/index.php/Practical_Primer_on_the_UVa_Target

[69] C. Keith et al., Nucl.Instrum.Meth. A501, 327 (2003)

[70] M. Goldman, Spin Temperature and Nuclear Magnetic Resonance in Solids (Oxford University Press, Oxford, 1970) ISBN 0-19-851251-1

[71] M. Amarian et al., Nuclear Instruments and Methods in Physics Research Section A: Accelerators, Spectrometers, Detectors and Associated Equipment 460, 239 (2001), ISSN 0168-9002

[72] http://ikpe1101.ikp.kfa-juelich.de/cosy-11/exp/drift_chambers/ DriftChambers_E.html, [Online; accessed 22-Sep-2013]

[73] Brooks, W., "CLAS - a large acceptance spectrometer for intermediate energy electromagnetic nuclear physics," in 15th Particles and Nuclei International Conference 1999, Uppsala (SE), 06/10/1999-06/16/1999(1999)

[74] http://www-meg.phys.cmu.edu/ bellis/dc/dcintro.html, [Online; accessed 22-Sep-2013] 
[75] B. Mecking et al., Nuclear Instruments and Methods in Physics Research Section A: Accelerators, Spectrometers, Detectors and Associated Equipment 503, 513 (2003), ISSN 0168-9002

[76] D. C. Doughty Jr., J. Englert, R. Hale and S. Lemon, A VXIbus Based Trigger for the CLAS Detector at CEBAF, Tech. Rep. CLAS-NOTE-91-017 (JLAB, 1991)

[77] “The eg4 wiki," https://clasweb.jlab.org/rungroups/eg4/wiki/index. php/Main_Page, [Online; accessed 22-Sep-2013]

[78] V. Blobel et al., "The BOS system for the CLAS detector: Dynamic Memory Management," (1995)

[79] P. Bosted, Private communications (2010)

[80] P. Bosted, S. Kuhn, Y. Prok , Raster Corrections for EGlb, Tech. Rep. CLAS-Note 2003-008 (JLAB, 2003)

[81] A. Klimenko and S. Kuhn, Momentum Corrections for E6, Tech. Rep. CLAS-Note 2003-005 (JLAB, 2003)

[82] S. E. Kuhn, http://clasweb.jlab.org/rungroups/eg1-dvcs/wiki/index. php, [Online; accessed 16-Jun-2009]

[83] S. Tkachenko, Neutron Structure Functions Measured with Spectator Tagging, Ph.D. thesis, Old Dominion University (December 2009)

[84] P. Bosted and H Avakian, Multiple Scattering and Stray Magnetic Field Corrections for Tracking in the Presence of Target Field, Tech. Rep. CLAS-Note 2006-006 (JLAB, 2006)

[85] W. R. Leo, Techniques for nuclear and particle physics experiments: a how-to approach (Springer, 1994)

[86] M. Osipenko, A. Vlassov and M. Taiuti, A VXIbus Based Trigger for the CLAS Detector at CEBAF, Tech. Rep. CLAS NOTE 2004-020 (JLAB, 2004)

[87] K. Egiyan, Determination of Electron Energy Cut Due to the CLAS EC Threshold, Tech. Rep. CLAS-NOTE-1999-007 (JLAB, 1999)

[88] "The eg4 collaboration," http://clasweb.jlab.org/shift/eg4/, [Online; accessed 22-Sep-2013] 
[89] P. Bosted, Pair-Symmetric and Pion Backgrounds for EG1b, Tech. Rep. CLAS-NOTE-2004-005 (JLAB, 2004)

[90] S. P. Phillips, http://clasweb.jlab.org/rungroups/eg4/wiki/index.php/ October_14\%2C_2011, [Online; accessed 22-Sep-2013]

[91] P. Bosted, NH3 Correction for ND3 Target: EG1-DVCS Technical Note 17, Tech. Rep. (2011) http://wwwold.jlab.org/Hall-B/secure/eg1-dvcs/ technotes/hind/hind.pdf

[92] S. Koirala, EG1-DVCS Part-C Target Contamination, Tech. Rep. (2012)

[93] S. Kuhn and K. Adhikari, "Data Analysis for EG4 - extraction of $g_{1}$ from data," (2013)

[94] K. Abe et al. (E143 Collaboration), Phys. Rev. D 58, 112003 (Oct 1998)

[95] T.V. Kuchto and N.M. Shumeiko, Nuclear Physics B 219, 412 (1983), ISSN 05503213

[96] L. W. Mo and Y. S. Tsai, Rev. Mod. Phys. 41, 205 (Jan 1969)

[97] P. Bosted and M. Christy, Phys.Rev. C77, 065206 (2008), arXiv:0711.0159 [hep-ph]

[98] R. G. Fersch et al., "Precise Determination of Proton Spin Structure Functions at Low to Moderate $Q^{2}$ with CLAS,"' (October 2012), To be published

[99] N. Guler et al., "Precise Determination of Deuteron and Neutron Spin Structure Functions at Low to Moderate $Q^{2}$ with CLAS," (October 2013), To be published

[100] M. Holtrop, “GSIM: CLAS GEANT Simulation,” http://nuclear.unh.edu/ -maurik/gsim_info.shtm1, [Online; accessed 22-Sep-2013]

[101] B. Dey, Differential cross section and polarization extractions for $\gamma p \rightarrow K^{+} \Sigma^{0}$ and $\gamma p \rightarrow \phi p$ using CLAS at Jefferson Lab, towards a partial wave analysis in search of missing baryon resonances, Ph.D. thesis, Carnegie Mellon University (July 2011)

[102] J. Zhang, Exclusive $\pi^{-}$Electro-production from the Neutron in the Resonance Region, Ph.D. thesis, Old Dominion University (May 2010) 


\section{APPENDIX A}

\section{DERIVATION OF THE GDH SUM RULE}

The real photon Gerasimov-Drell-Hearn (GDH) sum rule is derived [25, 26] using the general assumptions of Lorentz and Gauge invariance (in the form of low energy theorem), unitarity (in the form of optical theorem) and causality (in the form of an unsubtracted dispersion relation for the forward Compton scattering, also assuming crossing symmetry) [32]. For the forward Compton scattering of a real photon on a nucleon, the scattering amplitude $T(v, \theta=0)$ is given as follows in terms of the spin-independent and spin dependent amplitudes $f(v)$ and $g(v)$ :

$$
T(v)=\overrightarrow{\varepsilon^{*}} \cdot \vec{\varepsilon} f(v)+i \vec{\sigma} \cdot\left(\overrightarrow{\varepsilon^{*}} \times \vec{\varepsilon}\right) g(v)
$$

where $\vec{\varepsilon}$ and $\vec{\varepsilon}^{*}$ are the polarization vectors of the incident and scattered photons respectively. In order for the crossing symmetry to hold true, the T-matrix must be symmetric under the exchange of the incoming and the outgoing photons, $\overrightarrow{\varepsilon^{*}} \leftrightarrow \vec{\varepsilon}$ and $v \rightarrow-v$, implying that the amplitudes $f$ and $g$ are an even and odd functions of $v$ respectively. These amplitudes can be separately determined by scattering circularly polarized photons off a longitudinally polarized nucleon, with $f$ and $g$ obtained from the cases of parallel or anti-parallel target polarization with respect to the photon momentum $\vec{q}$. The polarization vectors for a left-handed $(+1)$ and right-handed $(-1)$ circularly polarized photons moving along $\mathrm{z}$-axis are given by:

$$
\varepsilon_{ \pm}= \pm \frac{1}{\sqrt{2}}\left(\hat{e_{x}} \pm i \hat{e_{y}}\right)
$$

with the transverse gauge $(\varepsilon \cdot q=0)$ used and photon 4-momentum and polarization defined as $q=(v, \vec{q})$ and $\varepsilon=(0, \vec{\varepsilon}$ with the condition $q \cdot q$.

Unitarity of scattering matrix means that the imaginary parts of the forward amplitudes f and $g$ are connected to the total photoabsorption cross sections via the optical theorem as follows:

$$
\operatorname{Im} f(v)=\frac{v}{8 \pi}\left(\sigma_{\frac{1}{2}}(v)+\sigma_{\frac{3}{2}(v)}\right)=\frac{v}{4 \pi} \sigma_{T}
$$

and

$$
\operatorname{Im} g(v)=\frac{v}{8 \pi}\left(\sigma_{\frac{1}{2}}(v)-\sigma_{\frac{3}{2}(v)}\right)=\frac{v}{4 \pi} \sigma_{T T}
$$


with the helicity dependent cross-sections as defined earlier in section.

At small photon energies, the amplitudes can be expanded in powers of $v$ with the low energy theorem (LET) resulting in

$$
\begin{gathered}
f(v)=\frac{Z^{2} e^{2}}{4 \pi M}+(\alpha+\beta) v^{2}+\mathscr{O}\left(v^{4}\right) \\
g(v)=\frac{\kappa^{2} e^{2}}{8 \pi M^{2}} v+\gamma_{0} v^{3}+\mathscr{O}\left(v^{5}\right)
\end{gathered}
$$

where $\mathrm{Z}$ is the charge of the target (in units of "e"). In the expansion for the spinindependent amplitude $f(v)$, the leading term $\mathrm{f}(0)$ is the classical Thomson scattering result, the $O\left(v^{2}\right.$ term describes Raleigh scattering in terms of the electric and magnetic dipole polarizabilities $\alpha$ and $\beta$ respectively. On the other hand, in the expansion of the spin-flip amplitude $g$, the leading term is associated with he anomalous magnetic moment $(\kappa)$, and the next $O\left(v^{3}\right)$ term is related to the forward spin polarizability $\gamma_{0}$, which carries the information on the spin structure.

Finally, the dispersion relations for the two forward amplitudes $f(v)$ and $g(v)$ are derived using the analytic properties of the forward Compton scattering amplitudes with unitarity and crossing symmetry. For the spin-averaged amplitude $f(v)$, the KramersKronig relation from optics, which connects the real part of $f$ with an integral over the imaginary part of $\mathrm{f}$ :

$$
\operatorname{Re} f(v)=\frac{2}{\pi} \mathscr{P} \int_{v_{0}}^{\infty} d v^{\prime} v^{\prime} \frac{\operatorname{Im} f\left(v^{\prime}\right)}{v^{\prime 2}-v^{2}}
$$

Where $\mathscr{P}$ denotes the principal value of the integral. The imaginary part is next replaced by the total cross-section using the optical theorem, so the dispersion relation becomes:

$$
\operatorname{Re} f(v)=f(0)+\frac{v^{2}}{2 \pi^{2}} \mathscr{P} \int_{v_{0}}^{\infty} d v^{\prime} \frac{\sigma_{T}\left(v^{\prime}\right)}{v^{\prime 2}-v^{2}}
$$

with $f(0)$ being the Thomson limit of eq. 159. Because the total cross section rises in a slow logarithmic manner above the resonance region, a subtraction is made at $v=0$ to ensure the integral converges.

Applying through the same method, an unsubtracted dispersion relation is derived for the spin-dependent amplitude as follows:

$$
\operatorname{Reg}(v)=\frac{v}{4 \pi^{2}} \mathscr{P} \int_{v_{0}}^{\infty} d v^{\prime} v^{\prime} \frac{\sigma_{\frac{1}{2}}\left(v^{\prime}\right)-\sigma_{\frac{3}{2}}\left(v^{\prime}\right)}{v^{\prime 2}-v^{2}}
$$


where, now the optical theorem is used to replace the imaginary part of the amplitude with the helicity dependent cross-section difference. In this spin-dependent case, the nonsubtraction hypothesis is used because unlike the total cross-section the helicity dependent cross-section difference does not rise at large $v^{\prime}$, but decreases fast enough to ensure the convergence of the integral without any subtraction.

Finally, by comparing the first order i.e. $O(v)$ terms in Eq.160 and Eq.163, we arrive at the GDH sum rule as follows:

$$
I \equiv-\frac{\alpha}{2 M^{2}} \kappa^{2}=\frac{1}{4 \pi^{2}} \int_{v_{0}}^{\infty} \frac{d v}{v}\left[\sigma_{\frac{1}{2}}(v)-\sigma_{\frac{3}{2}}\right]
$$

where $\alpha=\frac{e^{2}}{4 \pi}$. One can similarly derive the sum rules for the electric and magnetic polarizabilities and the forward spin polarizability. 


\section{APPENDIX B}

\section{FFREAD CARDS USED BY GSIM}

TABLE 2. Some of the ffread cards \& their values which are used as GSIM input parameters.

\begin{tabular}{|l|l|}
\hline Cards & Values \\
\hline MAGTYPE & 2 \\
\hline MAGSCALE & -0.58290 .0 (for 1.337 GeV) \\
\hline MAGSCALE & -0.38860 .0 (for 1.993 GeV) \\
\hline GEOM & 'ALL' \\
\hline NOMC & 'EC' 'SC' 'CC' 'DC' \\
\hline NOGEOM & 'MINI' 'ST' 'TG2' 'TG' 'SOL' \\
\hline NOGEOM & 'PTG' 'FOIL' \\
\hline NOMATE & 'PTG' 'FOIL' \\
\hline PTGIFIELD & 1 \\
\hline TMGIFIELD & 1 \\
\hline TMGIFIELDM & 1 \\
\hline TMGFIELDM & 51.0 \\
\hline TMGSCALE & 0.979 \\
\hline PTGMAXRAD & 300.0 \\
\hline MGPOS & $0.00 .0-100.93$ \\
\hline BAFF & 3.9 .165 .39 .180 .59 .195 .8 \\
\hline RUNG & 50556 \\
\hline AUTO & 1 \\
\hline KINE & 1 \\
\hline
\end{tabular}




\section{VITA}

Krishna P. Adhikari

Department of Physics

Old Dominion University

Norfolk, VA 23529

Krishna Adhikari is from Vijayanagar, Chitwan, Nepal. He received his Bachelor of Science (with courses in Physics, Chemistry and Mathematics) in 1997 from Birendra Multiple Campus (Tribhuvan University), in Bharatpur, Nepal. After that he taught science and mathematics courses for a year in Naya Kiran Secondary School, in Bharatpur, Chitwan, Nepal. In 1998, he joined Masters of Science program in Physics in Tribhuvan University, Kathmandu Nepal completing and graduated in 2000. Then, from 2001 to 2004, he taught undergraduate physics courses as part time instructor in four different colleges in Kathmandu, Nepal (Amrit Science College, Patan Multiple Campus, Asian College of Higher Studies, and BernHardt College). In 2004, he joined Old Dominion University for the graduate program in physics and received M.S. degree in 2006 . Then he joined experimental Nuclear Physics group at the Old Dominion University in 2007 to work on the subject of this dissertation under the supervision of Dr. Sebastian E. Kuhn. He defended the Ph.D. dissertation on 14 November 2013.

Typeset using $\mathrm{LTT}_{\mathrm{E}} \mathrm{X}$. 